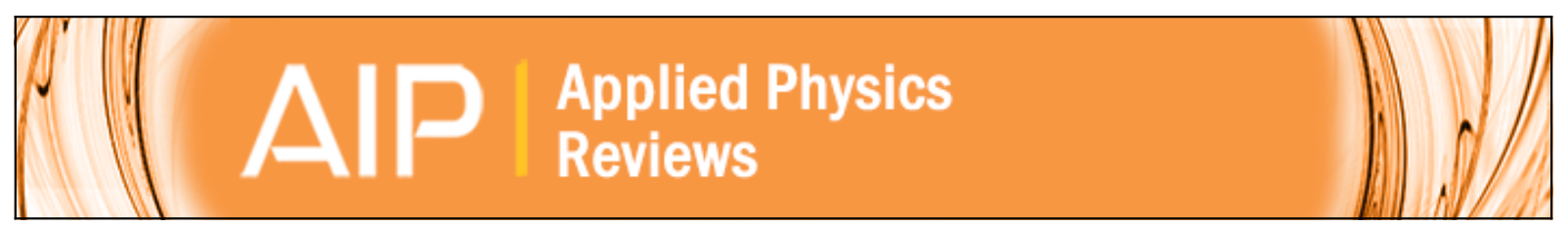

\title{
Heterogeneous 2.5D integration on through silicon interposer
}

Xiaowu Zhang, Jong Kai Lin, Sunil Wickramanayaka, Songbai Zhang, Roshan Weerasekera, Rahul Dutta, Ka Fai Chang, King-Jien Chui, Hong Yu Li, David Soon Wee Ho, Liang Ding, Guruprasad Katti, Suryanarayana Bhattacharya, and Dim-Lee Kwong

Citation: Applied Physics Reviews 2, 021308 (2015); doi: 10.1063/1.4921463

View online: http://dx.doi.org/10.1063/1.4921463

View Table of Contents: http://scitation.aip.org/content/aip/journal/apr2/2/2?ver=pdfcov

Published by the AIP Publishing

\section{Articles you may be interested in}

Through-silicon via-induced strain distribution in silicon interposer

Appl. Phys. Lett. 106, 141905 (2015); 10.1063/1.4915604

Plasticity mechanism for copper extrusion in through-silicon vias for three-dimensional interconnects

Appl. Phys. Lett. 103, 211906 (2013); 10.1063/1.4833020

Low-temperature Al-Ge bonding for 3D integration

J. Vac. Sci. Technol. B 30, 06FK01 (2012); 10.1116/1.4762844

Characterization of thermal stresses in through-silicon vias for three-dimensional interconnects by bending beam technique

Appl. Phys. Lett. 100, 041901 (2012); 10.1063/1.3678020

Process integration for through-silicon vias

J. Vac. Sci. Technol. A 23, 824 (2005); 10.1116/1.1864012

\section{AlP $\left.\right|_{\text {Applied Physics }} ^{\text {Journal of }}$}

Journal of Applied Physics is pleased to announce André Anders as its new Editor-in-Chief 


\title{
Heterogeneous 2.5D integration on through silicon interposer
}

\author{
Xiaowu Zhang, Jong Kai Lin, Sunil Wickramanayaka, Songbai Zhang, \\ Roshan Weerasekera, Rahul Dutta, Ka Fai Chang, King-Jien Chui, Hong Yu Li, \\ David Soon Wee Ho, Liang Ding, ${ }^{\text {a) }}$ Guruprasad Katti, Suryanarayana Bhattacharya, ${ }^{\text {b) }}$ \\ and Dim-Lee Kwong \\ Institute of Microelectronics, A*STAR (Agency for Science, Technology and Research), 11 Science Park Road, \\ Singapore Science Park II, Singapore 117685
}

(Received 14 September 2014; accepted 18 March 2015; published online 23 June 2015)

\begin{abstract}
Driven by the need to reduce the power consumption of mobile devices, and servers/data centers, and yet continue to deliver improved performance and experience by the end consumer of digital data, the semiconductor industry is looking for new technologies for manufacturing integrated circuits (ICs). In this quest, power consumed in transferring data over copper interconnects is a sizeable portion that needs to be addressed now and continuing over the next few decades. 2.5D Through-Si-Interposer (TSI) is a strong candidate to deliver improved performance while consuming lower power than in previous generations of servers/data centers and mobile devices. These low-power/high-performance advantages are realized through achievement of high interconnect densities on the TSI (higher than ever seen on Printed Circuit Boards (PCBs) or organic substrates), and enabling heterogeneous integration on the TSI platform where individual ICs are assembled at close proximity ( $<1 \mathrm{~mm}$ separation) compared with several centimeters on a typical PCB. In this paper, we have outlined the benefits of adopting 2.5D TSI technology and also highlighted the current day approaches to implement this technology in Si fabrication facilities, and in assembly/packaging factories. While the systems and devices that power the mobile society benefit from exploiting advantages of 2.5D integration on TSI, there do exist surmountable challenges that need to be addressed for this relatively new technology to be used in high volume production of next generation semiconductor devices. The key areas of focus and challenges include: Technology planning and design-execution that are necessary for harnessing 2.5D TSI for building systems, processing flow for the fabrication of $100 \mu \mathrm{m}$ thick TSI at acceptable costs, manufacturing flow for assembling multiple ICs on a $100 \mu \mathrm{m}$ thick TSI in a repeatable, and reliable manner, thermo-mechanical analysis and optimization for addressing warpage issues, and thermal management for addressing heat dissipation. We have outlined design, manufacturing methodologies, and challenges, along with solutions to the challenges associated with taking 2.5D TSI technology to high volume production within the next few years. () 2015 AIP Publishing LLC.
\end{abstract}

[http://dx.doi.org/10.1063/1.4921463]

\section{TABLE OF CONTENTS}

I. SYSTEMS TRENDS AND 2.5D/3D TECHNOLOGY INTEGRATION BENEFITS .... 2

A. Interconnect bottleneck .............. 3

1. More-on-Moore SoC scaling .......... 3

2. More-than-Moore system scaling ...... 3

3. Application examples ............. 5

B. General summary in advancement ....... 6

C. Challenges for heterogeneous $2.5 \mathrm{D}$ integration

on TSI.........................

\footnotetext{
a) Present address: Inphi International Pte. Ltd., 988 Toa Payoh North, \#05$01 \&$ \&05-07/08, Singapore 319002.

b)Electronic mail: bhattass@ime.a-star.edu.sg
}

1. Technology planning and designexecution..................... 7

2. Processing flow for fabrication of $100 \mu \mathrm{m}$ thick TSI................ 7

3. Manufacturing flow for assembling multiple ICs on a $100 \mu \mathrm{m}$ thick TSI ... 7

4. Thermo-mechanical analysis and optimization ....................

5. Thermal management of 2.5D TSI.....

6. Development of next generation manufacturing equipment............

II. KEY TECHNOLOGY MODULES FOR 2.5D HETEROGENEOUS INTEGRATION ......... 8

A. TSV module.................... 8

1. TSV deep Si etch process ........... 9

2. TSV liner deposition.............. 10

3. Seed layer and barrier layer deposition .. 11 


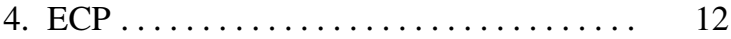

5. CMP................... 13

B. BEOL and RDL scheme ........... 13

C. Bumping ................. 15

1. Solder bumping and $\mathrm{Cu}$ pillar bumps.... 15

2. Bumps in 2.5D TSI package ........ 15

3. Fabrication of $\mathrm{Cu}$ pillar micro-bumps ... 15

4. Reliability assessment of $\mathrm{Cu}$ pillar bumps $\ldots \ldots \ldots \ldots \ldots \ldots \ldots \ldots$

D. Thin wafer handling $\ldots \ldots \ldots \ldots \ldots \ldots \ldots$

1. Carrier substrates ...............

2. Temporary bonding and debonding .....

3. Adhesives requirement .............

III. 2.5D TSI ASSEMBLY CHALLENGES . . . . .

A. Introduction $\ldots \ldots \ldots \ldots \ldots \ldots \ldots$

B. TSI package assembly process flow....... 1. Chip-on-substrate (CoS) first assembly

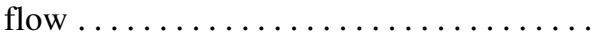

2. Chip-on-chip (CoC) first assembly flow .

3. Chip-on-wafer (CoW) first assembly flow ................... 22

C. Assembly Challenges and Warpage Control. 24

D. Summary ........................ 24

IV. 2.5D TSI TEST AND CHARACTERIZATION . 25

A. Test structures for 2.5D TSI electrical

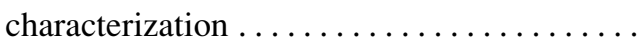

1. TSV characterization structures........

2. High density wiring characterization ....

3. Chip-to-chip link characterization......

4. Micro joint reliability characterization...

5. Decoupling capacitors.............

V. THERMAL CONSIDERATIONS OF 2.5D

PACKAGES

A. Thermal challenges in the context of $2.5 \mathrm{D}$

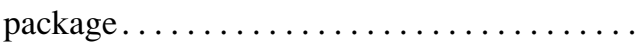

B. Passive cooling $\ldots \ldots \ldots \ldots \ldots \ldots \ldots \ldots$

1. TSV thermal enhancement..........

2. Effect of TSV interposer on package thermal performance .............

3. Thermal characterization of stacked

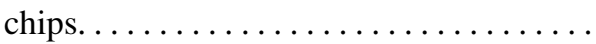

4. Thermal characterization of $2.5 \mathrm{D}$

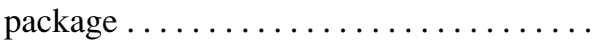

C. Active cooling ..................

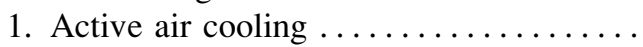

2. Liquid cooling .................

D. Thermal aware $2.5 \mathrm{D}$ design and

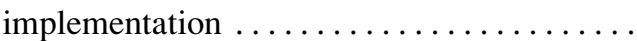

VI. THERMO-MECHANICAL DESIGN AND MODELING FOR 2.5D PACKAGING .......

A. Manufacturability and functionality of TSI .

1. Design rules for avoiding wafer cracking

2. Design rules for reducing copper

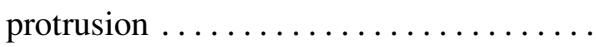

3. Design for reducing mobility change of MOSFETs near TSVs..............

B. Handling of TSV wafers and chips during fabrication and assembling ...........
1. Design for reducing TSV wafer warpage

2. Design for avoiding die-cracking during

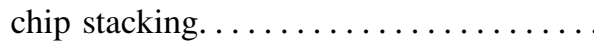

C. Packaging and long-term reliability .......

1. CPI and micro solder bump reliability of TSI ......................

2. Design for enhancing solder joint reliability

of packages with Si carrier ..........

42

43

3. Demonstration a low stress bond pad design for low temperature micro bumps

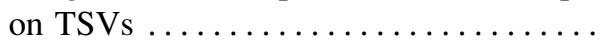

VII. EDA CONSIDERATIONS AND READINESS .

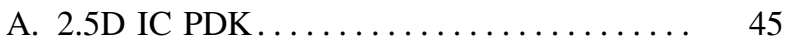

1. Technology LEF files ............. 45

2. PEX rule decks and TSV extraction.... 45

3. DRC/LVS rule decks ........... 46

B. $2.5 \mathrm{D}$ IC EDA flow $\ldots \ldots \ldots \ldots \ldots \ldots \ldots . \ldots \ldots$

1. Design import.............. 46

2. Floor planning and placement ....... 47

3. Routing on $2.5 \mathrm{D}$ TSI ........... 47

4. Parasitic extraction ............. 47

5. SI and PI verification ........... 47

6. DRC and LVS verification......... 47

C. 2.5D functional vehicle design-A case

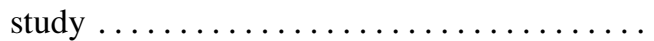

VIII. REVISITING APPLICATIONS AND BENEFITS OF 2.5D TSI TECHNOLOGY ... . 48

A. 2.5D TSI technology-Application space... 48

B. Application roadmap.............. 50

IX. FUTURE OUTLOOK/CONCLUSIONS ...... 50

A. 2.5D TSI technology roadmap ......... 50

1. Solder technology .............. 51

2. Metal-metal bonding ............ 52

B. $2.5 \mathrm{D} / 3 \mathrm{D}$ EDA outlook ............. 53

1. Inability of existing 3D EDA tools to analyze unified 3D netlist .......... 53

2. Employing calibrated TSV lumped "RC"

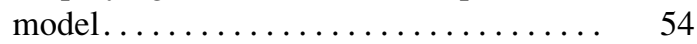

X. SUMMARY $\ldots \ldots \ldots \ldots \ldots \ldots \ldots \ldots \ldots \ldots$

\section{SYSTEMS TRENDS AND 2.5D/3D TECHNOLOGY INTEGRATION BENEFITS}

We live in a mobile society where mobile devices allow us to communicate readily with people that are socially and professionally connected to us. Today's mobile society leverages on the performance and reliability of mobile devices (phones and tablets) and the vast internet infrastructure. In these two areas, amazing technical innovations are strongly driven by the large market size and customer base. Continued innovations are needed as the adoption of mobile devices rapidly penetrates developed as well as emerging economies of the world. Innovations are necessary since such adoption will drive the processing and transfer of large volumes of data in the mobile society.

Recent data show that there is a greater than 10 times increment in the mobile data traffic from 2012 to 2017 and 
the daily data traffic amounts to be quintillion bytes ("Big Data"). ${ }^{1}$ These data originate from all around the world in the forms of climate information, pictures and videos, banking transactions, and global positioning system (GPS) data, etc. Handling such data traffic is dependent on the use of various $\mathrm{Si}$ technologies (e.g., Logic-CMOS, dynamic random access memory (DRAM), BiCMOS, Si Photonics, etc.). A key concern is the performance and power consumption of integrated circuits (ICs) and devices for transferring large amounts of data. In this review paper, we look into the opportunities and trends in the heterogeneous integration of various $\mathrm{Si}$ technologies using 2.5D Through-Si-Interposer (TSI) as a next generation Si technology platform to manufacture next generation IC and devices that will support the explosion in the data traffic.

\section{A. Interconnect bottleneck}

When one looks closely at data traffic at the system/subsystem level (e.g., mobile phones, tablets, data centers, and line cards), the data traffic most typically occurs between the logic and memory devices and is transmitted across $\mathrm{Cu}$ interconnects. With large amounts of data processed or transferred, a key bottleneck is the movement of the data between a logic device and a memory or between 2 or more logic devices. Such data transfer happens over interconnects. Interconnects within computers, mobile devices, and within data-centers are typically using $\mathrm{Cu}$ lines on Printed Circuit Boards (PCBs) or Organic Substrates, or on System-on-Chip (SoC) interconnects-for electrical data transfer. A simplest case that can be considered is that of a multi-core processor transferring data to a nearby memory (known as logicmemory interface) over $\mathrm{Cu}$ interconnects. Such data transfer occurs all the time in a mobile device or in a data-center or in a switching network. It turns out, the performance of, and the power consumed by the logic-memory interface is a critical bottleneck that needs to be addressed for both mobile devices and data-centers that form a central part of today's internet infrastructure.

\section{More-on-Moore SoC scaling}

The challenge with improving the efficiency and speed of the logic-to-memory interface is also referred to as the "memory-wall." This is because logic-device (or processor) performance is steadily improving over past decades due to CMOS technology advancement that is guided by Moore's law. $^{2}$ The More-on-Moore miniaturization ${ }^{3}$ drives the minimum chip feature size (transistor gate length) to shrink about $50 \%$ every 18 months, allowing for faster transistors on logic devices (processors). Processor optimization has also led to the use of multi-core (8-core, 32-core, 64-core, and so on) processors to enhance the performance of advanced processors called Central Processing Units (CPU). In a high performance system, this means that each of these individual processor-cores needs to have separate access channel to data in the memory. However, there is a limitation to how fast multiple processors can exchange data with increasingly large amounts of memory. This is a fundamental interconnect related challenge faced by today's PCBs and electronic packages. Therefore, they cannot support the density of interconnects that is needed to support this data transfer. In addition to interconnect density, the length of the interconnects on PCBs is quite long (order of centimeters), which means that significantly higher power consumption is required when multiple processors send and receive data from memory devices.

CMOS technology miniaturization (Moore's law ${ }^{2}$ ) can be translated into system level performance improvements by using the System-On-Chip architecture-where both logic and memory are fabricated in the same $\mathrm{Si}$ wafer. However, as mentioned above, the advent of multi-core processors that need to have large amounts of adjacent memory (several Gigabytes) places several limitations on the Moore's law-enabled SoC architecture in terms of the sheer size of $\mathrm{Si}$ needed (larger Si chip means lower yields). In addition, the high performance Si technology for producing logic processors is highly unsuitable for making low-power memory functions (e.g., DRAM). The separation of the logic and memory functions into discrete packages places the burden on the PCB, and the electronic packages. When the complexity of ICs increases, the number of I/O pins rises exponentially according to the well known Rents rule, ${ }^{4}$ thereby giving rise to increased wiring demands ${ }^{5,6}$ on the system level. For next generation systems, the PCB and electronic packages face serious challenges from (a) limited interconnect density of electronic packages that connect logic and memory, (b) I/O circuit power consumption related to higher parasitics from longer $\mathrm{PCB}$ and package wire lengths, and (c) power disadvantage $\left(1 / 2\right.$ f.C. $\left.V^{2}\right)$ at higher clock rates are typically used to transfer large amounts of data within systems with I/O count limitations. Package (C4) bump-pitch has scaled at a significantly slower rate in comparison with CMOS technology nodes, and it demonstrates the manifestation of the interconnect challenge. ${ }^{7}$

Therefore, traditional electronic packaging combined with Moore's Law presents a bottleneck for system scaling, i.e., increasing system speed, reducing system power, and shrinking system size.

\section{More-than-Moore system scaling}

In order to provide a multi-generational solution to address the challenges to system-scaling, one needs an alternative approach that overcomes the limitations on system scaling placed by the memory-wall, limitations of Moore's law/SoC architecture, and the lack of interconnect scaling on PCBs and electronic packages. First, high performance systems need optimized Si technologies to obtain the necessary performance and power requirements of different parts of the system, namely, logic gates, memory cells, RF/Analog blocks, etc. Thus, one needs to think in terms of "disintegrating" the SoC to achieve optimization of given function: (a) Logic-speed and power, (b) memory density/ power/ performance, (c) RF/Analog performance, and (d) optimized interconnect that links these features to make high performance/low-power systems. With the ability to optimize $\mathrm{Si}$ technologies based on the functions, one can develop both memory and logic (CPU) ICs with the necessary number of I/O counts that can overcome the memory-wall. To 
interconnect the tens to hundreds of thousands of I/Os, between logic ICs and memory ICs, one can use fine pitch copper wiring that can be readily derived from CMOS copper interconnects using Si fabrication. This provides Si substrate to connect several thousands of interconnects between ICs, and deliver power and ground supply to the ICs on the Si substrate. Second, unpackaged individual ICs are attached using micro-solder-joints or micro-bumps to the Si substrate containing fine pitch interconnects (pitch capable of reaching $<2 \mu \mathrm{m}$ line/space compared to $10-20 \mu \mathrm{m}$ line/space on organic packages and PCBs). Such unpackaged ICs have much reduced package parasitics. The signal, power, and ground connections are provided by Through-Si-Vias (TSVs), ${ }^{8}$ enabling electrical connections from the bottom side of the $\mathrm{Si}$ substrate to the front side where the ICs are attached. Such a Si substrate is called a TSI. ${ }^{9-11}$ This technology referred to as $2.5 \mathrm{D}$ integration (or 2.5D ICs) using TSIs offers significant benefits to overcome limitations to system-scaling and is considered a game-changer for current and future systems that are expected to drive mobile-handheld and data-center applications for decades to come. The term 2.5D stems from the fact that using TSI technology for stacking ICs side-byside on a TSI can be considered a midpoint between traditional ICs in package representing 2D integration, and chips stacked on top of one another that represents 3D integration. The TSI offers a technology platform to integrate disparate technologies (such as CMOS, memory, sensors, high density copper interconnects, optical interconnects supported by Si photonics, etc.) and thus enable heterogeneous integration. This form of integration is also known as Morethan-Moore integration.

Figure 1(a) shows an example of a TSI platform which provides the necessary high density interconnects to address the gap between chip scaling and system wiring in many of the heterogeneous systems. TSI allows designers to connect multiple ICs with high density and significantly shorter $(<1 \mathrm{~mm})$, fine pitch $(<4 \mu \mathrm{m})$ sub micron interconnects (than provided by traditional packaging), and microbump (pitch $<40 \mu \mathrm{m}$ ) technology. TSVs provide the access to a package/PCB for power supply or ground and external I/Os.

Figure 1(b) illustrates how the fine pitch (pitch $=40 \mu \mathrm{m})$ micro-bumps connect field-programmable gate array (FPGA) slice to the TSV interposer. The C4 bumps (pitch $=200 \mu \mathrm{m}$ ) in turn connect the TSI to the package. Large package balls (pitch $=1000 \mu \mathrm{m}$ ) connect the package to the PCB.

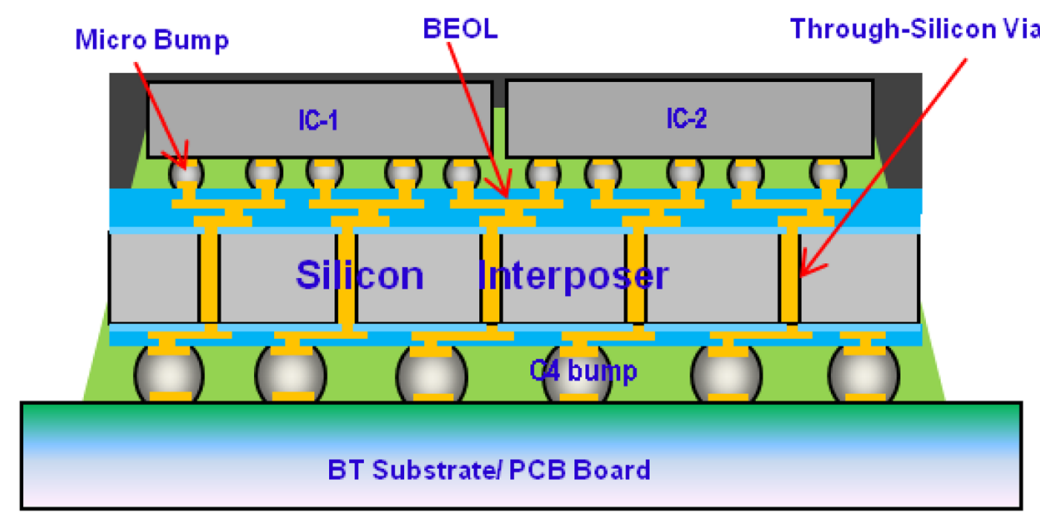

(a)

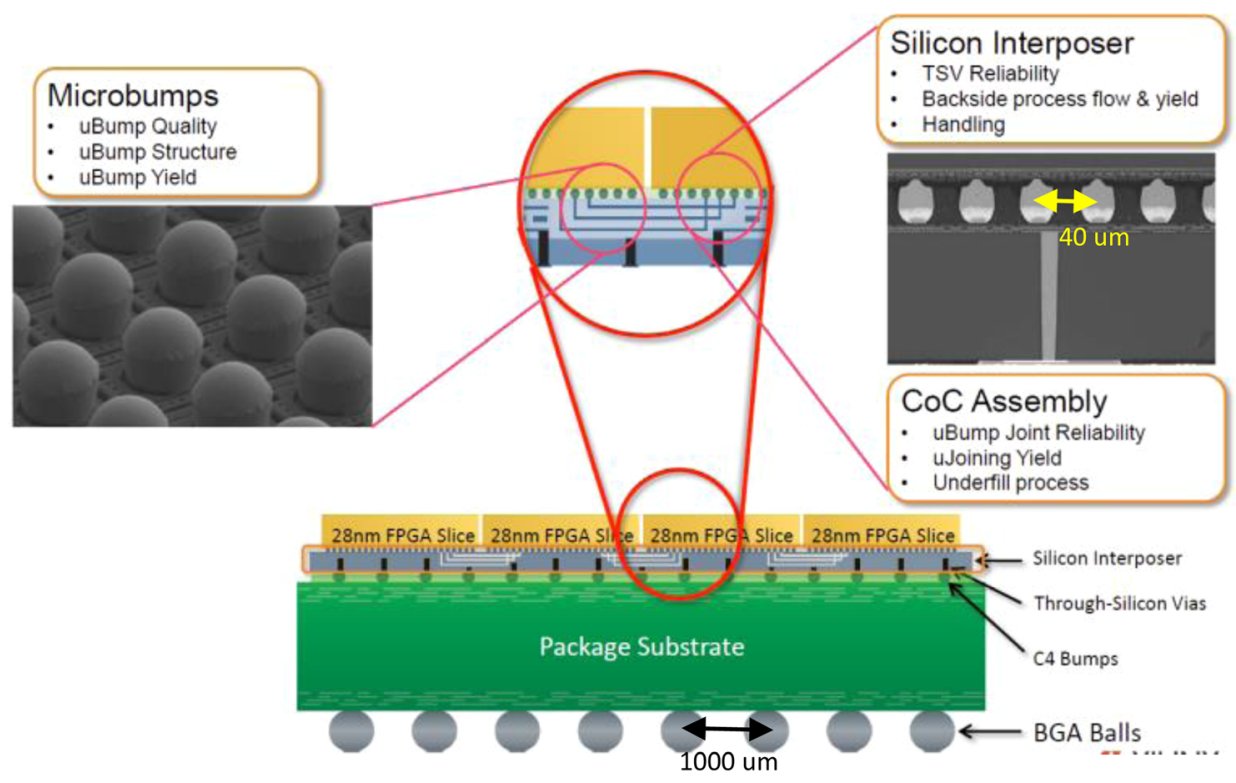

FIG. 1. (a) Schematic of IC integration on a TSI platform. (b) Cross-section of the Xilinx 2.5D TSI based Virtex-7HT FPGA. ${ }^{12}$ Reproduced with permission from Madden et al., "Advancing high performance heterogeneous integration through die stacking," in Proceedings of European Solid-State Device Research Conference (ESSDERC) (2012). Copyright 2012 Institute of Electrical and Electronics Engineers.

(b) 


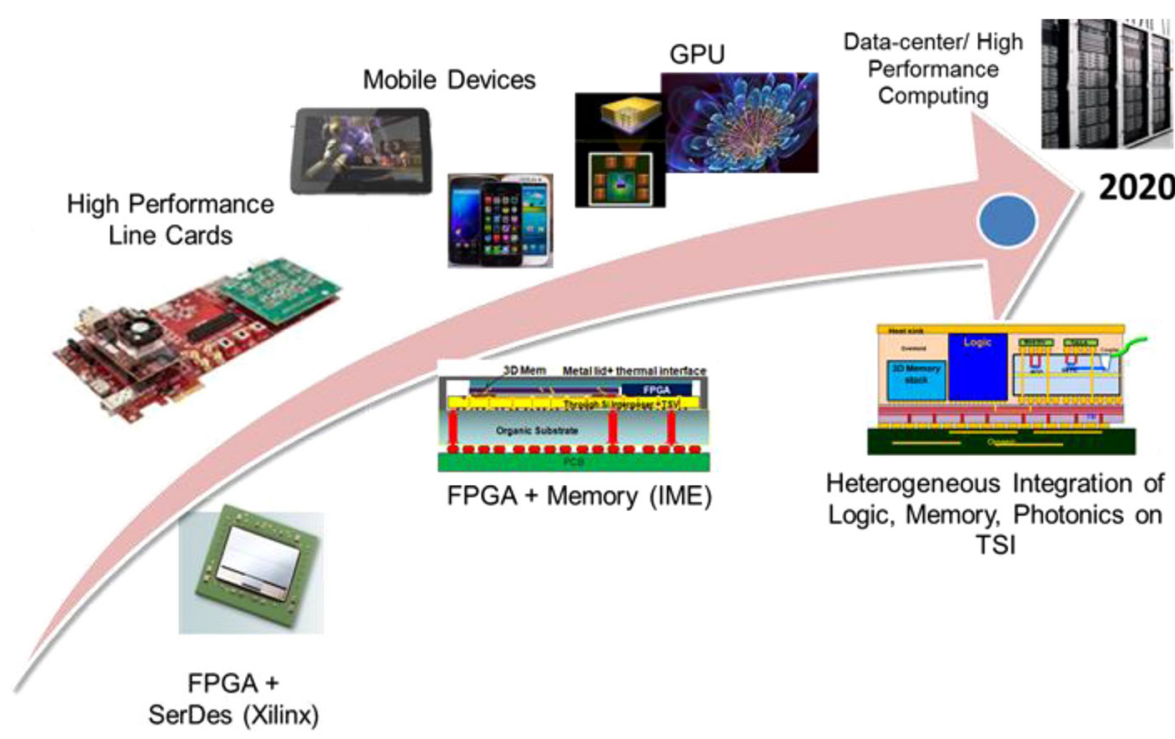

FIG. 2. Si interposer application examples and development roadmap.
Over the past few years, a significant research effort has been invested on 2.5D integration technologies encompassing fabrication, assembly, packaging, and design automation tools and electrical design issues such as signal integrity/ power integrity $(\mathrm{SI} / \mathrm{PI}){ }^{13}$ This review paper attempts to capture the advantages of this technology and reviews major challenges and solutions developed by the industry, IME, and other research institutes.

\section{Application examples}

Figure 2 shows a proposed roadmap for the application of 2.5D TSI technology for mobile devices and data centers. In 2012, Xilinx ${ }^{12}$ demonstrated virtex-7 FPGA with 2.5D TSI technology integrating high density gate arrays with high-speed Serializer-Deserializer (SERDES). The next phase of heterogeneous integration using 2.5D TSI will allow the integration of high-speed Graphic Processor Units (GPU) or Application Processor Engines (APE) with high density 3D stacked memories (High-Band-Width Memory $(\mathrm{HBM})^{14}$ and Hybrid Memory Cube (HMC) ${ }^{15}$ ) for graphic computing, and mobile tablets. As data traffic continues to explode, optical interconnects are needed for transferring large volumes of data between racks in data centers and high performance computers. In such applications, the photonic IC (PIC) ${ }^{16}$ needs to be in close proximity with logic device/ SERDES. 2.5D TSI can heterogeneously integrate logic and memory subsystems with photonics that drive optical data communications at sub $\mathrm{pJ} / \mathrm{bit}$ energy efficiencies. Thus $2.5 \mathrm{D}$ TSI technology has the potential to be a key enabler in high speed data communications in the next several decades.

Figure 3 shows a larger picture of the Xilinx Virtex 7HT FPGA. The individual FPGA slices are manufactured using an optimized $28 \mathrm{~nm}$ CMOS technology, while the SERDES is manufactured using $28 \mathrm{~nm}$ high-performance CMOS technology. This optimized technology choice for the FPGA and SERDES functions allows unprecedented $2.8 \mathrm{~Tb} / \mathrm{s}$ data transfer capability using 2.5D ICs at lower power consumption. Figure 5 shows how 2.5D/3D TSV/TSI technology can help reduce the footprint of a state-of-the-art logic processor
(CPU) and DDR4 DRAM system. On the left side, we show a PCB which is typically greater than $20 \mathrm{~cm}$ on one side. On either sides of the packaged CPU, there are multiple banks of packaged DDR4 memories. The length of interconnect between CPU and memory IOs can be of the order of $10 \mathrm{~cm}$. This results in higher parasitic capacitances, as well as signal reflections due to different physical boundaries. In addition, due to the required bandwidth (BW) of $4 \mathrm{~Tb} / \mathrm{s}$ between CPU and DRAM total power can be up to $640 \mathrm{~W}(20 \mathrm{pJ} / \mathrm{bit}$ energy consumption for IOs). On the right side of Figure 4, the same system is implemented on a $2.5 \mathrm{D}$ TSI that is around $40 \mathrm{~mm} \times 40 \mathrm{~mm}$ in size. The four banks of DDR4 are replaced by four 3D stacked high density DRAMs that are in bare die form. The 3D DRAMs are attached to the TSI (yellow color) with fine pitch micro-bumps that can connect thousands of 3D DRAM IOs to the TSI. The CPU, also in an unpackaged, bare die form, is connected to the TSI through fine pitch micro-bumps. The thousands of IOs between the

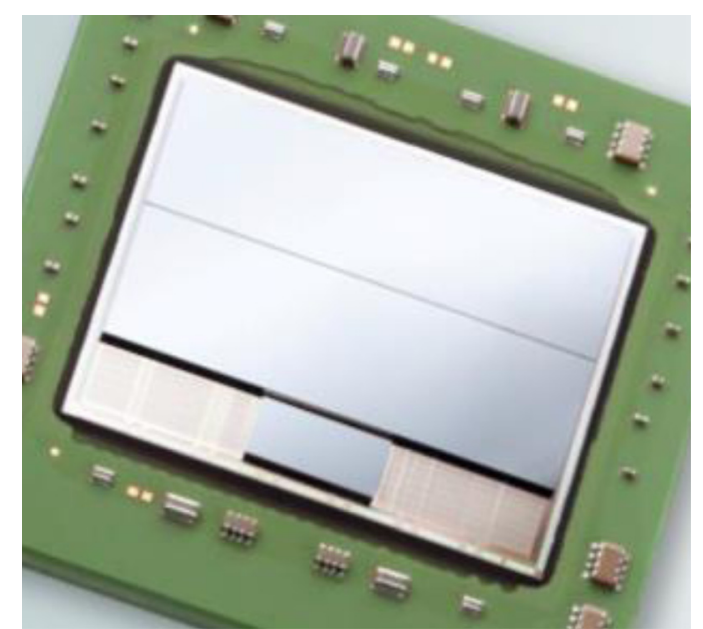

FIG. 3. Xilinx Virtex 7HT with a 2 slices of FPGA integrated with SERDES IC. ${ }^{12}$ Reproduced with permission from Madden et al., "Advancing high performance heterogeneous integration through die stacking," in Proceedings of European Solid-State Device Research Conference (ESSDERC) (2012). Copyright 2012 Institute of Electrical and Electronics Engineers. 


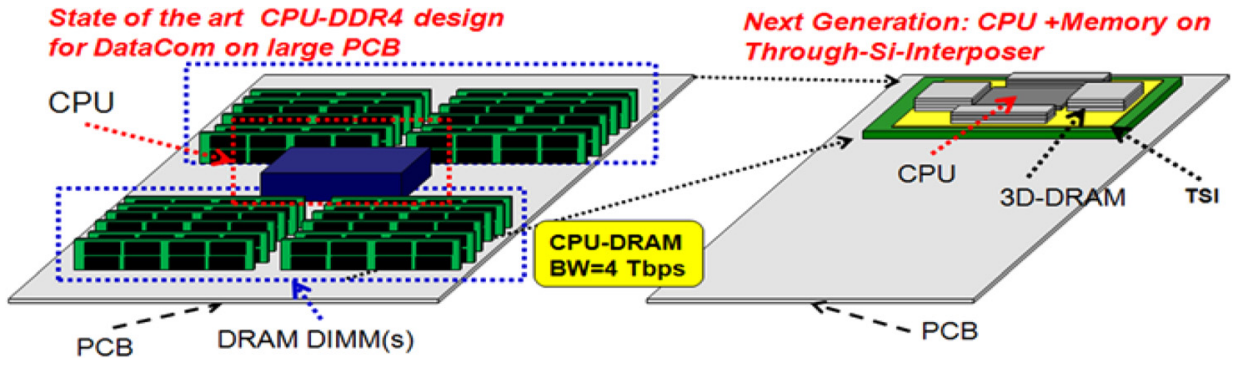

FIG. 4. A typical CPU-memory system showing (a) higher interconnect density and (b) shorter interconnects in TSI based system.

CPU and 3D DRAM are laid out on the TSI using fine pitch copper interconnects. Typically, interconnects in the TSI can support up to 1000 connections per $\mathrm{mm}$ cross-section. Because we are using bare die that can be assembled at close proximity $(<1 \mathrm{~mm})$, the length of interconnect can be around $10 \mathrm{~mm}$, leading to a significantly lower energy consumption of $<3.2 \mathrm{pJ} / \mathrm{bit}$. Therefore, for a $\mathrm{BW}$ of $4 \mathrm{~Tb} / \mathrm{s}$, the power consumption is $12.8 \mathrm{~W}$, which corresponds to $84 \%$ energy saving. Another benefit of this approach stems from the improvement of the form-factor of the sub-system from $20 \mathrm{~cm} \times 20 \mathrm{~cm}$ on a PCB to a package size of around $6 \mathrm{~cm} \times 6 \mathrm{~cm}$. The details of the logic memory connection are shown in Figure 5, which schematically shows a TSI that heterogeneously integrates a CPU and 3D memory.

Optical interconnects are known to have the highest bandwidth and lowest power consumption, making them suitable for high bandwidth applications. With future high bandwidth systems, one needs to have optical interconnects to carry data in and out of PCBs that are in server racks. The challenge for using optical interconnects is to bring "optics" as close to CMOS electronics as possible. 2.5D integration using TSI technology can bring optical communication close to high performance CMOS technology-thus allowing servers in datacenters to have the best of both worlds (CMOS technology and optical interconnects). Figure 6 shows another application that benefits from the use of 2.5D TSI technology. It is a high performance line card that may be used in the racks of a server. In this example, an optical fiber connector is used to take data in and out of the line-card. Using 2.5D TSI technology, the PIC and the CMOS drivers can be integrated on the interposer and achieve significant improvements of form-factor. A typical $180 \mathrm{~mm} \times 90 \mathrm{~mm}$ line card can be reduced to a $35 \mathrm{~mm} \times 40 \mathrm{~mm}$ area. The schematic representation of the Electronic -PIC on 2.5D TSI is shown in Figure 6.

One way to implement optical interconnects is to use photonic integrated circuits. Optical interconnects are implemented through continuous wave Distributed Feed Back (DFB) lasers with Si photonics ICs. Heterogeneous integration of electronic and photonic ICs on TSI allows independent technology optimization for Si CMOS processors, memory, CMOS/BiCMOS drivers, and silicon on insulator (SOI) PIC. The integration of Si photonics with TSI will enable the performance requirement of data-centers, super-computers, and high-performance-networking to be met (Fig. 7).

\section{B. General summary in advancement}

Up to this point, we started with how the exploding data traffic in the mobile society is expected to challenge the way we design and manufacture systems. From the application examples shown above, it can be seen that $2.5 \mathrm{D}$ Heterogeneous integration on TSI is a technology platform that can enable system-scaling for the next generation of systems that will be a part of our mobile society. Systems using 2.5D TSI can address both performance and power constraints in a manner that traditional packaging of SoC's built with Moore's law scaling alone cannot. In summary, 2.5D integration allows for a scalable approach to meet power and performance requirements of future generation systems. Heterogeneous integration allows for a manufacturing approach to realize the improvements in power performance metrics that are needed to achieve system scaling for mobile devices, data-centers, and high performance computers. In the rest of this paper, we will highlight the detailed features of this technology to facilitate an understanding of what it takes to design, fabricate, and assemble 2.5D ICs using TSI technology.

\section{Challenges for heterogeneous 2.5D integration on TSI}

While opportunities abound, as is typical with a technology that is on path to wide adoption by industry, 2.5D heterogeneous integration on TSI has yet to address challenges in the design, fabrication, and assembly of $2.5 \mathrm{D}^{9-11}$ and $3 \mathrm{D}$ ICs. ${ }^{17,18}$ In this section, we outline the key challenges faced by TSI technology. Through the course of this paper, we shall elaborate on the same and highlight the techniques and continuing developments that address key challenges.

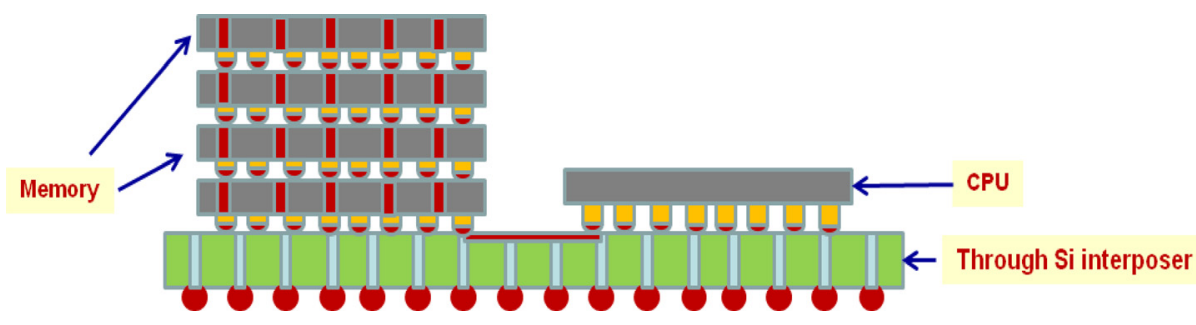

FIG. 5. Cross-section of CPU and 3D memory system showing the die level interconnections. 


\section{Board Level}

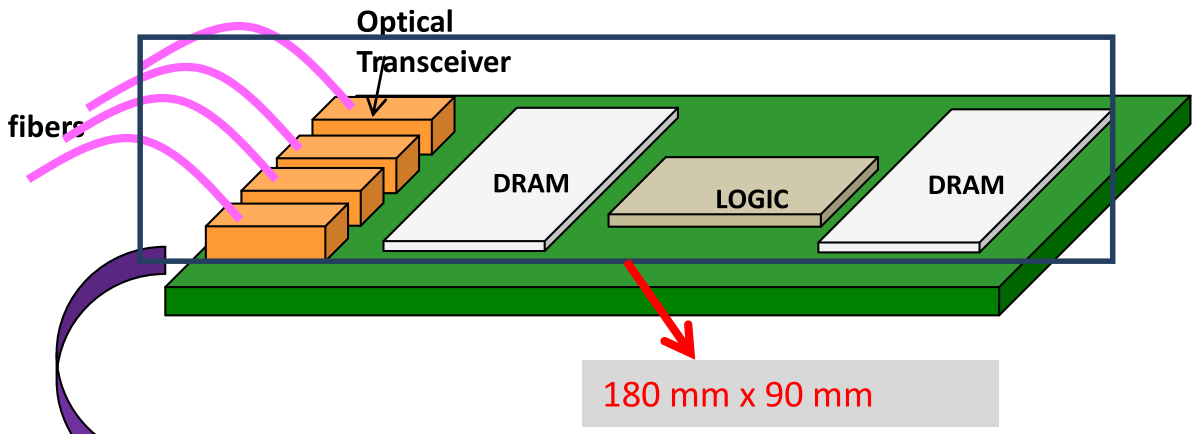

$180 \mathrm{~mm} \times 90 \mathrm{~mm}$

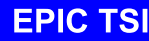

FIG. 6. Electro-photonic integration in TSI in a line card application.
The main technical challenges with designing 2.5D and 3D systems fall in the following areas: (A) Technology planning and design-execution that are necessary for harnessing 2.5D TSI for building systems, (B) processing flow for the fabrication of $100 \mu \mathrm{m}$ thick TSI at acceptable costs, (C) manufacturing flow for assembling multiple ICs on a $100 \mu \mathrm{m}$ thick TSI in a repeatable, and reliable manner, (D) thermal management for addressing heat dissipation of 2.5D TSI, (E) thermo-mechanical analysis and optimization for addressing warpage issues, and $(\mathrm{F})$ electrical characterization and process design kit (PDK) for electronic design automation (EDA) flow of 2.5D TSI.

\section{Technology planning and design-execution}

In designing 2.5D TSI for integrating multiple functions, typically, one needs to start with interconnect planning that would satisfy the design requirements. There are two principle requirements for design engineers: (a) to meet the routing ability requirements of the design and (b) to satisfy the design SI/PI requirements. Routing ability refers to the ability of the TSI to handle the large number of IC-to-IC and IC-to-package interconnections. This is influenced by the available TSI routing area, interconnect size/pitch as well as the number of interconnect layers. More metal layers, larger routing area as well as smaller routing metal size/pitch offer larger routing ability. Increasing the number of metal layers and larger TSI area impose more technology challenges and a concomitant increase in the system cost. In addition, smaller metal size adversely increases the line resistance, giving rise to signal integrity issues. Thus designing a system on 2.5D TSI involves technology planning. For example, there are two fabrication techniques for forming fine-pitch interconnects on a TSI. Typically, two fabrication flows are available to choose from: (1) more expensive $\mathrm{Cu}$-damascene based fine pitch (line width, space $<=1 \mu \mathrm{m}$; Pitch $<=2$ $\mu \mathrm{m}, \mathrm{Cu}$ thickness $\sim 1 \mu \mathrm{m}$ with multiple $>4$ levels of interconnects) and (2) less expensive polymer based $\mathrm{Cu}$ redistribution layer (RDL) flow with coarse pitch (Size $>2$ $\mu \mathrm{m}$; Pitch $>4 \mu \mathrm{m}, \mathrm{Cu}$ thickness of $3 \mu \mathrm{m}$, with up to 4 levels of interconnects). Thus, a designer needs to optimize the choices based on the application needs of routing and SI/PI requirements versus cost of processing. The next step is the physical implementation ${ }^{19}$ of the 2.5D TSI. To do this, one needs accurate electrical models of the TSI (R, L, and C), and a PDK that is implemented on an EDA flow. In addition, testing of $2.5 \mathrm{D} \mathrm{ICs}{ }^{20}$ needs to be addressed as well.

\section{Processing flow for fabrication of $100 \mu \mathrm{m}$ thick TSI}

A key challenge with Si processing for TSI fabrication is with the formation of TSVs which involves etching, dielectric deposition, and $\mathrm{Cu}$ electroplating to fill the TSVs that are typically formed on the front-side of the TSI. Each of these steps needs to be optimized based on TSV density in the TSI. In addition, processing throughput is also a key challenge to ensure cost-containment for these steps. Another challenge arises from handling thinned $300 \mathrm{~mm}$ TSI wafers. Upon thinning, the back-side of the TSI needs specific processing that includes passivation and formation of solder-bumps for attaching to the package substrate. Thin wafer handling is a major challenge in the fabrication of TSI wafers, where industry uses what is called temporary bonding and de-bonding (TBDB).

\section{Manufacturing flow for assembling multiple ICs on a $100 \mu \mathrm{m}$ thick TSI}

Upon fabrication of the TSI, individual ICs need to be attached to the top-side of the TSI. This can typically be done in many ways depending on product requirements. One technique involves singulating the TSI into individual dies, and then performing IC to TSI front-side assembly. This is 
referred to as chip-to-chip (C2C) assembly flow. After this step, the TSI backside is attached to the package substrate (with multiple ICs assembled on the front-side of the TSI). This assembly flow can be time consuming. Therefore, the industry is moving to a chip-to-wafer (C2W) assembly flow where ICs are attached to the TSI while the TSI is still in a wafer form. Such wafer level packaging (WLP) allows for much higher manufacturing efficiency. Both $\mathrm{C} 2 \mathrm{C}$ and $\mathrm{C} 2 \mathrm{~W}$ approaches have challenges in manufacturing-which need to be addressed to ensure high yields and adequate reliability.

\section{Thermo-mechanical analysis and optimization}

The TSI with ICs attached to the front-side is assembled on the package substrate using solder-joints as shown in Figures 4(a) and 4(b). The solder-joint is placed under stress due to the fact that $\mathrm{Si}$ has a low $\left(3 \mathrm{ppM} /{ }^{\circ} \mathrm{C}\right)$ coefficient of thermal expansion (CTE) and the organic (laminate) package has a higher $\left(\sim 16 \mathrm{ppM} /{ }^{\circ} \mathrm{C}\right) \mathrm{CTE}$. The difference in the CTE means that the joined materials expand at different rates during thermal processing and during actual operation when heat is dissipated. This results in solder-joint stress and TSI warpage. Thus, a key challenge in designing a package for 2.5DTSI is to perform accurate thermomechanical analysis/finite element-modeling (FEM), and optimize solder joint placement to ensure reliability of the system.

\section{Thermal management of 2.5D TSI}

With high performance ICs interconnected on the $2.5 \mathrm{D}$ TSI, heat dissipation becomes a challenge. This challenge is commonly referred to as thermal management. Thermal management issues can be understood in two aspects. On one hand, the chip-level power delivery could reach high level such as $100 \mathrm{~W} / \mathrm{cm}^{2}$ for high performance ICs and this requires proximate cooling. Without compromising processing speed, the integration of multi-chips in one interposer on the package tends to generate higher heat density, which in turn can compromise IC performance. On the other hand, the temperature sensitive chips such as photonic ICs prone to wavelength shifts due to the temperature excursions, which need to be thermally controlled. Thermal management uses passive cooling, or active air-cooling or active liquid cooling. As part of this optimization, one needs to design a thermal solution that meets the needs of the end application.

\section{Development of next generation manufacturing equipment}

In order to take $2.5 \mathrm{D}$ TSI technology to high volume manufacturing, improvements in manufacturing efficiencies are needed. As noted earlier, these improvements are focused on WLP to support TSI fabrication and assembly. Key areas where efficiencies are needed include: (a) TBDB for handling thin TSI wafers. Currently, this process has a throughput bottleneck since just a few wafers can be processed per hour through TBDB steps. (b) Chip-to-wafer bondingwhere ICs need to be attached to TSI wafer at high alignment accuracies to support fine pitch $(<40 \mu \mathrm{m}$ pitch) microbumps. Here also, throughput is a key figure of merit.

\section{KEY TECHNOLOGY MODULES FOR 2.5D HETEROGENEOUS INTEGRATION}

Improvement in VLSI performance by downscaling of device dimensions is faced with limitations in system design due to increased power density, higher I/O count, interconnect bandwidth, and timing closure requirements. Si substrate with TSVs or TSI technology is identified as a solution to overcome these challenges. ${ }^{21-23}$ In this section, key $2.5 \mathrm{D}$ TSI technology modules and integration schemes are reviewed. Current development status and readiness of each technology modules and the respective processes will also be discussed in detail.

\section{A. TSV module}

TSV is one of the essential modules in 2.5D heterogeneous integration. This section will focus on the key TSV
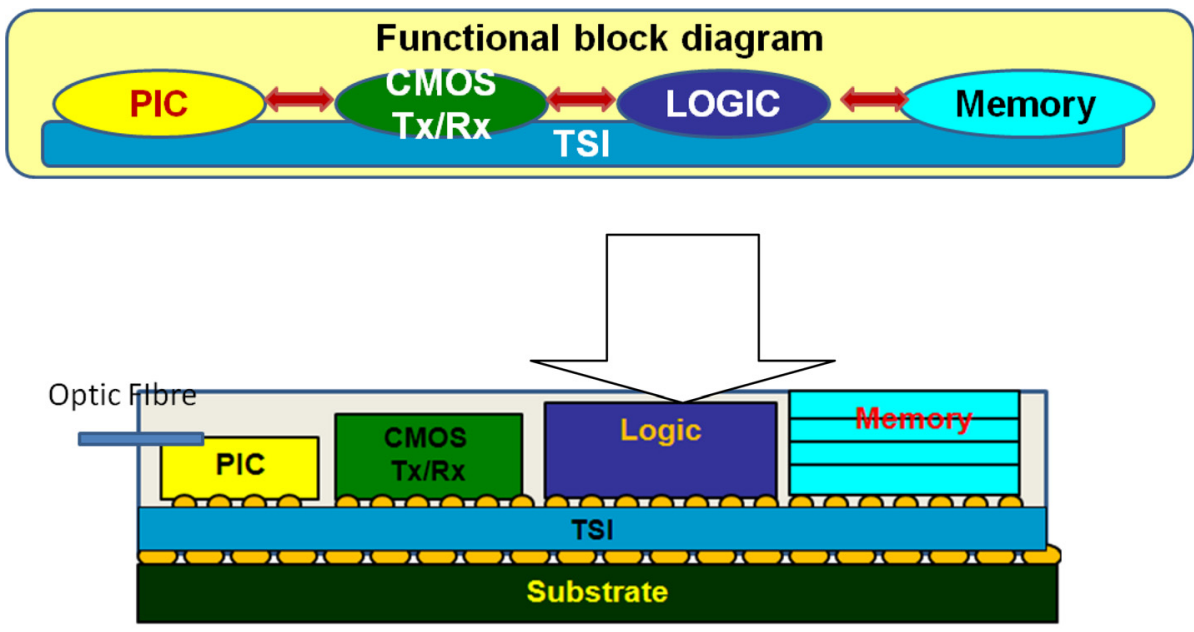

FIG. 7. Functional schematic of electronic-Photonic Integrated Circuits (PIC) on 2.5D TSI.

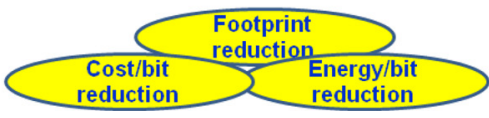


fabrication processes in a typical TSV integration flow (including TSV etch, liner oxide deposition, $\mathrm{Cu}$ barrier seed layer deposition, $\mathrm{Cu}$ electroplating, and Chemical mechanical polishing (CMP)).

First of all, there are many approaches to fabricate TSV. In summary, TSV module fabrication can be classified into three main approaches-TSV via-first (VF), TSV via-middle (VM), and TSV via-last (VL). Each TSV approach is defined by the stage (of a CMOS process flow) at which the TSV module is integrated. For via-first approach, TSV is first developed and fabricated before any device fabrication. For via-middle approach, the fabrication of TSV is done after device (contact) and before back end of line (BEOL) process. For TSV-last approach, the TSV is fabricated after device fabrication and BEOL process. In addition, the integration of the TSV-last approach can be further done in 2 different ways: (1) TSV last integration from the top-side of the wafer and (2) TSV last integration from the backside of the wafer.

In any case, the main TSV process modules include: (a) TSV mask, (b) TSV etching, (c) TSV Photo Resists (PRS) and we clean, (d) liner oxide deposition, (e) barrier and seed layer, (f) Cu plating, and (g) Cu CMP. A typical TSV fabrication process flow is as illustrated in Figure 8.

TSV etching, liner oxide deposition, barrier layer and $\mathrm{Cu}$ seed sputter deposition, Electro-copper-plating (ECP) and $\mathrm{Cu} \mathrm{CMP}$ will be described in subsequence.

\section{TSV deep Si etch process}

TSV etching is a forming of high aspect ratio (AR) $\mathrm{Si}$ etch process. Deep Si etching (from a few tens of to a few hundreds of $\mu \mathrm{m}$ ) is technically more challenging than shallow, low aspect ratio etching in conventional CMOS Si etching process. ${ }^{24}$

As such, high aspect ratio TSV etching faces many challenges. In particular, high etch rate is required for large volume production. Other challenges relating to deep Si etch include the control of sidewall roughness, tilt, sidewall angle, micro-loading, notching, and micro-grass. Currently, a timemultiplexed process, which is more commonly known as
Robert Bosch GmbH's process (BOSCH Process), has become the main TSV etching process adopted by the industry. ${ }^{25,26}$

Bosch etch process is performed using a multiple inductively coupled plasma etching system. It is an alternating two-step process, consisting of a polymerization step and a $\mathrm{Si}$ etch step. The Si etch step rapidly removes the polymer layer on the bottom of the feature while partially removing the polymer layer on the sidewall, protecting the sidewall. Etch and passivation steps are alternated until the desired etch depth is reached. Typically, $\mathrm{SF}_{6}$ and $\mathrm{O}_{2}$ are applied as gas source in the $\mathrm{Si}$ etching cycle while $\mathrm{C}_{4} \mathrm{~F}_{8}$ is applied as polymer generation source during the sidewall passivation cycle. Due to the strong isotropic nature of the $\mathrm{SF}_{6} / \mathrm{O}_{2}$ etch chemistry, each etch cycle will form an unwanted sidewall recess known as a scallop. The formation of this unwanted scallop repeats with each etch cycle. The etch mechanism due to $\mathrm{SF}_{6}$ gases can be explained as follows: ${ }^{26}$

$$
S F_{6}+e^{-} \rightarrow S F_{5}^{+}+F+2 e^{-} .
$$

Usually, $10 \%$ of $\mathrm{O}_{2}$ is added to help in liberating more fluorine radicals in the plasma reaction between $\mathrm{SF}_{6}$ and $\mathrm{O}_{2} . \mathrm{Si}$ is then subsequently etched away by the fluorine according to the following reaction: ${ }^{26}$

$$
\mathrm{Si}(\text { solid })+4 \mathrm{~F}(\text { gas }) \rightarrow \mathrm{SiF}_{4} \text { (gas) } .
$$

During the passivation phase of the $\mathrm{BOSCH}$ process, $\mathrm{CF}_{2}$ is formed from $\mathrm{C}_{4} \mathrm{~F}_{8}$ as follows: ${ }^{26}$

$$
C_{4} F_{8}+e^{-} \rightarrow C_{3} F_{6}+C F_{2}+e^{-} .
$$

$\mathrm{CF}_{2}$ then adsorbs on the surfaces and forms a Teflon-like polymer according to the reaction ${ }^{26}$

$$
n C F_{2} \rightarrow\left(C F_{2}\right)_{n}
$$

During the etch phase, the $\mathrm{CF}_{2}$ molecules are removed by a combination of physical ion sputtering and chemical reactions. The physical component in the Bosch process is contributed by the positively charged $\mathrm{S}_{\mathrm{x}} \mathrm{F}_{\mathrm{y}}{ }^{+}$, and the chemical etch is mainly due to the $\mathrm{F}$ and $\mathrm{O}_{2}$, which are generated
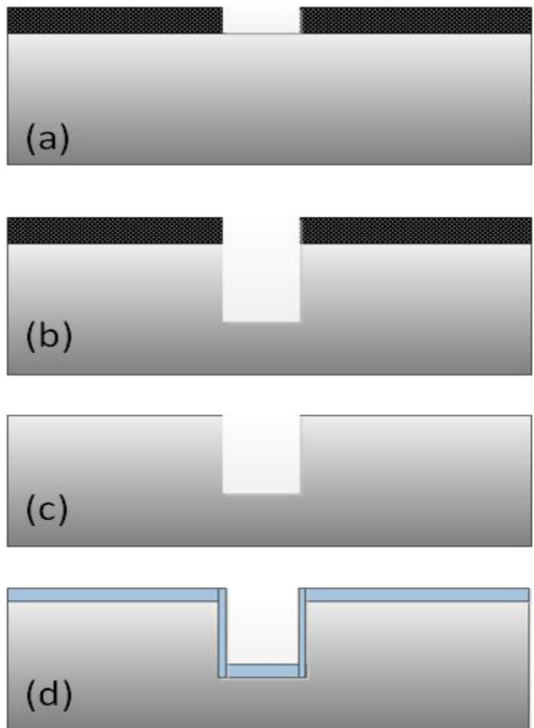

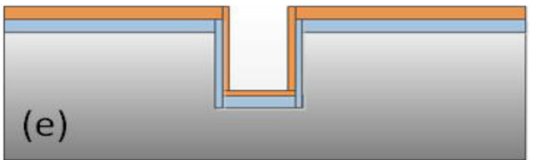

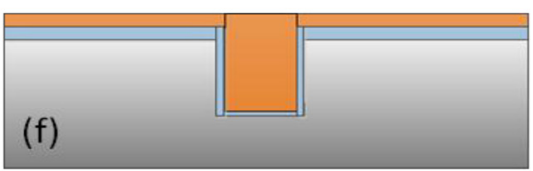
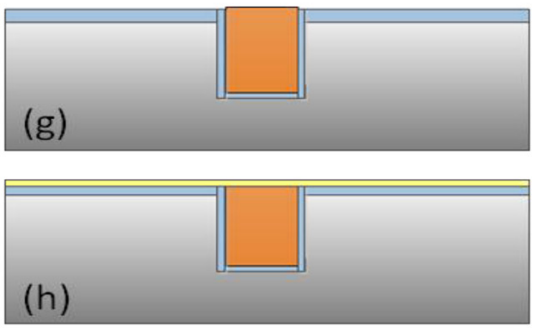

FIG. 8. Major process modules in TSV formation. (a) TSV mask lithography, (b) deep Si etch, (c) PRS and wafer cleaning, (d) liner oxide deposition, (e) barrier metal and $\mathrm{Cu}$ seed sputtering, (f) $\mathrm{Cu} \mathrm{ECP}$, (g) $\mathrm{Cu} \mathrm{CMP}$, and (h) TSV capping deposition if needed. 


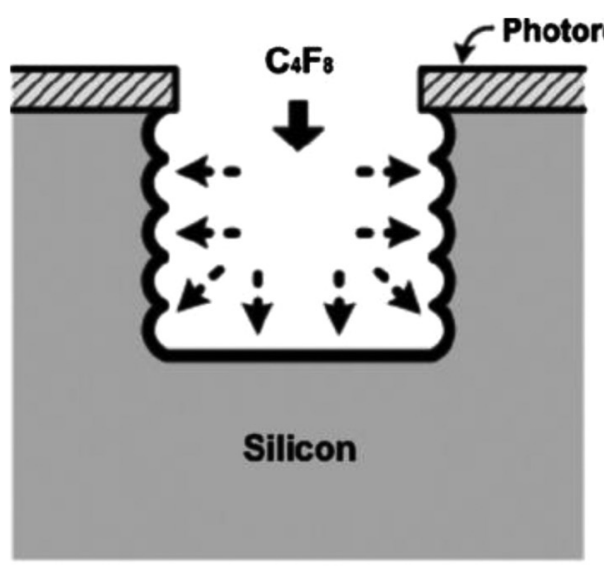

(a)

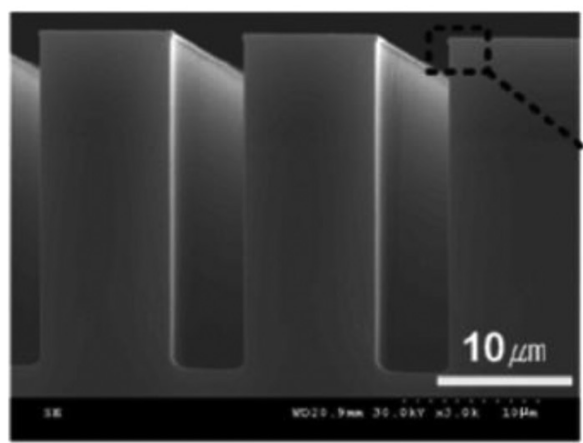

(c)

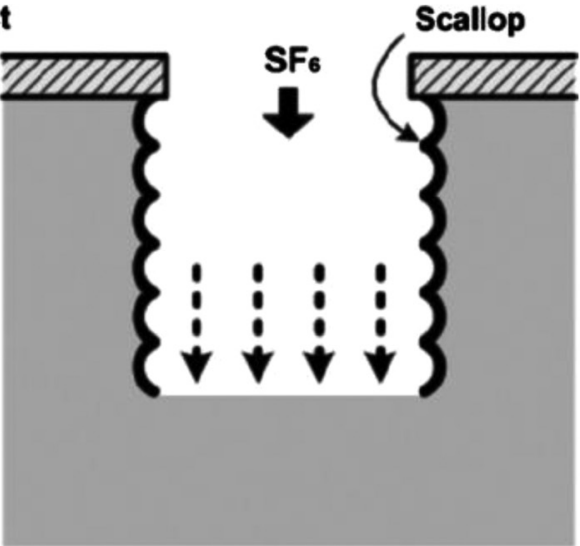

(b)

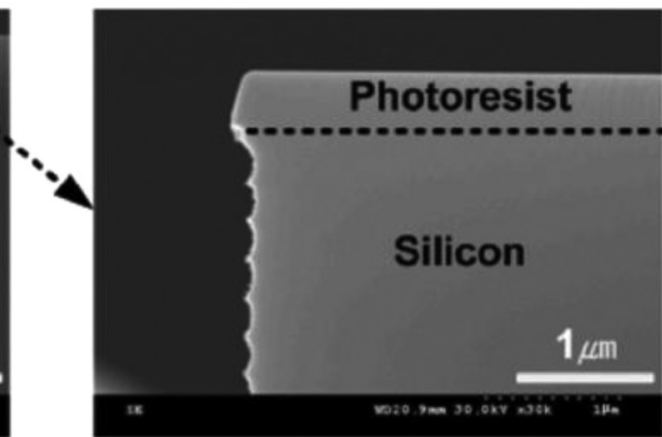

(d)
FIG. 9. Schematic of Bosch method: (a) sidewall passivation using $\mathrm{C} 4 \mathrm{~F} 8$, (b) Si isotropic etching using SF6, (c) SEM images of deep trenches, and (d) magnified SEM image of the sidewall showing nano-scallops. ${ }^{27}$ during each etch step. The scallops are due to the isotropic etching mechanism as shown in reaction (2). A schematic of the Bosch etch process is illustrated in Fig. 9. ${ }^{27,28}$ Figure 10 shows a $10 \mu \mathrm{m}$ diameter by $100 \mu \mathrm{m}$ depth TSV etch profile obtained via Bosch etch process.

Although Bosch process is able to meet the requirements of a TSV etch, there are still issues associated with the process. Some issues in TSV Bosch etch include micro-loading effect, undercut (also known as over-hang), Si grass, undercut, and notching. Wu et al did a detailed review on the loading effect and $\mathrm{Si}$ micro-grass for TSV etching, with corresponding proposed solutions. ${ }^{27}$ The undercut issue had been observed more frequently in via-middle scheme ${ }^{29}$ and TSV in SOI wafers. ${ }^{30}$ Figure 11 shows SEM images of undercut issues in TSV fabrication. Both undercut and scallop will result in liner coverage uniformity issues, barrier metal and $\mathrm{Cu}$ seed step coverage issues, which will then cause incomplete TSV filling or leave voids inside TSVs. This effect becomes more pronounced as the TSV diameter gets smaller. On the other hand, notching is normally observed in via-last scheme when the etching gas hits the boundary between $\mathrm{Si}$ and the underlying layer, as shown in Figure $12 .^{27}$ The reason is charge accumulation that occurs where $\mathrm{Si}$ is underlain by a dielectric layer. Increasing the thickness of polymer during over etch by increasing is proposed to prevent notching issue. $^{31,32}$ Another method to control notching is to apply intermittent or pulsed bias RF power to cathode. ${ }^{3,34}$

The post-etch cleaning of the TSV is a critical process. In particular, the F-containing polymers deposited during the passivation cycle of a Bosch process have to be removed fully before downstream process

\section{TSV liner deposition}

TSV liner deposition is a process that is used to deposit a layer of dielectric film, e.g., typically $\mathrm{SiO}_{2}$, along the sidewalls of the deep $\mathrm{Si}$ via, and it is used as an electrical

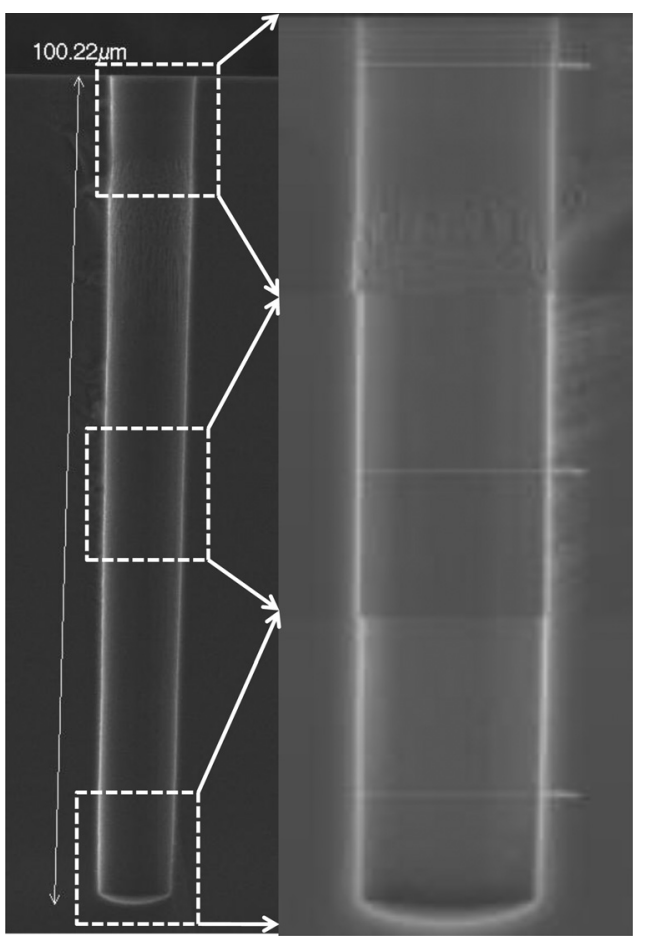

FIG. 10. TSV profile after deep reaction ion etching by using Bosch method. The size of TSV shown in the figure is $10 \mu \mathrm{m}$ in diameter and $100 \mu \mathrm{m}$ in depth, which has been widely accepted for interposer application. 


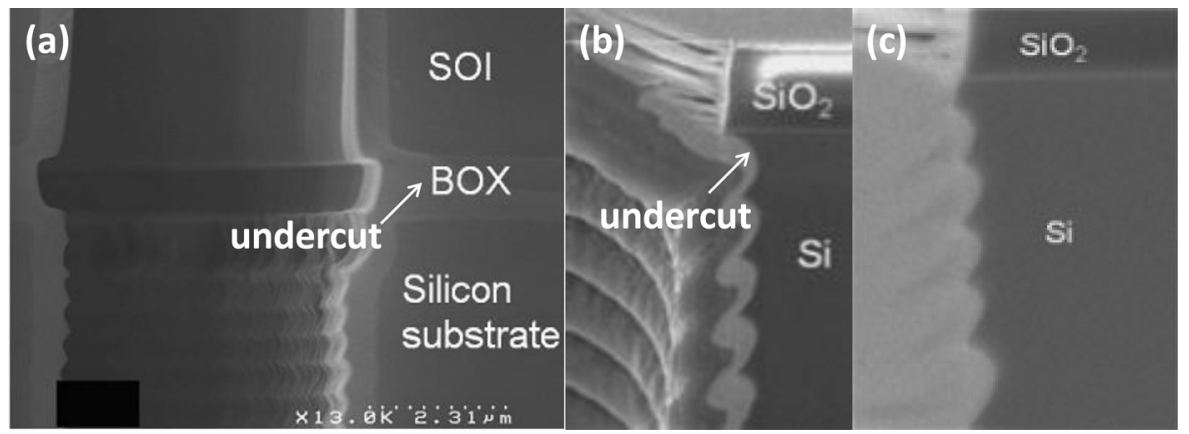

FIG. 11. Undercut issues of TSV etching. (a) Undercut of TSV in SOI wafer, ${ }^{30}$ (b) undercut of TSV in viamiddle scheme, ${ }^{29}$ and (c) improved etching recipe showing less undercut. isolation for the TSV structure. It can be done either by using high temperature thermal oxide deposition or by plasma enhanced chemical vapor deposition (PECVD) with the use of silane and tetrathylorthosilicate (TEOS). ${ }^{35,36}$ There is wide application of Sub-atmospheric CVD (SACVD) of Ozone (O3)-TEOS as liner in TSV formation. ${ }^{37-39}$ Different TSV approaches have different requirements. But the basic requirement that is expected for liner deposition is good step coverage for the TSV insulation from the Si substrate. Thermal oxide is used in the TSV first approach because high temperature process will not have any effect on the active devices since the TSV is fabricated before the front end of line (FEOL) process. SACVD oxide is commonly used in the TSV middle and via last from top-side approach because the active devices are fabricated before the TSV integration. High liner deposition temperature can affect the performances of the active devices in via middle approach and the BEOL in the TSV last from top-side approach. The type of pre-cursor that is more commonly used in the SACVD process for the TSV middle and TSV last from top integration is TEOS. The reason being that using TEOS (as the pre-cursor) will give a better $\mathrm{SiO}_{2}$ step coverage than silane (as the pre-cursor) in the PECVD process. Low temperature $\left(<250^{\circ} \mathrm{C}\right)$ TEOS-PECVD is normally used in the TSV VL from backside approach due to the thermal budget limitation of the type of bonding material used. SEM images of TSV liner oxide at different depth are shown in Figure 13, and Table I summarizes the liner oxide step coverage (SC) at various TSV depths and wafer locations.

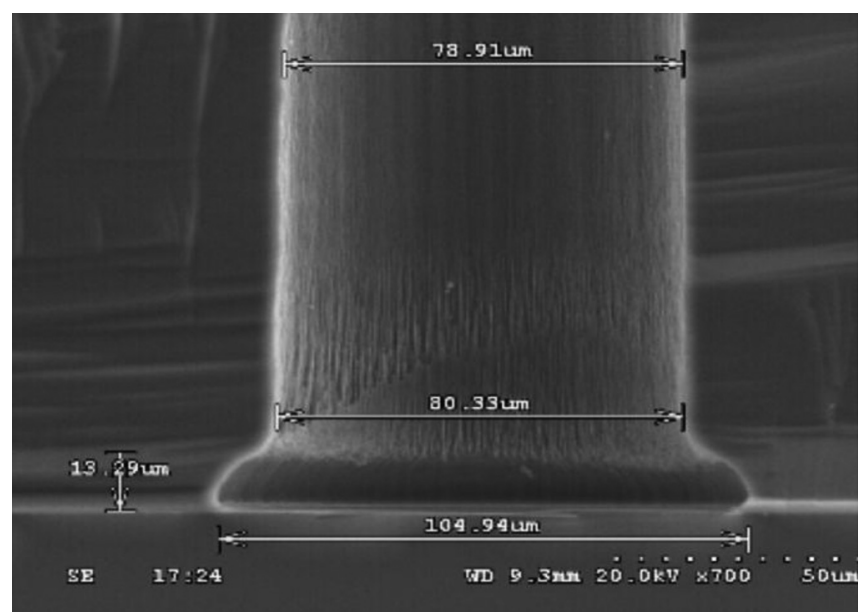

FIG. 12. Notching observed at the interface between $\mathrm{Si}$ and $\mathrm{SiO}_{2}$ layer. $^{27}$

\section{Seed layer and barrier layer deposition}

Next key process after liner deposition in TSV fabrication is the barrier and seed layer deposition. The purpose of the barrier layer is to prevent any copper $(\mathrm{Cu})$ diffusion from the TSV into the Si during any subsequent high temperature process. The deposition of the barrier and seed layer is normally carried out using a chemical or physical vapor deposition (CVD or PVD). ${ }^{40}$ CVD-based process technology (e.g., metal organic chemical vapor deposition (MOCVD)) can cater for barrier and seed deposition in very high aspect ratio $(>15: 1)$ TSVs but incurs higher cost and is not the mainstream process in the industry. Conventional PVD process is less costly but poses more limitations with respect to step coverage on the TSV side wall. Improvements in PVD equipment have extended the process window for PVD barrier and $\mathrm{Cu}$ seed layer deposition. ${ }^{39,40}$ Tantalum (Ta) and $\mathrm{Ti}$

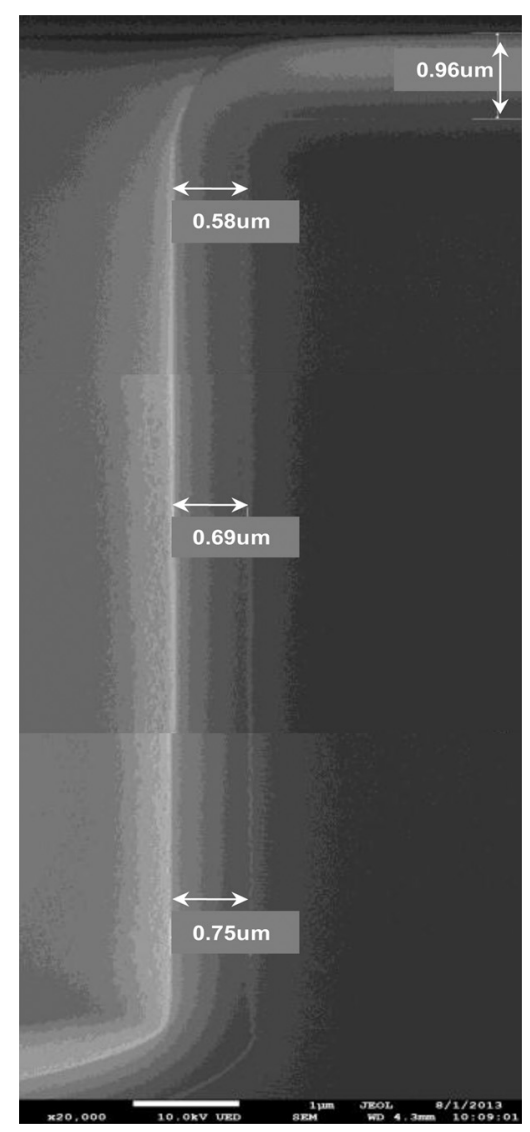

FIG. 13. SEM images of O3-TEOS liner oxide for a TSV with the diameter of $10 \mu \mathrm{m}$ and depth of $100 \mu \mathrm{m}$. 
TABLE I. Summary of the liner thickness in various TSV depths and die locations.

\begin{tabular}{|c|c|c|c|c|c|c|c|c|c|c|}
\hline Die location & $\begin{array}{l}\text { Field } \\
(\mu \mathrm{m})\end{array}$ & $\begin{array}{l}\text { Invia field } \\
(0.21 \mu \mathrm{m})\end{array}$ & $\begin{array}{l}\text { Top sidewall } \\
(\mu \mathrm{m})\end{array}$ & $\mathrm{SC}(\%)$ & $\begin{array}{l}\text { Middle sidewall } \\
\qquad(\mu \mathrm{m})\end{array}$ & $\mathrm{SC}(\%)$ & $\begin{array}{l}\text { Bottom sidewall } \\
\qquad(\mu \mathrm{m})\end{array}$ & $\mathrm{SC}(\%)$ & $\begin{array}{l}\text { Bottom } \\
(\mu \mathrm{m})\end{array}$ & $\mathrm{SC}(\%)$ \\
\hline Bottom & 0.95 & 0.74 & 0.62 & 83.8 & 0.69 & 93.2 & 0.77 & 104.1 & 0.75 & 101.4 \\
\hline Center & 0.98 & 0.77 & 0.58 & 75.3 & 0.73 & 94.8 & 0.73 & 94.8 & 0.71 & 92.2 \\
\hline Edge & 0.92 & 0.71 & 0.62 & 87.3 & 0.69 & 97.2 & 0.74 & 104.2 & 0.73 & 102.8 \\
\hline Left & 0.93 & 0.72 & 0.6 & 83.3 & 0.68 & 94.4 & 0.75 & 104.2 & 0.73 & 101.4 \\
\hline Middle & 0.96 & 0.75 & 0.58 & 77.3 & 0.69 & 92.0 & 0.75 & 100.0 & 0.75 & 100.0 \\
\hline Top & 0.92 & 0.71 & 0.64 & 90.1 & 0.73 & 102.8 & 0.78 & 109.9 & 0.78 & 109.9 \\
\hline
\end{tabular}

are prevalent barrier metals used. These barrier layers also have the function of improving the adhesion between TSV $\mathrm{Cu}$ and the liner dielectric. After barrier deposition, $\mathrm{Cu}$ seed is sputtered in a separate chamber. Ar plasma is first applied to the metal target that is used for sputtering. The metal atom is then dislodged from the metal target and deposited onto the bottom wafer surface to form the metal film. SEM images of a PVD barrier metal and $\mathrm{Cu}$ seed at various depth in a $10 \mu \mathrm{m} \times 100 \mu \mathrm{m}$ TSV are shown in Figure 14 .

The continuity of barrier metal and $\mathrm{Cu}$ seed is the basic and most essential requirement of the PVD process. As shown in Figure 14, the barrier metal and $\mathrm{Cu}$ seed fully covers the side-walls surface within the TSV. Ti step coverage can achieve $0.63 \%-5.97 \%$ at different depth of TSV. PVD $\mathrm{Cu}$ has better step coverage compared with Ti. Typical step coverage of $\mathrm{Cu}$ is about $1.06 \%-6.03 \%$ in a $10 \mu \mathrm{m} \times 100 \mu \mathrm{m}$ TSV.

\section{4. $E C P$}

ECP process is another important process step in the fabrication of a TSV. The purpose of this step is to fill up the deep Si etched TSV with either copper or other types of conducting materials (aluminum or tungsten) for the TSV. Via plating defects can have various negative effects on the TSV interconnect properties and subsequent TSV processes. Voids in the TSV fill can cause variations in via resistance, or even electrically open-circuit connections.

ECP is a plating process in which metal ions in a solution is moved by an electric field to coat an electrode. ${ }^{41} \mathrm{ECP}$ can be done using either by conformal or bottom-up deposition methods, and that will depend on the types of chemistry and electroplating conditions that are used. Conformal $\mathrm{Cu}$ deposition for the TSV is fast but will result in a high $\mathrm{Cu}$ overburden in the field region and a potential high risk of voids in the TSV. On the other hand, bottom up plating method has lower risk of void formation, and there will be less $\mathrm{Cu}$ overburden even though the process time is long. A TSV ECP process encompasses a combination of both conformal and bottom-up methods.

The ECP bath chemistry plays an important role in the TSV filling process. The presence of organic additives in the bath chemistry has strong influence on the $\mathrm{Cu}$ deposition process. In summary, there are three main types of organic additives applied to optimize the process of TSV fillingsuppressor, accelerator, and leveler. Suppressor is a long

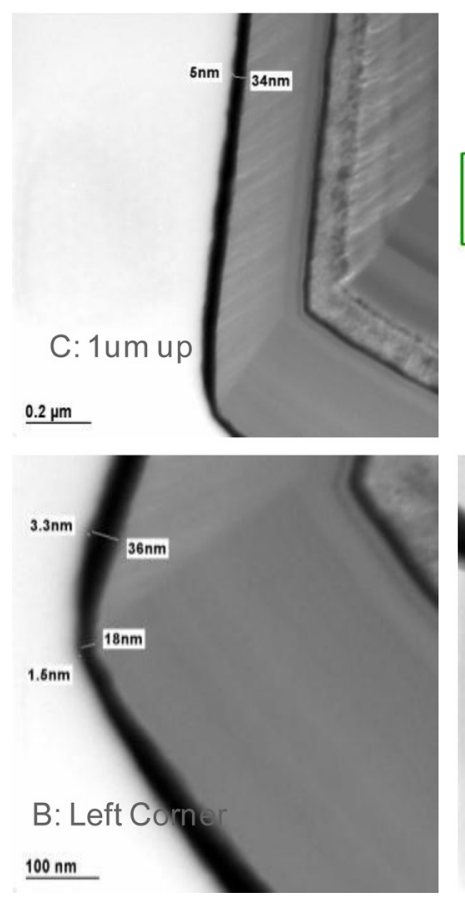

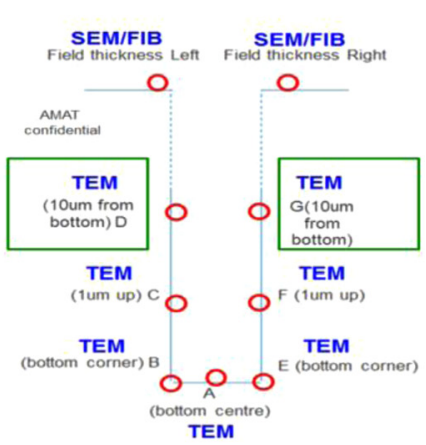
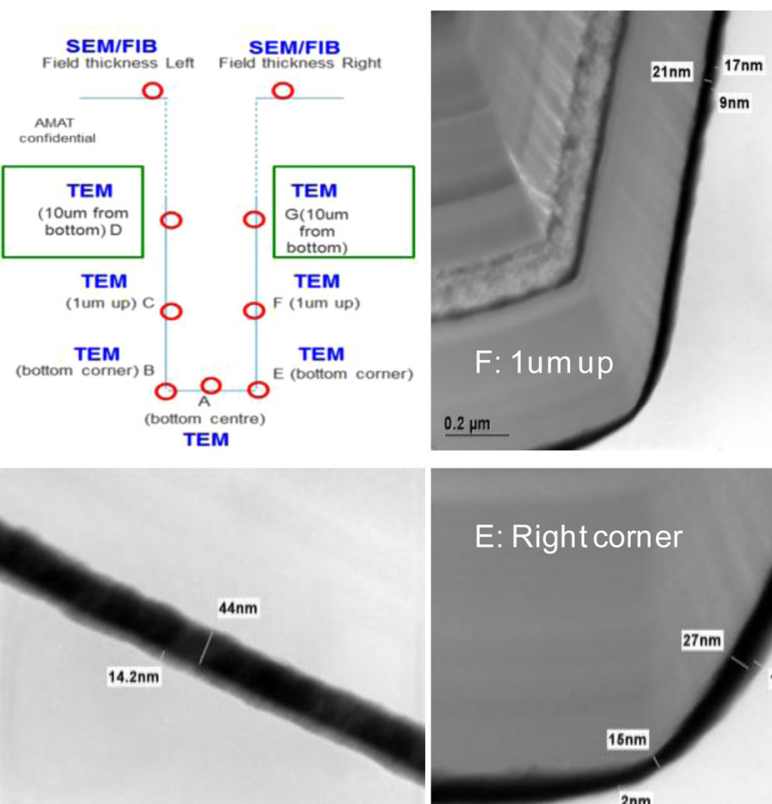

A: Bottom

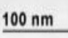

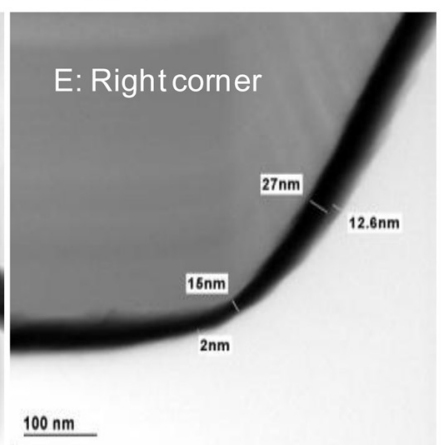
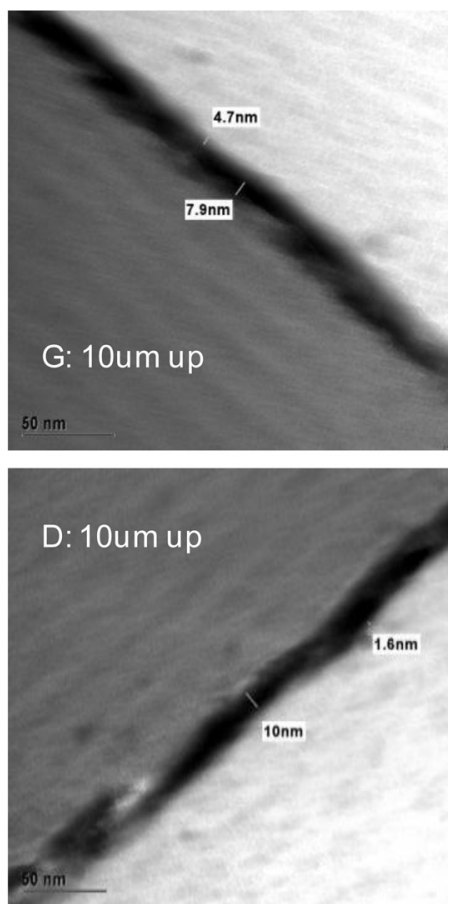

FIG. 14. SEM images of Ti and $\mathrm{Cu}$ as barrier and seed layer for $10 \mu \mathrm{m} \times 100 \mu \mathrm{m} \mathrm{TSV}$. 

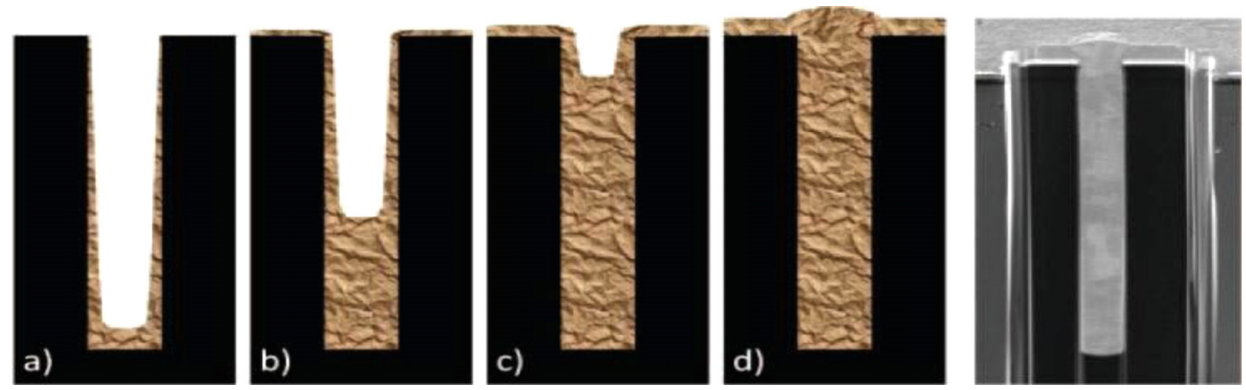

FIG. 15. Cu filling process to form a void-free TSV. (a) Initial stage, (b) intermediate stage, (c) final stage, and (d) void-free $\mathrm{Cu}$ filling and the image of actual TSV after $\mathrm{Cu}$ filling. chain polymer and is usually used to coat the $\mathrm{Cu}$ surface to create a "blocking layer" during $\mathrm{Cu}$ deposition. Accelerator is a catalyst that is used during the $\mathrm{Cu}$ deposition process to increase the $\mathrm{Cu}$ deposition rate. A leveler is applied to deactivate the effect of the accelerator. ${ }^{41}$

Nowadays, the TSVs are mostly filled with $\mathrm{Cu}$ by ECP process. This step is considered as the most critical step for TSV formation, because it determines if the $\mathrm{Cu}$ filling is successful. If there are any issues in the process, $\mathrm{Cu}$ filling defects, also known as voids, will be observed inside the TSVs. Figure 15 shows the conventional model of bottom up filling for void-free TSV $\mathrm{Cu}$ filling when all processes are controlled well. ${ }^{42}$ Choi et al. $^{42}$ and Malta et al. ${ }^{43}$ have studied and investigated the mechanism of failure and defect generation of TSV filling during ECP process, to realize a low-cost 3D IC based on the TSV. X-ray is a non-destructive method employed to inspect for voids in TSVs. Figure 16 shows X-ray and X-SEM images of TSV with $10 \mu \mathrm{m}$ in diameter and $100 \mu \mathrm{m}$ in depth. Throughput and $\mathrm{Cu}$ overburden reduction remain as key challenges to be addressed for cost saving. ${ }^{44}$ A thick $\mathrm{Cu}$ overburden will not only affect overall throughput but also cause failures due to the serious warpage. In the case of a $10 \times 100 \mu \mathrm{m}$ TSV, a well-optimized TSV $\mathrm{Cu}$ filling process by electroplating should typically have a $\mathrm{Cu}$ overburden thickness of below $4 \mu \mathrm{m}$. In addition, because the $\mathrm{Cu}$ overburden thickness depends on the TSV dimensions, it can be much thicker for bigger dimensions of TSV applications.

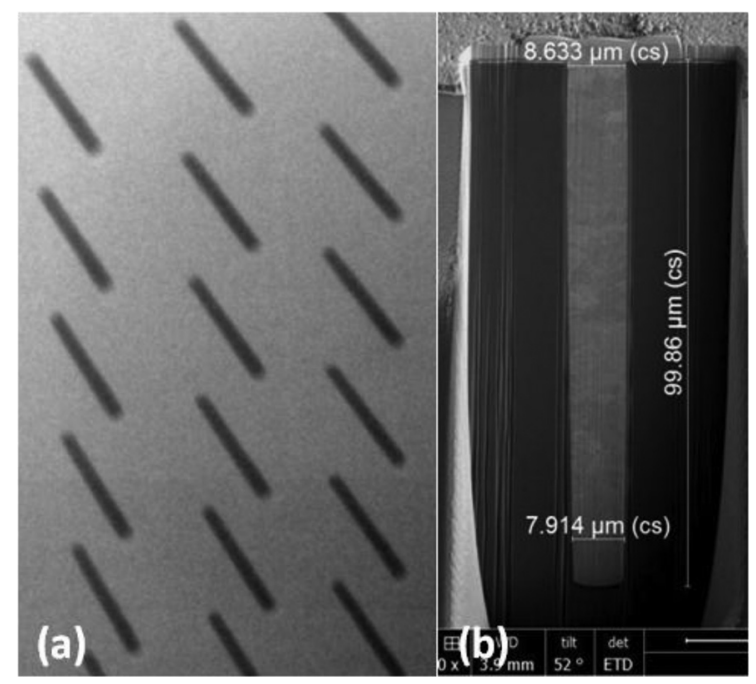

FIG. 16. X-ray (a) and X-SEM images (b) of $10 \mu \mathrm{m} \times 100 \mu \mathrm{m}$ TSV after ECP.

\section{CMP}

After the ECP process of the TSV, CMP has to be carried out to remove any $\mathrm{Cu}$ overburden and $\mathrm{Cu}$ mounts that have occurred during the ECP process. ${ }^{45,46}$ The Cu TSV will have to be exposed by using a CMP process in order to proceed with further process integration.

Conventional $\mathrm{Cu}$ CMOS process needs to go through a high temperature annealing step before $\mathrm{Cu} \mathrm{CMP}$ in order to achieve $\mathrm{Cu}$ grain stability. Because most of TSV wafers have thicker $\mathrm{Cu}$ overburden, e.g., $>4 \mu \mathrm{m}$, two-step $\mathrm{Cu} \mathrm{CMP}$ process are employed for surface planarization. Higher $\mathrm{Cu}$ overburden will cause wafer to have a higher warpage after TSV Cu CMP. The first copper CMP process is carried out right after copper ECP process, the wafer is then sent for $\mathrm{Cu}$ annealing, followed by a second $\mathrm{Cu} \mathrm{CMP}$ process to remove the remaining $\mathrm{Cu}$ protrusion and $\mathrm{Cu}$ annealing defects. The mechanisms involve in the CMP process of different types of metals are relatively simple. In general, an oxide of the metal is chemically formed on the metal film surface. It is then removed mechanically with abrasives in the CMP slurry. ${ }^{46} \mathrm{~A}$ hybrid e-CMP/CMP has been proposed by Takeda and coworkers $^{47}$ and applied for Cu TSV CMP, and this method shows higher $\mathrm{Cu}$ removal rate as well as minimum dishing of $100 \mathrm{~nm}$ for TSVs with $100 \mu \mathrm{m}$ diameters. Figure 17 shows typical images of TSV after CMP process.

\section{B. BEOL and RDL scheme}

For fine pitch and high density TSV interposer, industry is looking at multiple interconnect layers on the interposer front side with less than $5 \mu \mathrm{m} / 5 \mu \mathrm{m} \mathrm{L/S}$ and via diameter

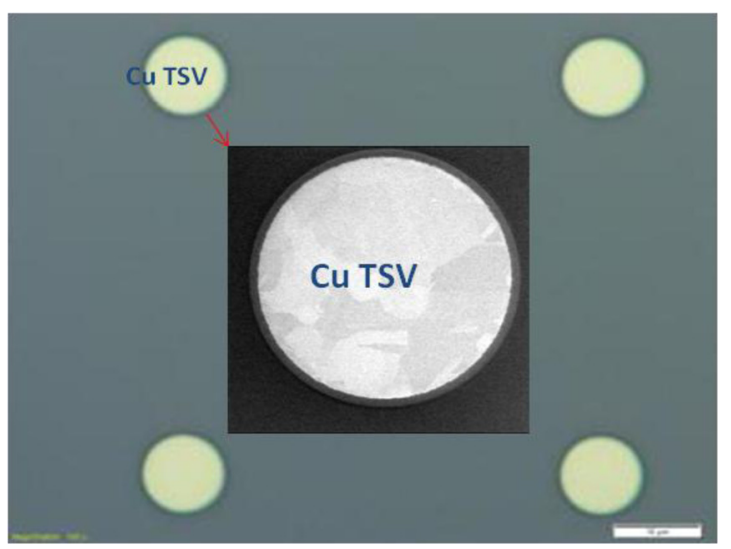

FIG. 17. Optical inspection and SEM inspection (inset) results of $10 \mu \mathrm{m} \times 100 \mu \mathrm{m}$ TSV after CMP process. 
less than $10 \mu \mathrm{m}$. To achieve fine pitch $\mathrm{Cu}$ routing in through $\mathrm{Si}$ interposer, the main stream technology adopted is foundrybased BEOL process. ${ }^{48} \mathrm{BEOL}$ fabrication is based on single or dual $\mathrm{Cu}$ damascene process with inorganic dielectric materials, e.g., $\mathrm{SiO}_{2}, \mathrm{Si}_{3} \mathrm{~N}_{4}$, or low-K materials. In damascene process, the underlying dielectric insulator material is patterned and etched with open trenches and vias and a thick layer of $\mathrm{Cu}$ is deposited to overfill the etched structures. CMP is then used to remove the $\mathrm{Cu}$ overburden leaving $\mathrm{Cu}$ filled in the trenches. $\mathrm{Cu}$ remaining in the trench forms the conducting traces.

Compared to damascene process, polymer-based $\mathrm{Cu}$ RDL process offers lower cost interconnect solution, since it avoids deep ultraviolet (DUV) lithography, plasma etching, and CMP process. The RDL process involved using a semiadditive process to form $\mathrm{Cu}$ conducting traces and photo-pattern-able polymer materials are used as the dielectric insulating layer. The contact via opening can be formed directly in the polymer material by photo-lithography and developing the material away. Polymer-based Cu RDL process offers a cost effective interconnect solution, since it avoids DUV lithography, plasma etching, and CMP process. As shown in Figure $18,{ }^{49}$ process step count of polymer-based RDL is reduced by $\sim 45 \%$ as compared with dual-damascene process, and $\sim 60 \%$ as compared with single damascene process. However, current polymer-based $\mathrm{Cu}$ RDL processes are normally coarse in terms of L/S and photo-lithography resolution of photo-dielectric materials are often very poor in achieving very small contact via opening. Fine-pitch polymer-based RDL process is becoming more in demand under the pressure of cost reduction of $2.5 \mathrm{D}$ interposer and 3D-IC.

RDL process is a standard process in the packaging area. ${ }^{50,51}$ IME has been developing RDL process for packaging application with wider dimension. ${ }^{52}$ Later, low temperature damascene interconnect has been developed for 3D interconnect. ${ }^{53}$ Shrinkage of RDL has become a popular topic of interest recently. Spin-on photo-dielectric based $\mathrm{Cu}$ RDL integrated with TSV interposer was reported with $4 \mu \mathrm{m} / 4 \mu \mathrm{m}$ line/space $(\mathrm{L} / \mathrm{S})$ and $10 \mu \mathrm{m}$ in via diameter by Kumagai et al., ${ }^{50}$ and Ho et al. reported $2 \mu \mathrm{m} / 2 \mu \mathrm{m} \mathrm{L} / \mathrm{S}$ RDL with $2 \mu \mathrm{m}$ polymer via diameter. ${ }^{49}$ Taiwan Semiconductor Manufacturing Company, Limited (TSMC) reported $0.8-\mu \mathrm{m} /$ $0.8-\mu \mathrm{m} \mathrm{L} / \mathrm{S}$ RDL in VLSI $2014 .^{54}$ The shrinkage of RDL has become an important research direction in the foundry for cost reduction in TSI application.

$2 \mu \mathrm{m} / 2 \mu \mathrm{m}$ line/space (L/S) RDL was developed and characterized in Ref. 49. Figure 19 shows the cross-section of 2 layers polymer-based RDL meander (a) and daisy chain (b) and (c) structures fabricated on Si wafer. Metal 1 layer consists of $2 \mu \mathrm{m} / 2 \mu \mathrm{m} \mathrm{L} / \mathrm{S} \mathrm{Cu}$ traces fabricated using the semi-additive process. Metal 2 layers consist of $2.5 \mu \mathrm{m}$ line width $\mathrm{Cu}$ trace with $1.5 \mu \mathrm{m} / 1.5 \mu \mathrm{m}$ line/space in Figure 19(a). The 2 layers are insulated by polymer-based dielectric material. The spin-on polymer-based material shows good gap-filling capability with no voids observed in between the line space. The daisy chain consists of metal 1 and 2 with

\section{Polymer-based RD

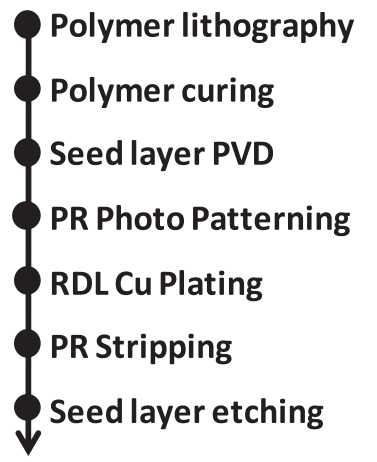

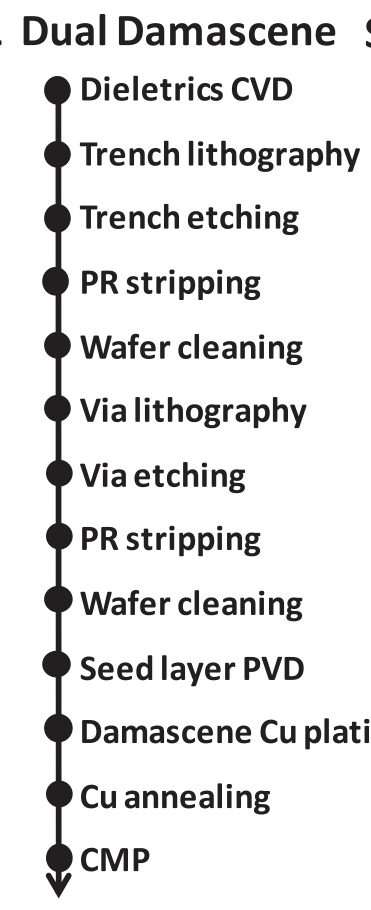

Single Damascene

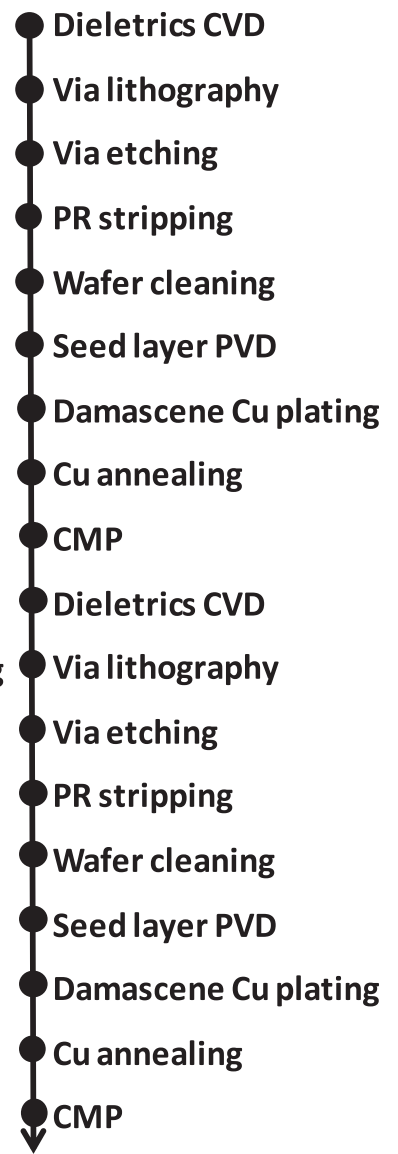

FIG. 18. Comparison of process steps of different $\mathrm{Cu}$ wiring processes, i.e., polymer-based RDL, dual damascene, and single damascene. 


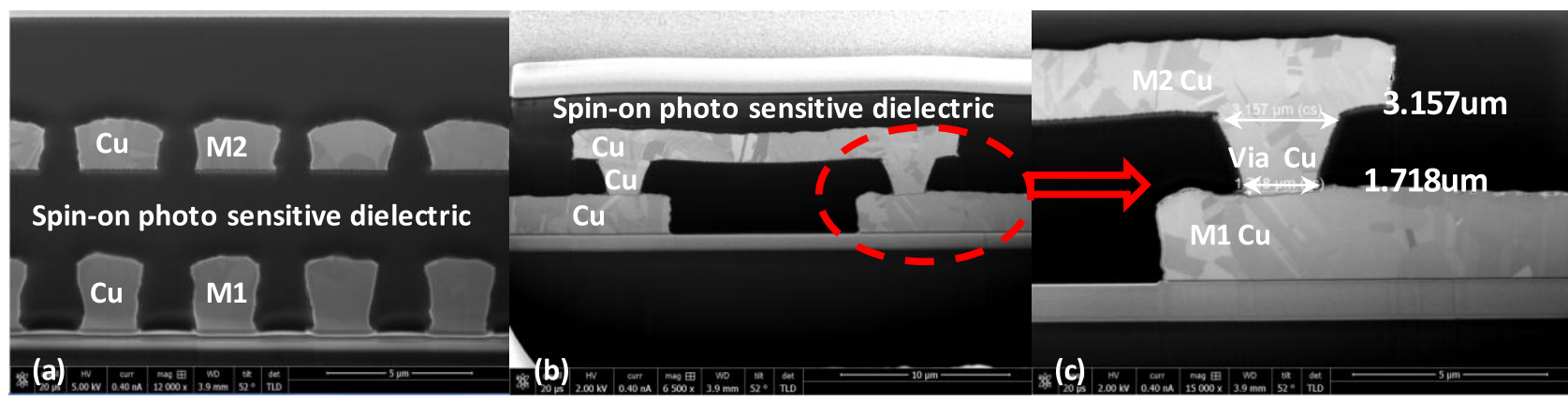

FIG. 19. Cross-section of (a) 2 layer fine pitch RDL meander, (b) daisy chain structures, and (c) enlarged view of via.

vias connecting the 2 metal layers in Figure 19(b). The contact via is enlarged and shown in Figure 19(c) that has a tapered slope profile. The top opening diameter for the contact via is $\sim 3.2 \mu \mathrm{m}$, and the bottom diameter is $\sim 1.8 \mu \mathrm{m}$. The tapered slope via sidewall is preferred in RDL processing as it allows better step coverage of the PVD seed layer.

\section{Bumping}

\section{Solder bumping and Cu pillar bumps}

Solder bumping technology (the process of joining a chip to a substrate without shorting using solder) is first conceived and implemented by IBM in the early 1960s. Fine pitch solder micro-bumps are one of the key elements to form high density interconnections on a through $\mathrm{Si}$ interposer or functional dies. To meet Tera-scale computing needs and to reduce power consumption for next generation information devices, short and high density interconnections between high-capacity memory chips and microprocessor unit are required. ${ }^{55-57}$ In order to achieve interconnections with low resistance, most frequently solder micro-bumps are made up of $\mathrm{Cu}$ and a solder cap, i.e., $\mathrm{Cu}$ pillar bumps. Figure 20 shows the evolution map of $\mathrm{Cu}$ pillar bumps. Table II below shows summarization of bumping technology for chip to chip interconnects in terms of scaling and bonding technology.

As compared to solder bumps, the pillar bumps are more rigid and thus allow assembly with only a small solder volume on top of pillars. This results in two benefits: (1) the standoff can be kept high even with very fine pitch, and (2) the solder will not spread out much on wettable surfaces Nowadays, fine pitch $\mathrm{Cu}$ pillar bumps are applicable in various packaging technologies, from wire bonded chip stacking to chip stacking with TSVs, from system in package (SiP) to 3D IC integration. As a result, $\mathrm{Cu}$ pillar bumping technology has attracted intensive attentions. As to the assembly, different metallurgical bonding processes have been developed, such as solid-liquid-inter diffusion method ${ }^{58}$ and thermal compression method. ${ }^{59,60}$ In IBM, Wright et al. developed solder micro bumping technology. ${ }^{61}$ Different solder materials, such as e-PbSn and CuSn systems, have been studied.

Fine-pitch $\mathrm{Cu}$ pillar bumps have replaced conventional flip chip solder bumps driven by the need for extremely low profile and high connectivity interconnects. Devices, such as high-end processors, graphics, FPGAs, power amplifiers, MEMS, and High brightness light emitting diode (HB-LED), have incorporated fine-pitch $\mathrm{Cu}$ pillar bumps and demonstrated the range of the technology. Figure 21 shows four SEM images of $40 \mu \mathrm{m}$ pitch copper pillar bumps capped with SnAg solder. ${ }^{62}$ The SnAg solder reflows to join the die to the $\mathrm{Si}$ interposer. Sub- $40 \mu \mathrm{m}$ pitch has also been demonstrated.

\section{Bumps in 2.5D TSI package}

To support high speed data transfer and preserve signal integrity between dies, a very fine-geometry circuit board and enabling interconnect solution is required. TSIs and finepitch $\mathrm{Cu}$ pillar bumps are two representative technologies that have played an integral role in defining the $2.5 \mathrm{D}$ package approach. Figure 22 shows the schematic of a typical 2.5D TSI system with two-die side-by-side integration. Interposer inclusion defines the $2.5 \mathrm{D}$ approach. It routes thousands of interconnections between the devices. IC1 and IC2 can also serve as TSV-enabled 3D stacks. As shown in Fig. 21, Cu pillar microbumps provide short, low inductance, efficient interconnections (a) between ICs in vertical stacks as well as (b) between and IC and the through Si interposer. Together, the micro-bumps and Si interposer provide a highspeed and high-bandwidth communication high way for side-by-side die (and stack) placement. The interposer bottom fans out to wider pitches that employ conventional solder bumps for connection onto the ball grid array (BGA) substrate.

\section{Fabrication of Cu pillar micro-bumps}

The structure and production process of a pillar bump are very similar to an electro-plated solder bump.
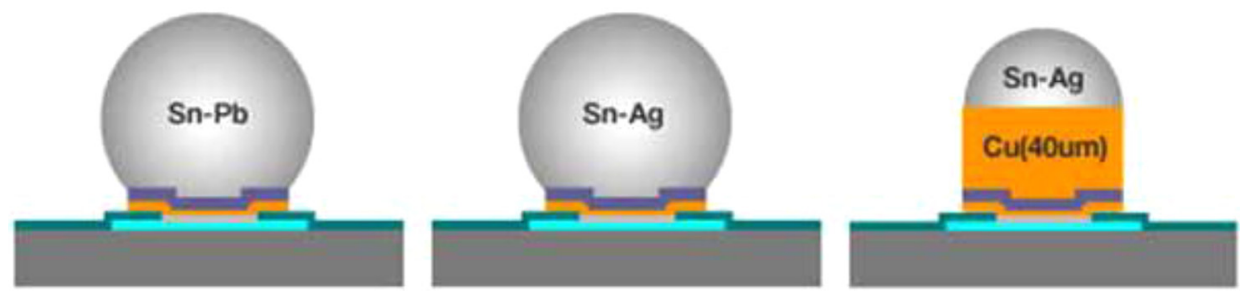

FIG. 20. Evolution map of $\mathrm{Cu}$ pillar bumps by Samsung. 
TABLE II. Scaling of chip-to-chip interconnect.

\begin{tabular}{|c|c|c|c|c|}
\hline Bonding Method & $\begin{array}{l}\text { C4 FC (Contolied } \\
\text { Collapse Chip } \\
\text { Connect) }\end{array}$ & $\begin{array}{l}\mathrm{C} 2 \mathrm{FC} \\
\text { (Chip Connect) }\end{array}$ & $\begin{array}{l}\text { TCIRR (Local } \\
\text { Reflow) FC }\end{array}$ & TC FC \\
\hline \multicolumn{5}{|l|}{ Schematic Diagram } \\
\hline $\begin{array}{l}\text { Major Bump Pitch Range at } \\
\text { Application }\end{array}$ & $>130 \mathrm{um}$ & $140 \mathrm{um} \sim 60 \mathrm{um}$ & $80 \mathrm{um}-20 \mathrm{um}$ & $<30$ um \\
\hline Bonding Method & $\begin{array}{l}\text { Conventional } \\
\text { Reflow }\end{array}$ & $\begin{array}{l}\text { Reflow with } \mathrm{Cu} \\
\text { pillar }\end{array}$ & $\begin{array}{l}\text { Thermal } \\
\text { Compression } \\
\text { with Cu pillar }\end{array}$ & $\begin{array}{l}\text { Thermal } \\
\text { Compression }\end{array}$ \\
\hline Bump Metallurgy & $\begin{array}{l}\text { Solder (SnAg or } \\
\text { SnAgCu) }\end{array}$ & $\begin{array}{l}\mathrm{Cu}+\text { Solder } \\
\text { (SnAg or Sn) }\end{array}$ & $\begin{array}{l}\mathrm{Cu}+\text { Solder } \\
\text { (SnAg or Sn) } \\
\text { Cap }\end{array}$ & $\mathrm{Cu}$ \\
\hline Bump Collapse & Yes & No & No & No \\
\hline Underfill Method & $\begin{array}{l}\text { - Capillary } \\
\text { - No flow }\end{array}$ & $\begin{array}{l}\text { - Capillary } \\
\text { - No flow } \\
\text { - Wafer Level }\end{array}$ & $\begin{array}{l}\text { - No flow } \\
\text { - Wafer Level }\end{array}$ & - Wafer Le \\
\hline
\end{tabular}

- A thin sputtered adhesion layer (e.g., Ti or TiW) covers the chip pad and overlaps onto the chip passivation.

- A sputtered conductive plating base $(\mathrm{Cu})$ is required as a 2nd layer for the subsequent deposition steps.

- A barrier layer (e.g., $\mathrm{Ni}$ or $\mathrm{Cu}$ ) is electroplated to prevent inter diffusion of solder and pad. In the case of a pillar bump, this layer is extended to a tall pillar of several $10 \mu \mathrm{m}$ height.

- Solder (e.g., lead-free SnAg) is plated on top of the barrier or pillar and reflowed to form a solder bump or cap.

In the case of a solder bump, the standoff between chip and substrate, on which the bumps are soldered, is determined by the controlled collapse of the large solder volume.
The surface tension of the molten solder keeps the chip at a distance during flip chip assembly. A solder mask around the substrate pads and the non-wettable chip passivation prevents the solder from spreading out indefinitely. One major benefit of collapsible bumps during reflow is that the formation of the flip chip bonds is very robust and highly tolerant to bump height variations. The pillar bumps, on the other hand, are more rigid and thus allow assembly with only a small solder volume on top of the pillars, which has two clear benefits, i.e., (1) the standoff can be kept high, even at very fine pitch, and (2) the solder will not spread out much on wettable surfaces, even without a solder mask barrier, due to the restricted solder volume. The main drawback of the
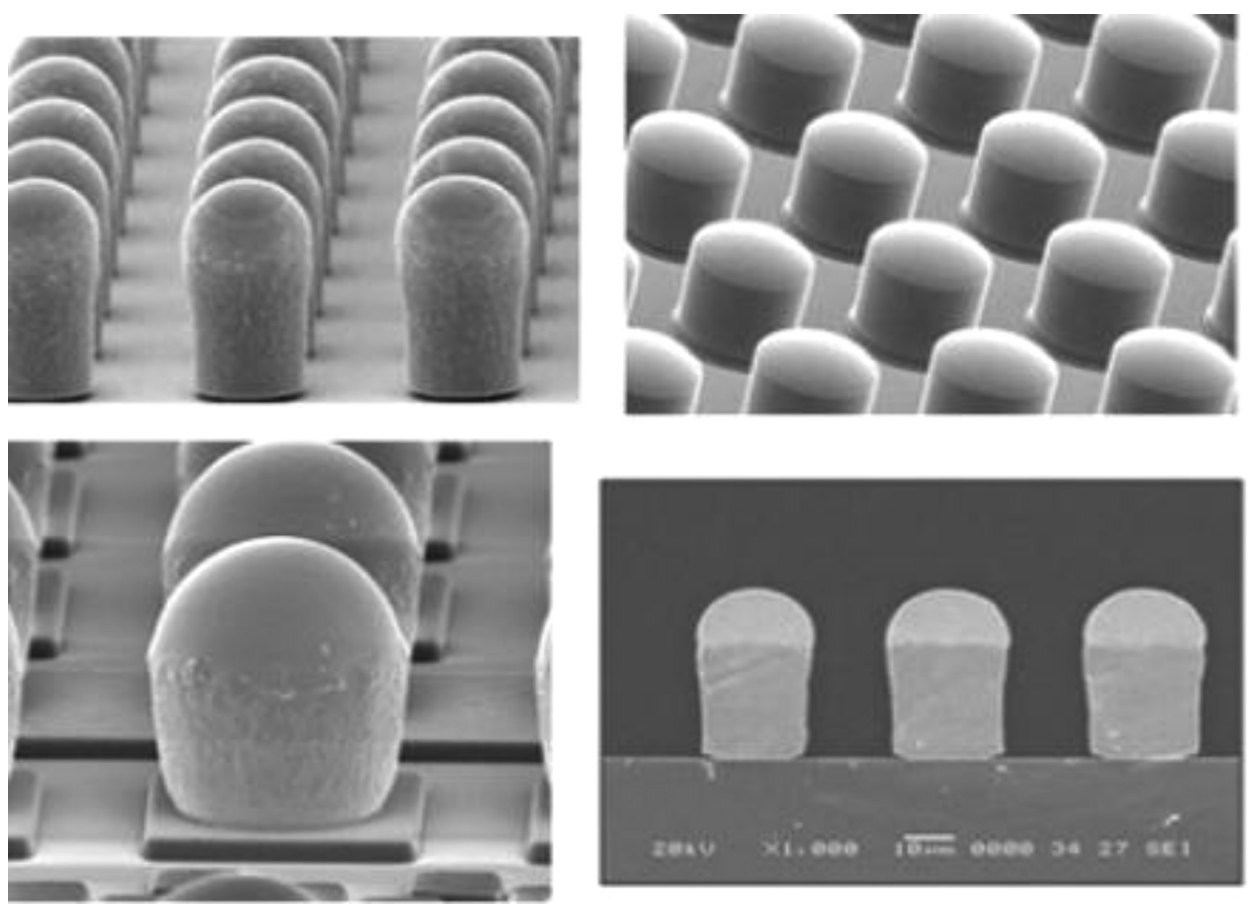

FIG. 21. SEM images of $\mathrm{Cu}$ pillar bumps. 


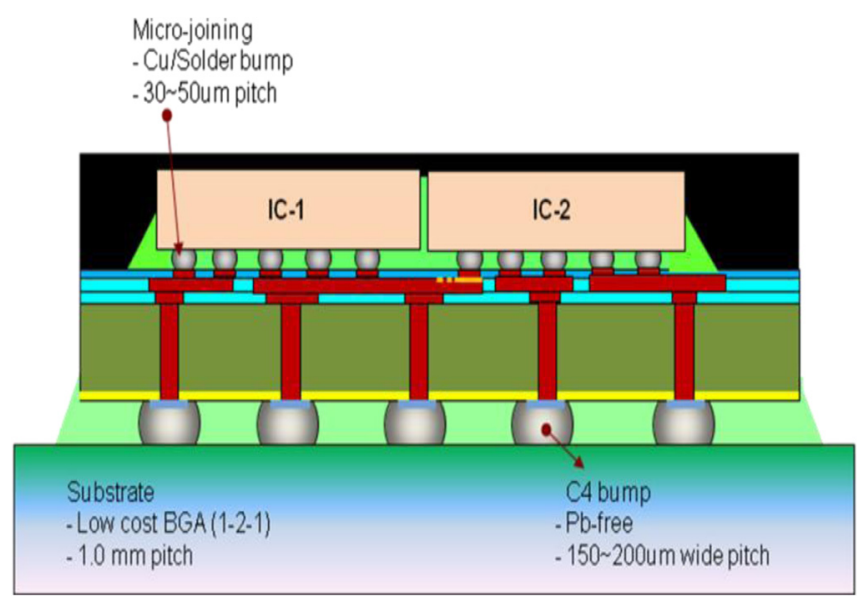

FIG. 22. Schematic of a typical 2.5D TSI system with two-die side-by-side integration.

reduced collapse of the small solder cap is that it requires tighter bump co-planarity control to prevent non-wetting of shorter bumps.

A tall $\mathrm{Cu}$ pillar offers additional advantages: thermal and electrical conductivity are higher than in a solder ball of similar size. Even more importantly, electro-migration (EM) reliability performance is much better in a $\mathrm{Cu}$ pillar where current crowding at the corner between chip and pad and bumps is located within higher EM-resistance bulk $\mathrm{Cu}$ instead of low-melting solder. A thin under bump metallurgy (UBM) in a solder bump cannot prevent high current densities at bump corner caused by current crowding. This leads to EM induced voids at the UBM/solder interface, which finally causes an open interconnect after high-current stress. The solder cap of the pillar bump makes it possible to use identical assembly process, assembly materials, and assembly equipment as for a solder bump of the same material.

$<50 \mu \mathrm{m}$ pitch $\mathrm{Cu}$ pillar bumps are reported by several organizations. ${ }^{63-71}$ IME reported their studies on fine-pitch $(40 \mu \mathrm{m}) \mathrm{Cu}$ pillar process integration and proposed a combined of wet and dry etching of seed layer removal method to reduce the $\mathrm{Cu}$ pillar undercut issue. ${ }^{66}$ IMEC reported on $10 \mu$ m electroplated CuSn micro-bumps with $20 \mu \mathrm{m}$ pitch in 2011. ${ }^{68,69}$ With further scaling down of $\mathrm{Cu}$ pillar bump diameter, seed layer etching was found to be a more and more important processing parameter to form finer pitch $\mathrm{Cu}$ pillar bumps. The seed etch process is compared between $\mathrm{Ti} / \mathrm{Cu}$ and TiW/Cu seed layers. During Ti etch, the etch rate dropped when the $\mathrm{Al}$ measurement pads are exposed. In order to remove the $\mathrm{Ti}$ layer between bumps to prevent shorts, the etch time needed to be increased, resulting in a non-uniform attach of $\mathrm{Al}$ measurement pads due to severe galvanic effect. With a TiW/Cu seed, no damage on the Al probing pads is observed. In addition, the $\mathrm{TiW} / \mathrm{Cu}$ based bumps has no undercut, while on the $\mathrm{Ti} / \mathrm{Cu}$ bump at each side more than $1 \mu \mathrm{m}$ undercut was observed. TiW was concluded as the preferred seed layer for fine-pitch $\mathrm{Cu}$ pillar bump. Based on the yield of the daisy chains, a defective density of the bumps below $50 \mathrm{ppm}$ is predicted. ITRI reported results on their $12 \mu \mathrm{m}$ micro-bumps $(5 \mu \mathrm{m} \mathrm{Cu} / 3 \mu \mathrm{m}$ $\mathrm{Ni} / 2.5 \mu \mathrm{m} \mathrm{SnAg}$ ) with $20 \mu \mathrm{m}$ pitch. ${ }^{70}$ Severe undercut caused by seed layer etching process is also found. Figure 23 shows the typical undercut of $\mathrm{Cu}$ pillar bump and the improved process for minimizing the undercut. ${ }^{70}$ Huang et al. reported that the reduction of $\mathrm{Cu}$ seed layer thickness from $5000 \AA$ to $2000 \AA$ and a dry etching process have been found to improve the undercut performance. ${ }^{70} \mathrm{Cu}$ pillar bumps with sub- $20 \mu \mathrm{m}$ pitch have also been reported by IME $(15 \mu \mathrm{m}$ pitch $)^{66}$ and Tohoku University $\left(10 \mu \mathrm{m}\right.$ pitch). ${ }^{67}$ For IME's $15 \mu \mathrm{m}$ pitch $\mathrm{Cu}$ pillar bumps, traditional electrochemical plating is used to deposit the $\mathrm{Cu}$ and $\mathrm{Sn}$ sequentially followed by photo resist stripping and seed layer etching which was carried out by a combination of wet $\mathrm{Cu}$ etching and dry Ta etching. Tohoku University reported an electroplatedevaporation bumping (EEB) technology to form $10 \mu \mathrm{m}$ pitch $\mathrm{Cu}$ pillar bumps, which is a combination of $\mathrm{Cu}$ electroplating and $\mathrm{Sn}$ evaporation. Resistance of $\mathrm{Cu} / \mathrm{Sn}$ bump was measured to be $35 \mathrm{~m} \Omega /$ bump.

\section{Reliability assessment of Cu pillar bumps}

Samsung did high temperature storage (HTS) test for $\mathrm{Cu}$ pillar bumps with $20 \mu \mathrm{m}$ in diameter and found that $\mathrm{Ni}_{3} \mathrm{Sn}_{4}$ intermetallic compound (IMC) formations at interface between SnAg solder and their $4 \mu \mathrm{m}$ Ni UBM degrade the mechanical properties of solder joint and increase resistance of solder bump. ${ }^{71}$ IMC growth rate and Ni UBM dissolution rate are calculated. The IMC changes into thick IMC during HTS as shown below in the SEM images. As shown in Figure $24,{ }^{71}$ thin IMC changes into thick IMC during High Storage Temperature (HST). During $150^{\circ} \mathrm{C}$ HST, Ni UBM is converted into $\mathrm{Ni}_{3} \mathrm{Sn}_{4} \mathrm{IMC}$, but resistance of the microbumps remains unchanged. Resistance started to degrade after $1000 \mathrm{~h}$ at $180^{\circ} \mathrm{C}$ storage due to void formation at interface between IMC and $\mathrm{Al}$ trace line, resulting in open failure upon complete consumption of Ni UBM. Figure 25 presents
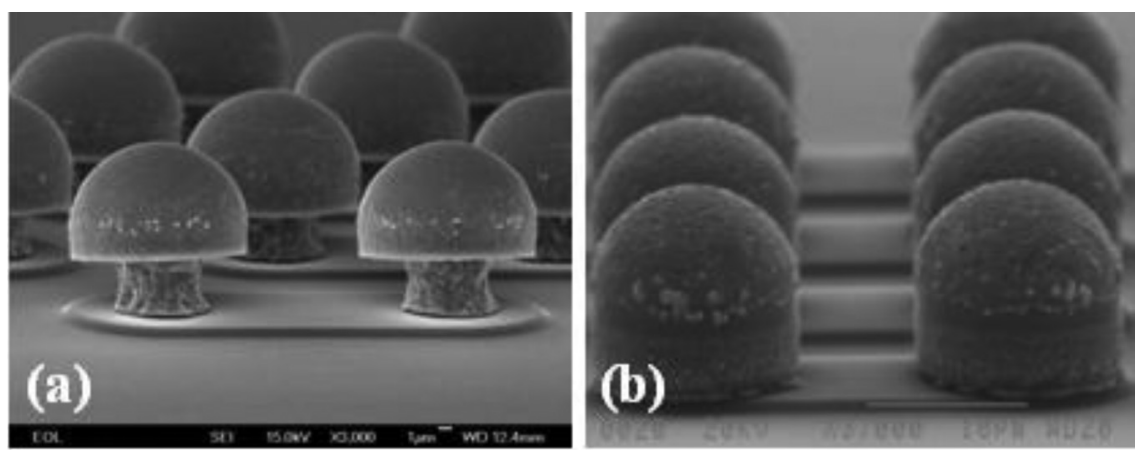

FIG. 23. $\mathrm{Cu}$ pillar bumps (a) with severe $\mathrm{Cu}$ post undercut and (b) fabricated with optimized process having minimized $\mathrm{Cu}$ post undercut. 

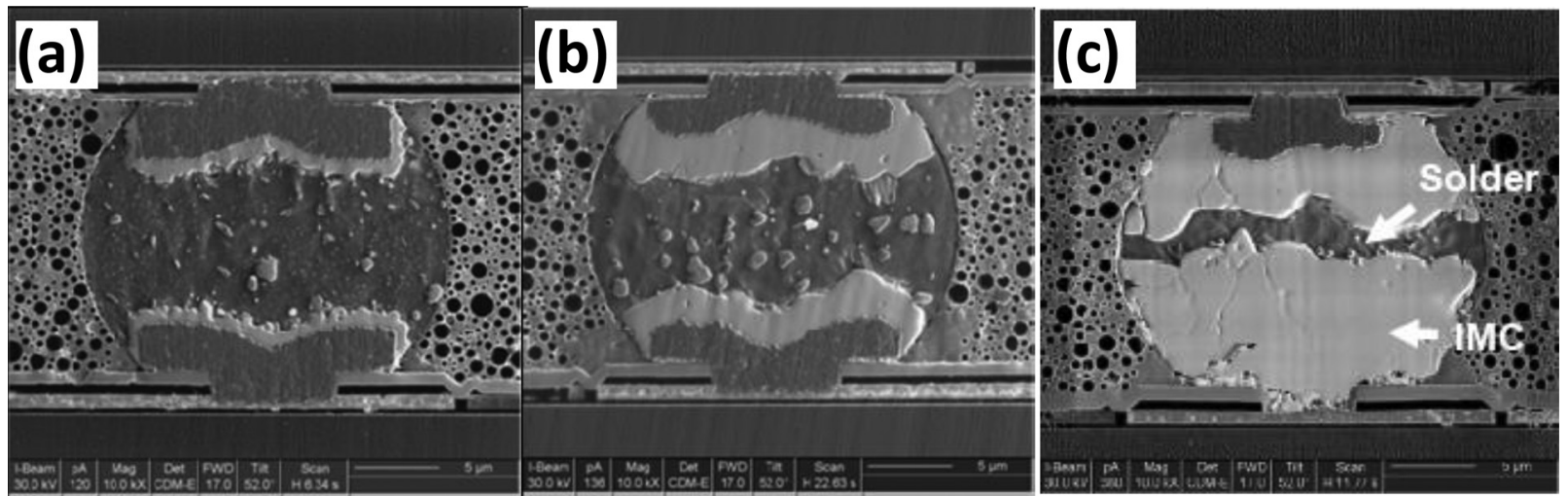

FIG. 24. SEM images of solder joints (a) as reflow condition, (b) $\mathrm{HTS} 150^{\circ} \mathrm{C}$ for $1300 \mathrm{~h}$, and (c) $180^{\circ} \mathrm{C}$ for $1300 \mathrm{~h}$.

the resistance evolution with time during $\mathrm{HTS}$ test at $150^{\circ} \mathrm{C}$ and $180^{\circ} \mathrm{C}$, respectively.

ITRI has also studied the failure mechanism of $20 \mu \mathrm{m}$ pitch using thermal cycling (TC) test. ${ }^{70}$ The IMC formed at the interface is identified as $\mathrm{Ni}_{3} \mathrm{Sn}_{4}$. The IMC thickness is also found to increase with time and/or temperature, which is consistent with the results reported by Samsung. Ni normally functions as a diffusion barrier to inhibit the reaction of $\mathrm{Sn}$ and $\mathrm{Cu}$ having very low solubility in Sn. During the bonding process, the bottom interposer is at $100^{\circ} \mathrm{C}$, and the top chip is at $280^{\circ} \mathrm{C}$ to melt $\mathrm{SnAg}$ solder alloy. It is concluded that the bonding temperature drives $\mathrm{Ni}$ atoms to dissolve into the melted solder and saturates the Sn matrix. As Ni has higher density than that of $\mathrm{Sn}$, gravity segregation occurs and causes the $\mathrm{Ni}$ atoms to accumulate at the interface and result in the thicker $\mathrm{Ni}_{3} \mathrm{Sn}_{4}$ formation. It is proposed that the failure mechanism of the micro-joints, as shown in Figure 26, is due to a Sn depletion zone which forms at the interface between Ni layer and Sn solder of the top chip due to the consumption of Sn. Eventually, the crack propagated along the interface and then the micro-joint fails when stress induced by Z-axial expansion of underfill is exerted on the interface.

$\mathrm{EM}$ is the migration of metal in the direction of electron flow which results in void formation at the electrons entrance (or current exit, I-) and metal pile-up at the electrons exit (or current entrance, $\mathrm{I}+$ ). Since void formation is the most detrimental effect of EM, the current exit I- is the most critical interface. While there have been many EM studies of $100 \mu \mathrm{m}$ and larger bumps, there are not many studies on fine pitch $\mathrm{Cu}$ pillar bumps, especially below $20 \mu \mathrm{m}$ size. It was found that with good Ni barrier structures, CuSn bumps may have EM lifetimes similar to or greater than eutectic PbSn bumps. ${ }^{54,61}$ Samsung reported EM accelerated life time assessment for $\mathrm{Cu}$ pillar bumps. Figure 27 shows the microstructure of microbumps with $6.4 \times 10^{4} \mathrm{~A} / \mathrm{cm}^{2}$ current stressing and without current stressing for $624 \mathrm{~h}$ at $150{ }^{\circ} \mathrm{C}$. $^{71}$ In Figure 27, polarity effect was observed. During current stressing, the migration direction of Ni is the same as the electron flow, so Ni UBM at cathode side was consumed. Also migrated Ni from cathode to anode enhances IMC growth at anode. It is calculated based on memory device application conditions of $105^{\circ} \mathrm{C}$. The EM life time of $\mathrm{Cu}$ pillar bump with Ni UBM achieved more than 100 years based on $0.1 \%$ EM failure, as shown in Figure 28.

IMEC has studied on the EM performance comparison between solder flip chip bumps (thin layer of Ni/Au UBM and a SAC solder) and $\mathrm{Cu}$ pillar bumps $(40 \mu \mathrm{m} \mathrm{Cu}$ pillar with $20 \mu \mathrm{m} \mathrm{SnAg} \mathrm{cap)} \mathrm{with} \mathrm{same} \mathrm{diameter,} \mathrm{i.e.,} 60 \mu \mathrm{m} .{ }^{72} \mathrm{~A}$ clear difference in EM behavior was observed between standard $\mathrm{NiAu} / \mathrm{SAC}$ and $\mathrm{Cu}$ pillar bumps. Figure 29 shows SEM images of solder bumps under thermal stress without and with EM stress for both standard solder bump and $\mathrm{Cu}$ pillar bump. ${ }^{72}$ Standard bumps with Ni/Au UBM show a consistent failure mechanism of micro-structural degradation through void formation at the interface of solder and IMC, and this occurs for all test conditions used $\left(150-170^{\circ} \mathrm{C}\right.$ and $300-500 \mathrm{~mA}$ ). However, $\mathrm{Cu}$ pillar bumps did not show any electrical, nor micro-structural degradation. It was concluded that less current crowding for $\mathrm{Cu}$ pillar bumps was expected that that of solder bumps, which causes less local EM damage. The other reason of the outstanding EM performance of $\mathrm{Cu}$ pillar bumps is its faster formation of a full intermetallic phase. As soon as all solder materials is transferred into
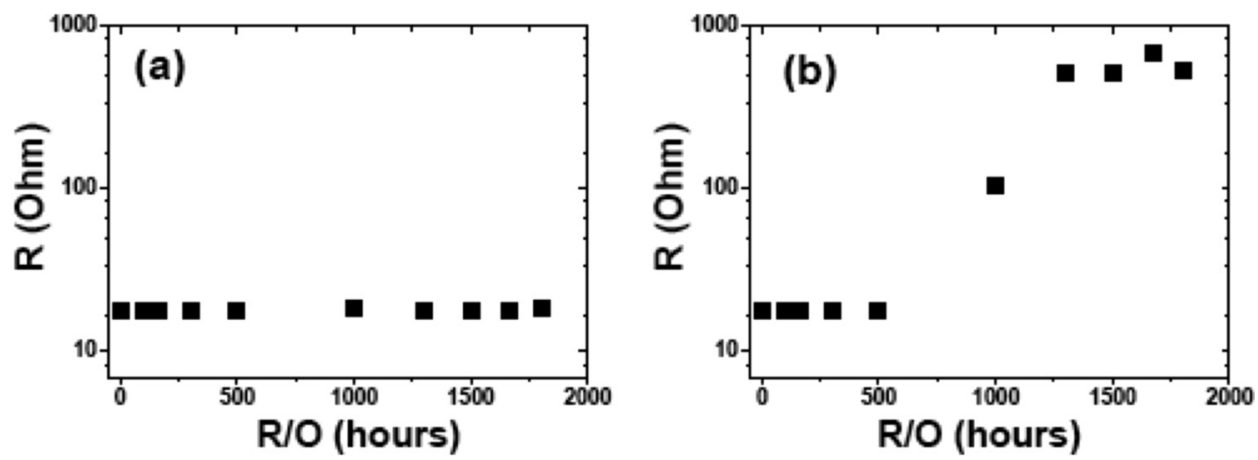

FIG. 25. Resistance as a function of storage time during HTS test at (a) $150{ }^{\circ} \mathrm{C}$ and (b) $180^{\circ} \mathrm{C}$. 


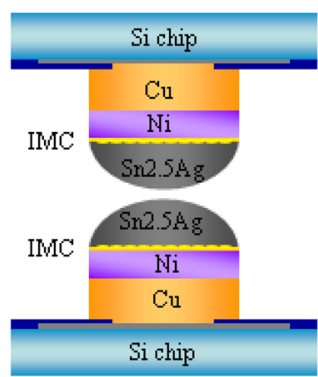

(a) Before bonding

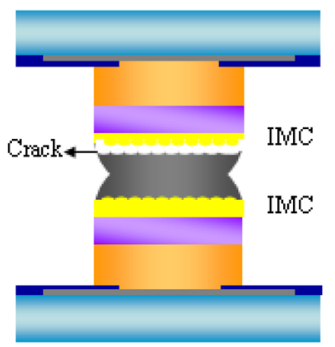

(d) After TCT 3000 cycles

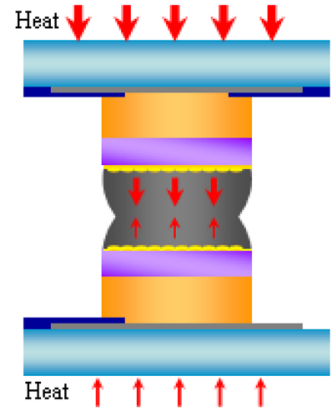

(b) Bonding

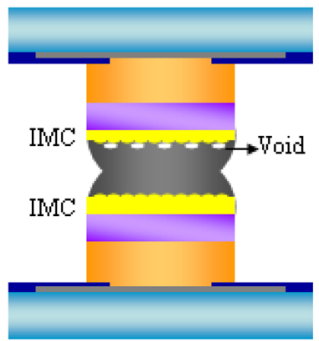

(c) After bonding / After TCT 100 cycles
FIG. 26. Schematic diagrams of the failure mechanism of micro-joint. (a) Before bonding, (b) bonding, (c) after bonding/after TCT 100 cycles, and (d) after TCT 3000 cycles.

intermetallic phase, the joint is no longer expected to fail at reasonable testing conditions which are compatible with packaging materials.

\section{Thin wafer handling}

TSVs are continuously being scaled down for various benefits. These benefits include higher I/O densities, smaller form factor, reduced process cost, and smaller keep-out-zones (KOZ) for logic devices. TSV dimensions indirectly determine the thickness of an interposer. However, as TSV dimensions are scaled downwards (in particular TSV depth), wafer handling becomes an issue. For wafer thickness below 100 $\mu \mathrm{m}$, warpage becomes an issue without any support. As such, the device wafer would have to be temporary supported on tape or on a carrier wafer by means of adhesives. ${ }^{73,74}$ Figures 30 and 31 illustrate a typical bonding and debonding process, respectively.

In summary, thin-wafer handling can be simplified into different categories based on (1) type of carrier and handling wafer and (2) bonding and debonding mechanisms. ${ }^{52,53}$

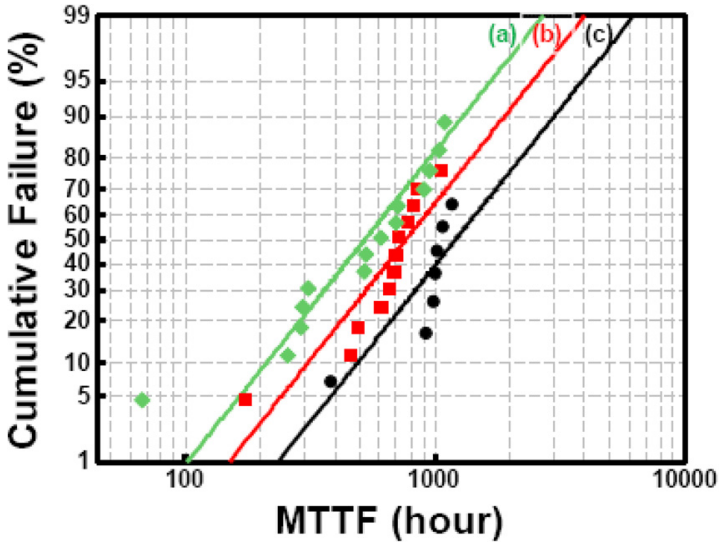

FIG. 28. Log-normal distribution of EM failure at $150^{\circ} \mathrm{C}$ under various current densities of (a) $6.4 \times 10^{4} \mathrm{~A} / \mathrm{cm}^{2}$, (b) $5.6 \times 10^{4} \mathrm{~A} / \mathrm{cm}^{2}$, and (c) $5.1 \times 10^{4} \mathrm{~A} / \mathrm{cm}^{2}$.

\section{Carrier substrates}

The substrate used is highly dependent on the temporary bonding/debonding systems used. Si carrier wafer is preferred in most cases when light source or laser is not needed to be shined onto the wafer. For cases where solvent is needed to pass through the carrier, perforations are needed; for cases where light needs to pass through the carrier wafer for debonding, glass substrates will need to be used.

\section{Temporary bonding and debonding}

As illustrated in Figure 32, current temporary bonding methods encompass mechanical peel-off, thermal slide, solvent and laser (light) release. ${ }^{73,74}$

Mechanical release or peel-off systems make use of adhesives that are bonded and debonded at room temperature. This process is very dependent on the adhesives used. The adhesive should have enough strength to hold the bonded wafers together during grinding and processing but still allow the bonded wafer to be peeled apart. Thermal slide process, on the other hand, uses a combination of heat and pressure to bond the wafers together. The maximum sustainable process temperature and the debonding temperature are limited by the bonding temperature. Si carrier wafer is mainly used for both mechanical and thermal slide-off process and have good total thickness variation (TTV). Solvent release involves the use of a carrier wafer with perforations, which serves to allow solvent to get in touch with the adhesives that bonds the wafer to the perforated carrier wafer.
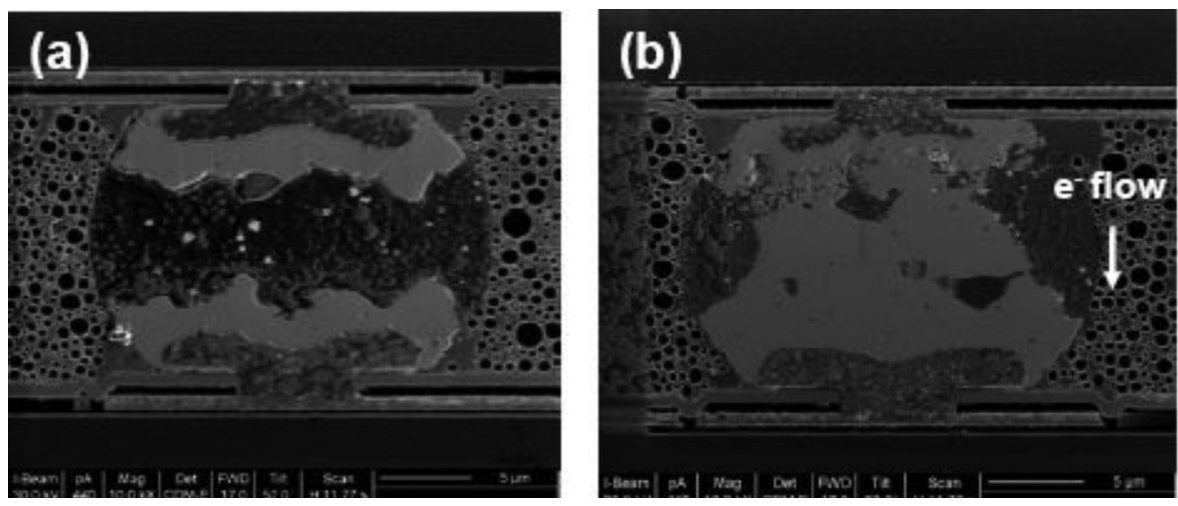

FIG. 27. SEM images of solder bump (a) without current stressing for $624 \mathrm{~h}$ and (b) with EM current stressing of $6.4 \times 10^{4} \mathrm{~A} / \mathrm{cm}^{2}$ for $624 \mathrm{~h}$ at $150{ }^{\circ} \mathrm{C}$. 


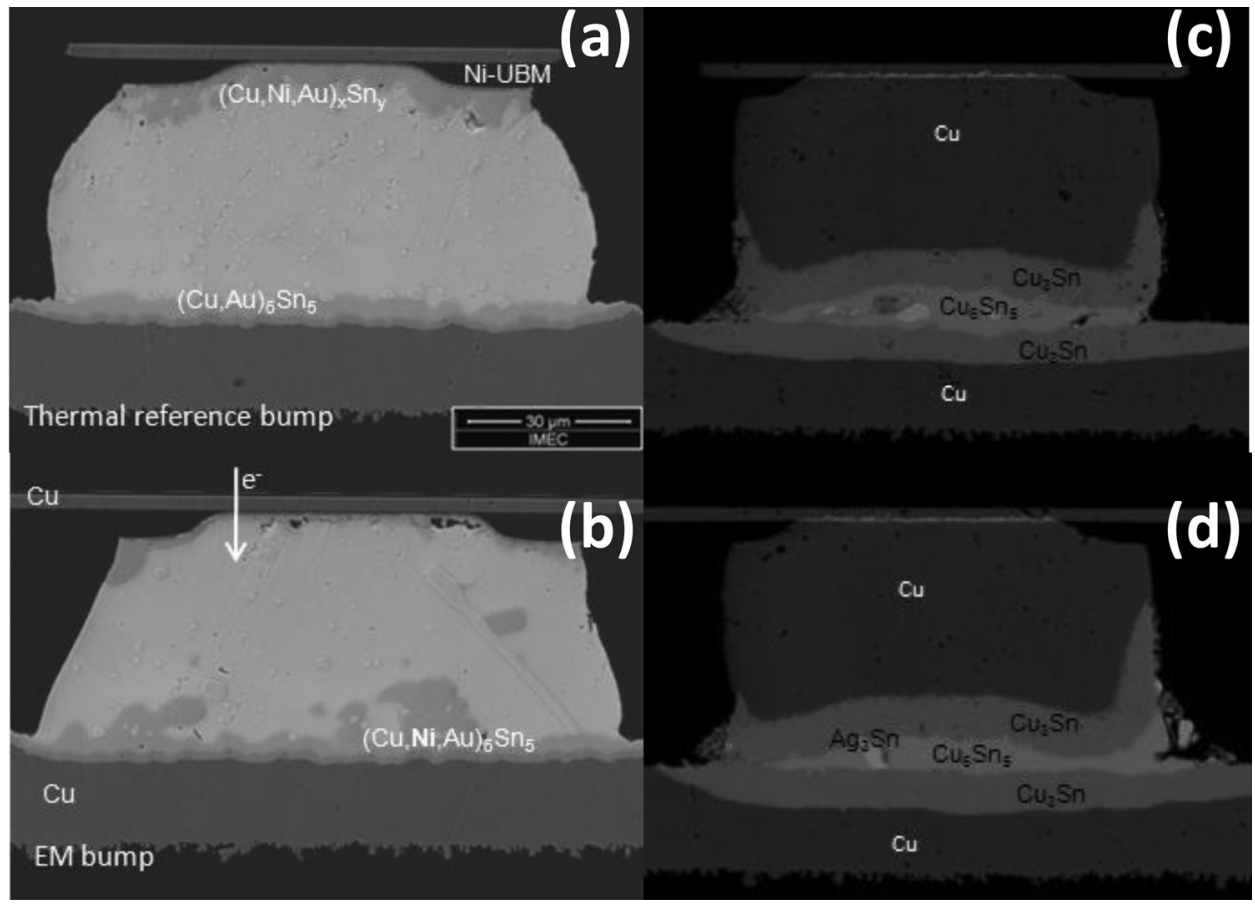

FIG. 29. Cross-sectional images of bumps after EM testing. (a) Thermal reference solder bump at $150^{\circ} \mathrm{C}$ without EM stress, (b) solder bumps at $170^{\circ} \mathrm{C}$ with EM stress of $500 \mathrm{~mA}$, (c) thermal reference $\mathrm{Cu}$ pillar bump at $170^{\circ} \mathrm{C}$ without EM stress, and (d) $\mathrm{Cu}$ pillar bump at $170^{\circ} \mathrm{C}$ with EM stress of 300 $\mathrm{mA}^{72}$ Reproduced with permission from Labie et al., "Outperformance of $\mathrm{Cu}$ pillar flip chip bumps in electromigration testing," in Proceedings of the 61st Electronic Components and Technology Conference (ECTC), Lake Buena Vista, FL, USA (2011), p. 312.

Copyright 2011 Institute of Electrical and Electronics Engineers.

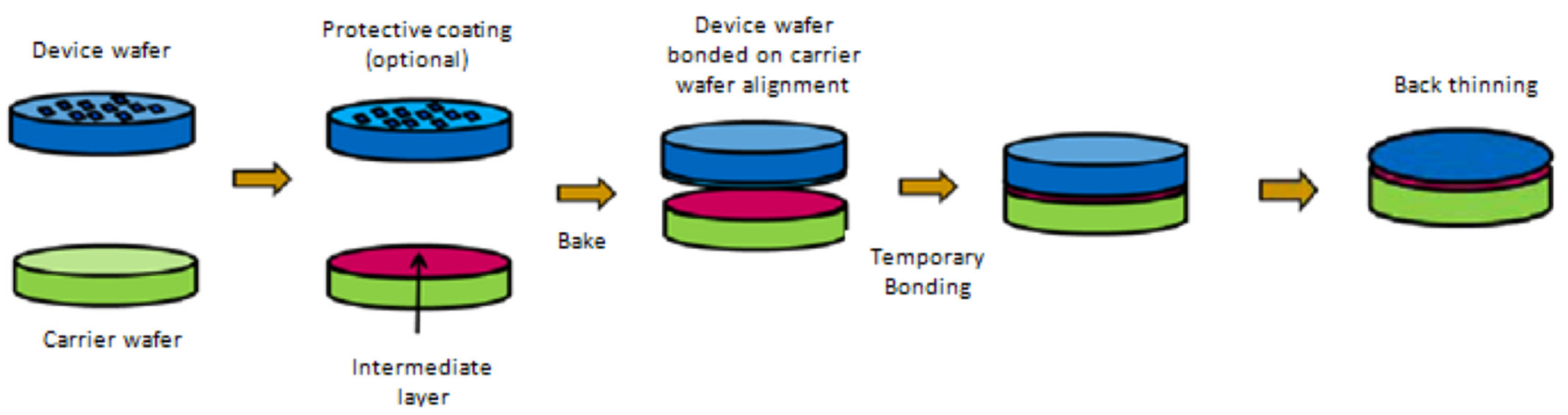

FIG. 30. A typical temporary bonding process flow.

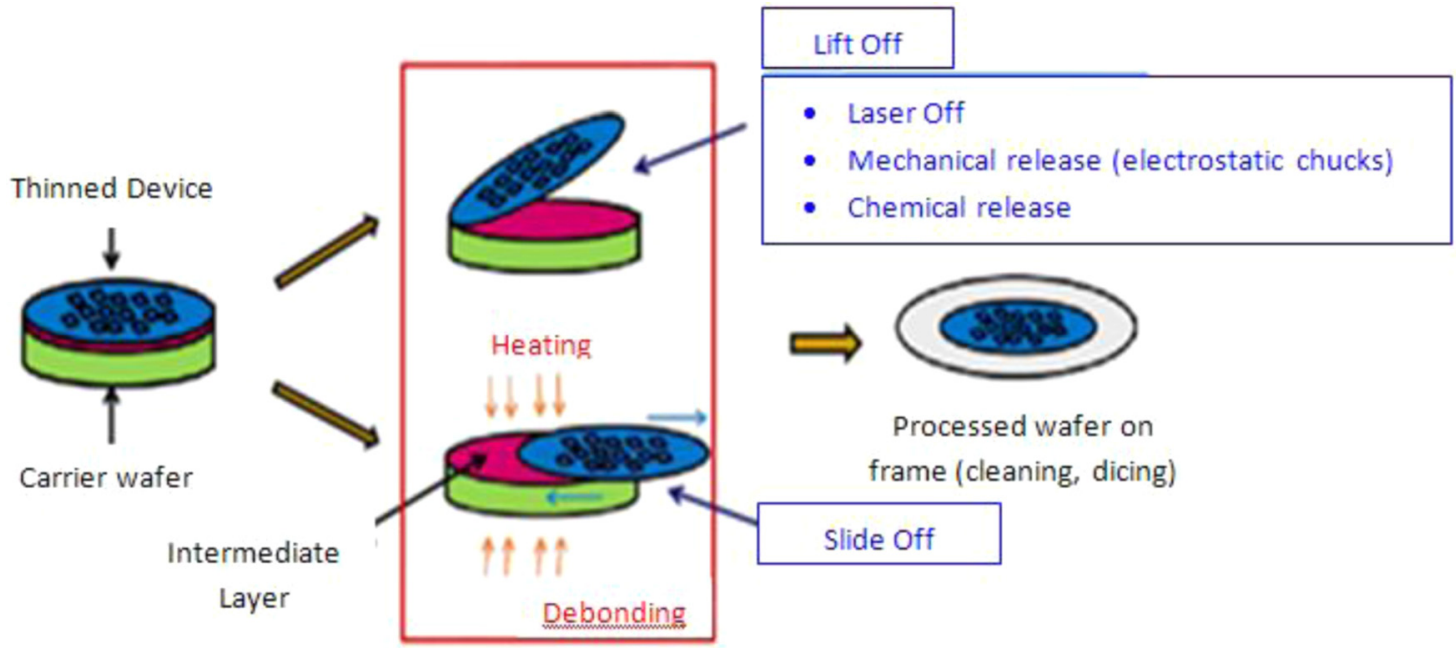

FIG. 31. A typical debonding process flow. 


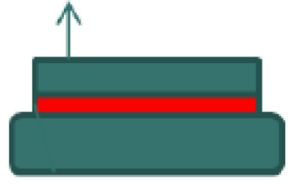
Mechanical

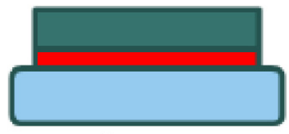

Laser

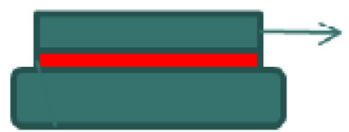

Thermal slide

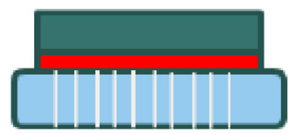

Solvent
FIG. 32. Temporary bonding and debonding processes.

However, contamination of tool chucks can be a possible issue. In addition, the holes in the wafer can get dirty or clogged over use with time. Lastly, in laser release process, laser/light sensitive adhesives are used for bonding. Laserbonded wafers are basically debonded by passing laser or light through the backside of the carrier wafer. This approach is thermally stable, however, only glass substrates can be used. Table III shows a summary of the various temporary bonding and debonding systems.

\section{Adhesives requirement}

High temperature resistance, chemical resistance, high mechanical strength, low TTV capability, low out-gassing, easy and clean removal of adhesives, and costs are some of the key requirements of temporary bonding and debonding adhesives. Depending on the use and application, not all of these requirements are critical. The selection of requirements, the respective adhesives, and bonding systems is very dependent on the user application and subsequent processes.

\section{2.5D TSI ASSEMBLY CHALLENGES}

\section{A. Introduction}

A typical 2.5D interposer package consists of one or multiple guest dies stacked on top of a thinned TSV interposer, which is in turn assembled on either an organic flip chip BGA (FCBGA) or a ceramic substrate. ${ }^{75-79}$ Figure 33 shows an example of a $2.5 \mathrm{D}$ interposer package. The two guest chips could be assembled side-by-side on the interposer as shown in the schematics or be replaced by one or more stacks of multiple dies (for example, memory cube). By using the 3D stacking of multiple chips, the total Si area that can be housed in a single package is significantly increased. It also dramatically improves the device performance due to the extremely short interconnect for between die communication. In this section, assembly flow and challenges of 2.5D TSI package are reviewed.

\section{B. TSI package assembly process flow}

There are 3 basic 2.5D TSI assembly flow options: chipon-chip first (CoC-first), chip-on-substrate (CoS-first), and chip-on-wafer first (CoW-first). For each of the flip chip attach process, there are choices of using mass reflow or thermal compression bonding (TCB) to form the solder joints between two tiers of chips or substrate. Upon the completion of the flip chip solder joint connection, underfill is applied to encapsulate the gap between the two tiers of the chip or substrate for the purpose of pro-longing the solder joints fatigue life and creep performance. The underfill also serves as environmental protection agent to avoid solder corrosion. There are several underfill process flow options as well. ${ }^{80-83}$ The most commonly used underfill methods for TSI assembly are non-conductive paste (NCP) and capillary underfill (CUF). The NCP is applied during flip chip attach and can only be used in conjunction with TCB assembly, while the CUF is used after the flip chip attach is done and can be used in conjunction with both TCB and mass reflow chip attach processes. Figure 34 shows examples of the block diagrams of two commonly used process flows for CoS-first and CoC-first assembly. The text descriptions of each process flow and the pros and cons are in Secs. III B 1 and III B 2. The CoW-first assembly flow integrates the TSV wafer processing with guest chip attach before completion of the TSI wafer fabrication and is known as chip-on-wafer-onsubstrate $(\mathrm{CoWoS}){ }^{77-79}$ Section III B 3 describes the CoWoS process and an example of the assembled package.

TABLE III. Summary of carrier wafer and corresponding wafer handling methodologies.

Carrier type TBDB mechanisms Disadvantages Advantages

Silicon canter

Glass carrier

(1)Thermal slide-off

(2) Solvent release

(3) Mechanical release

(1) UV/laser release
(1) Compatible wish semi eqpt

(2) Available with good TTV

(3) Low cost

(1) Can check wafer after each process

(2) Can use UV/laser release
(1) Edge trimming for carrier wafer

(2) Temperature limited

(3) Cannot use UV/laser debondling process

(1) Higher cost to achieve good TTV

(2) CTE not matched with Si

(3) Easier to break

(4) Not compatible with ESC (cannot try mechanical release)

(1) Perforated holes degrade TTV

(2) Higher cost

(3) Not compatible with ESC (cannot try mechanical release)
Carrierless

No need debonding
(1) No carrier needed. No TBDB

(1) Non-standard post processing required. Not compatible with some semi process tools

(2) Need special grinder 


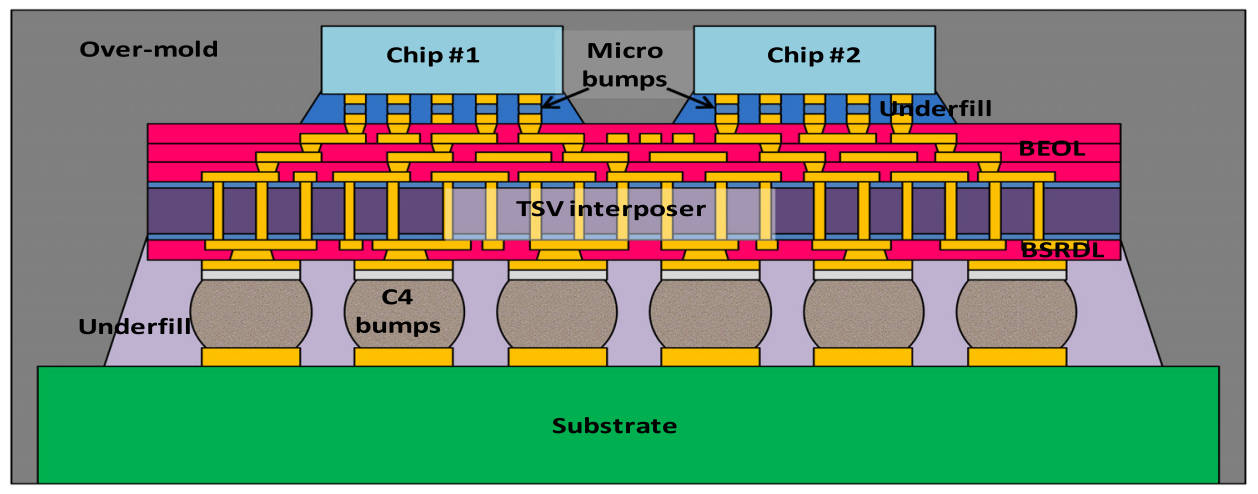

FIG. 33. Schematics of 2.5D package with two guest dies assembled side-byside on top of TSV interposer.

\section{Chip-on-substrate (CoS) first assembly flow}

In this process flow, the TSI die is flip-chip attached onto the substrate $(\mathrm{CoS})$ first. The chip attach can be done by either a mass reflow plus CUF or by TCB plus NCP process. Once the $\mathrm{CoS}$ is completed, the guest dies are assembled onto the CoS stack by TCB plus NCP process to complete the process for final test. The advantage of this assembly flow is that it allows testing of the KGD (known good dies) TSI before committing to the expensive functional guest dies and avoids wasting of costly functional dies. The disadvantage of this assembly flow is that the tier 2 assembly of guest dies onto the $\mathrm{CoS}$ could be challenging if the $\mathrm{CoS}$ (i.e., TSI on Substrate) has large warpage due to the difference in CTE between $\mathrm{Si} \quad(\mathrm{CTE} \sim 3 \mathrm{ppm})$ and organic substrate (CTE $\sim 16 \mathrm{ppm}$ ) which is further amplified for large size TSI die. A highly warped CoS could hinder successful microbump connection between the guest dies and the TSI. It also could hinder underfill flow to successfully fill the gap between the guest dies and the TSI. The usage of ceramic
CoC-First

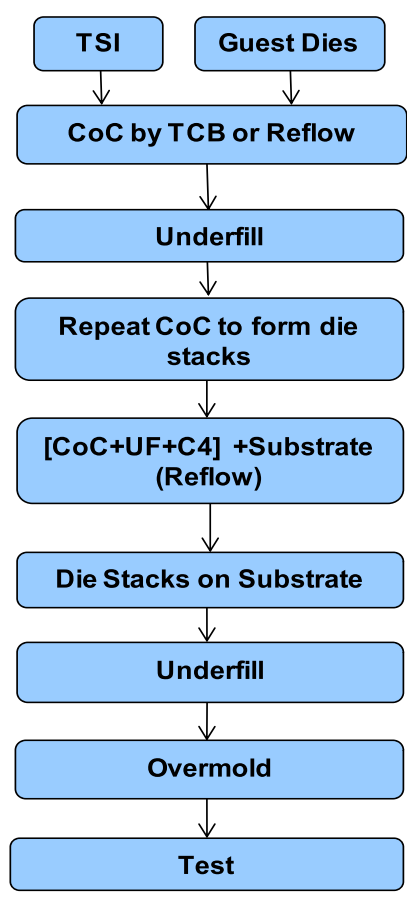

CoS-First

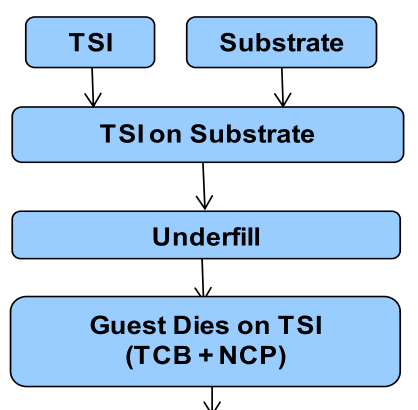

$\downarrow$

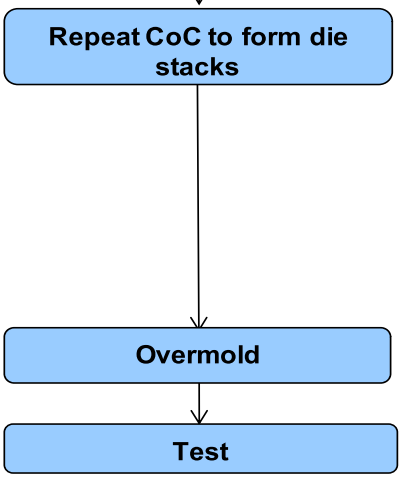

FIG. 34. Assembly flow options for TSI packaging. substrate which has similar CTE with Si chip can be used to mitigate the warpage issue.

\section{Chip-on-chip (CoC) first assembly flow}

In this process flow, the guest dies are flip chip attached onto the TSI chip (CoC) first. The chip attach is done by one of these three process flows: (\#1) mass reflow plus CUF, (\#2) TCB plus CUF, or (\#3) TCB plus NCP process. Once the $\mathrm{CoC}$ assembly is completed, the $\mathrm{CoC}$ die stack is assembled onto the substrate by mass reflow plus CUF to complete the assembly for final test. While it is easier to control the micro-bump assembly in the CoC-first approach than the CoS-first approach due to smaller virgin-TSI die warpage than assembled $\mathrm{CoS}$ stack warpage, the handling of the $\mathrm{CoC}$ stack for next tier assembly (i.e., assembly of $\mathrm{CoC}$ onto substrate) and post-CoC stack warpage has to be managed for a successful assembly of the CoC onto substrate. Figure 35(a) shows a severely warped $\mathrm{CoC}$ die stack failed to make full contact with substrate bondpad after flux tacking and before solder reflow. However, the solder joints were successfully formed between the CoC TSI and the substrate after solder reflow (Figure 35(b)), although the solder joints were stretched at edges of the TSI. The biggest disadvantage of this $\mathrm{CoC}$-first approach is that it is not possible to test the post-CoC joints for a KGD selection to avoid potential waste of expensive functional dies if the TSI itself or the CoC assembly is not good.

\section{Chip-on-wafer (CoW) first assembly flow}

In this CoW-first assembly flow, ${ }^{77-79}$ the guest dies are flip-chip bonded on TSI wafer after the completion of TSV formation, front-side multi-layer interconnect, and front-side micro-bump formation, but before back-side wafer thinning and TSV reveal during the TSI wafer fabrication. After the completion of guest chips attach, the CoW wafer is molded and thinned down to $100 \mu \mathrm{m}$ or less from the backside to reveal the TSV and is followed by $\mathrm{C} 4$ bumping process before it is singulated into individual "CoW" dies for assembly onto substrate to complete the CoWoS process flow. This CoW-first assembly flow is heavily promoted by TSMC and is called $\mathrm{CoWoS}^{77-79}$ technology. The schematics of the CoWoS process flow are shown in Figure 36. Examples of a TSV wafer which is populated with guest dies (CoW) and an assembled TSI package are shown in Figure $37 .^{77}$ The CoWfirst flow is favorable for micro-bump joining due to the low 

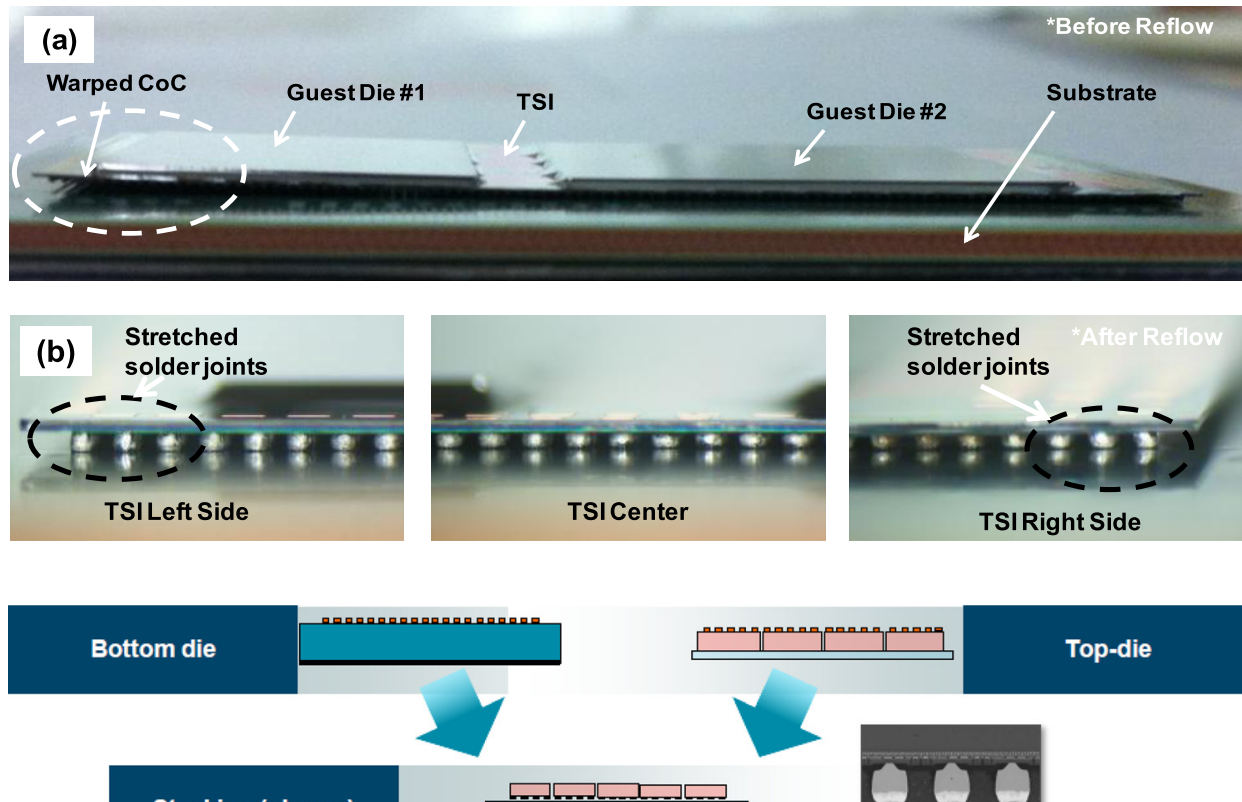

Stacking ( $\mu$ bump)
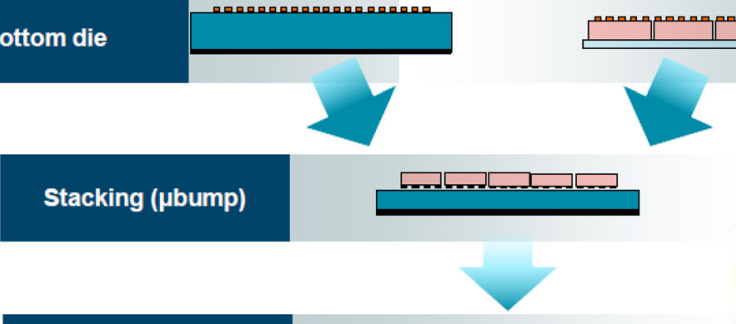

Wafer Molding
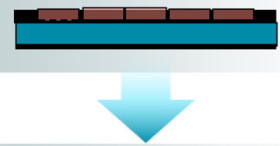

Carrier bonding

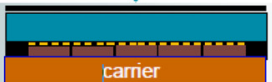

B/S grinding
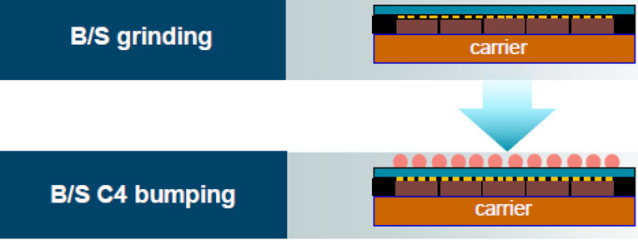

\section{Transfer glass to tape}

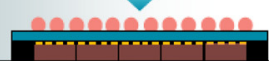
Tape

\section{Singulation}

mpirm mperm Tape

\section{TIS (Stacking, C4)}

Top-die
Build up Subs.

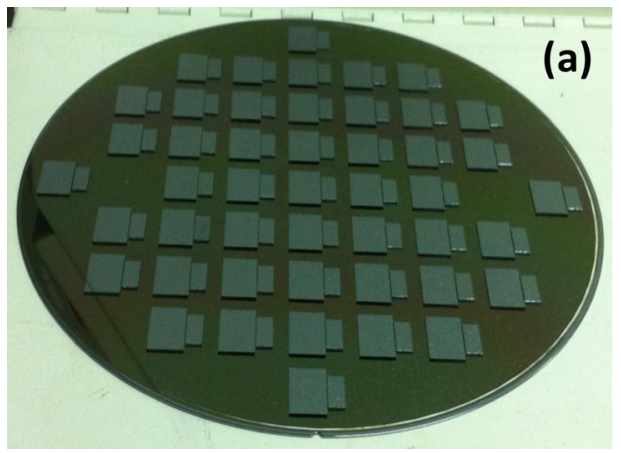

FIG. 35. CoC-First assembly: (a) before reflow and (b) after reflow (Courtesy of Institute of Microelectronics)
FIG. 36. CoWoS process flow.

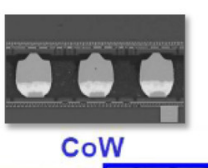

EIG. 36. CowoS process flow.

FIG. 37. (a) The top view of chip-onwafer and (b) chip-on-TSI-on-substrate.

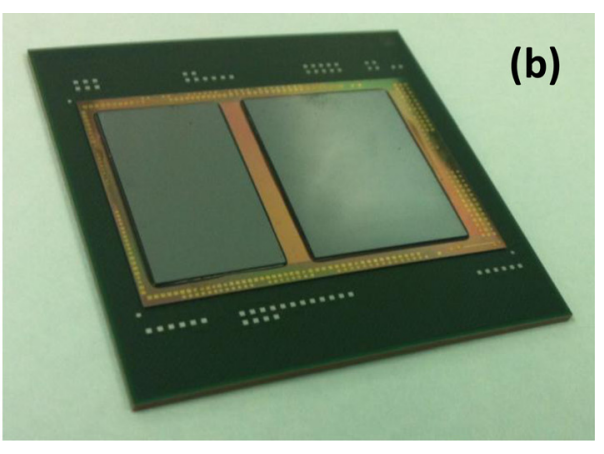


warpage of the full thickness $\mathrm{Si}$ wafer, and no need of thin wafer handling during die attach process. However, the wafer level warpage becomes quite severe after the CoW wafer is overmolded with epoxy mold compound for the downstream processing and may require expensive investment in tools for stringent inline warpage control.

\section{Assembly Challenges and Warpage Control}

Although all the basic steps to assemble 2.5D TSI package (flip chip attach, solder reflow, thermo-compression bonding, underfill, over-mold encapsulation, etc.) are available in the traditional flip chip packaging technology, the integration of these steps coupled with the requirements of ultra-thin die (100 $\mu \mathrm{m}$ and below), super-fine pitch $(50 \mu \mathrm{m}$ and below), small underfill gap (20 $\mu \mathrm{m}$ and below), and three-dimensional stacking have made the $2.5 \mathrm{D} / 3 \mathrm{D}$ assembly very challenging for not only materials selection and process development but also assembly tool capability of handling highly warped/stressed and fragile wafer, die, and package. For a successful 2.5D TSI assembly, the multiple levels of interaction among Si chip, bump interconnect, underfill, substrate, and mold compound has to be considered starting with the package physical design/routing and materials selection, the assembly flow sequence selection (CoC-first or CoS-first, or CoW-first), and the assembly tool capability to handle such high level of complex integration.

The warpage issue arises from the CTE mismatch between Si and substrate. By adding underfill between these two components to enhance solder joint reliability and encapsulating the device and substrate with epoxy mold compound to protect the package can make the package warpage either worse or better, depending on the mechanical and thermo-mechanical properties of the underfill and mold compound, and the relative thickness of each components. There is no one-size-fits-all remedy for this challenge. Use modeling and simulation to predict the warpage, especially the warpage at assembly temperature, is a very useful and economic way to help select a suitable assembly process flow for a given package design and material set to minimize the process development time.

Figure 38 is a schematic illustration of the warpage related assembly issues. Three assembly issues can be easily seen from this schematic: (a) Bumps merged at the area
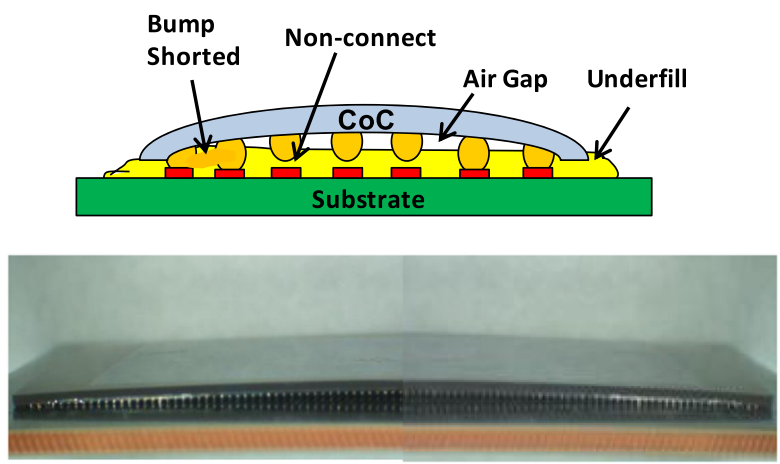

FIG. 38. Warpage related assembly issues: Bump shorted, bump nonconnect, and underfill with air gap under the chip (Photo: Courtesy of Institute of Microelectronics). where the space between bumps become small, (b) bumps not connected due to extra distance between bump and substrate bondpad, and (c) underfill fails to fill the gap between chip and substrate due to extra space/height. The relationship of process flow sequence and warpage is experimentally demonstrated by Murayama et al..$^{84}$ In this study, the test vehicle consists of two guest dies of $10 \times 10 \times 0.75 \mathrm{~mm}$ size and $50 \mu \mathrm{m}$ size micro-bumps; a TSI interposer of $26 \mathrm{~mm}$ $\times 26 \mathrm{~mm} \times 0.1 \mathrm{~mm}$ size, $10 \mu \mathrm{m} / 50 \mu \mathrm{m}$ diameter/pitch TSV, 2 metal layers on top and 1 metal layer on bottom side of TSI; and an organic substrate of $40 \mathrm{~mm} \times 40 \mathrm{~mm} \times 1 \mathrm{~mm}$ size and $0.8 \mathrm{~mm}$ thick core and with $\mathrm{Sn}-57 \mathrm{Bi}$ low melting point solder. The resulting warpage profile of the TSI assembly is shown in Figure 39, where it shows the Si-IP + Substrate (i.e., CoS-first) has the worst warpage and is the most challenge for the subsequent guest dies assembly onto this $\mathrm{CoS}$ stack. It also shows a significant increase of warpage when the $\mathrm{CoC}$ stack is assembly onto the substrate, although it is not as bad as the CoS-first approach. This study also concluded that by using low $\mathrm{Tg}$ and high CTE underfill for $\mathrm{CoC}$ assembly, and by using low melting temperature solder for the $\mathrm{CoS}$ assembly, the warpage can be minimized.

Several other methods have been proposed and demonstrated to have benefits of mitigating the 2.5D TSI assembly warpage issues. Examples are: using ceramic substrate, ${ }^{85}$ using TCB for flip chip attach, ${ }^{86}$ and using specially design pick up tool. ${ }^{87}$ The effect of underfill and warpage on package stress and reliability is also been reported..$^{88-90}$

\section{Summary}

The 2.5D TSI packaging is a highly integrated system. Although the basic building block is no difference than the traditional flip chip packaging, the multiple tiers of chip, solder, underfill, and substrate stacking and with miniaturization of all physical dimensions inside the package make the assembly of such package extremely challenging. Materials and processing integration alone can no longer satisfy the demand for successful assembly and reliability of the $2.5 \mathrm{D}$

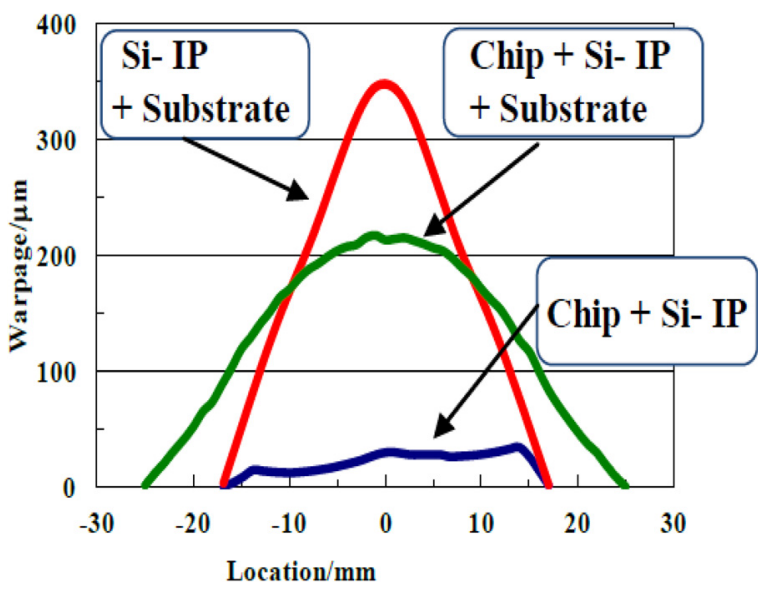

FIG. 39. Comparison of warpage after bonding. ${ }^{84}$ Reproduced with permission from Murayama et al., "Warpage control of silicon interposer for 2.5D package application," in Proceedings of the IEEE-Electronic Components \& Technology Conference (ECTC) (2013), p. 879. Copyright 2013 Institute of Electrical and Electronics Engineers. 


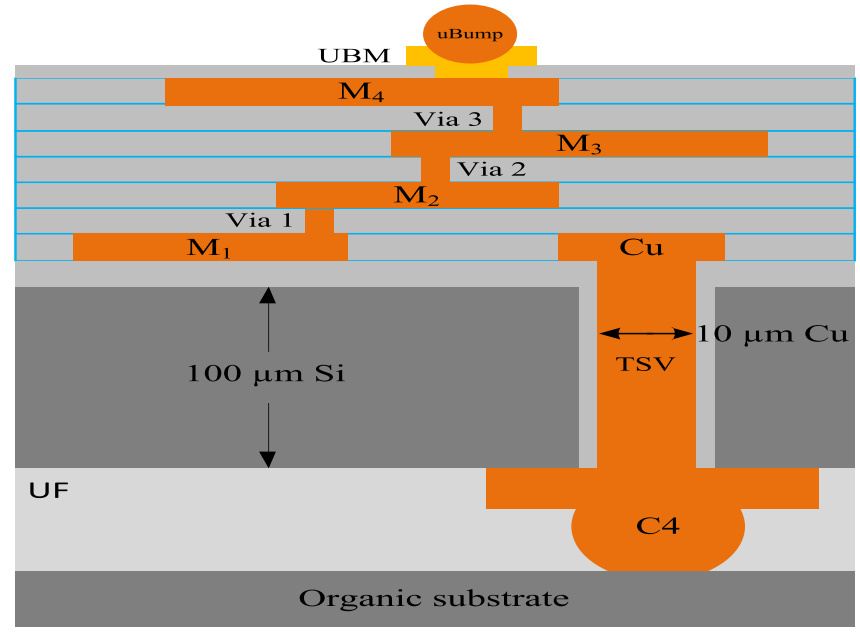

FIG. 40. Cross section view of through Si interposer.

packaging. A new paradigm of packaging integration must start with the TSI design for low stress, low warpage TSI as the foundation for the 2.5D assembly, take into consideration of various process flow sequence and the impacts of interactions among the package materials, including the Si device, the substrate, and everything between them.

\section{2.5D TSI TEST AND CHARACTERIZATION}

Si verified 2.5D TSI PDK ${ }^{91}$ is mandatory for the design engineers to achieve first time design success for their high speed/low power 2.5D TSI circuits and systems. At the core of Si verified 2.5D PDK lie a suite of test structures ${ }^{92}$ aimed at characterizing the individual components and parasitic elements of 2.5D TSI technology. The objective of test structures is two-fold: (a) To provide an electrical data that can be used to verify electrical models to be incorporated into $2.5 \mathrm{D}$ TSI PDK and (b) to enable the debugging of $2.5 \mathrm{D}$ process development. This section illustrates various test structures aimed at characterizing individual 2.5D TSI components and parasitic elements followed by characterization results to be incorporated into Si verified PDK.

\section{A. Test structures for 2.5D TSI electrical characterization}

Cross-section of a typical passive $\mathrm{Si}$ interposer with 4 BEOL metals at the front sides fabricated with $\mathrm{Cu}$ damascene process, and one thick backside RDL processed after TSV revealing followed by back side micro-bump attachment is illustrated in Figure 40. The key technology components and parasitic elements include front-side microbumps, front-side BEOL interconnects, TSVs, backside RDL, and back side micro-bumps. To characterize these components, the test structures (Figure 41) consist of: (a) Metal meander-folks for measuring sheet resistance and intra-layer capacitance of every metal layer, (b) four-port Kelvin structure for via and TSV/BEOL via resistance measurement, (c) Daisy chains to check connectivity between different levels of BEOL metals individually and collectively, (d) exclusive M1 - TSV - Back-side RDL - TSV M1 daisy chains to characterize TSV resistance and debug the TSV and back side reveal process, (e) exclusive M4 front-side micro-bump - guest die M1 - front-side microbump - M4 daisy chains to characterize Front side microbump resistance ensuring the connectivity of guest die to the 2.5D TSI, (f) Exclusive back-side RDL - back-side microbump - substrate trace - back-side micro-bump - back-side RDL to characterize back-side micro-bump resistance and connectivity between 2.5D TSI and substrate, (g) parallel plate capacitances for measuring inter-metal capacitance, (h) embedded and de-embedded TSV banks for TSV and backside micro-bump capacitance characterization, and (i) transmission lines to characterize time domain (eye diagram) and frequency domain (s-parameter) high-speed signal transmission responses across the interposer.

Parametric analyzer set-up is used for resistance measurements while LCR meter is employed for capacitance characterization. RF characterization is performed with network analyzer. With the help of standard resistance and capacitance models, ${ }^{93}$ it is ensured that the measureable resistance of the test structure is $\gtrsim 1 \Omega$ and capacitance is $\gtrsim 100 \mathrm{fF}$ unless it is a very a sensitive measurement of a single TSV through four-port Kelvin structure. Typical number of vias/ front-side micro-bumps and back-side micro-bumps in a typical daisy chain is between $\sim 90$ and 1000 such that the same chains can be used for reliability assessment as well. Few characterization results are summarized in Sec. IV A 1 and IV A 2.

\section{TSV characterization structures}

To accurately characterize the TSV resistance, four-port Kelvin structure is commonly used to measure a single TSV as shown in Figure 41(b). Alternatively, a series of TSVs as a daisy-chain in Figure 41(c) is used as well. With interposer thickness of $100 \mu \mathrm{m}$, the TSV diameter is $10 \mu \mathrm{m}$ (aspect ratio of 1:10). The measured TSV resistance is shown in Figure 42. Its mean value across three wafers is measured to be $23 \mathrm{~m} \Omega$ matching well with the modeled TSV resistance $21.4 \mathrm{~m} \Omega$ plus metal contact resistance between TSV and wafer metal layers. (The metal layers are in series with the TSV under test.)

Charge-Based-Capacitance-Measurement $(\mathrm{CBCM})^{94}$ is a possible solution for measuring single-TSV capacitance. However, such technique uses active circuits, which is not suitable for the passive interposer. TSV embedded and de-embedded structures illustrated in Figure 41(h) are used to characterize TSV capacitance as well as the back-side micro-bump capacitance. Measured TSV capacitance is subtracted from the TSV + back-side micro-bump capacitance and is normalized to obtain the back-side micro-bump capacitance which is estimated to be $\sim 120 \mathrm{fF}$. Similarly, we measured de-embedded structure capacitance without any TSVs to capture the BEOL metal capacitance. It is subtracted from the embedded TSV capacitance and is normalized to extract the nominal TSV capacitance. C-V measurements performed at $10 \mathrm{kHz}-1 \mathrm{Mhz},-40$ to $40 \mathrm{~V}$ gate voltage on TSV-to-Si substrate capacitance structure using the thick Si substrate acting as an ohmic substrate contact are demonstrated in Figure 43. In this case, TSV behaves as a metal-oxide-semiconductor (MOS) capacitor 


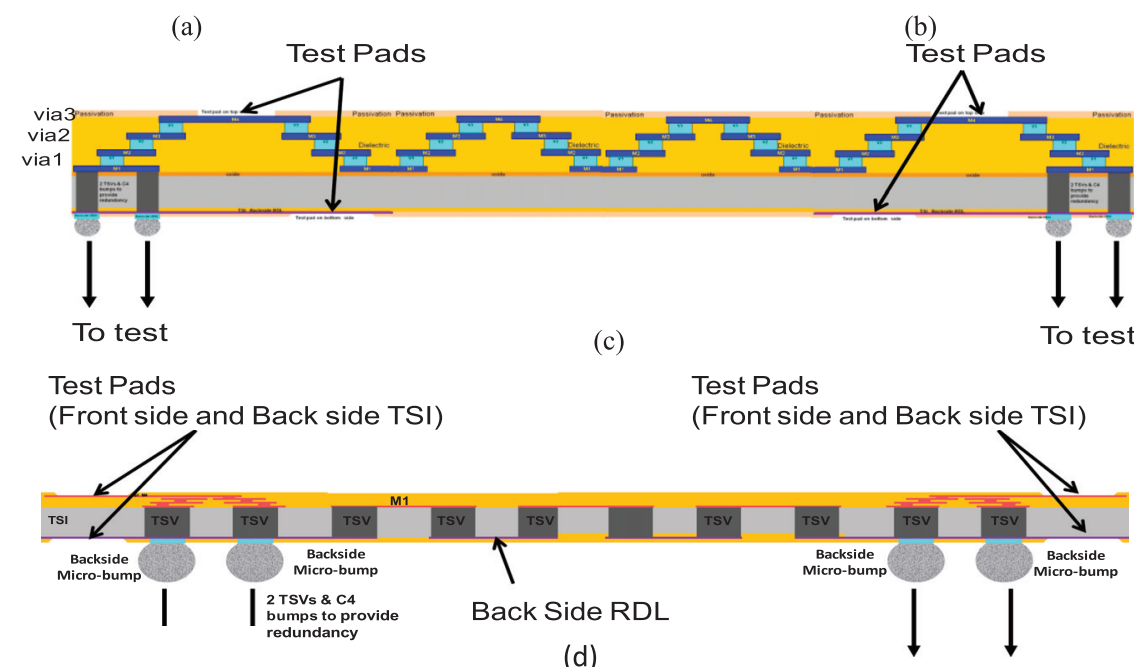

To BGA substrate \& test jig

To BGA substrate \& test jig

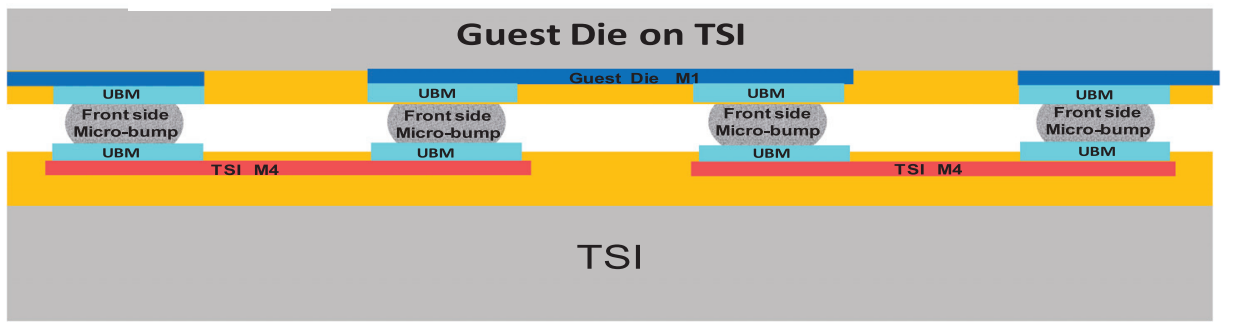

(e)

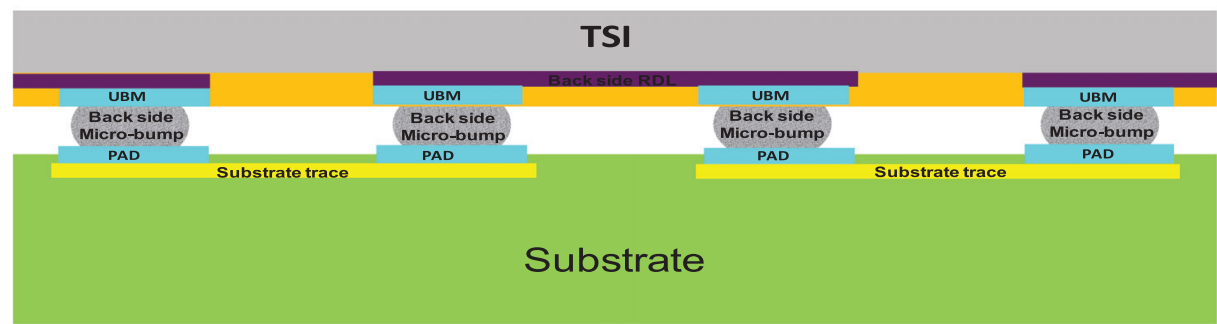

(f)

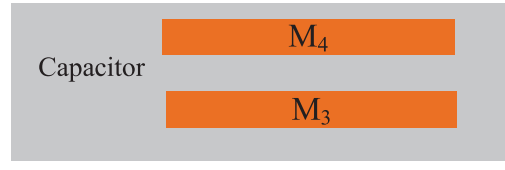

(g)

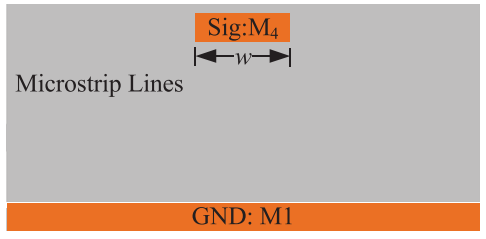

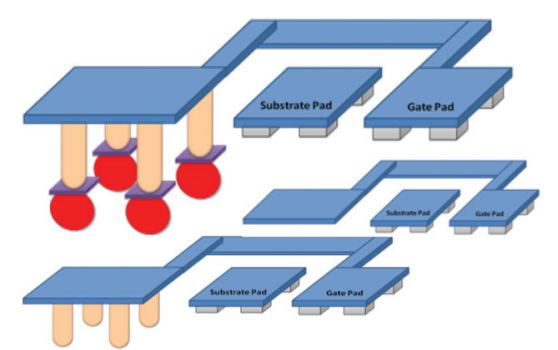

(h)

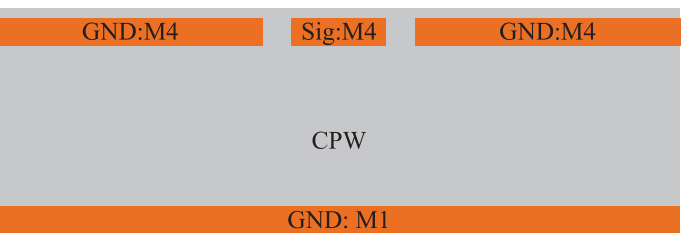

(i)

FIG. 41. TSI test structures: (a) meander fork structure, (b) four-port Kelvin structure for TSV/BEOL via resistance measurement, (c) Daisy chain for BEOL characterization, (d) exclusive daisy chain for TSV characterization, (e) exclusive daisy chain for Front-side micro-bump characterization, (f) exclusive daisy chain for Back-side micro-bump characterization, (g) parallel plate capacitor, (h) embedded and de-embedded TSV banks for TSV and C4 bump characterization, and (i) transmission line structure, micro strip (left) and CPW (right). 


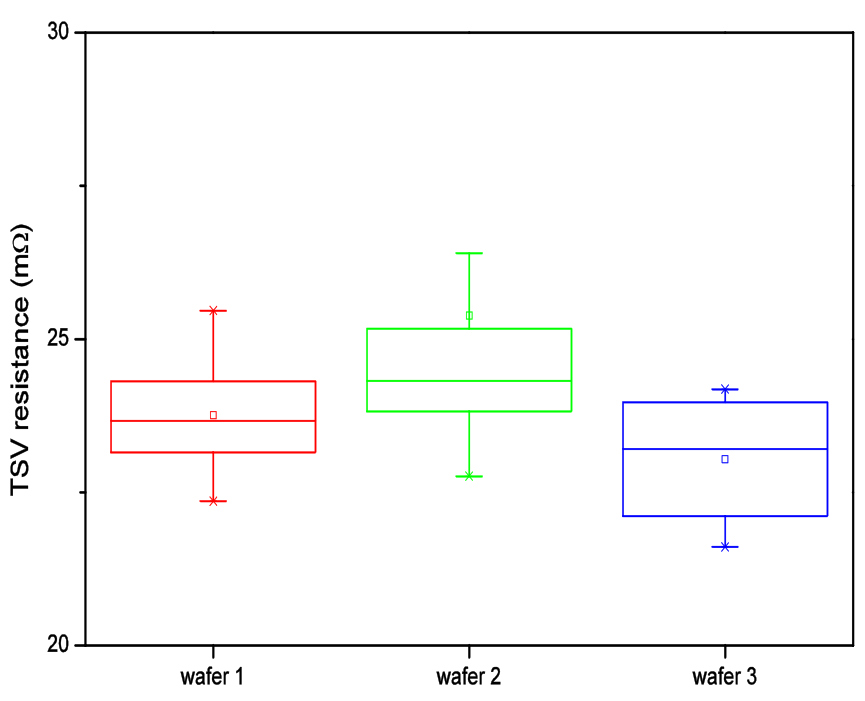

FIG. 42. Measurement results of TSV resistance.

demonstrating the accumulation, depletion, maximum depletion (high frequency $\sim 1 \mathrm{MHz}$ ) or inversion (low frequency $\sim 10 \mathrm{kHz}$ ) capacitance behavior as the voltage is sweeped between -tive and + tive gate voltages. The measured $\mathrm{C}-\mathrm{V}$ curve along with the TSV analytical capacitance model $^{95}$ is used to extract key TSV parameters listed in Table IV. TSV FIB images confirm their dimensions as $12 \mu \mathrm{m}$ outer diameter and $100 \mu \mathrm{m}$ height. TSV oxide capacitance in the accumulation is measured to be $300 \mathrm{fF}$, while the capacitance in the maximum depletion region is $180 \mathrm{fF}$. Using TSV dimensions of diameter $=12 \mu \mathrm{m}$ and height $=100 \mu \mathrm{m}$, the equivalent oxide liner thickness is extracted to be $\sim 420 \mathrm{~nm}$. The measured negative threshold voltage $=-1 \mathrm{~V}$ is attributed to the extracted higher fixed oxide charges $\mathrm{Q}_{\mathrm{f}}=1.3 \times 10^{11} / \mathrm{cm}^{2}$ and lower $\mathrm{D}_{\mathrm{it}}=4.12 \times 10^{10} /$ $\mathrm{cm}^{2}-\mathrm{eV}$.

\section{High density wiring characterization}

Key aspects of the electrical models as described above are resistance, capacitance, and eye-diagram measurements. Meander-fork structures shown in Figure 41(a) are used to characterize interconnects' sheet resistance and intra-layer capacitance. For $1 \mu \mathrm{m}$ thick M1 to $\mathrm{M} 4 \mathrm{Cu}$ lines having width $=2 \mu \mathrm{m}$ and spacing $=2 \mu \mathrm{m}$, the measured sheet

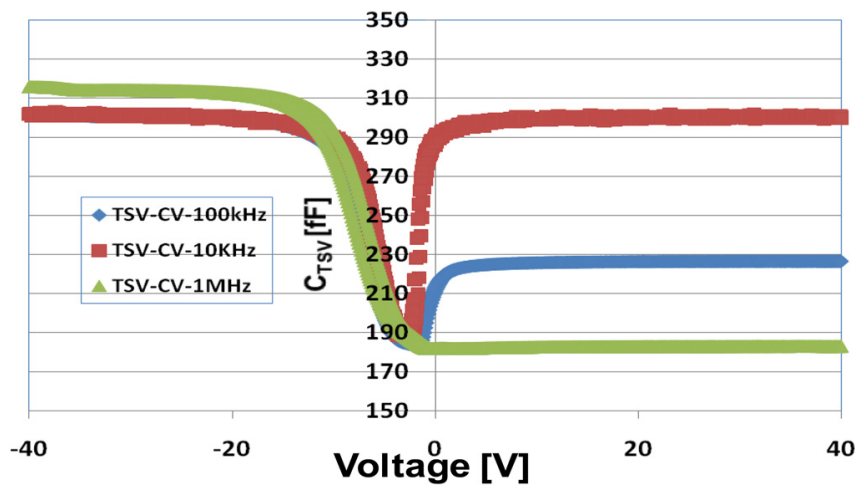

FIG. 43. Measured TSV capacitance result with different bias voltage and frequency.
TABLE IV. Extracted parameters of TSV in TSI.

\begin{tabular}{lcc}
\hline \hline & $\begin{array}{c}\text { Physical } \\
\text { specifications/estimations }\end{array}$ & \\
\hline$\phi_{\mathrm{TSV}}(\mu \mathrm{m})$ & 12 & Measured \\
$\mathrm{L}_{\mathrm{TSV}}(\mu \mathrm{m})$ & 100 & Measured \\
$\mathrm{C}_{\mathrm{ox}}(\mathrm{fF})$ & 300 & Measured \\
$\mathrm{C}_{\mathrm{TSV}}(\mathrm{fF})($ Ohmic Contact $)$ & 180 & Measured \\
$\mathrm{V}_{\mathrm{th}}(\mathrm{V})$ & -1 & Extracted \\
Average oxide liner & 420 & \\
thickness $(\mathrm{nm})$ & $8 \times 10^{14}$ & Extracted \\
$\mathrm{N}_{\mathrm{A}}\left(/ \mathrm{cm}^{3}\right)$ & $1.3 \times 10^{11}$ & Extracted \\
$\mathrm{Q}_{\mathrm{f}}\left(/ \mathrm{cm}^{2}\right)$ & $4.12 \times 10^{10}$ & Extracted \\
$\mathrm{D}_{\mathrm{it}}\left(/ \mathrm{cm}^{2}-\mathrm{eV}\right)$ & $2.14 \times 10^{10}$ & Extracted \\
$\mathrm{Q}_{\mathrm{m}}\left(/ \mathrm{cm}^{2}\right)$ & & \\
\hline \hline
\end{tabular}

resistance and intra-layer capacitance across 5 wafers are illustrated in Figures 44(a) and 44(b) accordingly. The mean sheet-resistance and capacitance is $14.2 \mathrm{~m} \Omega / \square$ and 34.1 $\mathrm{fF} / \mathrm{mm}$, respectively. In our $2.5 \mathrm{D}$ TSI designs, we typically use 4-10 $\mu \mathrm{m}$ wide interconnects to achieve lower resistance and short "resistance-capacitance (RC)" delay. By using thicker copper traces, such as $3 \mu \mathrm{m}$ RDL technology, one can achieve lower resistance of $5.7 \mathrm{~m} \Omega / \square$, which leads to higher data rate due to less parasitics. Large parallel-plate capacitors as shown in Figure 41(g) are used to characterize

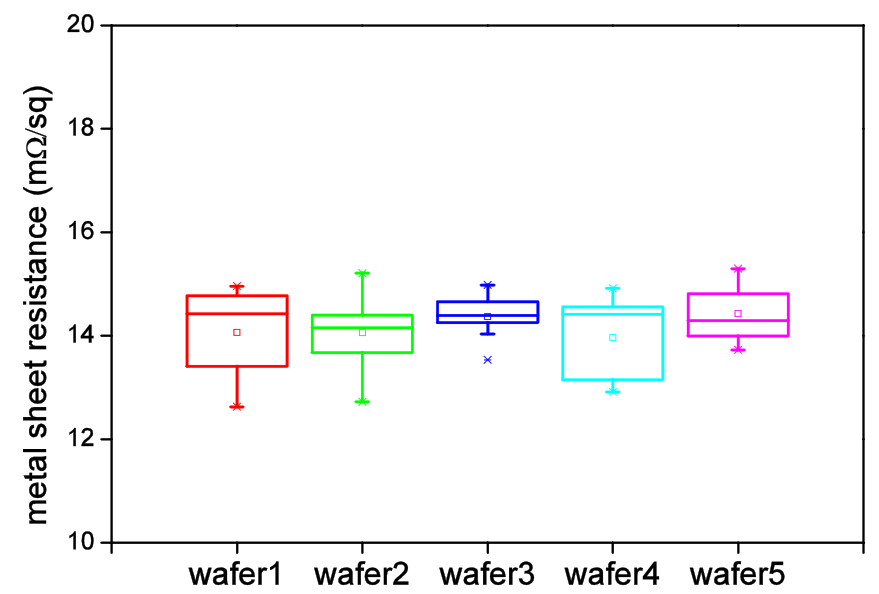

(a)

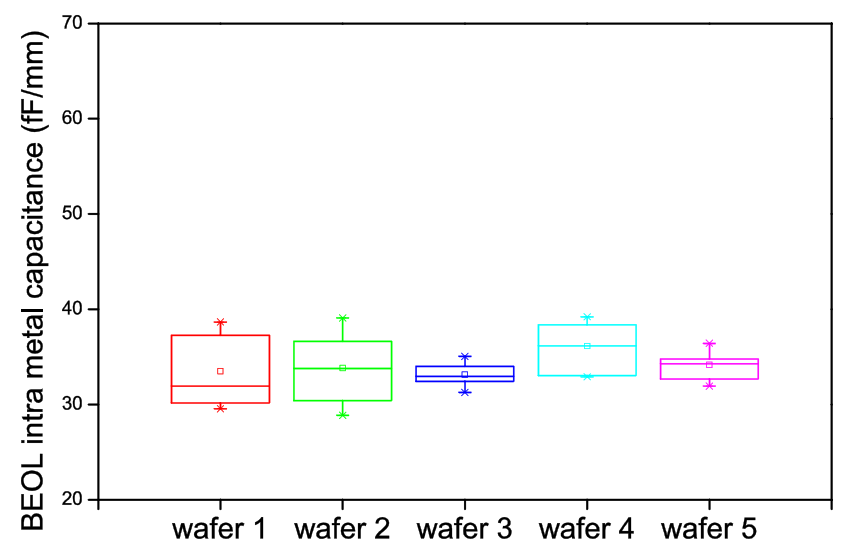

(b)

FIG. 44. Wafer-level (a) sheet resistance on M1/M2/M3/M4 and (b) intralayer capacitance on M1/M2/M3/M4. 


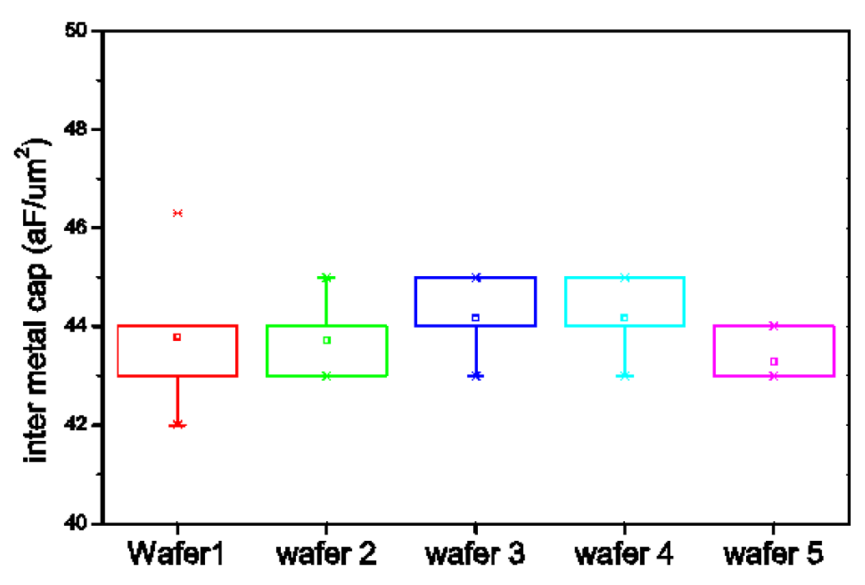

FIG. 45. Inter-metal capacitance between M1-M2, M2-M3, and M3-M4.

inter-layer capacitances. Figure 45 demonstrates wafer-level capacitance characterization between M1 and M2 layers, its average value is $43.8 \mathrm{aF} / \mathrm{um}^{2}$.

\section{Chip-to-chip link characterization}

Chip-to-chip connections on large size TSI $\left(40 \times 40 \mathrm{~mm}^{2}\right)$ can be as long as $10-25 \mathrm{~mm}$, especially to host GPU and multiple HBM. At transmitted data rates 2 Gbps, long interconnects behave as RF transmission lines. To characterize transmission line properties on the interposers, micro-strip lines and grounded-coplanar waveguides (G-CPW) in Figure 41(i) are implemented. Although multiple segments of "RC" models can be connected in series to model long interconnects, this model is only valid for narrow band estimation and transmission line theory is more preferred to fully characterize the behavior at high frequency. ${ }^{96}$ Interconnect physical cross-sections are designed for $50 \Omega$ impedance match. For example, $50 \Omega$ microstrip width of M4 layer is $10 \mu \mathrm{m}$ for the quasi-TEM mode propagation if M1 layer is assumed as an infinite ground plane. If a $50 \Omega$ microstrip lines is implemented in M2 or M3, the widths can be narrower compared to M4 microstrip line due to large parasitic capacitance. Furthermore, narrow width traces incur higher conductor loss, which degrades signal integrity. Their insertion lose $\left(\mathrm{S}_{21}\right)$ for interconnect lengths are simulated and experimentally verified via $S$-parameter measurement using the Vector Network Analyzer (VNA). The accepted frequency bandwidth for interconnects is generally limited by $3 \mathrm{~dB}$ insertion loss. ${ }^{97,98}$
In 2.5D TSIs, there are mainly two categories of connections: (a) on-TSI connections which link I/O pads of one chip on TSI to another chip's I/O pads on the same TSI and (b) off-TSI which link I/O pads of chips on TSI to the external world (organic substrate/PCB) via TSVs. For the on-TSI connections with length over $25 \mathrm{~mm}$, eye diagrams are measured with data rate of 2 Gbps. As seen in Figures 46(a) and 46(b), the signal integrity of RDL interconnect outperforms the BEOL interconnect due to lesser resistance and capacitance. The length and spacing $(\mathrm{L} / \mathrm{S})$ for BEOL interconnect here is $2 \mu \mathrm{m}$, its thickness is $1 \mu \mathrm{m}$, and $\mathrm{L} / \mathrm{S}$ for backside RDL interconnect is $3 \mu \mathrm{m}$, and its thickness is $3 \mu \mathrm{m}$.

TSV-back side RDL-TSV interconnect as shown in Figure 47(a) is analyzed for s-parameters using G-S-G structure illustrated in Figure 41(i). The S-parameter results characterized up to $40 \mathrm{GHz}$ are shown in Figure 47(b). Return loss is well below $12 \mathrm{~dB}$ for frequency below $40 \mathrm{GHz}$ indicating acceptable return loss. The single off-TSI insertion loss is half of overall TSV-backside RDL-TSV transition. Therefore, the single off-TSI interconnect insertion loss is $<2.5 \mathrm{~dB}$ up to $40 \mathrm{GHz}$. Besides the on-TSI interconnects, there are off-TSI interconnects through TSVs and backside micro-bumps. Figure 48 shows the measured eye diagram for $25 \mathrm{Gbps}$ data rate between front side of interposer and the backside interposer. The eye opening demonstrates that circuits, such as serializer/deserializer (SerDes), can be designed using the 2.5D TSI platform.

\section{Micro joint reliability characterization}

The reliability assessment of the micro-bump joints interconnecting the guest die and the TSI, as well as the backside micro-bump interconnecting the TSI on the substrate is critical to steer the technology mainstream. A daisy chain interlacing between the top metal of the TSI (M4) - to - front side micro-bump - to - top metal in the Guest die as shown in Figure 41(e) is an ideal structure of choice to perform the reliability assessment on micro-bump joints. Daisy chains with varying number of micro-bumps are constructed and evaluated for temperature-cycling ( $\sim 1000$ cycles $)$ as described in JEDEC standard. Similar daisy chains between the TSI and substrate shown in Figure 41(f) interlacing the back side $\mathrm{Cu}-\mathrm{RDL}$ - to - backside micro-bump - to - substrate trace are constructed and characterized to evaluate the reliability of the backside micro-bump. In technologies in which the backside RDL is not provided, the daisy chain

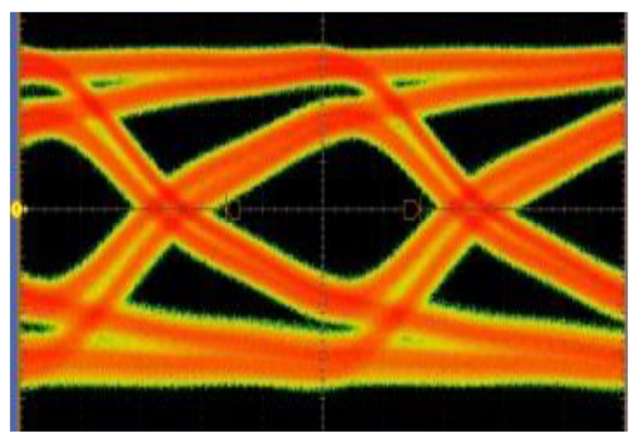

(a)

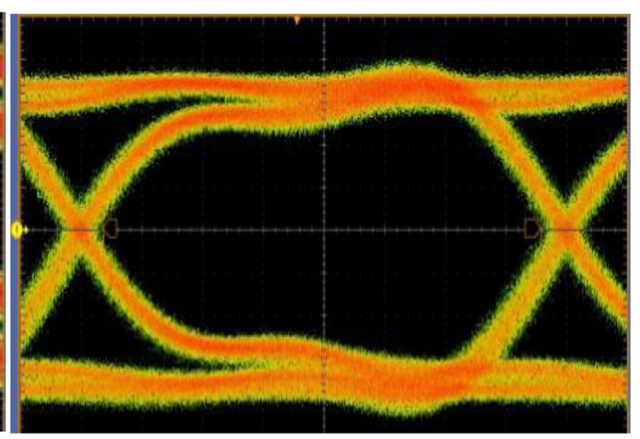

(b)
FIG. 46. Eye diagrams of 2 Gbps for $25 \mathrm{~mm}$ long (a) BEOL interconnect thickness $1 \mu \mathrm{m}$, width $2 \mu \mathrm{m}$, and pitch $4 \mu \mathrm{m}$ and (b) RDL interconnect thickness $3 \mu \mathrm{m}$, width $3 \mu \mathrm{m}$, and pitch $6 \mu \mathrm{m}$. 


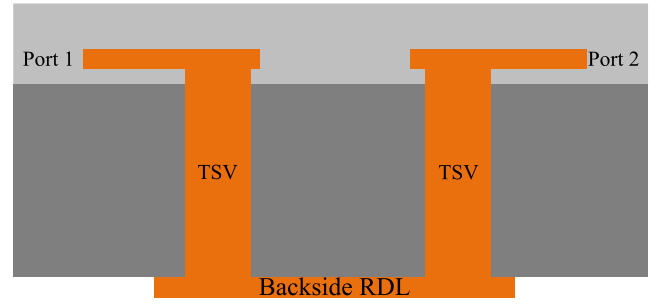

(a)

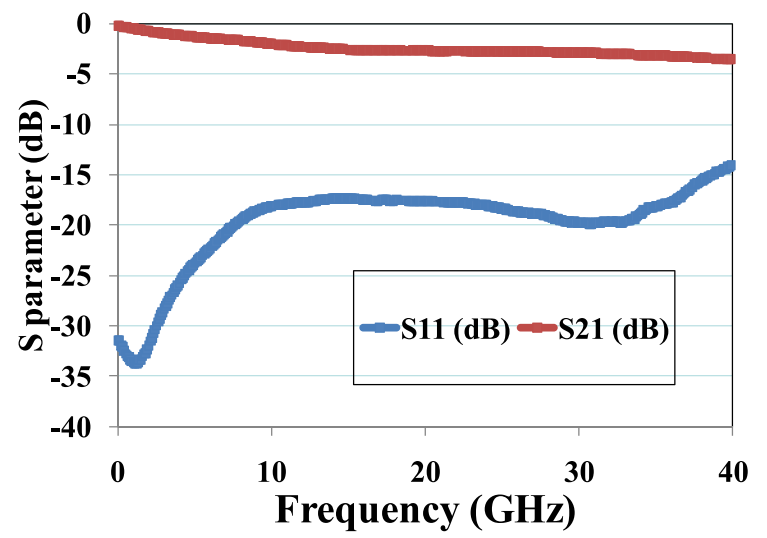

(b)

FIG. 47. (a) DUT and (b) S parameter characterization of TSV-back side RDL-TSV interconnect.

would interlace from M1 - to - TSV - to -backside microbump - to - PCB. It can be probed from PCB pads.

\section{Decoupling capacitors}

Clean power source is required for the ICs on $2.5 \mathrm{D}$ interposer. ${ }^{99}$ Voltage noise in power/ground planes disturbs the data in latch, logic error, data drop and false switching, and furthermore coupling to signals. Power fluctuation on power distribution network (PDN) must be kept in the allowable range. For example, the maximum allowable voltage deviation in FPGA core power and I/O buffers is typically $<5 \%$. Dedicated planes for power and ground are preferred compared to the power/ground grid. ${ }^{100}$ The decoupling capacitors temporarily store and provide the electrical charges when currents drawn by ICs fluctuate. Together with

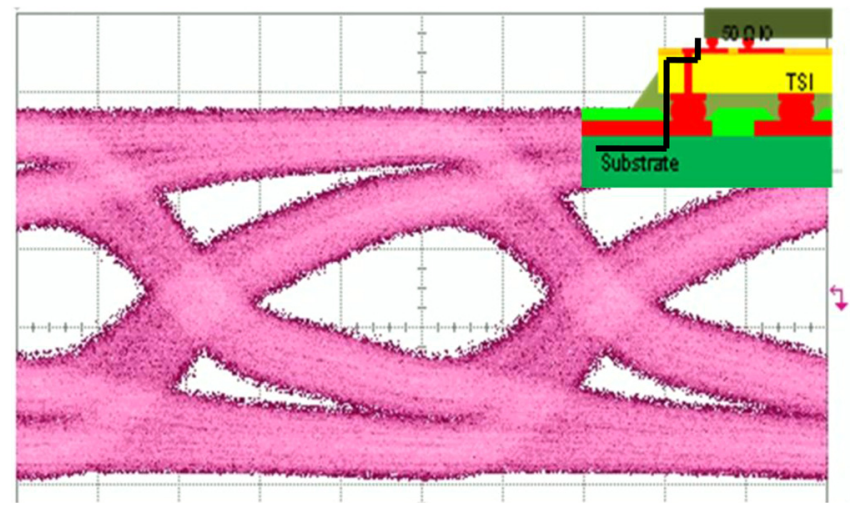

FIG. 48. Eye diagram of 25 Gbps for the off-TSI interconnect: M1-TSV-C4 bump. equivalent series inductance of PDN, decoupling capacitors function as a low-pass filter to suppress high frequency voltage fluctuation, and thus reduce the voltage noise contributed from the simultaneous transistor switching. As shown in Figure $49,{ }^{101}$ the package impedance $\mathrm{Z}$ around $100 \mathrm{MHz}$ is reduced due to $2.2 \mathrm{nF}$ capacitor integrated on interposer. Broad-level decoupling is often implemented with high quality ceramic capacitors range from $1 \mu \mathrm{F}$ to $10 \mu \mathrm{F}$. Those de-coupling capacitors need to be placed close to the targeted chips for optimal de-coupling effect. Die-level integrated high-density metal-insulator-metal (MIM) based on integrated passive device (IPD) technology on TSI are more effective than the package-level counterparts due to smaller equivalent series inductance (ESL). In Ref. 101, Altera demonstrated using on-TSI MIM capacitance in its $10 \mathrm{Gpbs}$ SerDes as shown in Figure 49. With the use of MIM capacitors the package impedance drops (indicating low impedance between power and ground). The improvements in the performance of transmitter jitter, eye height, and width were quite significant, as listed in Table V. The transmitter jitter, eye height, and width are improved by $10 \%, 20 \%$, and $7.7 \%$, respectively.

\section{THERMAL CONSIDERATIONS OF 2.5D PACKAGES}

\section{A. Thermal challenges in the context of 2.5D package}

Next generation of heterogeneous integration demands for $2.5 \mathrm{D}$ and $3 \mathrm{D}$ chips in one package for less signal latency, more functionality, and less power consumption. Nonetheless, there are tremendous obstacles in realizing the real 3D based vertical architecture with regards to fabrication and thermal management. Therefore, $2.5 \mathrm{D}$ package on TSI has been envisioned as the most viable strategy in the context of heterogeneous integration. In a typical 2.5D package, dissimilar chips such as logic, memory, and ASIC chips, as well as optical components such as laser diode and optical transceivers, can be assembled on an interposer. Wafer level packaging facilitates the manufacturability of the interconnection of multiple chips on the interposer.

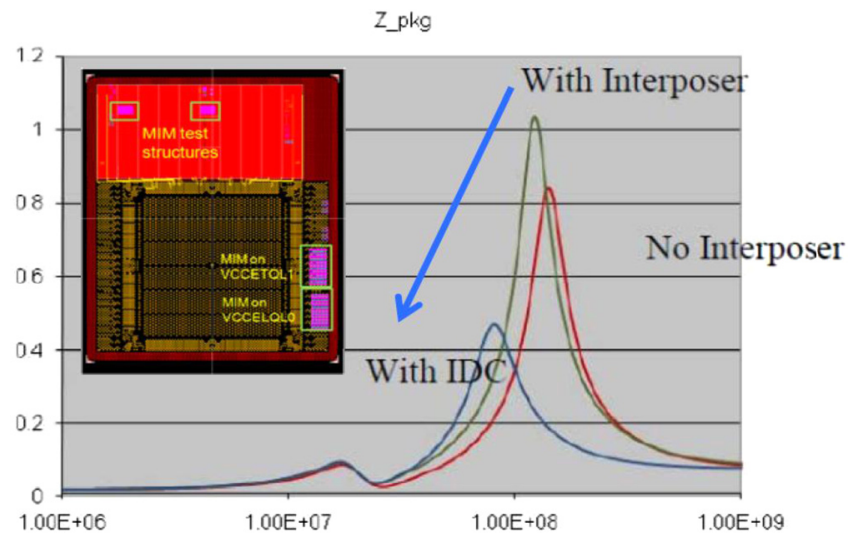

FIG. 49. Effect of decoupling capacitor for 2.5D IC power planes. ${ }^{101}$ Reproduced with permission from Zhe et al., "Development of an optimized power delivery system for 3D IC integration with TSV silicon interposer," in Proceedings of IEEE Electronic Components and Technology Conference (ECTC) (2012), pp. 678-682. Copyright 2012 Institute of Electrical and Electronics Engineers. 
TABLE V. Improvement in transmission Jitter, eye height and eye width due to the use of $2.2 \mathrm{nF}$ MIM CAP for 10 G SerDes. ${ }^{101}$ Reproduced with permission from Zhe et al., "Development of an optimized power delivery system for 3D IC integration with TSV silicon interposer," in Proceedings of IEEE Electronic Components and Technology Conference (ECTC) (2012), pp. 678-682. Copyright 2012 Institute of Electrical and Electronics Engineers.

\begin{tabular}{lcccccc}
\hline \hline & MIM (cap) & RJ (rms) $(\mathrm{ps})$ & DJ $(\mathrm{ps})$ & TJ (ps) & Eye height (mV) & Eye width (ps) \\
\hline 10 G SerDes & $2.2 \mathrm{nF}$ & 0.76 & 25.1 & 35.93 & 619 & 70 \\
& None & 0.77 & 28.16 & 39.2 & 512 & 65 \\
\hline \hline
\end{tabular}

The thermal management issues can be understood from two aspects. On one hand, the chip-level power delivery could reach significant levels up to $100 \mathrm{~W} / \mathrm{cm}^{2}$ for high processing chips, which require proximate cooling. Without compromising processing speed, the integration of multi-chips in one interposer on the package tends to generate higher heat density, which needs to be addressed in the $2.5 \mathrm{D}$ or $3 \mathrm{D}$ package design. ${ }^{102-115}$ A schematic of the cooling concepts is illustrated in Figure 50.

On the other hand, the temperature sensitive chips, such as optical chips, are more prone to wavelength shifts due to the temperature excursions, therefore requiring temperature control. ${ }^{116}$ While the additional interposer may help to even out the hot spots, it may also introduce thermal cross-talk between chips requiring different operation temperature. To mitigate against thermal cross-talk, thermal management techniques, such as guide ring/heaters or thermoelectric cooling, can be implemented to avoid transmission bit error. ${ }^{117-120}$

In the past decade, substantial efforts have been made to address the thermal management issues and challenges, including passive cooling characterization, thermal enhancement due to Si carriers, thermal enhancement with liquid cooled cold plate, or even microchannel heat sinks built on the backside of chips. The cooling of 3D stacked packages with on chips on Si carriers is studied in Ref. 105. It was identified that, due to package limitation, only $1 \mathrm{~W}$ could be dissipated from the two chips stacked on their chip carriers through microbump joining. Due to insufficient passive cooling capacity downward through the PCB, additional cold plate was used to spread the heat from the top of the stack. It is identified that $20 \mathrm{~W}$ can be dissipated through the chip carrier, with maximum temperature rise of $48.9^{\circ} \mathrm{C}$. A direct liquid cooling of three stacked chips on $\mathrm{Si}$ carriers is presented in Ref. 106, which shows that direct liquid cooling

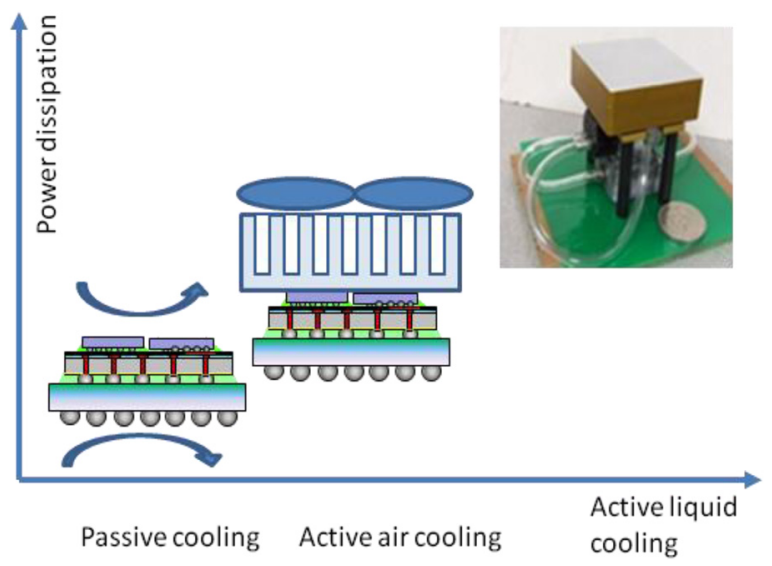

FIG. 50. Schematic of the cooling concepts. can dissipate $23 \mathrm{~W}$ from the chip carriers based on dielectric fluid. Recent research is conducted on the thermal management of two stacked chips on Si carriers cooled by microchannel heat sinks fabricated in the chip carriers. ${ }^{107,108} \mathrm{~A}$ heat flux of $100 \mathrm{~W} / \mathrm{cm}^{2}$ can be dissipated from each chip stack through dual in-port fluidic design.

With more attention paid to the $2.5 \mathrm{D}$ package with heterogeneous integration, research work has been conducted on the effect of Si interposer on the thermal performance of the package. ${ }^{99-102}$ A comprehensive thermal characterization is conducted on the TSI package with bare-die and overmolded packages. ${ }^{112}$ The measured thermal resistance (Theta JA) is $35 \mathrm{~K} / \mathrm{W}$ under natural convection. When the liquid-cooled cold plate is applied, the thermal resistance drops to $0.82 \mathrm{~K} / \mathrm{W}$ from the same chip, validating the cold plate's efficiency in removing heat from the $2.5 \mathrm{D}$ package. The respective cooling performance will be discussed in Secs. V B-V D.

\section{B. Passive cooling}

Passive cooling is defined here as the cooling techniques that do not use any active fluid movers such as pumps and cooling fans. Conventionally, thermally enhanced PCB, lid encapsulation are used as passive cooling techniques. 2.5D package normally consists of several chips on $\mathrm{Si}$ or glass interposer with TSVs for electrical interconnections. Compared with conventional package, the 2.5D package has different thermal behavior since interposer with through vias serves as heat spreader for heat dissipation in the absence of active cooling apparatus such as axial fans. Thermal characterization can be conducted under still air condition to extract thermal metrics such as Theta JA or Theta JB. The passive cooling is especially useful in space-tight and energy-limiting applications, e.g., tablets and smart phones. The thermal performances with different design factors for the interposer and TSVs are outlined in Secs. V B 1-V B 4.

\section{TSV thermal enhancement}

A forest of TSV will have an impact on the interposer thermal performance. The approach for extracting the equivalent thermal conductivity is shown in Figure 51 for modeling of equivalent thermal conductivities of TSV interposer along the through plane $\mathrm{z}$ direction and in-plane $\mathrm{x}$ and $\mathrm{y}$ directions. ${ }^{109}$ First, create the geometry of TSV interposer with different TSV design parameters, then set the boundary conditions and simulate the temperature distribution. After obtaining the temperature distribution, the equivalent thermal conductivity can be calculated. The average temperature 


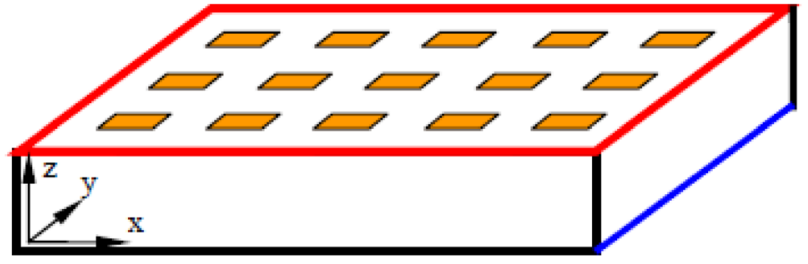

(a) Modeling equivalent thermal conductivity of TSV interposer

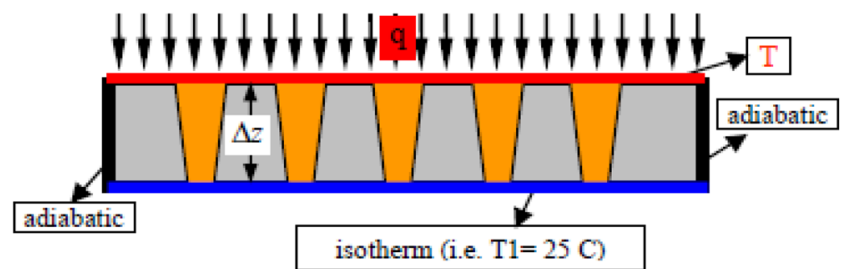

(b) Method for extracting equivalent thermal conductivity in $z$ direction

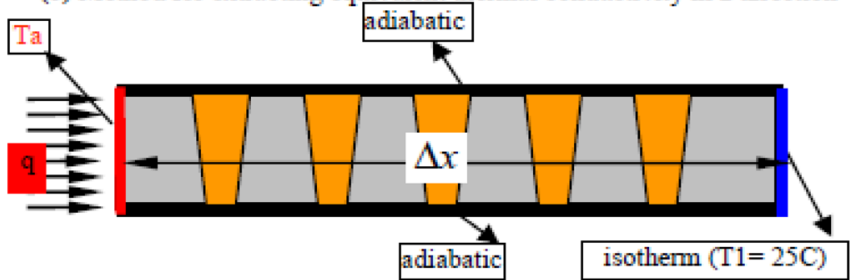

(c) Method for extracting equivalent thermal conductivity in $\mathrm{x}$ and $\mathrm{y}$ directions

FIG. 51. Equivalent thermal conductivity extraction models for TSV interposer: (a) Modeling equivalent thermal conductivity of TSV interposer, (b) method for extracting equivalent thermal conductivity in $\mathrm{z}$ direction, and (c) method for extracting equivalent thermal conductivity in $\mathrm{x}$ and $\mathrm{y}$ directions. ${ }^{109}$ Reproduced with permission from Hoe et al., "Effect of TSV interposer on the thermal performance of FCBGA package," in 11th IEEE Electronics Packaging Technology Conference (EPTC) (2009). Copyright 2009 Institute of Electrical and Electronics Engineers.

on the top surface is obtained, and consequently, the equivalent thermal conductivity can be calculated as follows:

$$
\begin{gathered}
q=-k_{e q, z} \frac{\Delta T}{\Delta z}, \\
k_{e q, z}=-q\left(\frac{\Delta z}{\Delta T}\right), \\
q=-k_{e q, x} \frac{\Delta T}{\Delta x}, \\
k_{e q, x}=-q\left(\frac{\Delta x}{\Delta T}\right) .
\end{gathered}
$$

The geometrical dimensions and thermal properties used for detailed modeling are listed in Table VI.
TABLE VI. TSV interposer parameters and thermal properties.

\begin{tabular}{lcc}
\hline \hline Component & Interposer & TSV \\
\hline Material & Silicon & Copper \\
Thermal conductivity (W/mK) & 150 & 390 \\
TSV pitch (mm) & \multicolumn{2}{c}{$0.15-0.6$} \\
TSV via ratio & $2-10$ & \\
TSV cu plating thickness (urn) & $5-25$ \\
TSV filler material & No filler, polymer based. Aluminum, Copper
\end{tabular}

a. Equivalent thermal conductivity with $\mathrm{Cu}$ filled TSVs. Figure 52 shows five different TSVs with various aspect ratios $\mathrm{A}$, which $\mathrm{A}$ is defined as $\mathrm{A}=$ Thickness/Average diameter of TSV. The thickness of the wafer is $300 \mu \mathrm{m}$, and the etching angle of the tapered Cu-filled TSV is $85^{\circ}$. Figures 53-55 show that the equivalent thermal conductivity of the TSV interposer varies with TSV aspect ratios and pitches for equivalent thermal conductivity in the normal $\mathrm{z}$-direction, keq, $\mathrm{z}$, and equivalent thermal conductivity in the planar directions ( $\mathrm{x}$ and $\mathrm{y}$ directions), keq, $\mathrm{x}=\mathrm{keq}, \mathrm{y}$, respectively. It can be seen that for a fixed pitch, the equivalent thermal conductivity of Cu-filled TSV interposer increases when the aspect ratio decreases, which is especially sensitive when the aspect ratio is small-ranging from 2 to 4, and the equivalent thermal conductivity in all directions is greater for larger diameters of the TSV. For engineering convenience, the results have been curve-fitted into the following empirical equations for the equivalent thermal conductivity:

$$
\begin{gathered}
k_{e q, z}=150+188 D^{2} P^{-2}, \\
k_{e q, x}=k_{e q, y}=150+105 D^{2} P^{-2} .
\end{gathered}
$$

b. Equivalent thermal conductivity with TSVs filled with varying materials. Figure 56 shows the TSV plated with thin layer of copper. Figures 57 and 58 show the variation of equivalent thermal conductivity of TSV interposer with the partially plated copper thickness for different TSV pitch, respectively, in the normal direction (z-direction) and in the planar directions ( $x$ and $y$-direction). It can be seen that equivalent thermal conductivities of the TSV interposer increase with the plating thickness. In addition, the equivalent thermal conductivities of the TSV interposer are more sensitive to the copper plating thickness for smaller pitches of vias. For example, in the case of $0.15 \mathrm{~mm}$ TSV pitch, the equivalent thermal conductivity in the $\mathrm{z}$-direction increases

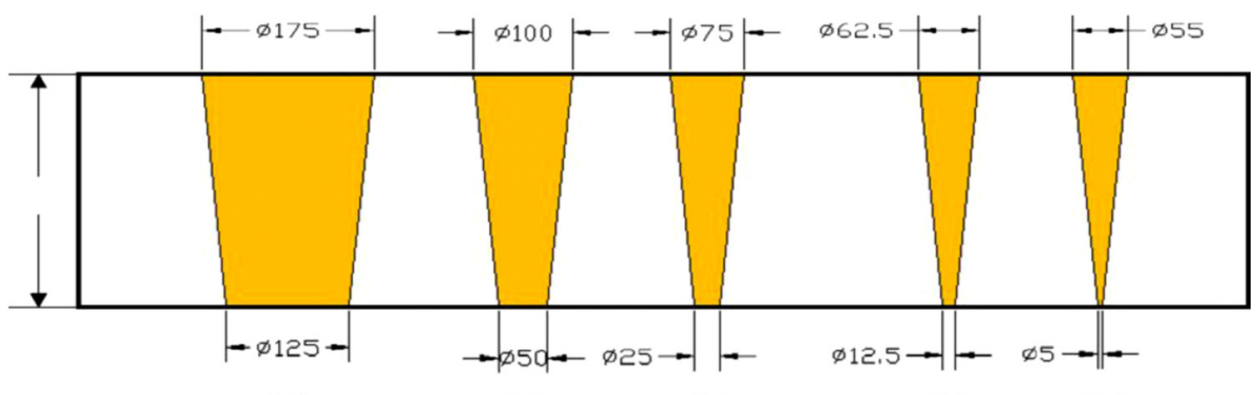

$2: 1$
$4: 1$

$6: 1$
$8: 1$

$10: 1$
FIG. 52. TSV geometry for different aspect ratios. ${ }^{109}$ Reproduced with permission from Hoe et al., "Effect of TSV interposer on the thermal performance of FCBGA package," in 11th IEEE Electronics Packaging Technology Conference (EPTC) (2009). Copyright 2009 Institute of Electrical and Electronics Engineers. 


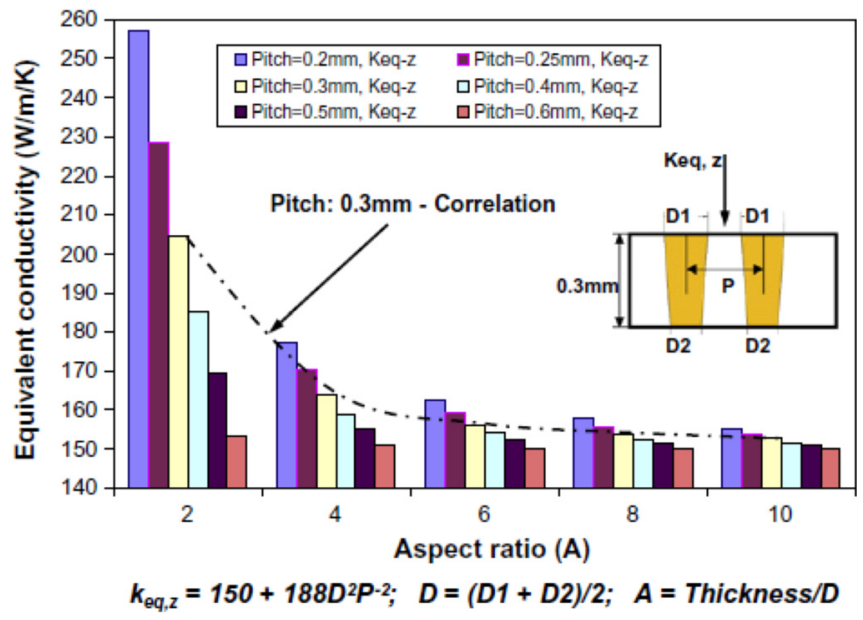

FIG. 53. Equivalent thermal conductivity in normal z-direction (keq,z). ${ }^{109}$ Reproduced with permission from Hoe et al., "Effect of TSV interposer on the thermal performance of FCBGA package," in 11th IEEE Electronics Packaging Technology Conference (EPTC) (2009). Copyright 2009 Institute of Electrical and Electronics Engineers.

from $138 \mathrm{~W} / \mathrm{mK}$ to $187 \mathrm{~W} / \mathrm{mK}(\sim 40 \%)$ if the $\mathrm{Cu}$ plating thickness is increased from $5 \mu \mathrm{m}$ to $25 \mu \mathrm{m}$. For the simulated interposer with a fixed size of $25 \mathrm{~mm} \times 25 \mathrm{~mm}$, the finer the pitch, and hence, more TSVs incorporated in the interposer, results in more air or copper material and less silicon material in the interposer and thus a greater influence of the air or copper on the equivalent thermal conductivity. As to the case of $5 \mu \mathrm{m} \mathrm{Cu}$ thickness, smaller via pitch with smaller copper thickness results in more TSVs with more air, but less copper and silicon material. The incorporated more air vias account for the lowest thermal conductivity. By increasing the thickness of copper, more copper can be incorporated due to more TSVs included in the interposer, which suggesting better thermal conduction can be enabled. This is also the reason why TSV interposer with smaller pitch is more sensitive to the copper plating thickness.

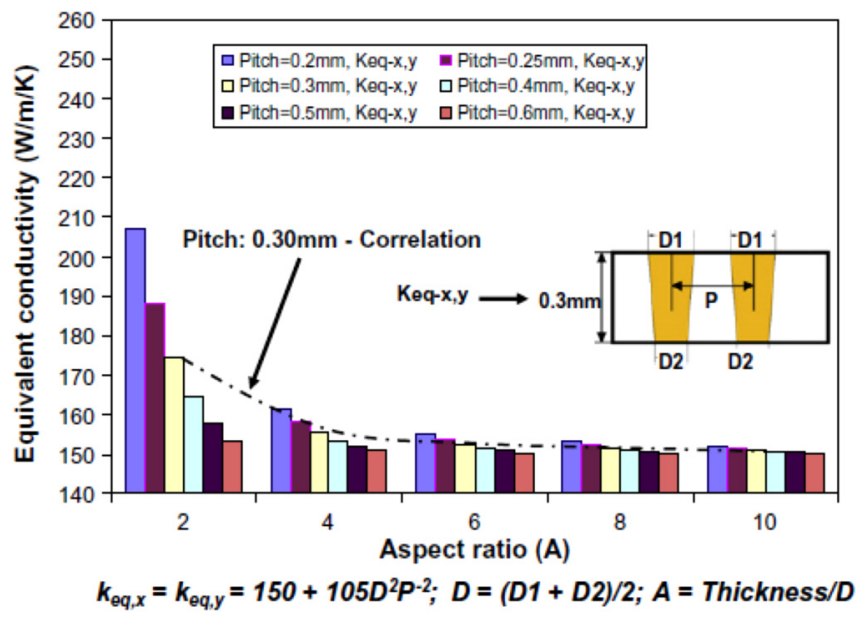

FIG. 54. Equivalent thermal conductivity in normal $\mathrm{x}, \mathrm{y}$-direction (keq,x) and (keq,y) ${ }^{109}$ Reproduced with permission from Hoe et al., "Effect of TSV interposer on the thermal performance of FCBGA package," in 11th IEEE Electronics Packaging Technology Conference (EPTC) (2009). Copyright 2009 Institute of Electrical and Electronics Engineers.

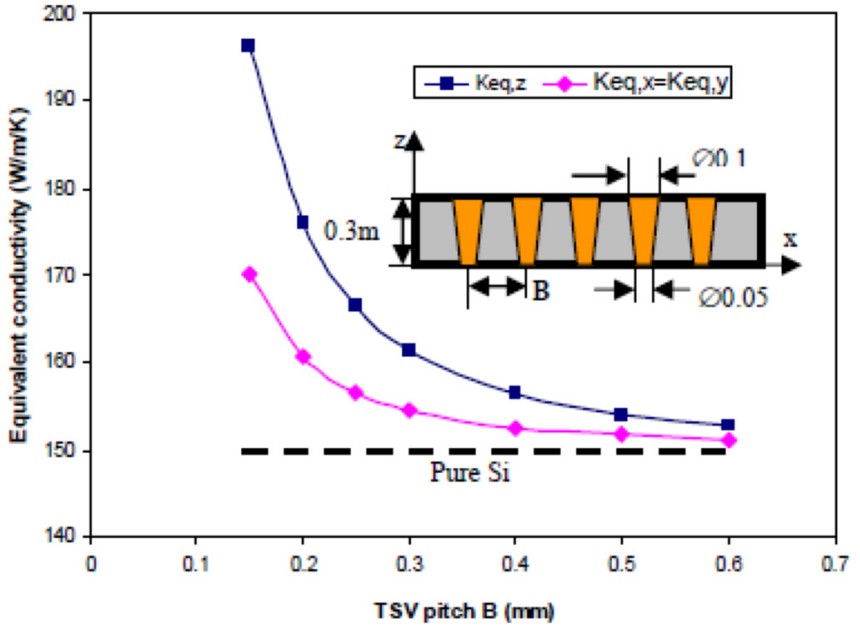

FIG. 55. Equivalent thermal conductivity of TSV interposer with different TSV pitches. ${ }^{109}$ Reproduced with permission from Hoe et al., "Effect of TSV interposer on the thermal performance of FCBGA package," in 11th IEEE Electronics Packaging Technology Conference (EPTC) (2009). Copyright 2009 Institute of Electrical and Electronics Engineers.

\section{Effect of TSV interposer on package thermal performance}

Figure 59 shows the compact modeling of the 3D SiP with the TSVs in interposer using a compact block with the pre-determined equivalent thermal conductivities. This model consists of a heat generating chip, a TSV interposer, a BGA build-up substrate, a PCB, and the solder joints. Their dimensions and thermal properties are listed in Table VII.

The junction to ambient thermal resistance of the package $\left(R_{j a}\right)$ can be expressed as $R_{j a}=\left(T_{j}-T_{a}\right) / P$, where $T_{j}$ and $T_{a}$ represent the junction temperature and ambient temperature, respectively, while $\mathrm{P}$ refers to the total power dissipated from the chip.

Figure 60 shows the junction to ambient thermal resistance of packages with different chip power dissipations. It can be seen that: (1) the thermal resistance reduces as the chip power increases (this is because high chip power induces high package temperature, as such high heat transfer coefficient is induced and more heat is removed from the package), and (2) the thermal resistance of the TSV package is lower than that of the package without TSV (this is because of the TSV interposer's spreading effect). The dashed lines in Figure 60 represent the correlations between the thermal resistance and chip power when the junction temperature equals to $85^{\circ} \mathrm{C}$ and $125^{\circ} \mathrm{C}$, respectively. ${ }^{109}$

Figure 61 shows the effect of the TSV interposer area on the resistance $\left(\mathrm{R}_{\mathrm{ja}}\right)$ decreases about $14 \%$ when the TSV interposer size increases from $21 \mathrm{~mm} \times 21 \mathrm{~mm}$ to $45 \mathrm{~mm} \times 45 \mathrm{~mm}$. This indicates that package thermal performance can be improved by increasing the TSV interposer size.

Figure 62 shows the effect of the TSV interposer thickness on the package thermal performance. It can be seen that the thicker the TSV interposer the lower the thermal resistance. This is because of the increasing spreading effect with thicker interposer/ chip. However, for small size interposers, this effect is negligible. 


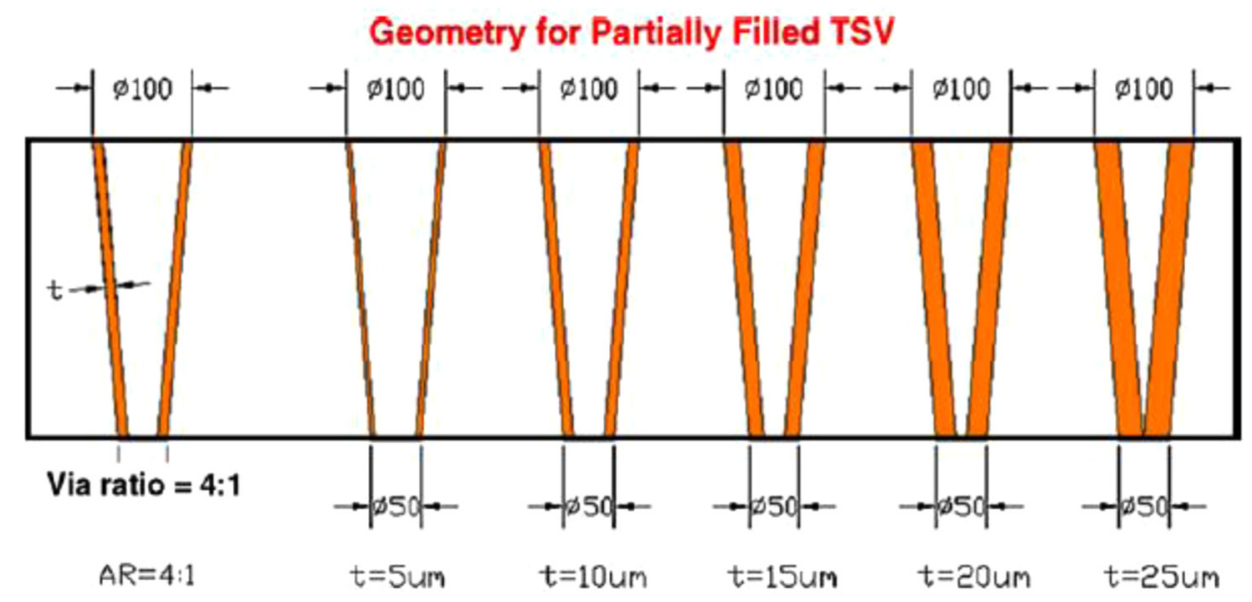

FIG. 56. Geometry for partially filled TSV. ${ }^{109}$ Reproduced with permission from Hoe et al., "Effect of TSV interposer on the thermal performance of FCBGA package," in 11th IEEE Electronics Packaging Technology Conference (EPTC) (2009). Copyright 2009 Institute of Electrical and Electronics Engineers.

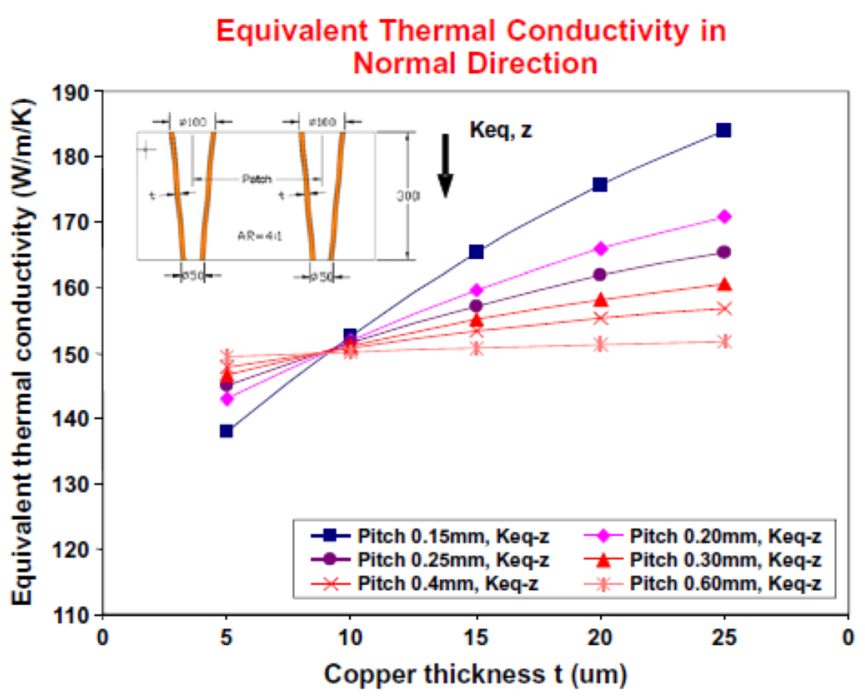

FIG. 57. Equivalent thermal conductivity Keq,z for partially filled TSV. ${ }^{109}$ Reproduced with permission from Hoe et al., "Effect of TSV interposer on the thermal performance of FCBGA package," in 11th IEEE Electronics Packaging Technology Conference (EPTC) (2009). Copyright 2009 Institute of Electrical and Electronics Engineers.

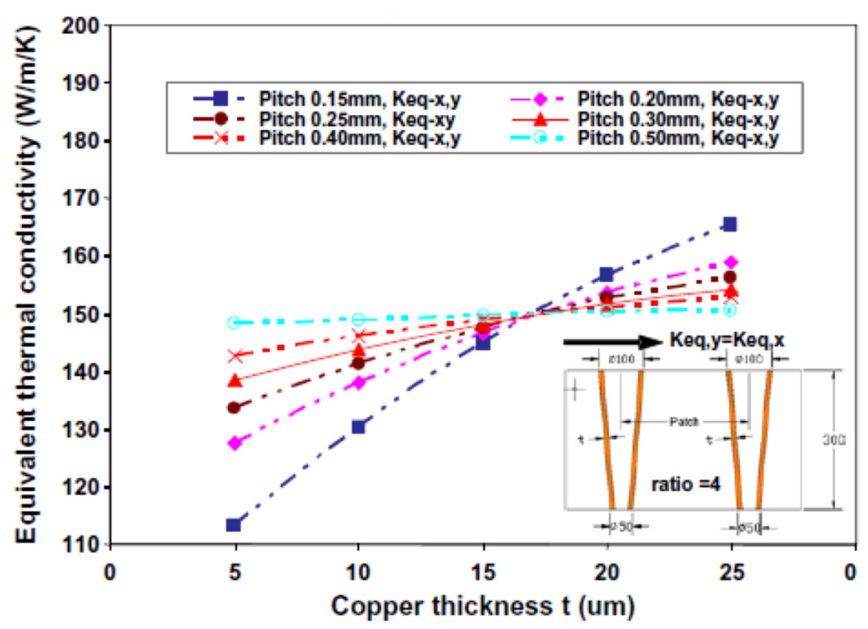

FIG. 58. Equivalent thermal conductivity Keq,x (=Keq,y) for partially filled TSV. ${ }^{109}$ Reproduced with permission from Hoe et al., "Effect of TSV interposer on the thermal performance of FCBGA package," in 11th IEEE Electronics Packaging Technology Conference (EPTC) (2009). Copyright 2009 Institute of Electrical and Electronics Engineers.
Figure 63 shows the effect of the chip size for different size ratios of the TSV interposer to the chip on the thermal performance of the package. It can be seen that for the same chip to TSV interposer size ratio (b/a), the smaller sizes of chips induce higher thermal resistance of the package.

\section{Thermal characterization of stacked chips}

The thermal characterization for $2.5 \mathrm{D} / 3 \mathrm{D}$ packages with TSVs can be conducted under natural convection condition. A schematic of the 3D stack package developed is shown in Figure 64. The 3D package overall dimensions are $13.5 \mathrm{~mm} \times 13.5 \mathrm{~mm} \times 1.4 \mathrm{~mm}$, having 276 peripheral I/Os. Carrier 1 is designed to mount a flip-chip (chip 1) representing an ASIC device. The chip 1 dimensions are $8.5 \mathrm{~mm}$ $\times 8.5 \mathrm{~mm} \times 0.1 \mathrm{~mm}$ with $1000 \mathrm{I} / \mathrm{Os}$ at a bump pitch of $0.25 \mathrm{~mm}$. Carrier 2 is designed to mount two memory chips, chip 2 and chip 3, each having overall dimensions of $4.5 \mathrm{~mm} \times 9.0 \mathrm{~mm} \times 0.1 \mathrm{~mm}$ and $80 \mathrm{I} / \mathrm{Os}$. The two Si carriers were stacked one over other using $8 \mathrm{mil}$ solder balls. Electrical connections through the Si carrier were formed by TSV technology. Some of the advantages of using Si for the interposer are low thermo-mechanical stress, fine line width and spacing, wafer level batch fabrication, and high thermal conductivity.

Thermal characterization requires a test die with heater and temperature sensor. It was difficult to procure test die similar to the die dimensions used in this study. We used a

Schematic Diagram and Modeling of the

SiP

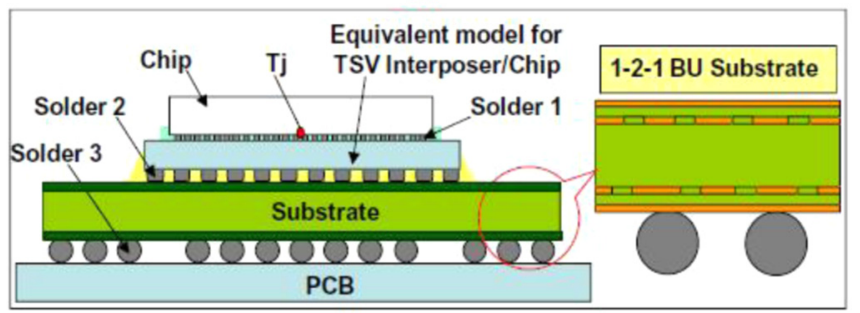

FIG. 59. Schematic diagram and modeling of SiP. ${ }^{109}$ Reproduced with permission from Hoe et al., "Effect of TSV interposer on the thermal performance of FCBGA package," in 11th IEEE Electronics Packaging Technology Conference (EPTC) (2009). Copyright 2009 Institute of Electrical and Electronics Engineers. 
TABLE VII. Geometry details and material properties for the package. ${ }^{109}$ Reproduced with permission from Hoe et al., "Effect of TSV interposer on the thermal performance of FCBGA package," in 11th IEEE Electronics Packaging Technology Conference (EPTC) (2009). Copyright 2009 Institute of Electrical and Electronics Engineers.

\begin{tabular}{lccc}
\hline \hline Component & Material & $\begin{array}{c}\text { Thermal conductivity } \\
(\mathrm{W} / \mathrm{m} / \mathrm{C})\end{array}$ & $\begin{array}{c}\text { Dimension } \\
(\mathrm{mm})\end{array}$ \\
\hline Chip & $\mathrm{Si}$ & 150 & $21 \times 21 \times 0.75$ \\
Solder 1 & $\mathrm{SnAg}$ & 57 & Pitch: 0.15 \\
& & & Height: 0.08 \\
& & & Aver D: 0.08 \\
Variable \\
Interposer & $\mathrm{S}_{4}+\mathrm{TSV}(\mathrm{Cu})$ & $\mathrm{Keq}$ & Pitch: 0.5 \\
Solder 2 & SnAg & 57 & Height: 0.1 \\
& & & Aver D: 0.1 \\
Substrate & Ituikhip & $/ / 100 \perp 0.5$ & $1-2-1(45 \times 45 \times 1)$ \\
Solder 2 & SaAg & 57 & Pitch: 1.0 \\
& & & Height: 0.6 \\
& & & Aver D: 0.6 \\
PCB & FR4 & $/ / 0.8 \perp 0.3$ & $101 \times 114 \times 1.6$ \\
\hline \hline
\end{tabular}

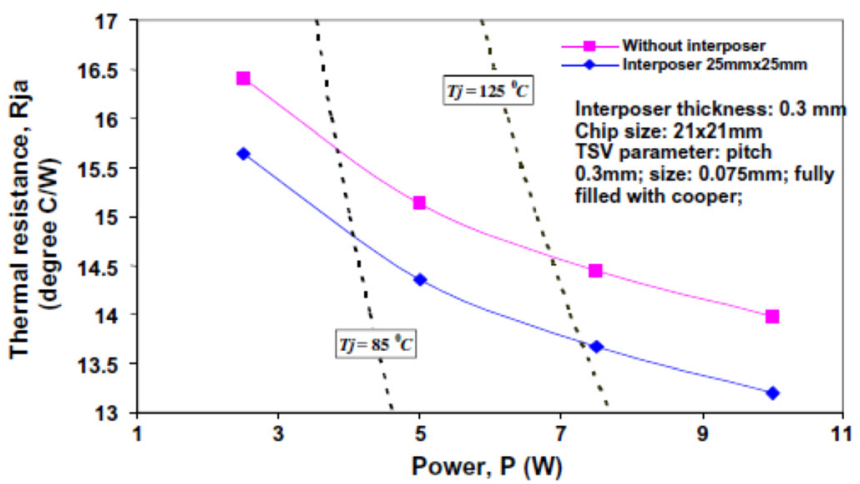

FIG. 60. Junction to ambient thermal resistance for different power dissipations. ${ }^{109}$ Reproduced with permission from Hoe et al., "Effect of TSV interposer on the thermal performance of FCBGA package," in 11th IEEE Electronics Packaging Technology Conference (EPTC) (2009). Copyright 2009 Institute of Electrical and Electronics Engineers.

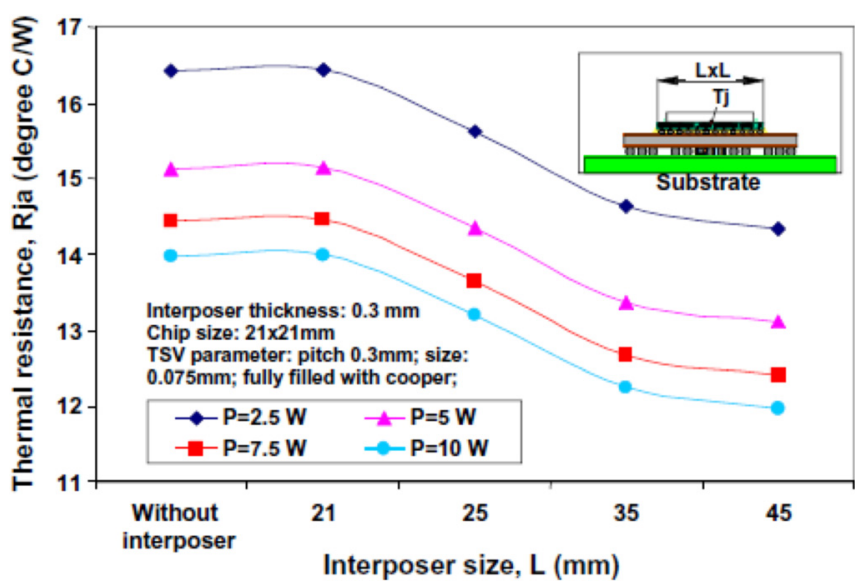

FIG. 61. Junction to ambient thermal resistance for different TSV interposer size. ${ }^{109}$ Reproduced with permission from Hoe et al., "Effect of TSV interposer on the thermal performance of FCBGA package," in 11th IEEE Electronics Packaging Technology Conference (EPTC) (2009). Copyright 2009 Institute of Electrical and Electronics Engineers.

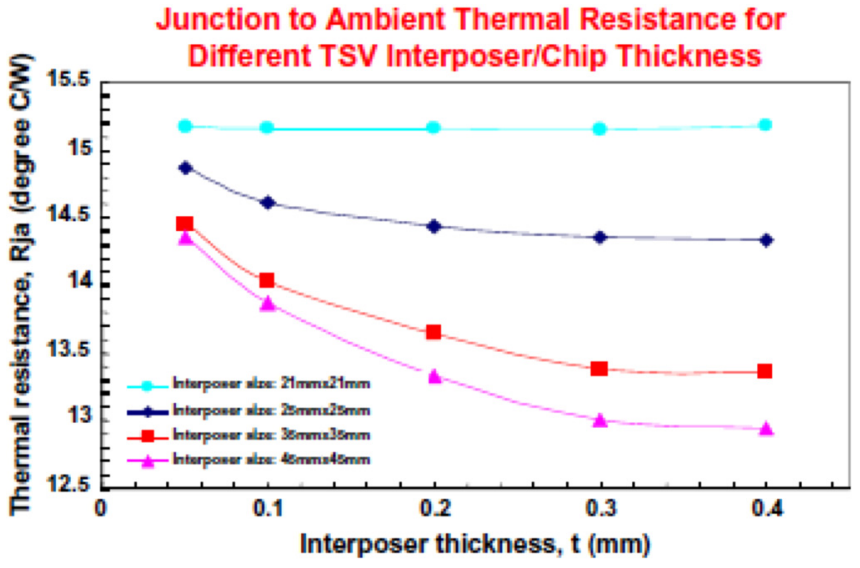

FIG. 62. Junction to ambient thermal resistance for different TSV interposer thickness. ${ }^{109}$ Reproduced with permission from Hoe et al., "Effect of TSV interposer on the thermal performance of FCBGA package," in 11th IEEE Electronics Packaging Technology Conference (EPTC) (2009). Copyright 2009 Institute of Electrical and Electronics Engineers.

Junction to Ambient Thermal Resistance for Different TSV Parameters

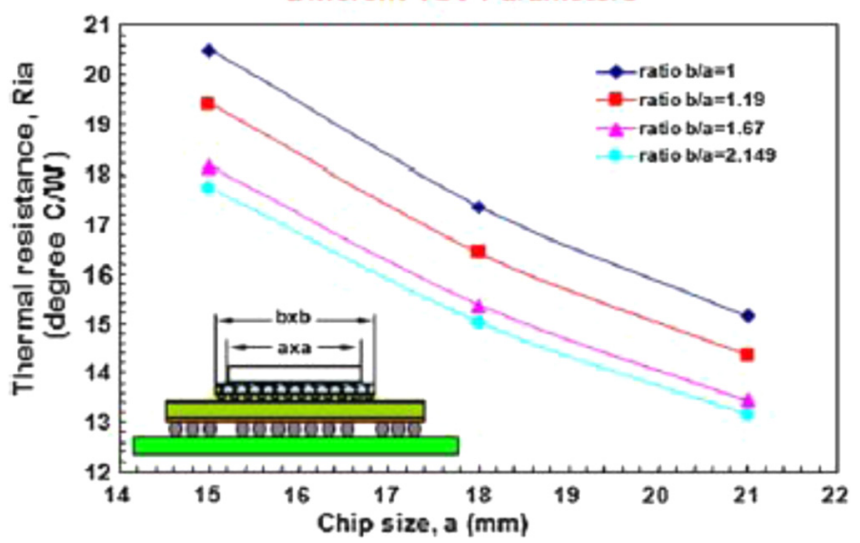

FIG. 63. Junction to ambient thermal resistance for different TSV sizes at different chip sizes. ${ }^{109}$ Reproduced with permission from Hoe et al., "Effect of TSV interposer on the thermal performance of FCBGA package," in 11th IEEE Electronics Packaging Technology Conference (EPTC) (2009). Copyright 2009 Institute of Electrical and Electronics Engineers.

(a)

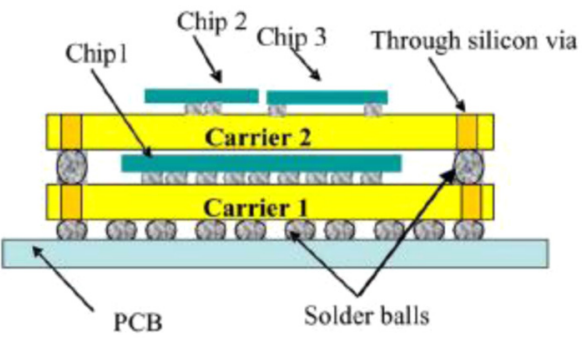

(b)

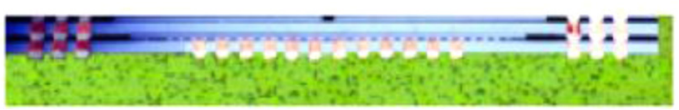

FIG. 64. (a) Schematic of 3D stack package and (b) cross section of 3D stack package. 


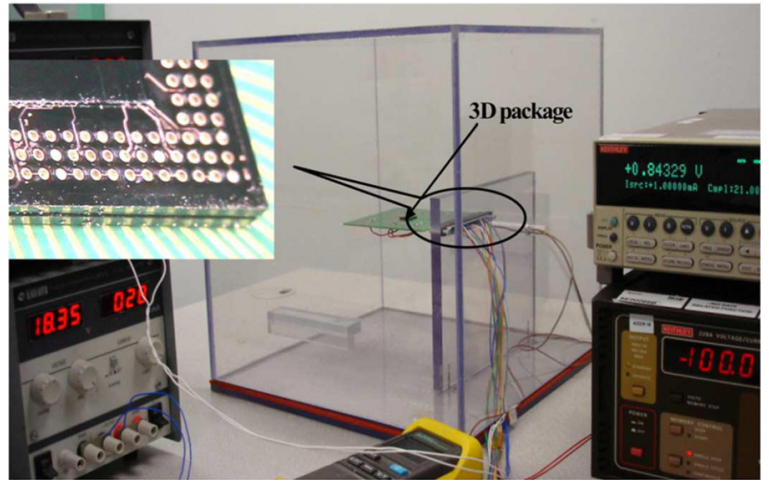

FIG. 65. Experimental characterization of thermal resistance under natural convection condition.

test die available in our laboratory, which has dimensions of $8.9 \mathrm{~mm}$. The test die was thinned to $100 \mu \mathrm{m}$. Each carrier was assembled with test die. The two carriers were stacked one over another using $\mathrm{SnPb}$ eutectic solder balls. The 3D package was assembled onto a four-layer test board. Figure 65 shows the assembled board mounted horizontally inside an enclosed chamber for natural convection thermal characterization as defined in JESD-51 standards. ${ }^{121,122}$ The test chips in carrier 1 and carrier 2 were connected to a separate power source and supplied with $0.8 \mathrm{~W}$ and $0.2 \mathrm{~W}$, respectively. Four such packages were characterized, and the average of maximum temperature chip 1 is compared. The maximum temperature increase of chip 1 was $24.2^{\circ} \mathrm{C}$, with calculated thermal resistance of $30.3 \mathrm{~K} / \mathrm{W}$ based on $0.8 \mathrm{~W}$ power input.

Two types of thermal enhancements techniques were studied, i.e., through thermal via and thermal bridging using polymer adhesive. Thermal analysis was carried out with a heat load of $2.8 \mathrm{~W}$ in chip 1 and $0.1 \mathrm{~W}$ each in chip 2 and chip 3, respectively. First, the package thermal performance was studied with 144 thermal vias in the carrier 1 . Maximum temperature of chip 1 with thermal via is $77.5^{\circ} \mathrm{C}$ as compared to $78.5^{\circ} \mathrm{C}$ without thermal vias. $\mathrm{Si}$ has good thermal conductivity, therefore, thermal via in the Si carrier is not effective in reducing the package thermal resistance. Another thermal enhancement was done by bridging the gap between chip 1 and carrier 2 using thermal adhesive. A polymer adhesive having thermal conductivity of $2 \mathrm{~W} / \mathrm{mK}$ was used to bridge the chip 1 and carrier 2 . The maximum temperature of chip 1 with thermal adhesive is found to be $73.9^{\circ} \mathrm{C}$. The thermal resistance is calculated to be $17.5 \mathrm{~K} / \mathrm{W}$. It is seen that the thermal bridging helps to reduce the thermal resistance by $42 \%$ for the chip 1 through the upward thermal path on the carrier 2 by forming a parallel heat flow path. Further improvement may include the heat spreader and cold plate at the top, which however can be categorized into the active cooling technology. It has been shown by thermal simulation that $20 \mathrm{~W}$ could be dissipated from chip 1 through the top cold plate cooling method.

\section{Thermal characterization of 2.5D package}

A thorough thermal characterization of the $2.5 \mathrm{D}$ package is done in Ref. 112. The thermal resistance of Theta JA,

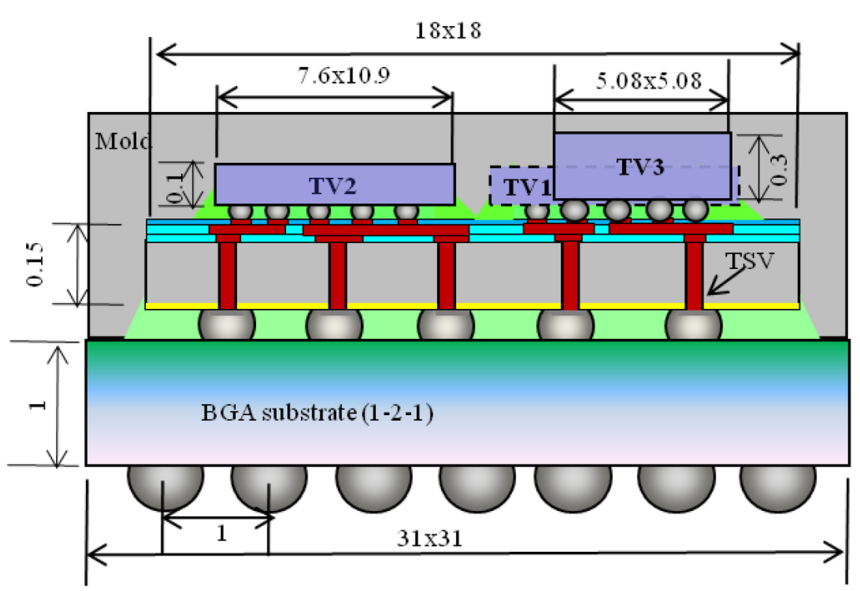

FIG. 66. The schematic of the multi chip module on through Si interposer, with TV1 behind TV3. The SEM image of TSV under thermal chip bumps is also shown. (Unit: mm). ${ }^{112}$ Reproduced with permission from Zhang et al., "Thermal characterization and simulation study of $2.5 \mathrm{D}$ packages with multi-chip module on through silicon interposer," in 15th IEEE Electronics Packaging Technology Conference (EPTC) (2013). Copyright 2013 Institute of Electrical and Electronics Engineers.

Theta JB, and Theta JC are characterized and compared with the thermal simulation. The concept of the package structure is shown in Figure 66 and the actual packages, both molded and bare die types, are shown in Figure 67 . The thermal test die with a size of $5.08 \mathrm{~mm} \times 5.08 \mathrm{~mm}$ is laid out on the $\mathrm{Si}$ interposer, together with two dummy dies with sizes of $7.6 \mathrm{~mm} \times 10.9 \mathrm{~mm}, 8 \mathrm{~mm} \times 8 \mathrm{~mm}$. All the dies are fabricated and assembled on the same interposer wafer through microbumps and underfill processes. The two dummy dies represent the logic and memory chips in a typical TSI integration. The interposer wafer was fabricated through processes including TSV, copper filling, and top-side RDL processes on 12 in. wafer to form micro straight vias of $10 \mu \mathrm{m}$ in diameter and $100 \mu \mathrm{m}$ deep through the interposer. The interposer of $18 \mathrm{~mm} \times 18 \mathrm{~mm} \times 0.1 \mathrm{~mm}$ in size was then assembled

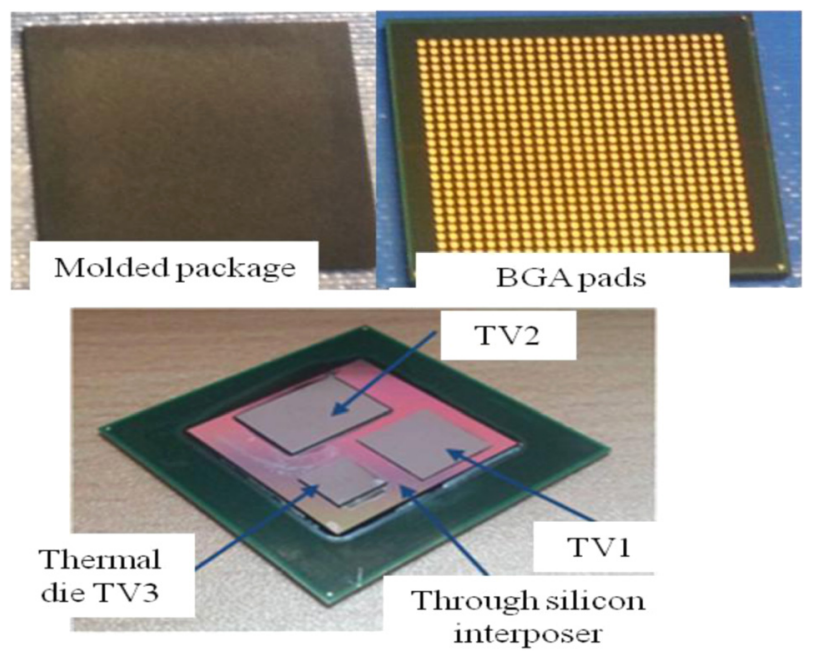

FIG. 67. The molded and bare die TSI package in $26 \times 26$ BGA format. ${ }^{112}$ Reproduced with permission from Zhang et al., "Thermal characterization and simulation study of 2.5D packages with multi-chip module on through silicon interposer," in 15th IEEE Electronics Packaging Technology Conference (EPTC) (2013). Copyright 2013 Institute of Electrical and Electronics Engineers. 
onto the organic substrate of $31 \mathrm{~mm} \times 31 \mathrm{~mm} \times 1 \mathrm{~mm}$. For the molded packages, additional molding process is conducted on the wafer level to form the epoxy molded encapsulation on the chip package. The molding layer helps protect the die from damage such as mechanical shock. The wafer is then diced into the package size. After the BGA solder ball attach process, the package is assembled on the thermal test board for electrical connections.

For Theta JA test, the test board is arranged in the one feet cubic chamber conforming to the JEDEC standard. ${ }^{121}$ The corresponding junction to ambient thermal resistances is plotted in Figure 68 as against the power input.

In Theta JA and Theta JB measurements and corresponding thermal simulation that will be discussed in the later paragraph, the molded packages give consistently lower thermal resistances than the bare die packages. This can be attributed to the enhanced thermal radiation effect due to the molding compound with the surface emissivity $\sim 0.9$, which is larger than that of $\mathrm{Si}$ surfaces in the range of 0.1 for the bare die package. Molding on the substrate also assists the heat to be conducted away from the die to the substrate. The thermal models are constructed for the molded packages and the results are compared in Figure 69. Thermal radiation effect is considered in the Theta JA thermal simulation.

The validated thermal model can also be used to predict the maximum power dissipation from the simultaneous heating of TV1 and TV2 dies under passive cooling condition. This can be achieved by assigning power dissipations of $1 \mathrm{~W}$ and $2 \mathrm{~W}$ on the two dies while keeping the thermal die power to be zero. The interaction of the multi chip heating on the $1 \mathrm{~s} 0 \mathrm{p}$ thermal board under natural convection condition can be expressed in the linear superposition form, namely,

$$
\left[\begin{array}{l}
\Delta T_{j 1} \\
\Delta T_{j 2}
\end{array}\right]=\left[\begin{array}{ll}
A 11 & A 12 \\
A 21 & A 22
\end{array}\right]\left[\begin{array}{l}
P 1 \\
P 2
\end{array}\right]=\left[\begin{array}{ll}
33.0 & 19.0 \\
22.2 & 28.9
\end{array}\right]\left[\begin{array}{l}
P 1 \\
P 2
\end{array}\right],
$$

where $\Delta \mathrm{T}_{\mathrm{j} 1}$ and $\Delta \mathrm{T}_{\mathrm{j} 2}$ are the junction temperature rises in ${ }^{\circ} \mathrm{C}, \mathrm{A}_{11}, \mathrm{~A}_{12}, \mathrm{~A}_{21}$, and $\mathrm{A}_{22}$ are the influence factors in ${ }^{\circ} \mathrm{C} / \mathrm{W}$

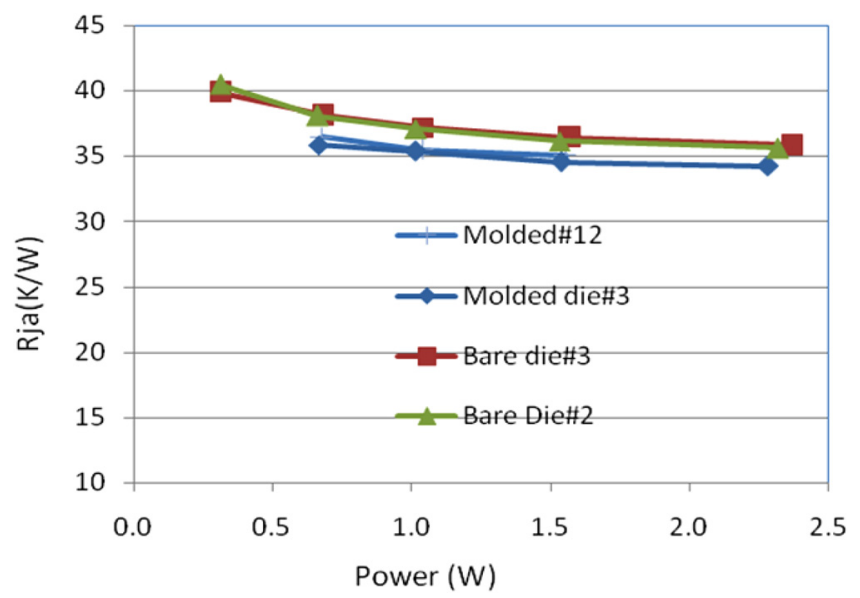

FIG. 68. Measured Theta JA test jig under natural convection condition for both molded and bare die package. ${ }^{112}$ Reproduced with permission from Zhang et al., "Thermal characterization and simulation study of $2.5 \mathrm{D}$ packages with multi-chip module on through silicon interposer," in 15th IEEE Electronics Packaging Technology Conference (EPTC) (2013). Copyright 2013 Institute of Electrical and Electronics Engineers.

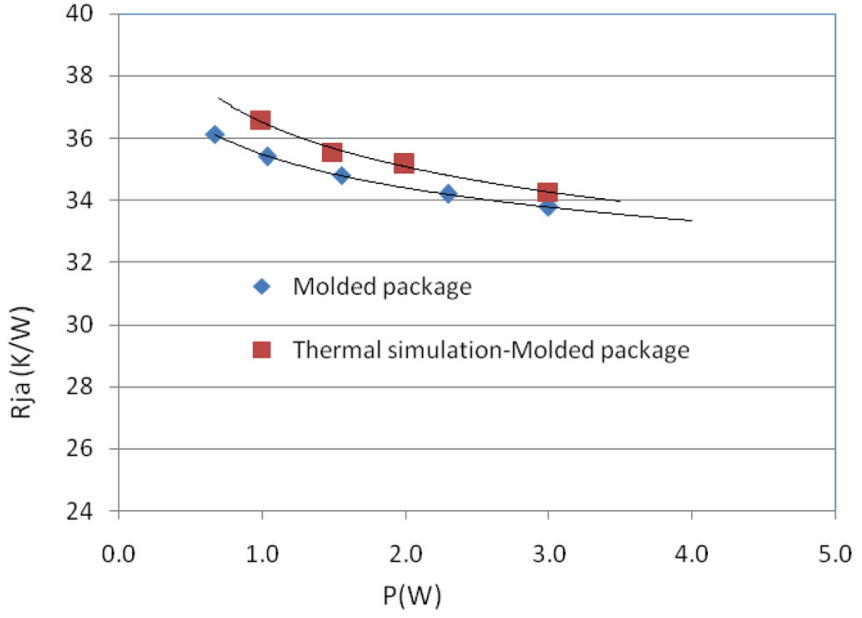

FIG. 69. Comparison of averaged simulation results versus measurements for the junction to ambient thermal resistances under natural convection condition. ${ }^{112}$ Reproduced with permission from Zhang et al., "Thermal characterization and simulation study of 2.5D packages with multi-chip module on through silicon interposer," in 15th IEEE Electronics Packaging Technology Conference (EPTC) (2013). Copyright 2013 Institute of Electrical and Electronics Engineers.

due to the power dissipations P1 and P2 in Watts for TV1 and TV2, respectively. With the above equation, the present package format could dissipate $2.3 \mathrm{~W}$ at the temperature rise of $\Delta \mathrm{T}_{\mathrm{j} 1}, \Delta \mathrm{T}_{\mathrm{j} 2} \sim 60^{\circ} \mathrm{C}$. To improve the power dissipation without heat sink on top, an immediate method is to use a thermally enhanced 4 layer PCB $(2 s 2 p)$. The computed power dissipation from a $4 \mathrm{~L}$ PCB is correlated with the linear superposition approach through thermal simulation. The power dissipation with the two chips on TSI package can go up to $3.5 \mathrm{~W}$ with the same temperature window of $60^{\circ} \mathrm{C}$.

\section{Active cooling}

\section{Active air cooling}

Typical active cooling involves either fan cooled air cooling or pump-driven liquid cooling technology. Fan cooled air technology assembled with heat sink is commonly used to manage the heat dissipation of high power chips such as processors and graphic chips. Such combination is able to remove heat in the range of $50 \mathrm{~W} / \mathrm{cm}^{2}$. Enhanced air cooling technique such as vapor chamber based heat sink can be utilized to minimize the heat spreading resistance from the small chips to the large heat sink base. For a 3D package, due to the interlayer thermal resistance, the cooling capacity for the stacked chips is limited. Additional thermal resistance is found for the bottom chip, which is mostly a logic die. A study on the shaped lid for cooling of memory dies stacked on logic die showed that the junction to casing thermal resistance is almost doubled with the use of heat sink at top, ${ }^{113}$ which may limit the performance of the logic die. On the other hand, inter layer cooling seems to be the only way to follow trend of the $3 \mathrm{D}$ high power integration.

\section{Liquid cooling}

Liquid cooling has a much higher heat transport capability than air cooling. Efforts have been dedicated to develop 
liquid cooling in the last decade from flip-chip packages to 3D packages. Chen et al. conducted the direct cooling with dielectric fluid such as FC-72 for the direct cooling of stacked MCM as shown in Figure 70. ${ }^{106}$ Using minimum flow clearances and maximum flowrate of $25 \mathrm{~g} / \mathrm{s}$, the maximum temperature reduces to $31^{\circ} \mathrm{C}$ for total power input of $6 \mathrm{~W}$, which is equivalent to a total power input of $23 \mathrm{~W}$ if the maximum temperature is increased to $56^{\circ} \mathrm{C}$ under the single phase cooling regime. Further experimental work was conducted and more than $40 \mathrm{~W}$ power can be dissipated from the $3 \mathrm{D}$ stack to maintain the junction temperature below $85^{\circ} \mathrm{C}$.

Integration of interlayer cooling on $3 \mathrm{D}$ package has received increasing interests. The technology challenges mainly lie in the design and implementation of electrical TSVs, thermal TSVs, fluidic TSVs, or any permutations of the three. As a result, the interlayer cooling of 3D stacked MCM through fluidic and electrical vias ought to be examined. The 3D package consists of two carriers assembled one over another with a Si spacer. The Si spacer is designed with fluidic and electrical interconnects. Both the carriers mounted with a $10 \mathrm{~mm} \times 10 \mathrm{~mm}$ chip having heating and temperature sensing elements. The carrier is built in with microchannels for heat transfer enhancement and extracted the heat generated by the chip. The stacked module was attached to a printed circuit board using $300-\mu \mathrm{m}$ solder balls. The footprint of the carrier is $15 \mathrm{~mm} \times 15 \mathrm{~mm} \times 0.8-\mathrm{mm}$. The liquid cooling solution consists of accessory thermal components in addition to the package: a mini-pump, an adapter for flow distribution, and a mini heat exchanger. A schematic of the cooling solution is shown in both Figures 71 and 72. Electrical and fluidic connectivity between carriers were achieved by TSV in the spacer. The cooling liquid is circulated through the carrier, and the heat from the chip is transported to the heat exchanger.

The entropy generation related to the pressure drop and heat transfer are utilized as a selection parameter $S^{*}$, given by Eqs. (5.7). The $\mathrm{S}^{*}$ for different micro-channel spacing is obtained, indicating micro-channel width from $80 \mu \mathrm{m}$ to $110 \mu \mathrm{m}$ having better results for various channel depth considered in our design. A micro-channel size of $100-\mu \mathrm{m}$ width and 350- $\mu \mathrm{m}$ depth was chosen finally for the test vehicle fabrication considering the low entropy generation and ease of fabrication

$$
\begin{aligned}
& S^{*}=S_{\text {thermal }} \times S_{d P}, \\
& S_{\text {thermal }}=\left(\frac{P}{T_{\text {ref }}}\right)^{2},
\end{aligned}
$$

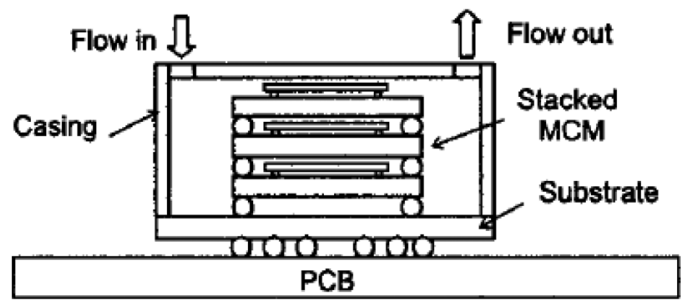

FIG. 70. Schematic of the direct cooling of 3D stacked MCM.

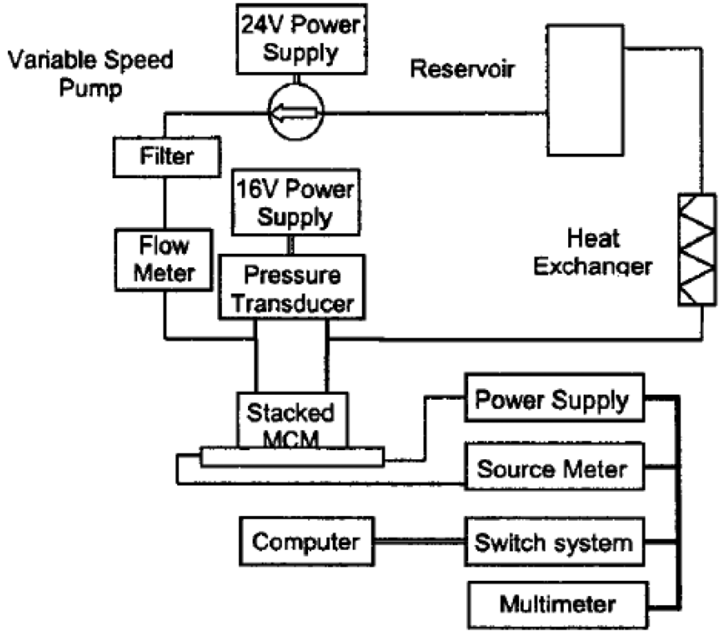

FIG. 71. Schematic of liquid cooling characterization.

$$
S_{d P}=\left(\frac{d P \times G}{T_{r e f}}\right) .
$$

A common micro-channel arrangement is single inlet and single outlet arrangement (S1). The pressure drop across a channel is, however, high due to the long flow length, which also leads to higher chip temperature at the downstream. Due to this drawback, a split flow arrangement with dual in-ports is also designed. A schematic of the single in-port and dual in-ports design is shown in Figure 73. D1 is having a constant supply plenum, and D2 is having a reducing supply plenum. This arrangement has the benefit of reduced flow length, with the thermal developing length being a substantial portion of the channel length. We evaluated two types of dual-port design and show results in Table VIII and Figure 74 based on thermal modeling. The channel wall (fin) thickness of $50 \mu \mathrm{m}$, the channel width of $100 \mu \mathrm{m}$, and the channel depth of $350 \mu \mathrm{m}$ were used for the heat transfer analysis. The inlet temperature of the water was assumed to be $50^{\circ} \mathrm{C}$, and a uniform heat load of $100 \mathrm{~W} / \mathrm{cm}^{2}$ was applied. By comparing the single-port and the dual-port designs, it is clear that the dual-port design has lower thermal resistance. The design

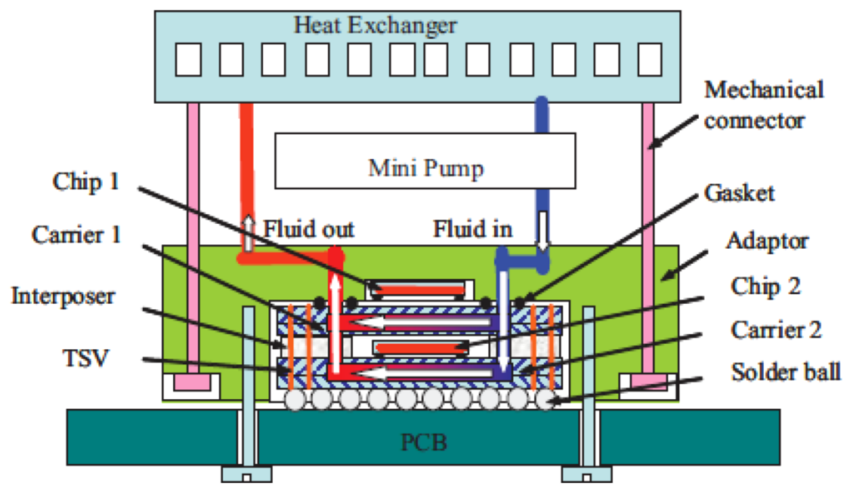

FIG. 72. Scheme of the integrated liquid cooling system for 3D stacked module (no to the scale). ${ }^{108}$ Reproduced with permission from Khan et al., "3-D packaging with through-silicon via (TSV) for electrical and fluidic interconnections," IEEE Trans. Compon., Packag., Manuf. Technol. 3(2), 221 (2013). Copyright 2013 Institute of Electrical and Electronics Engineers. 


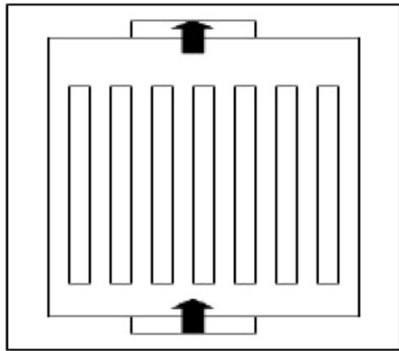

(a)

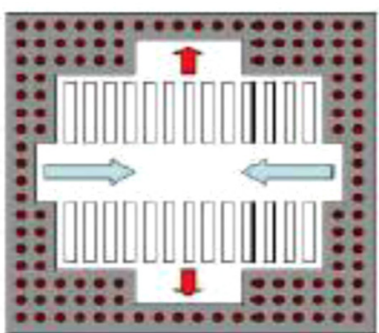

(b)

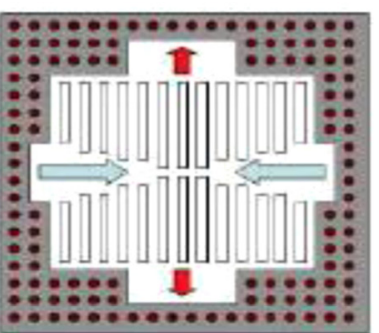

(c)
FIG. 73. (a) Single port carrier design S1 and dual port carrier designs, (b) D1, and (c) D2 on the right hand side. ${ }^{108}$ Reproduced with permission from Khan et al., "3-D packaging with through-silicon via (TSV) for electrical and fluidic interconnections," IEEE Trans. Compon., Packag., Manuf. Technol. 3(2), 221 (2013). Copyright 2013 Institute of Electrical and Electronics Engineers.
S1 has uniform flow distribution, but the long channel length results in large temperature gradient. Comparing D1 and D2, design D2 has uniform flow distributions through the microchannels and lower thermal resistance (based on peak chip temperature) and smaller temperature variation within the chip. Hence, we have selected D2 for the test vehicle fabrication and thermal characterization.

The developed thermal solution for the 3D stack MCM is shown in Figure 75. The measured thermal resistance is show in Figure 76. Up to $100 \mathrm{~W} / \mathrm{cm}^{2}$ power dissipation is tested based on the present micro-channel design (D2), with a measured thermal resistance of $0.577 \mathrm{kcm}^{2} / \mathrm{W}$. This value is $40 \%$ higher than the simulation result, which could be attributed to the non-uniform flow distribution across the two carriers.

The 2.5D package is viewed as the most viable technology for 3D integration. One of the key reasons is that conventional liquid cooling techniques such micro-channels and metallic foam heat sinks ${ }^{104,105}$ could be applied directly since a $2.5 \mathrm{D}$ package is essentially planar, compatible with the interposer platform. In this section, the liquid cooling for a $2.5 \mathrm{D}$ package shown in Sec. V B is demonstrated. A liquid cooling loop is established, which includes the micro pump (Monarch pump), heat exchanger (Lytron LL510), temperature controlled chiller (Huber K6S-NR), together with the piping and fittings. The coolant water temperature is set to $24{ }^{\circ} \mathrm{C}$, which is within $1{ }^{\circ} \mathrm{C}$ variation from the ambient temperature. The exact inlet temperature is measured with a J-type thermocouple mounted at fluid in-port. The flow rate is fixed to be $0.4 \mathrm{ml} / \mathrm{min}$ throughout the experiments. A mini channel heat sink made of copper plated with $\mathrm{Ni}$ is utilized to remove the heat from the chip top, as is shown in Figure 77.

TABLE VIII. Thermal simulation results for the different carrier designs. ${ }^{108}$ Reproduced with permission from Khan et al., "3-D packaging with through-silicon via (TSV) for electrical and fluidic interconnections," IEEE Trans. Compon., Packag., Manuf. Technol. 3(2), 221 (2013). Copyright 2013 Institute of Electrical and Electronics Engineers.

\begin{tabular}{lcccc}
\hline \hline $\begin{array}{l}\text { Flow rate } \\
(\mathrm{ml} / \mathrm{min})\end{array}$ & Design & $\begin{array}{c}\text { Pressure } \\
\text { drop }(\mathrm{mbar})\end{array}$ & $\begin{array}{c}\text { Maximum } \\
\text { temperature }\left({ }^{\circ} \mathrm{C}\right)\end{array}$ & $\begin{array}{c}\text { Temperature } \\
\text { variation }\left({ }^{\circ} \mathrm{C}\right)\end{array}$ \\
\hline 100 & S1 & 158.1 & 98.7 & 22.5 \\
& D1 & 55.45 & 97.2 & 8.6 \\
& D2 & 76.7 & 93.4 & 9.4 \\
200 & S1 & 398.7 & 92.1 & 18.5 \\
& D1 & 169.7 & 91.8 & 9.6 \\
& D2 & 253.1 & 87.2 & 7.8 \\
\hline \hline
\end{tabular}

The measured junction to heat sink thermal resistances (Theta JC) is tabulated in Table IX. It is shown that the Theta JC for the bare die package is one order of magnitude smaller than the molded package. The molding layer, with a thermal conductivity of $0.7 \mathrm{~W} / \mathrm{mK}$, unfavorably adds to the thermal resistance. For the bare die package, larger power inputs $(8-20 \mathrm{~W})$ are supplied to achieve a junction temperature rise $7-17^{\circ} \mathrm{C}$ to minimize junction temperature error in the Theta JC data reduction. A common problem in Theta JC measurement for the bare die package is the misalignment of the cold plate base with the top of the test die. Misalignment would generate additional interfacial thermal resistance and abnormally high on-chip temperature variation.

The simulation model is also conducted and the corresponding heat flux and temperature profiles for the molded package are shown in Figure 78. Since the thermal performance of the mini channel heat sink is not known, the detailed fin/channel structure is modeled together with the inlet and outlet to extract the thermal performance. A comparison of computed and measured Theta JC has been shown in Table IX. Good agreement is found for the bare die package. For the molded package, a relatively obvious discrepancy of $10.4 \%$ is found. The molded package is cross-sectioned, and the microscopic view shows a surface roughness approximately $15-20 \mu \mathrm{m}$. The presence of roughness is beneficial to the mold release process, but it may cause additional

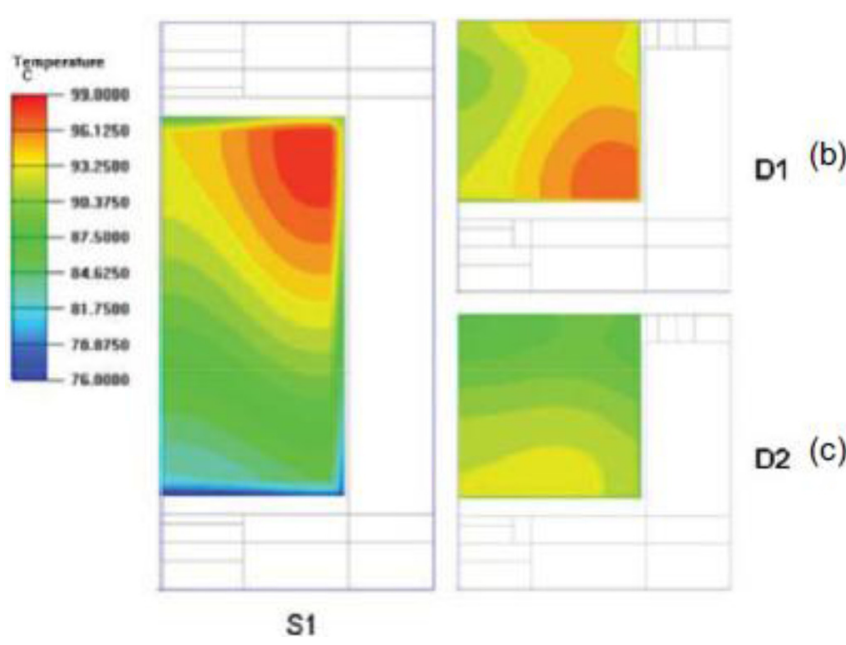

FIG. 74. Single port carrier design (S1) and dual port carrier designs (D1 and D2). ${ }^{108}$ Reproduced with permission from Khan et al., "3-D packaging with through-silicon via (TSV) for electrical and fluidic interconnections," IEEE Trans. Compon., Packag., Manuf. Technol. 3(2), 221 (2013). Copyright 2013 Institute of Electrical and Electronics Engineers. 


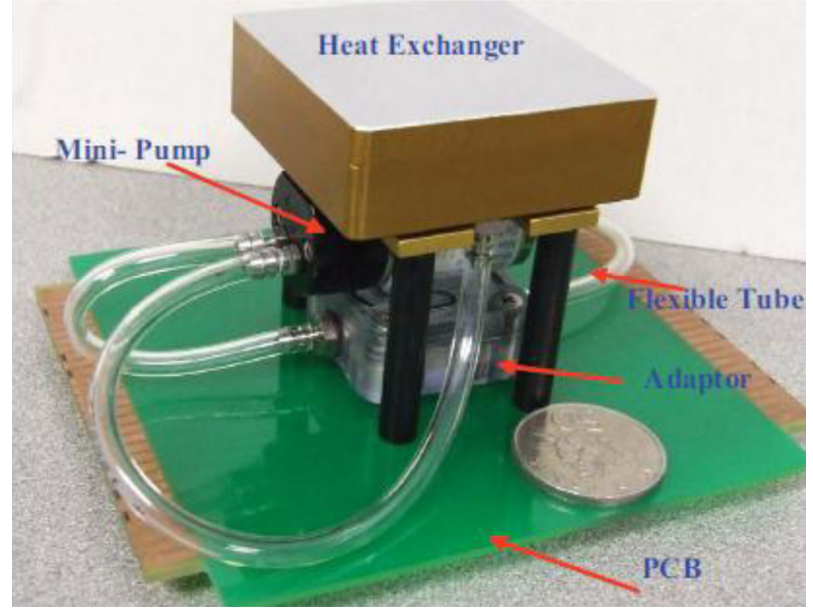

FIG. 75. Photograph of integrated cooling. ${ }^{108}$ Reproduced with permission from Khan et al., "3-D packaging with through-silicon via (TSV) for electrical and fluidic interconnections," IEEE Trans. Compon., Packag., Manuf. Technol. 3(2), 221 (2013). Copyright 2013 Institute of Electrical and Electronics Engineers.

interfacial thermal resistance. Surface warpage also contributes to the deviation of simulation from measurement. Surface polishing process may be considered to minimize the roughness on the top of the molding compound and to reduce Theta JC.

With the validated thermal model, the thickness of overmolding for the 2.5D package is examined. Figure 79 shows the Theta JC versus the molding thickness based on simulation study. It is seen that, with the increase in overmolding thickness from 0 to $0.6 \mathrm{~mm}$, the thermal resistance increases drastically from $0.82 \mathrm{~K} / \mathrm{W}$ to $11.76 \mathrm{~K} / \mathrm{W}$, mainly due to the thermal conduction resistance across the overmolding layer. If additional thermal solution such as heat sink is applied at the top of the package, a thin overmolding thickness of $\sim 0.1 \mathrm{~mm}$ or even bare die package can be applied to minimize the overall thermal resistance.

\section{Thermal aware 2.5D design and implementation}

With the growing emphasis on high performance and small form factor, and for keeping up with the advancement

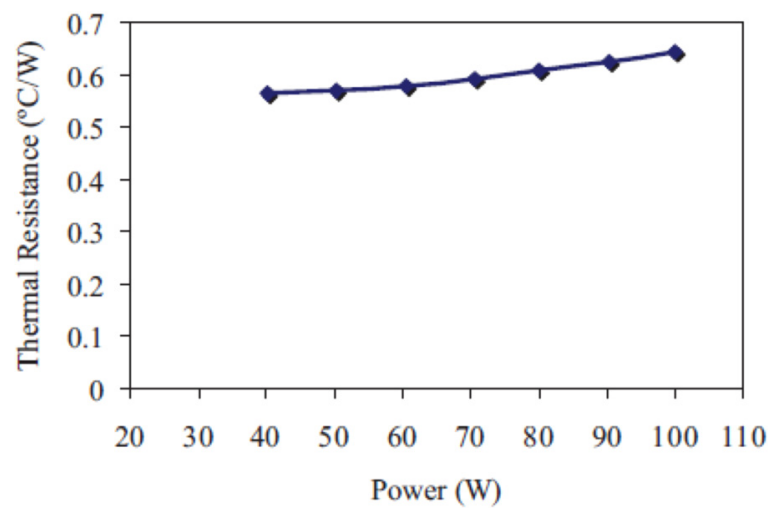

FIG. 76. Thermal resistance versus power input for the 3D package. ${ }^{108}$ Reproduced with permission from Khan et al., "3-D packaging with through-silicon via (TSV) for electrical and fluidic interconnections," IEEE Trans. Compon., Packag., Manuf. Technol. 3(2), 221 (2013). Copyright 2013 Institute of Electrical and Electronics Engineers.

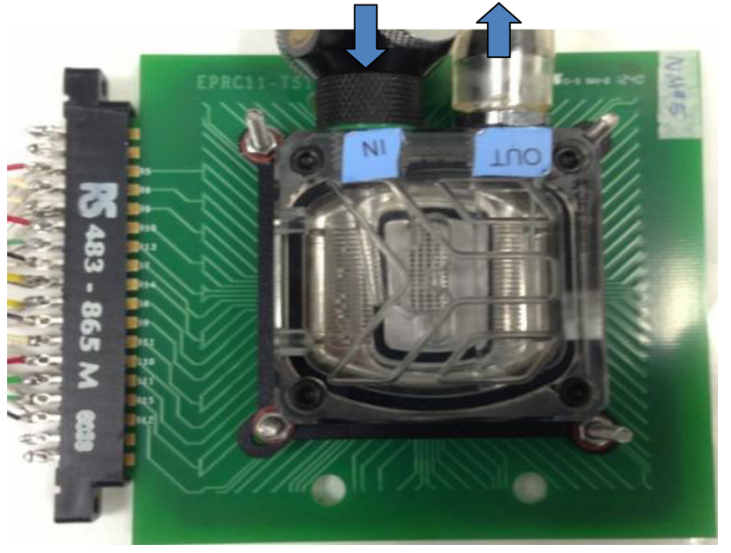

FIG. 77. Liquid cooling of 2.5D package with liquid cooling configuration.

of $2.5 \mathrm{D} / 3 \mathrm{D}$ fabrication, thermal design has been indispensable for the implementation of $2.5 \mathrm{D} / 3 \mathrm{D}$ integration. For space and energy tight applications, passive cooling is the preferred option. The use of interposer with properly designed TSVs can help reduce the junction temperature and minimize the hot spots on the chip. Design factors to consider include chip size, chip stacks, distributed chip arrangement and hot spot staggering on the chip, type of encapsulating mold compound, heat spreader, thermal bridging with thermally conductive adhesive, and so on.

For high power applications, 2.5D package benefits from existing cooling solutions for 2D planar chips without much change on the package format. On the other hand, interlayer cooling through liquid cooling would become the trend for the stacked high power chips and modules. Microchannels or micro pins can be used as the thermal enhancement structures. The cooling geometry, combined with TSVs and micro-bumps are influencing factors on the thermal and flow performances. Alternative direct dielectric fluid cooling as well as water based fluidic cooling can be considered. Proper liquid sealing methods are required to avoid liquid spilling, evaporation loss and possible damage to electronic circuits. Thermoelectric coolers can also be conjectured, although it is still at early stage due to limited coefficient of performance (COP). CMOS compatible Silicon Nanowires (SiNWs) fabricated on-chip can be an effective means for hot spot cooling in the $2.5 \mathrm{D} / 3 \mathrm{D}$ packages, as well as for temperature control. Increase in nanowire number, reduction of thermal conductivity and contact resistance will lead to enhanced cooling capacity.

Thermal aware design is becoming of increasing importance. Thermal simulation shall be utilized in the design stage to examine the on-chip power dissipation and hot spot distribution arising from electrical design, the effects of package formats and material parameters, and the cooling

TABLE IX. Measured and simulation results for Theta JC.

\begin{tabular}{lcccc}
\hline \hline Package types & $\begin{array}{c}\text { Power } \\
\text { input }(\mathrm{W})\end{array}$ & $\begin{array}{c}\text { Measured } \\
\mathrm{R}_{\mathrm{JC}}(\mathrm{K} / \mathrm{W})\end{array}$ & $\begin{array}{c}\text { Simulation } \\
\mathrm{R}_{\mathrm{JC}}(\mathrm{KAV})\end{array}$ & $\begin{array}{c}\text { Difference from } \\
\text { measurements }(\%)\end{array}$ \\
\hline Molded package & 2 & 10.9 & 9.76 & -10.4 \\
Bare die package & 14 & 0.34 & 0.82 & -0.024 \\
\hline \hline
\end{tabular}




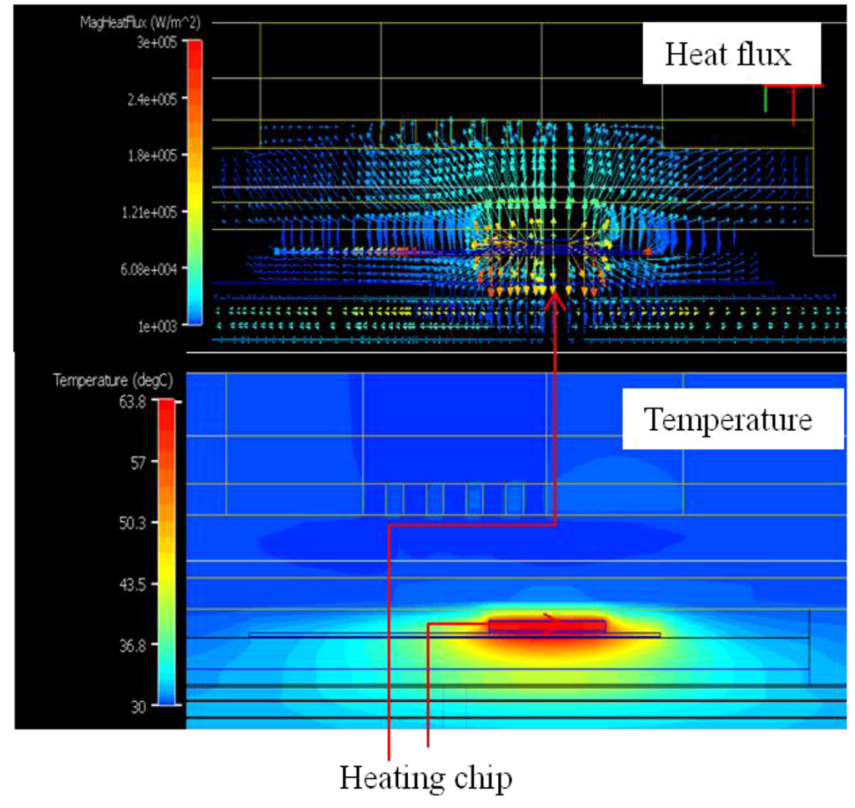

FIG. 78. Heat flux and temperature profiles for the molded package under cold plate cooling for determining the top thermal resistance.

design efficiency and the feasibility. Thermal simulation can be a useful tool in chip, TSV, and package optimization. Commercial simulation tools such as Flotherm, Icepak Comsol multi-physics are typically used for numerical modeling and optimization.

On the other hand, the thermal characterization with specially designed thermal test dies shall be used for model and design validation. In practice, the thermal simulation does not take into consideration certain complexities and tends to overestimate the thermal performance. The overestimation can be attributed to insufficient thermal and fluidic design, unanticipated packaging and process deviation. Therefore, there is a continuous need to establish the thermal characterization methodologies and develop verification cases for simulation models. If the model is validated, the designer can build on the framework to extend the thermal model for new designs and parameter studies, and to provide the optimized designs for various application scenarios. The

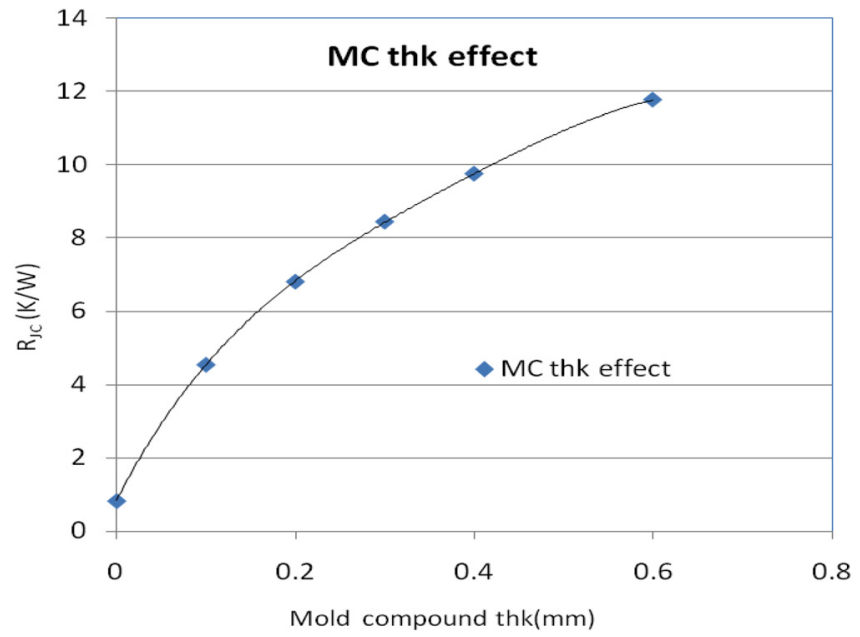

FIG. 79. Effect of molding compound thickness on the Rjc. interactive processes among electrical, thermal and package design process are illustrated in Figure 80.

\section{THERMO-MECHANICAL DESIGN AND MODELING FOR 2.5D PACKAGING}

Thermo-mechanical analysis is a critical subject that cannot be ignored when it comes to the realization of a TSI package. There are a few areas drawing the attention of TSI builders: (a) manufacturability and functionality of TSI, (b) handling of TSV wafers and chips during fabrication and assembling, and (c) packaging and long-term reliability. FEM is now a widely adopted approach for performing thermo-mechanical analysis in the industry. This section outlines the use of FEM in resolving various thermo-mechanical challenges in $2.5 \mathrm{D}$ packaging.

\section{A. Manufacturability and functionality of TSI}

The CTE of copper and Si are about 17 and $3 \mathrm{ppm} /{ }^{\circ} \mathrm{C}$, respectively. The large difference in CTE between copper and $\mathrm{Si}$ induces a significant level of stress and strain around a copper-filled TSV when it is subjected to a substantial amount of thermal loading. Figure 81 shows the deformation of a copper-filled TSV when it is subjected to a temperature change. ${ }^{123}$ During a temperature decrement, the copper inside the via contracts much more than the Si which holds it. The surrounding Si thus experiences, in general, a state of tension in both the radial and circumferential directions and at the same time compression in the axial direction. For the case of a TSI which comprises an array of TSVs, the stress field on the $\mathrm{Si}$ is a combined result of individual stress fields around each TSV.

Mechanical failures and alteration in electrical performance are adverse outcomes reported due to this stress field. Wafer cracking and delamination are common mechanical failure modes that could be encountered during the fabrication of TSI. There are design guidelines generated by FEM and we shall discuss them in Subsections VI A 1-VI A 3. The large piezoresistivity of doped Si makes active devices like p-MOSFET and n-MOSFET susceptible to variations in their electrical performance when under the influence of TSVs. The placement of these devices, thus, requires careful

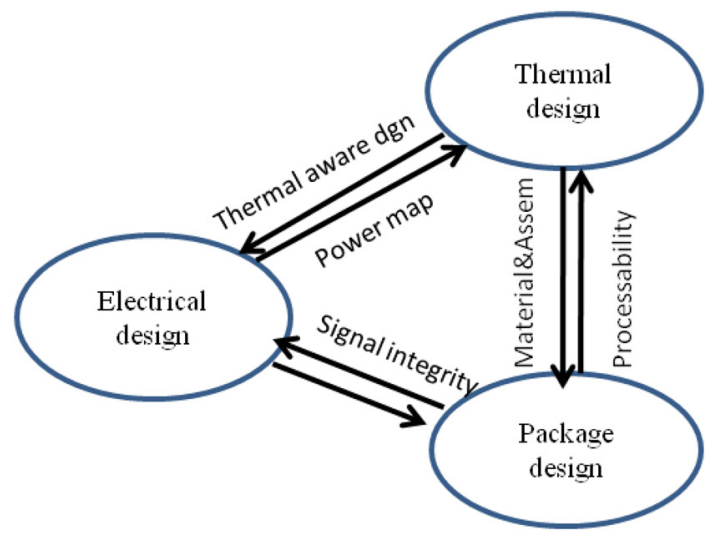

FIG. 80. Interaction among electrical, thermal design, and package design for technology implementation. 


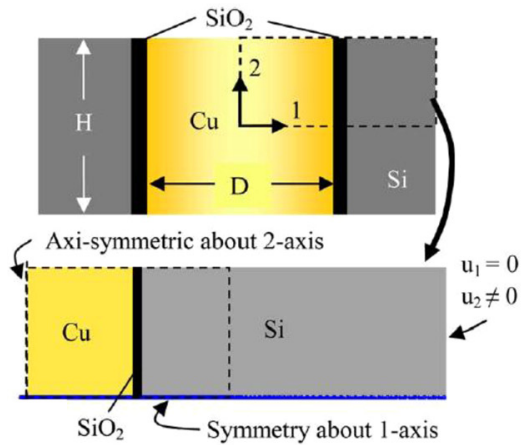

(a)

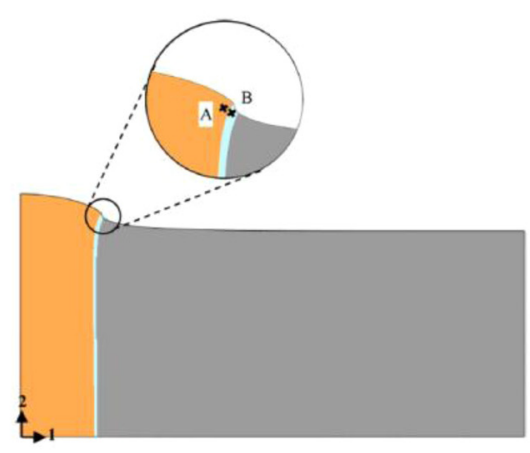

(b)

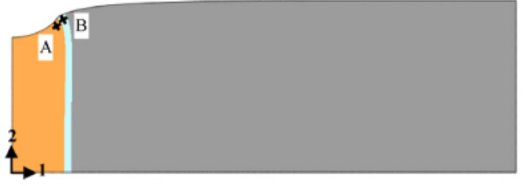

(c)

FIG. 81. Modeling of a copper-filled TSV (a) modeling strategy, (b) deformation of via during a temperature increment, and (c) deformation of via during a temperature decrement. ${ }^{123}$ Reproduced with permission from Selvanayagam et al., "Nonlinear thermal stress/strain analyses of copper filled TSV (through silicon via) and their flip-chip microbumps," IEEE Trans. Adv. Packag. 32(4), 720 (2009). Copyright 2009 Institute of Electrical and Electronics Engineers.

considerations. These aspects will be illustrated by the example below.

\section{Design rules for avoiding wafer cracking}

Wafer cracking can occur after the annealing process when the annealing temperature is too high and/or the TSVS are too tightly packed. ${ }^{124}$ Accordingly, the chance of this failure can be characterized by the 1 st principal stress $\sigma_{1}$ induced on the chip surface at the edge of a TSV as depicted in Figure 82.

Modeling results suggest that the chance of wafer cracking increases with larger TSV diameter, smaller TSV pitch-to-diameter ratio, smaller TSV depth, smaller dielectric layer thickness, smaller barrier layer thickness, larger wafer thickness, and higher annealing temperature. The minimum required pitch-to-diameter ratio $p: d$ for fabricating a TSI at a given annealing temperature $T$ may be estimated empirically by ${ }^{124}$

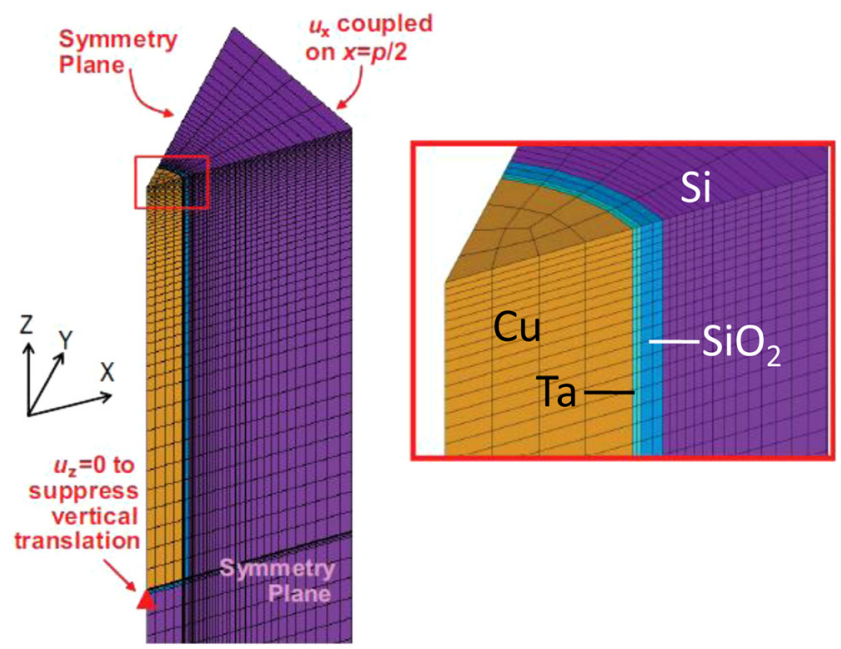

(a)

$$
\begin{aligned}
& \eta \cdot k \cdot\left[1+2.76\left(\frac{2 p}{d}-1\right)^{-2}\right] \leq 287 \mathrm{MPa}, \\
& \text { where } k=\frac{E_{\mathrm{via}}\left(\alpha_{\mathrm{via}}-\alpha_{\mathrm{si}}\right)\left(T-25^{\circ} \mathrm{C}\right)}{1-2 \nu_{\mathrm{via}}+\frac{1+\nu_{\mathrm{si}}}{1+\nu_{\mathrm{via}}} \frac{E_{\mathrm{via}}}{E_{\mathrm{si}}}}, \\
& E_{\mathrm{via}}=f_{\mathrm{Cu}} E_{\mathrm{Cu}}+f_{\mathrm{SiO}_{2}} E_{\mathrm{SiO}_{2}}+f_{\mathrm{Ta}} E_{\mathrm{Ta}}, \\
& \nu_{\mathrm{via}}=f_{\mathrm{Cu}} \nu_{\mathrm{Cu}}+f_{\mathrm{SiO}_{2}} \nu_{\mathrm{SiO}_{2}}+f_{\mathrm{Ta}} \nu_{\mathrm{Ta}}, \\
& \alpha_{\mathrm{via}}=\frac{f_{\mathrm{Cu}} E_{\mathrm{Cu}} \alpha_{\mathrm{Cu}}+f_{\mathrm{SiO}_{2}} E_{\mathrm{SiO}_{2}} \alpha_{\mathrm{SiO}_{2}}+f_{\mathrm{Ta}} E_{\mathrm{Ta}} \alpha_{\mathrm{Ta}}}{f_{\mathrm{Cu}} E_{\mathrm{Cu}}+f_{\mathrm{SiO}_{2}} E_{\mathrm{SiO}_{2}}+f_{\mathrm{Ta}} E_{\mathrm{Ta}}} .
\end{aligned}
$$

Here, $f$ is the volume fraction of the corresponding component of a TSV and $\eta$ is the safety factor advised to be above 1.1. $E, \nu$, and $\alpha$ are the Young's modulus, Poisson's ratio, and CTE of the corresponding components, respectively.

\section{Design rules for reducing copper protrusion}

Copper protrusion is one major factor causing delamination of BEOL metal layers. ${ }^{125}$ Generated by FEM, Figure 83

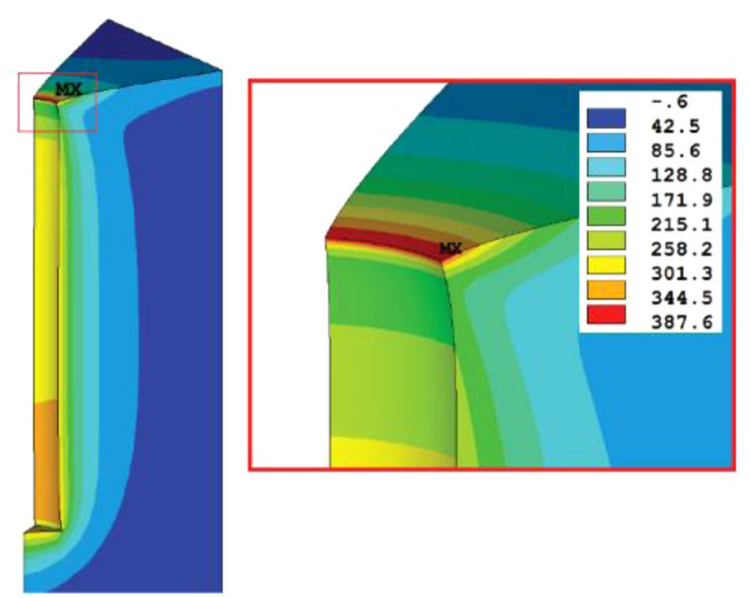

(b)

FIG. 82. Modeling of wafer cracking stress induced around a TSV. (a) Finite element model for the analysis and (b) $\sigma_{1}$ (in MPa) induced in Si. ${ }^{124} \mathrm{Reproduced}$ with permission from Chan et al., "Thermo-mechanical design rules for the fabrication of TSV interposers," IEEE Trans. Compon., Packag., Manuf. Technol. 3(4), 633 (2013). Copyright 2013 Institute of Electrical and Electronics Engineers. 

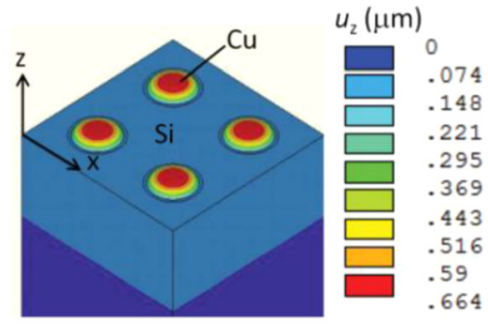

(a)

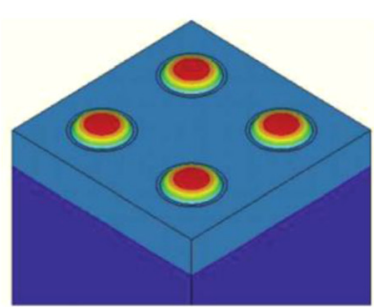

(b)

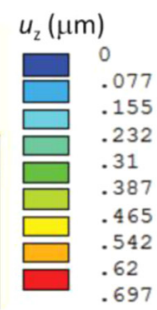

697

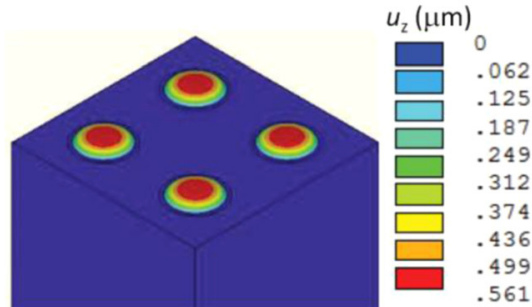

(c)

FIG. 83. Copper protrusion FEM results: (a) Right after ramping up to $400{ }^{\circ} \mathrm{C}$, (b) held for 30 min at $400{ }^{\circ} \mathrm{C}$, and (c) cooled down to room temperature. ${ }^{125}$ Reproduced with permission from Che et al., "Study on Cu protrusion of through-silicon via," IEEE Trans. Compon., Packag., Manuf. Technol. 3(5), 732 (2013). Copyright 2013 Institute of Electrical and Electronics Engineers.

illustrates the development of copper protrusion at different stages of an annealing process. Accordingly, copper protrusion grows with higher annealing temperature, longer duration, greater TSV depth, and larger TSV diameter. Hence, smaller and sparsely allocated TSVs are recommended to be adopted for reducing copper protrusion. ${ }^{125}$

As the major manufacturability concerns are raised by the via-filling material copper, there are investigations reported on the use of unfilled TSVs ${ }^{123}$ and polymer filled vias. ${ }^{126}$ These alternative technologies generally do help to reduce the thermal stress induced at the TSVs. However, they are either incompatible with the current CMOS technologies or are not in favor of the miniaturization trend. Copper, hence, remains the most popular filling material due to its compatibility and high electrical conductivity.

\section{Design for reducing mobility change of MOSFETs near TSVs}

p-MOSFET and n-MOSFET behave differently under the influence of stress. Specifically, their resistances vary to different extents with respect to different stress components. When a p-MOSFET is oriented along the [110] direction and is placed in between TSVs which are formed along the $\langle 110\rangle$ directions in a rectangular manner, it is found to be more susceptible to mobility change than an n-MOSFET no matter how the n-MOSFET is oriented. This scenario is demonstrated in Figure $84 .{ }^{127}$ At these positions, placement of

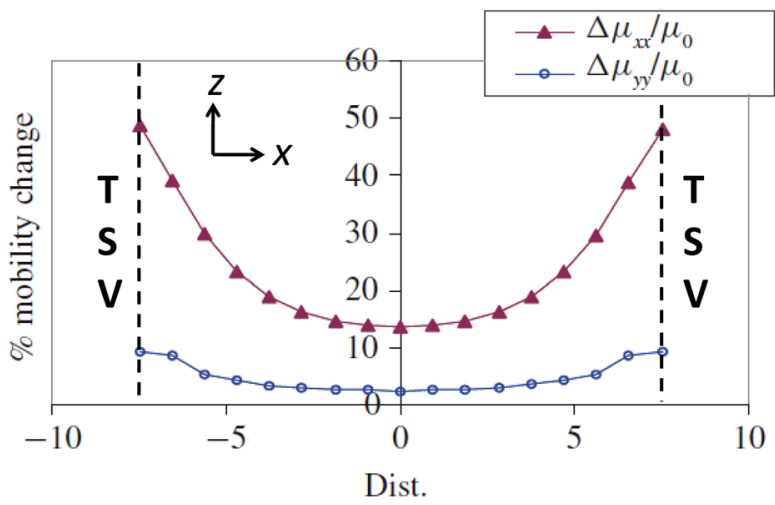

(a)
p-MOSFETs should in fact be avoided. N-MOSFETs oriented along [110] are better devices to be placed at these locations since their mobility change will be negligible $(<1 \%)$. Active devices, therefore, should be allocated strategically so as to minimize their change in electrical performance when they are placed near the TSVs.

\section{B. Handling of TSV wafers and chips during fabrication and assembling}

Warpage is a key challenge to be addressed for handling of TSV wafers. This is again a CTE mismatch problem but more packaging materials can be involved in this case. Wafer warpage can obstruct wafers from being handled properly by fabrication tools. Not only it affects fabrication accuracy but may also detrimental to the entire fabrication process. The modeling of wafer warpage is critical in $2.5 \mathrm{D}$ packaging and it will be discussed in the following subsession. The use of TSI involves at least one chip-stacking step. When a chip is to be stacked on one another, the bottom one may crack as a result of the stacking force. An example will be given below with strategies provided to solve the problem.

\section{Design for reducing TSV wafer warpage}

It is not a straight forward task to model a TSV wafer, due to the enormous amount of TSVs it carries. One methodology

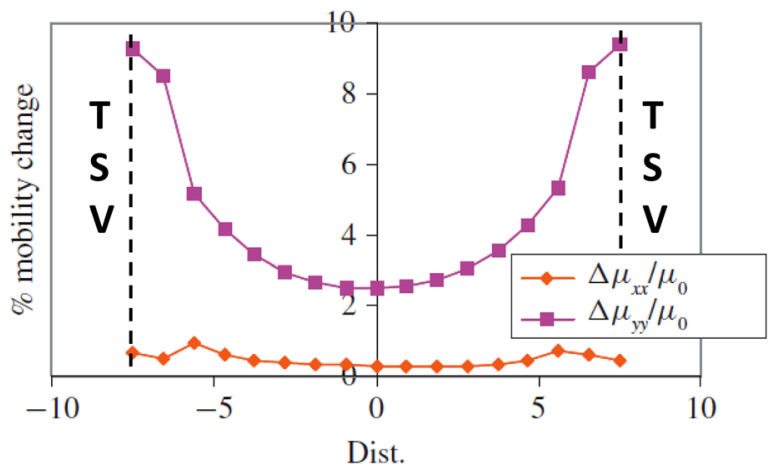

(b)

FIG. 84. Percent change in mobility due to the presence of $10 \mu \mathrm{m}$ TSVs which are $25 \mu \mathrm{m}$ apart for (a) p-MOSFET and (b) n-MOSFET. TSVs align with [110]. ${ }^{127}$ Reproduced with permission from Selvanayagam et al., "Modeling stress in silicon with TSVs and its effect on mobility," IEEE Trans. Compon., Packag., Manuf. Technol. 1(9), 1328 (2011). Copyright 2011 Institute of Electrical and Electronics Engineers. 


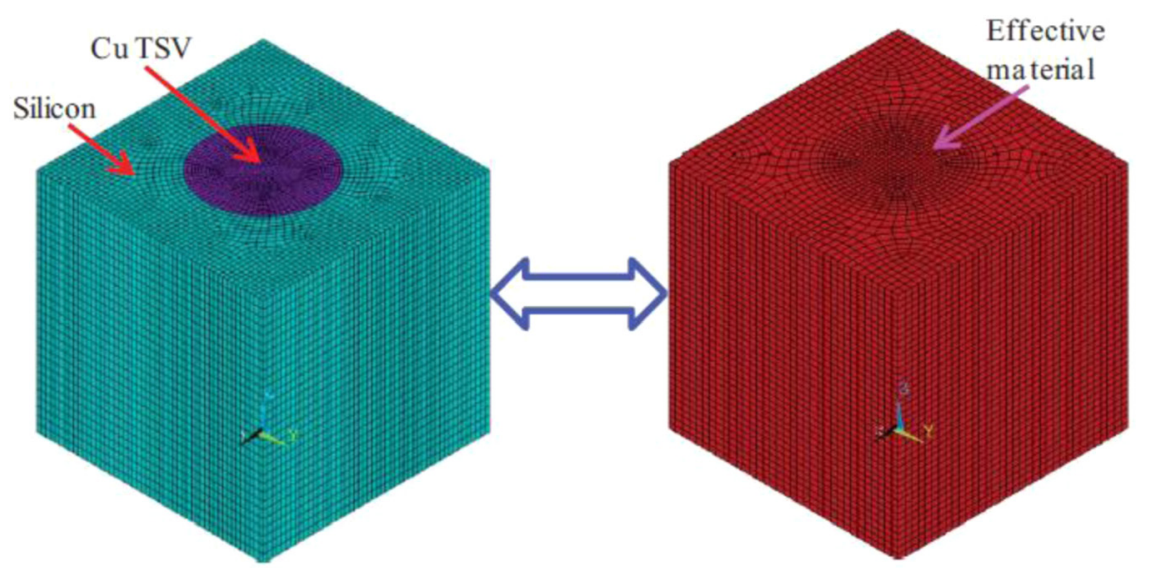

FIG. 85. Use of an effective material to develop an equivalent TSV model. ${ }^{128}$ Reproduced with permission from Che et al., "Development of wafer-level warpage and stress modeling methodology and its application in process optimization for TSV wafers," IEEE Trans. Compon., Packag., Manuf. Technol. 2(6), 944 (2012). Copyright 2012 Institute of Electrical and Electronics Engineers.

Equivalent TSV modelin one TSV unit

for achieving this mission is to adopt an "equivalent TSV model"128 as depicted in Figure 85. The model can be developed by employing an effective material such that the necessary mechanical responses of the original system can be preserved. With the equivalent TSV model, it is found that the warpage of a TSV wafer increases with larger overburden thickness, higher annealing temperature, and smaller TSV pitch-to-diameter ratio. The wafer warpage will be largest when the depth of TSVs equals one half of the wafer thickness. One effective approach for reducing the TSV wafer warpage is to introduce an extra CMP step before annealing the wafer.

\section{Design for avoiding die-cracking during chip stacking}

Die-cracking may happen to the carrier chip as it bends during a chip stacking process. ${ }^{129}$ Accordingly, the loading experienced by the carrier chip depends on the solder bump layouts of both the carrier chip and the stacking chip. Insertion of "dummy bumps" beneath the carrier chip is an effective approach for redistributing the loading exerted onto it. Figure 86 demonstrates the use of dummy bumps for reducing the deflection and bending stress experienced by a carrier chip. With the knowledge of the die strength acquired by some kinds of bending tests, the issue with die-cracking can be avoided with an optimized design with the aid of modeling.

\section{Packaging and long-term reliability}

As a result of the fragile low-k material employed today, packaging can be either protective or damaging to the chip depending on the kind of technology adopted. This area of investigation is termed as chip package interaction (CPI). TSI is, fortunately, a protective technology in terms of CPI as will be elaborated below. The long-term reliability of a package is typically characterized by its solder joint thermal fatigue performance when subjected to temperature cycling test (TCT). Critical factors in this area will be discussed as follows.

\section{CPI and micro solder bump reliability of TSI}

Figure 87 shows a typical TSI package which carries a $\mathrm{Cu}$ /low-K chip. ${ }^{130}$ If there are no TSIs, the micro-bumps will be subjected to a severe shear loading induced by the large mismatch in $\mathrm{CTE}$ between the $\mathrm{Cu} / \mathrm{low}-\mathrm{k}$ chip $\left(\sim 3 \mathrm{ppm} /{ }^{\circ} \mathrm{C}\right)$ and the bismaleimide triazine $(\mathrm{BT})$ substrate $\left(\sim 13 \mathrm{ppm} /{ }^{\circ} \mathrm{C}\right)$.
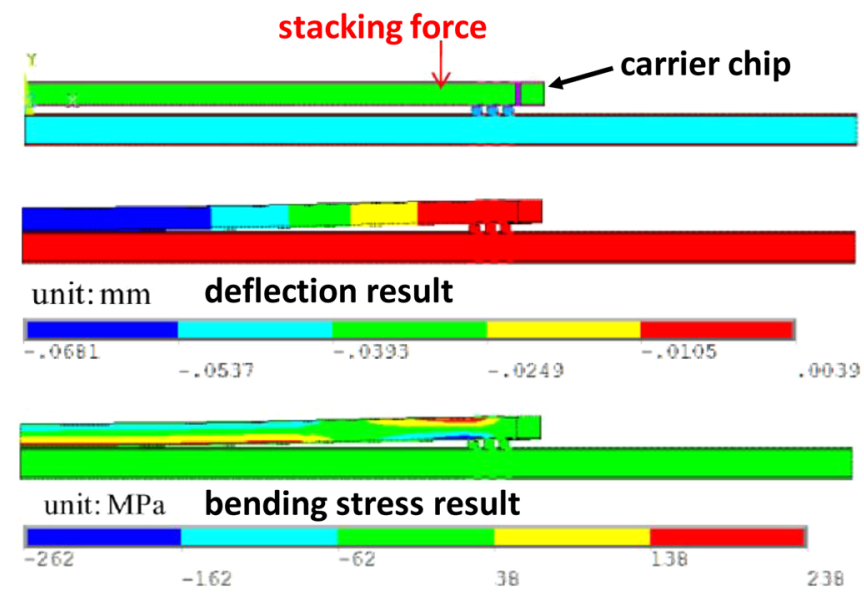

(a)
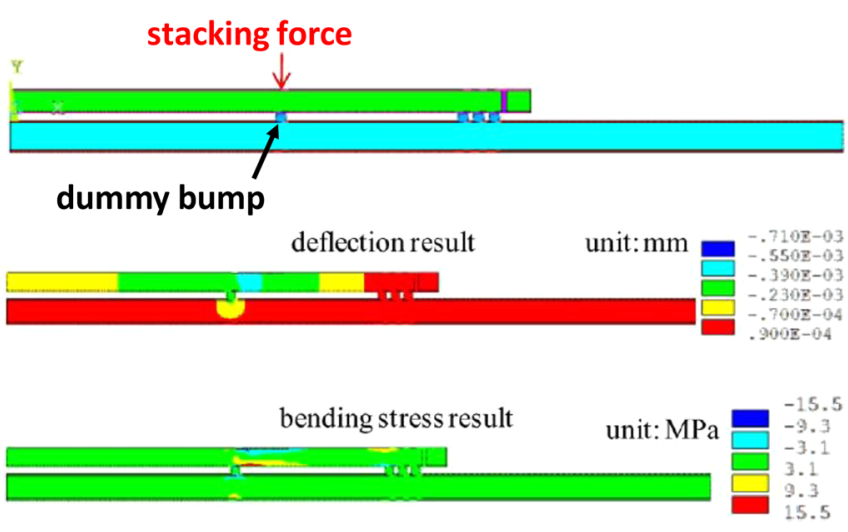

(b)

FIG. 86. Die deflection $u_{\mathrm{y}}$ and bending stress $u_{\mathrm{x}}$ results during a chip stacking process for (a) original bump layout design and (b) optimized bump layout design. ${ }^{129}$ Reproduced with permission from Che et al., "Structure design optimization and reliability analysis on a pyramidal-shape three-die-stacked package with through-silicon via," IEEE Trans. Device Mater. Reliab. 12(2), (2012). Copyright 2012 Institute of Electrical and Electronics Engineers. 


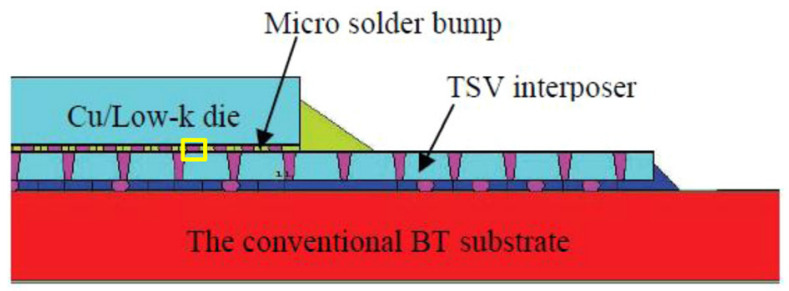

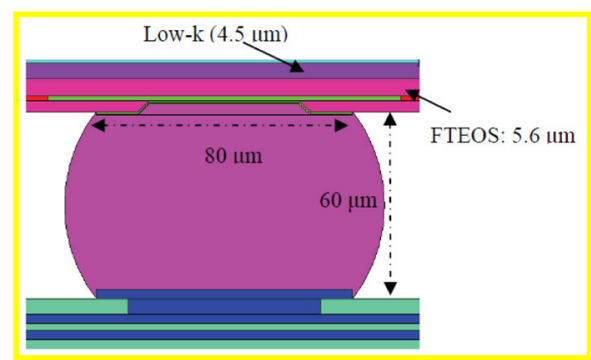

(a)

\begin{tabular}{|c|c|}
\hline $\begin{array}{c}\text { Effects of TSV } \\
\text { interposer without } \\
\text { underfill }\end{array}$ & $\begin{array}{c}\text { Max. peeling } \\
\text { stress }(S y) \text { in } \\
\text { low- } k \text { layer }(\mathrm{MPa})\end{array}$ \\
\hline $\begin{array}{c}\text { Conventional FCBGA w/o } \\
\text { TSV interposer }\end{array}$ & 250.01 \\
\hline FCBGA with TSV interposer & 125.18 \\
\hline
\end{tabular}

(b)

FIG. 87. CPI of a typical TSI package (a) Package geometry and (b) Effect of TSI on low-k stress. ${ }^{130}$ Reproduced with permission from Zhang et al., "Development of through silicon via (TSV) interposer technology for large die $(21 \times 21 \mathrm{~mm})$ fine-pitch Cu/low-k FCBGA package," in Proceedings of 59th Electronic Components and Technology Conference (EPTC), San Diego, CA, USA, May 2009, p. 305. Copyright 2009 Institute of Electrical and Electronics Engineers.

This severe shear loading will be transmitted to the low-k layer and eventually results in its damage. Now with the TSI added which possesses a CTE similar to that of a Si chip, most of the shear loading is expected to be carried by the $\mathrm{C} 4$ bumps between the TSI and the BT substrate. The use of TSI, from this perspective, is like putting a barrier (or a stress buffer layer) in between the $\mathrm{Cu} / \mathrm{low}-\mathrm{k}$ chip and the BT substrate. This helps to shield the shear loading induced to the micro-bumps and the low-k layer and hence protecting the chip. Thermo-mechanical simulation shows: (i) the impact of TSV interposer reduces the low-k stress (by more than 50\%) and correspondingly decreases the creep strain energy density per cycle $(\Delta \mathrm{W})$ in the micro solder bumps (by more than 28\%). Thus, he package reliability and micro solder joint reliability can be greatly improved, (ii) more than $60 \%$ reduction in the low-k stress and $7 \%$ reduction in $\Delta \mathrm{W}$ have been observed when the underfill between the $\mathrm{Si}$ chip and the TSV interposer, as well as the underfill between the TSV interposer and the BT substrate are used. Thus, two underfills which lead to lower stress/strain in the low-k layer and micro solder bumps are recommended, (iii) more than $50 \%$ reduction in the low-k stress and $10 \%$ reduction in the $\Delta \mathrm{W}$ in micro solder bumps have been observed when via top diameter/via bottom diameter is decreased from $100 \mu \mathrm{m} /$ $50 \mu \mathrm{m}$ to $50 \mu \mathrm{m} / 10 \mu \mathrm{m}$. Thus, a smaller via is recommended, and (iv) 59\% reduction in the low-k stress and 35\% reduction in $\Delta \mathrm{W}$ in micro solder bumps have been observed when low$\mathrm{k}$ die thickness decreases from $750 \mu \mathrm{m}$ to $300 \mu \mathrm{m}$. Thus, a thinner low-k chip is recommended.

\section{Design for enhancing solder joint reliability of packages with Si carrier}

It is understood that TSI plays a role in enhancing the solder joint reliability of micro-bumps. ${ }^{130}$ However, the $\mathrm{C} 4$ bumps that connect the TSI to the BT substrate are now more prone to failure. Underfill is the remedy to this problem, ${ }^{131}$ as shown in Figure 88. Accordingly, low CTE and high glass transition temperature $T_{\mathrm{g}}$ are favored properties for this underfill.

\section{Demonstration a low stress bond pad design for low temperature micro bumps on TSVs}

The proposed design of the pad (Figure 89) is one in which there is no direct contact between the pad and the copper-filled via. ${ }^{132,133}$ The pad is shaped such that it surrounds the via, but does not touch it. The positioning of the pad with respect to the via is such that they are coaxial. Electrical connectivity between the via and the pad is provided through the presence of traces. One possible design is

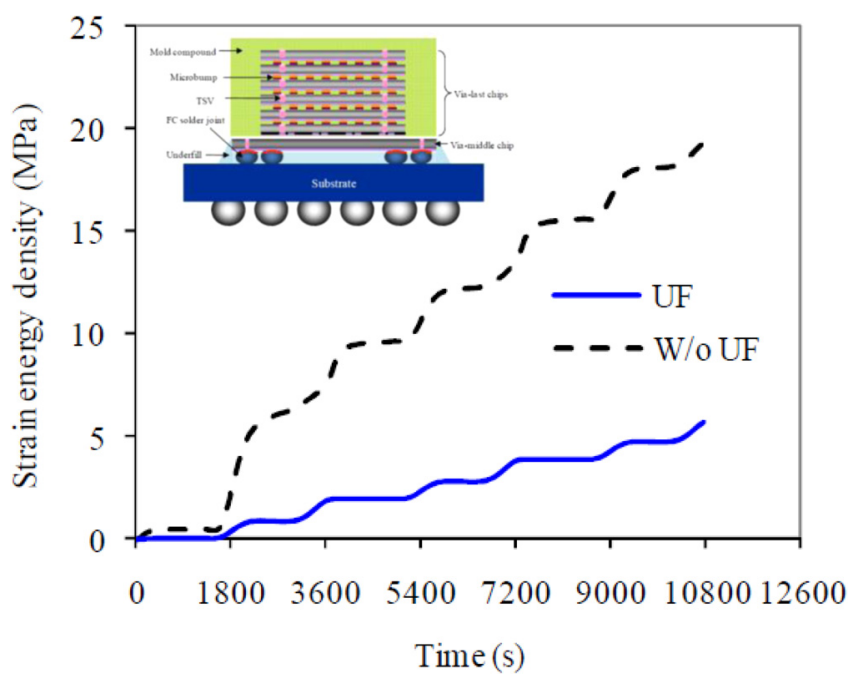

FIG. 88. Effect of underfill on C4 bumps of a 3D package. ${ }^{131}$ Reproduced with permission from Che et al., "The study of thermo-mechanical reliability for multi-layer stacked chip module with through-silicon-via (TSV)," in Proceedings of 12th Electronics Packaging Technology Conference (EPTC), Singapore, December 2010, p. 743. Copyright 2012 Institute of Electrical and Electronics Engineers. 

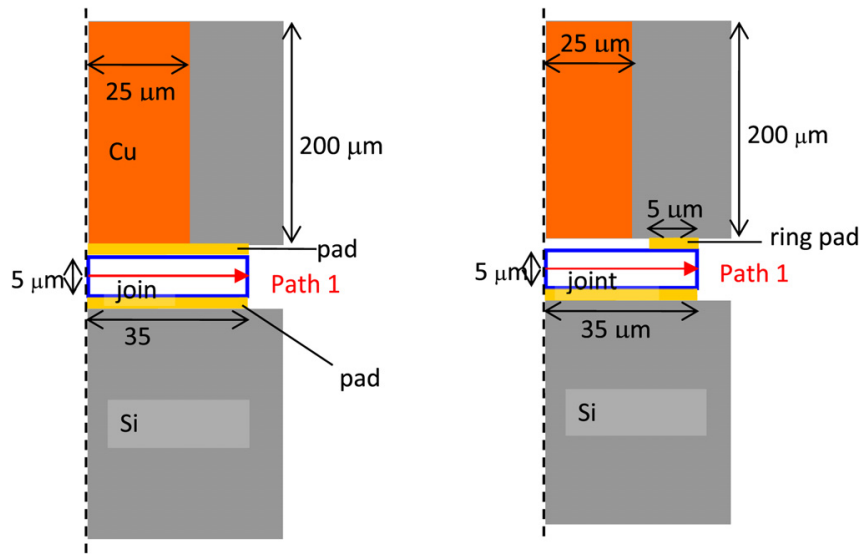

FIG. 89. Schematic diagram showing dimensions used in mechanical modeling for (a) the full pad and (b) the proposes pad (i.e., the ring pad). ${ }^{132}$ Reproduced with permission from Zhang et al., "A low stress bond pad design for low temperature solder interconnections on through silicon vias (TSVs)," IEEE Trans. Compon., Packag., Manuf. Technol. 1(4), 510 (2011). Copyright 2011 Institute of Electrical and Electronics Engineers.

a ring-shaped pad. In this structure, the joint wets only to the pad and not the traces or via in between. Such a design will effectively decouple the via from the micro bump, such that the micro bump is unaffected by any contraction of the TSV. This proposed design does not involve any additional fabrication or material cost. Fabrication of the pads only requires a change in the mask design to implement. Figure 90 shows that with the proposed pad design, the maximum tensile stress in the micro bump decreases by $50 \%$. Reliability assessment has also been done in order to compare the proposed pad design with the conventional design. It is found that the samples with the proposed design have a better drop impact reliability performance than the samples with the conventional full pad design. In this paper, a double-sided multilayer metallization process on $200 \mu \mathrm{m}$ TSV wafer with the low temperature and low volume lead-free solder ( $\mathrm{Sn}$ $(0.5 \mu \mathrm{m}) / \mathrm{In}(2 \mu \mathrm{m}) / \mathrm{Au}(0.05 \mu \mathrm{m}))$ has been demonstrated. Low temperature micro bump process for $\mathrm{C} 2 \mathrm{C}$ has also been established, which is highly desirable for next generation IC

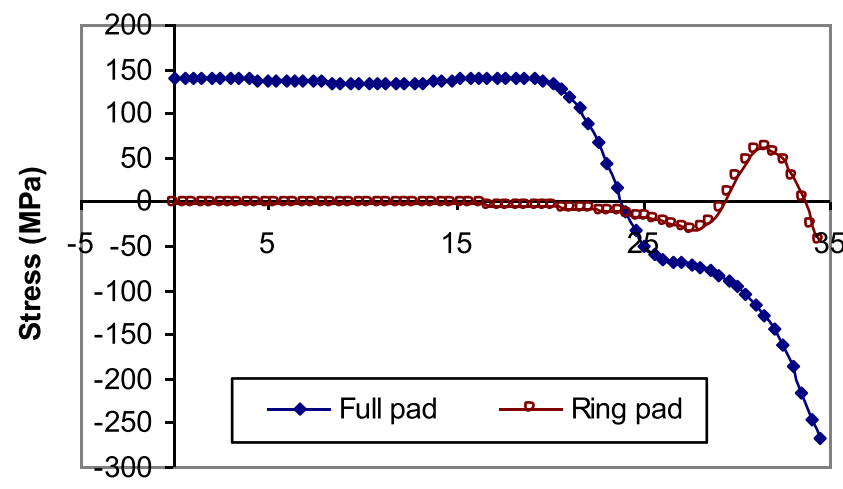

Distance along Path $1(\mu \mathrm{m})$

FIG. 90. Graph of stress (S33) along mid plane of bond for the full pad and the ring pad. ${ }^{132}$ Reproduced with permission from Zhang et al., "A low stress bond pad design for low temperature solder interconnections on through silicon vias (TSVs)," IEEE Trans. Compon., Packag., Manuf. Technol. 1(4), 510 (2011). Copyright 2011 Institute of Electrical and Electronics Engineers. packaging, where multifunctional chips such as memory, logic, and MEMS devices are used.

\section{EDA CONSIDERATIONS AND READINESS}

As with conventional ICs, readiness of the EDA tools is essential to sign-off TSI based 2.5D ICs. While choosing EDA platforms for 2.5D IC design, designers typically face a choice between using dedicated 3D EDA tools (i.e., 3D IC compiler $^{134}$ and 3D system-on-chip (SoC) Encounter ${ }^{135}$ ) and making necessary enhancements to existing industrystandard and proven 2D EDA platforms. In our work, we have relied on the latter since $2.5 \mathrm{D}$ ICs are a mid-point between traditional 2D ICs and 3D stacks. Moreover, we have developed a methodology to enhance existing 2D IC design tools to achieve 2.5D IC design closure on TSI which contains TSVs in a floating TSI substrate. In this section, we describe the methodology to incorporate various components of TSI technology (i.e., uBumps, BEOL/RDL lines, TSV, C4 bumps, etc.) in 2.5D TSI PDK using (1) technology layoutentry-format (LEF) files, (2) Parasitic Extraction (PEX) rule decks and TSV extraction, and (3) design Rule Checks (DRC)/Layout Versus Schematic (LVS) rule decks. TSV design rules along with TSV and C4 bump parasitic extraction form major modifications to enable 2D EDA tools to perform 2.5D IC design closure. PDK provides an infrastructure to support a reference EDA flow that enables the designer to sign-off 2.5D ICs. 2.5D TSI design flow composing of: (A) design import, (B) floor planning and placement, (C) routing on 2.5D TSI, (D) SI and PI verification, followed by (E) DRC and LVS verification is described. A sample case-study consisting of FPGA and memory foot print on 2.5D TSI is illustrated as well.

\section{A. 2.5D IC PDK}

2.5D PDK predominantly consists of (1) technology LEF files, (2) PEX rule decks and TSV extraction, and (3) DRC/LVS rule decks. These will be elaborated below.

\section{Technology LEF files}

The technology LEF files detail the routing and via layers description. Concurrently, viewer files are built to aid the viewing of the layers for some tools. The layer map is then used in the EDA flow to construct test chips as well as physical designs of 2.5D TSI. In addition to the technology LEF, block level LEF files describing physical co-ordinates of the I/Os corresponding to every block are also required during the physical design flow.

\section{PEX rule decks and TSV extraction}

The major difference between 2.5D TSI technology and traditional 2D IC technology is that the Si substrate is not grounded in 2.5D IC technology but is floating. The floating Si substrate acts as pseudo metallic plate for a typical Si substrate resistivity of $1-100 \Omega-\mathrm{cm}$. TSV is also different compared to the traditional BEOL wires as it is surrounded by $\mathrm{Si}$ substrate rather than the usual $\mathrm{SiO}_{2}$ or low-k dielectrics. The lumped "RC" model ${ }^{136}$ of the TSV is used to extract TSV as 


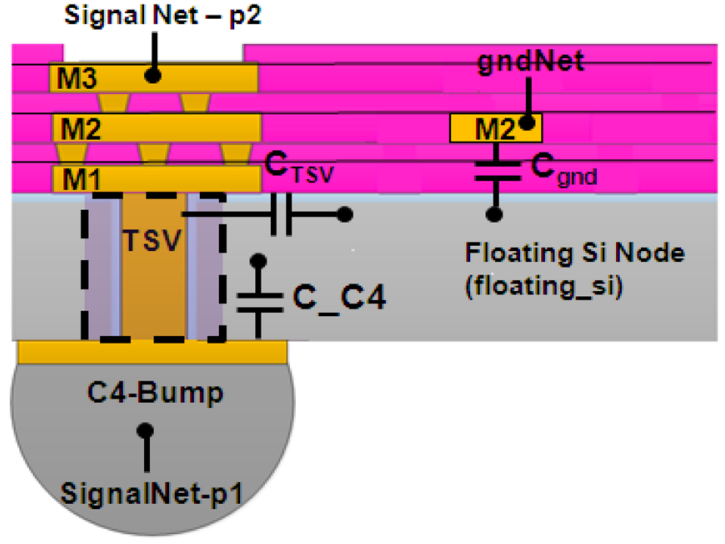

FIG. 91. Parasitic capacitances associated with TSV, C4 bump and ground net in $2.5 \mathrm{D}$ TSI.

a device. The $\mathrm{R}_{\mathrm{TSV}}$ and $\mathrm{C}_{\mathrm{TSV}}$ are obtained from the TSV daisy chain as well as the TSV embedded and de-embedded bank characterization structures detailed in Sec. IV. Temperature dependent TSV "RC" models derived through modeling ${ }^{136}$ or measurements can also be employed. Parallel plate model or characterized capacitance values for $\mathrm{C}$ _C4 are employed for $\mathrm{C} 4$ bumps. Parasitic capacitances associated with TSV, C4 bump, and ground net in 2.5D TSI are illustrated in Figure 91.

The process of building the PEX rule deck for front side BEOL or RDL interconnects is similar to traditional 2D interconnects and the characterized $\mathrm{RC}$ values of different routing layers and the via layers are incorporated to achieve parasitic extraction. However, the extraction methodology for TSV and $\mathrm{C} 4$ bump is different and follows a two-step extraction process that is described in this paper. The two step extraction methodology consists of (a) extracting TSV as well as the C4 bumps with respect to the floating Si substrate and (b) merging the netlist with other front side extracted nets with floating $\mathrm{Si}$ as a common node is employed. In an actual extraction netlist TSV and C4 bumps are modeled as lumped RC elements. Front side interconnects are extracted and merged with TSV and C4 bump extraction using the floating Si node. Consider a signal net starting from the $\mathrm{C} 4$ bump point Signal Net $-\mathrm{p} 1$ and ending on M3 at Signal Net-p2. The ground net is located at M2 with gndNet as a terminal. The resulting parasitic extraction model of the Signal Net is illustrated in Figure 92. The extracted TSV capacitance $\left(\mathrm{C}_{\mathrm{TSV}}\right)$ and $\mathrm{C} 4$ bump capacitance $\left(\mathrm{C}_{-} \mathrm{C} 4\right)$ and $\mathrm{C}_{\mathrm{gnd}}$ are merged on the floating Si node using the 2 step extraction methodology. The front side interconnects are extracted using the usual 2D extraction methodology. The associated paths on M1, M2, are extracted as R_M1, C_M1 and R_M2, C_M2, respectively, while vias $\mathrm{V} 1$ and $\mathrm{V} 2$ are extracted as resistances alone, R_V1 and R_V2, respectively.

\section{DRC/LVS rule decks}

\section{- DRC rule decks}

Similar to 2D ICs, 2.5D IC DRC are important to verify the manufacturability of the 2.5D IC. Similar to 2D ICs, minimum size and spacing constraints on the routing and via layers are captured as design rules in the 2.5D IC design rule decks. The design rules for the traditional BEOL and front side RDL technology are implemented in addition to the design rules for the $\operatorname{TSV}\left(\varphi_{\mathrm{TSV}}=10 \mu \mathrm{m}\right.$; Pitch $\left.=50 \mu \mathrm{m}\right)$ as shown in Figures 93 and 94, respectively. In addition, due to resulting dishing during via manufacturing for typical via size $>5-7 \mu \mathrm{m}$, stacked vias are difficult to manufacture and staggered vias are recommended as shown in Figure 95. With reduced via dimensions $<2 \mu \mathrm{m}$, it could be likely to achieve stacked vias if dishing can be controlled.

\section{- LVS rule decks}

As in 2D ICs, the LVS rules decks in 2.5D are critical to capture the errors in the $2.5 \mathrm{D}$ layout such that $2.5 \mathrm{D}$ physical design is in sync with $2.5 \mathrm{D}$ schematic/netlist. The connectivity information is obtained from schematic/netlist while the physical connections form the layout. LVS rules are essential to identify open/short errors in the layout.

\section{B. 2.5D IC EDA flow}

2.5D TSI design flow built leveraging existing 2D design tools is illustrated in Figure 96. The flow leverages the PDK elements along with the design inputs such as design netlist and the design constraints to accomplish the 2.5D IC design closure. The description of each major step is as follows.

\section{Design import}

A synthesized netlist is obtained from the front end design sign-off and is used to import the design. As the 2D IC netlist consists of gate-to-gate connections predominantly, 2.5D IC netlist describes the chip-to-chip connections

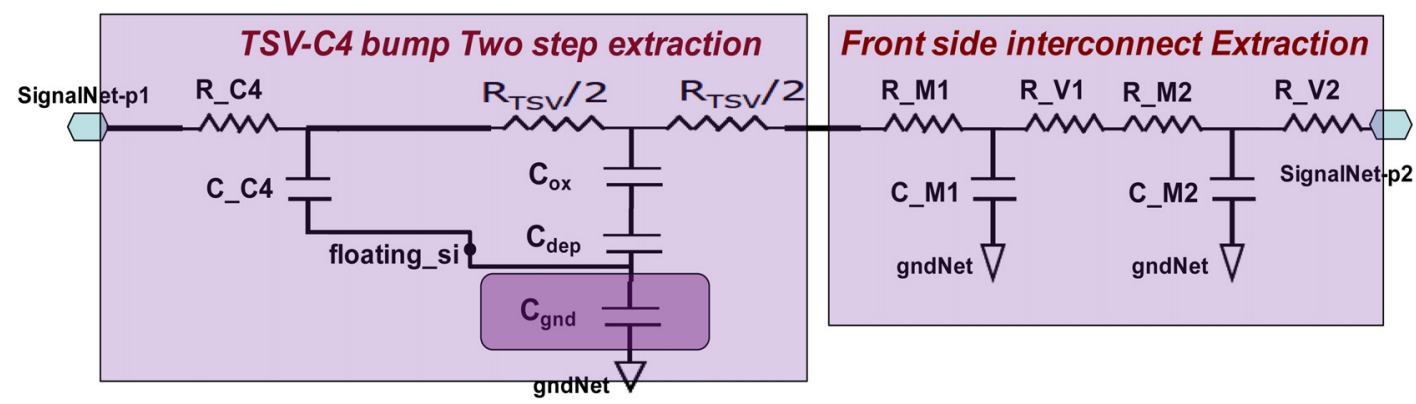

FIG. 92. Line extraction model in 2.5D TSI extracted using 2 step extraction methodology. 


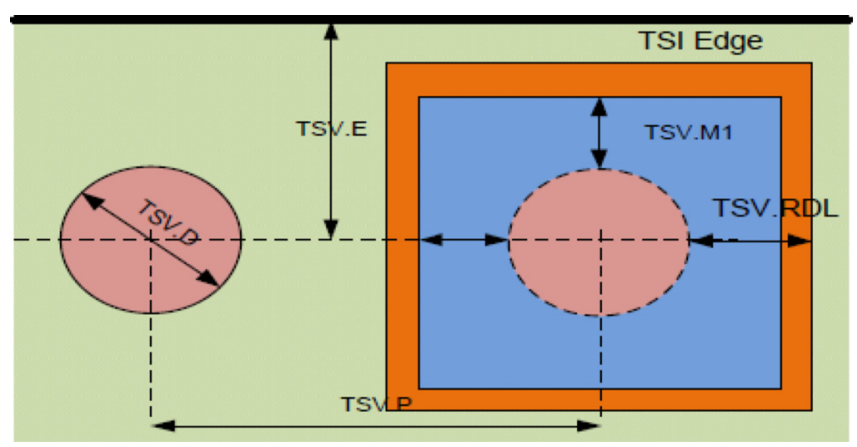

FIG. 93. Schematic illustrating the design rules for TSV.

as well as the chip-to-package I/O connections. Once the design is imported, the floor planning and placement of the chips on 2.5D TSI is performed as described in Sec. VII B 2.

\section{Floor planning and placement}

Floor-planning and placement of the guest dies are performed before routing the netlist. Floor planning, as well as Placement and Routing (PnR) require technology LEF and individual Library Exchange Format (LEF)/Design Exchange Format (DEF) description of the design blocks (i.e., FPGA and Memory blocks) for confirming the relative front-side micro-bump locations of individual blocks. Once the design is floor planned, the I/O terminals of the design are assigned to the back-side $\mathrm{C} 4$ bumps spaced regularly such that the routing distance between the back side $\mathrm{C} 4$ bumps and the front side micro-bumps is minimized. Once the location of the I/O terminals on the back side of the $2.5 \mathrm{D}$ TSI as well as the front-side micro-bump locations of the individual blocks is confirmed, the design is ready for routing.

\section{Routing on 2.5D TSI}

The connectivity of connections is specified in the synthesized netlist provided while the micro-bump locations specify the $x-y$ co-ordinates of the end points to be routed. The designers have an option to impose area constraints on the design in case certain area needs to be dedicated for power routing, etc. The infrastructure enables the designers to layout $2.5 \mathrm{D}$ circuits as seamlessly as they are used in layout 2D circuits. Moreover, TSVs are routed automatically and the environment can be leveraged to explore the impact of disparate wiring sizes and pitches on the design routing ability.

\section{Parasitic extraction}

Parasitic extraction is another critical step before SI and PI verification are performed. Two-step extraction methodology detailed in Sec. VII B is used for accurate TSV and C4 bump extraction. The post-layout extracted netlist is further used for Signal Integrity (SI) and Power Integrity (PI) verification.

\section{SI and PI verification}

To verify the SI of the design, the shortest, most typical, and longest lines in the design are extracted and simulated using spectre ${ }^{137}$ to perform eye-diagram simulations. System design constraints are considered for simulations as well. Static IR drop analysis is performed to verify the low resistance Power Delivery Network (PDN) for a specified current carrying the capacity of the chip.

\section{DRC and LVS verification}

After SI/PI verification and DRC/LVS confirmations, the sign-off.gds is generated for manufacturing purpose.

\section{2.5D functional vehicle design-A case study}

FPGA and DRAM integration ${ }^{138}$ is now shown to demonstrate the EDA flow that uses the 2.5D TSI PDK. Figure 97 shows the schematic of the design. Figure 98 depicts the results achieved at various steps in the EDA flow. Design import in step (a) is achieved prior to the FPGA and DRAM memory placement performed in step (b). The back side $\mathrm{C} 4$ bumps minimizing the routing distance is planned as well. Once the connectivity is known through the netlist and $\mathrm{x}-\mathrm{y}$ coordinates are determined after the placement of the FPGA and DRAM memory chips together with the back side C4 bumps, design is ready for routing. The resultant auto-routing with RDL technology (thickness $=3 \mu \mathrm{m}$, width $=5 \mu \mathrm{m}$, and pitch $=10 \mu \mathrm{m}$ ) achieved is depicted in step (c).

The schematic used to simulate the SI for timing verification is shown in Figure 99. $\mathrm{Z}_{\text {driver }}$ denotes the driver impedance while $\mathrm{C}_{\mathrm{tx}}$ and $\mathrm{C}_{\mathrm{rx}}$ denote the capacitance at the transmitter and receiver ends, respectively. The post layout extracted spice netlist of chip-2-chip interconnect line between the FPGA and DRAM memory blocks is used for spice simulations. Length of the extracted 2.5D TSI interconnections is $\sim 25 \mathrm{~mm}$. Eye diagram spectre $^{137}$ simulation results for different data rates, $\mathrm{Z}_{\text {driver }}$ and $\mathrm{I} / \mathrm{O}$ capacitances are shown in Figure 100 while Table $\mathrm{X}$ lists the eye height, eye width, and jitter estimated for these data rates. It could be readily seen that the eye width, eye height, as well as the jitter for the chip-2-chip interconnect line deteriorate with

\begin{tabular}{|l|l|l|l|}
\hline \multicolumn{1}{|c|}{ Symbol } & \multicolumn{1}{c|}{ Description } & \multicolumn{1}{c|}{ Min } & \multicolumn{1}{c|}{ Max } \\
\hline TSV.D & TSV diameter & $10 \mu \mathrm{m}$ & $10 \mu \mathrm{m}$ \\
\hline TSV.P & TSV pitch & $50 \mu \mathrm{m}$ & N.A. \\
\hline TSV.E & $\begin{array}{l}\text { TSV center to TSI edge } \\
\text { distance }\end{array}$ & $300 \mu \mathrm{m}$ & N.A. \\
\hline TSV.M1 & M1 overlap of TSV pad & $2 \mu \mathrm{m}$ & $12 \mu \mathrm{m}$ \\
\hline TSV.RDL & RDL overlap of TSV & $4 \mu \mathrm{m}$ & $\begin{array}{l}200 \mu \mathrm{m} \text { (When with the } \\
\text { C4 bump) }\end{array}$ \\
\hline TSV.d & TSV density & N.A. & $1 \%$ \\
\hline
\end{tabular}




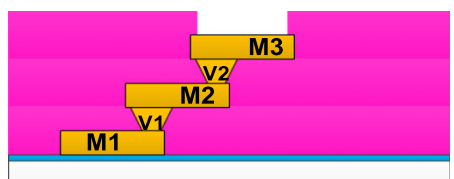

Displaced Vias $\rightarrow$ Mandatory

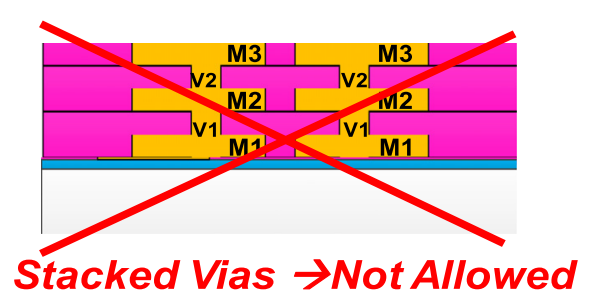

FIG. 95. Stacked vias are not allowed for front side RDL technology. increasing data rates. Moreover, driver impedance as well as I/O capacitances tuning are required to reach the desired speed of $2 \mathrm{GT} / \mathrm{s}$. The data-rate could further be improved by optimizing interconnect technology parameters such as width and spacing.

Constructing a robust PDN needs a strategic approach as it should be ensured that the resistance of the PDN is as minimal as possible. Dedicated metal layers mimicking the power and ground planes are constructed. The ground C4 bumps are connected to the ground plane directly using TSVs while the ground plane is slotted to realize the connection between the $\mathrm{C} 4$ bumps and the ground plane through parallel TSVs, as well as BEOL vias if required. Similarly, the power/ground plane is connected to the front side microbump using multiple vias and minimal routing on the metal layers. After PDN construction, post layout extraction of the PDN is performed and is simulated using the schematic view illustrated in Figure 101. Simulation results are used to validate the static IR drop on Voltage Drain Drain (VDD) as well as Voltage Source Source (VSS) rails. Current source mimicking the chip current consumption is a key input parameter to evaluate the IR Drop_VDD and IR_Drop_VSS obtained at the micro-bump of the FPGA and Memory guest dies.

For the given test case, current carrying capacity of the chip is considered to be $3 \mathrm{~A}$ with $\mathrm{V}_{\mathrm{dd}}=1 \mathrm{~V}$ and $\mathrm{V}_{\mathrm{ss}}=0 \mathrm{~V}$ biasing the back side $\mathrm{C} 4$ bumps. Test bench (.dc) simulation results at the front-side micro-bump locations estimate the IR drop of the $\mathrm{V}_{\mathrm{DD}}$ net to be $3.97 \mathrm{~V}$, while the IR drop at VSS net is estimated to be $1.02 \mathrm{mV}$. Thus, minimal IR drop ( $\sim 10 \% \mathrm{Vdd})$ suggests that PDN performance is acceptable, provided that the IR drop falls within desired limits. Such low value of the IR drop is due to highly conductive PDN constructed through slotted cheese architecture and low impedance vias.

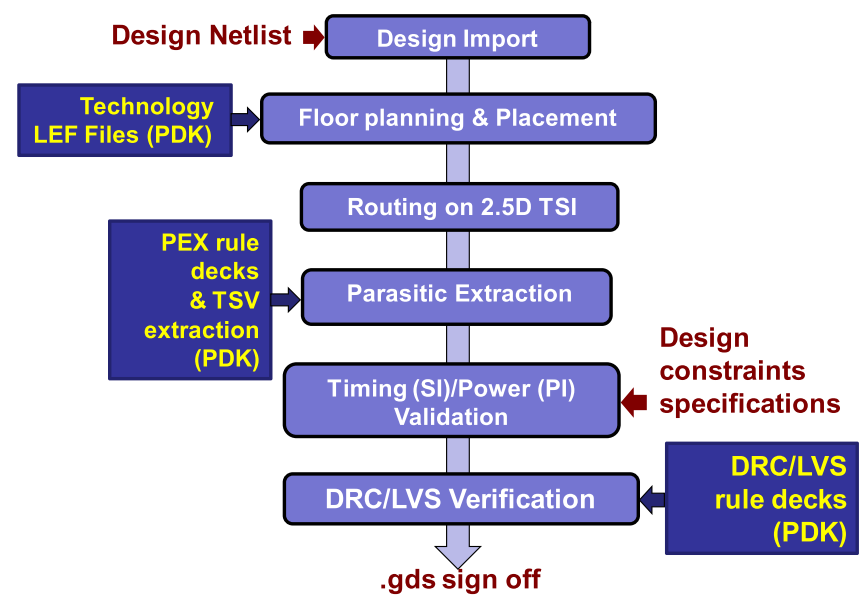

FIG. 96. 2.5D IC physical design flow.

\section{REVISITING APPLICATIONS AND BENEFITS OF 2.5D TSI TECHNOLOGY}

Application requirements and technology development are significantly interlaced. While advanced state-of-the-art application requirements drive technology nodes for numerous advanced technologies, advanced technologies in many domains do act as a catalyst realizing niche applications. In this section, the application roadmap that could potentially leverage 2.5D TSI technology is addressed followed by $2.5 \mathrm{D}$ technology component specifications and integration schemes to enable novel applications.

\section{A. 2.5D TSI technology-Application space}

The proliferation of electronic devices has been unprecedented in a modern day human life. The growing demands of connectivity/internet along with smart applications in various industrial/business sectors, such as media, gaming, automobile, industry, bio, defense, and space, have lead to a mammoth growth of data generation and increased the storage and processing requirements. As a cliché, mankind is generating more data in two days than we did from the dawn of man until 2003. ${ }^{139}$ It is forecasted that the diverse and exploding digital universe is experiencing the information doubling every 18 months. ${ }^{140}$ Today, existing number of digital bits is more than stars in the universe and the data is growing at a phenomenal factor of 10 every 5 years. Big data storage, processing/analysis, etc., can only be supported through continuous technology innovations and semiconductor industry supporting the foundation of the pyramid of electronic innovation needs to enable efficient (i.e., low power and low area/form factor) and high data rate data centers, communication fabrics, mobile, as well as sensing device networks.

At subsystem level, the demand for moving large volumes of data translates into the ability to exchange information between logic ICs and Memory ICs. 2.5D TSI technology provides a new way to interconnect ICs in a manner that provides higher performance at lower power consumption. Over the next few years, this technology will enable design and manufacture of (a) Graphics Processing Units, smartphones, tablets which need longer battery life, (b) data-centers that handle larger volumes of information, yet consuming

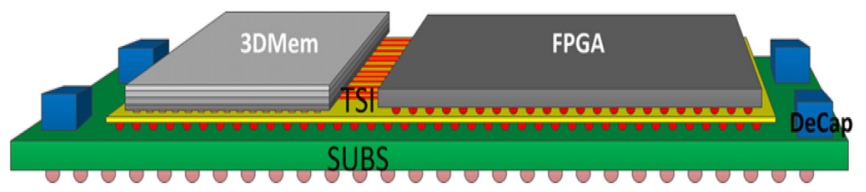

FIG. 97. Schematic of the FPGA and DRAM memory integration on 2.5D TSI. 


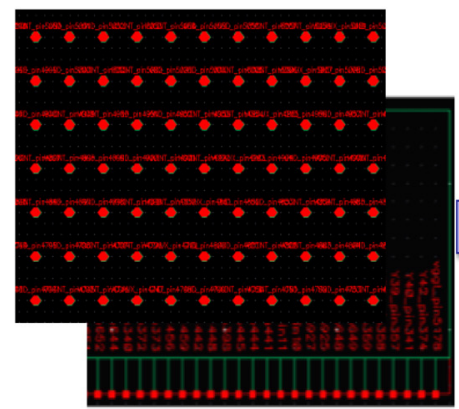

(a) Design Import

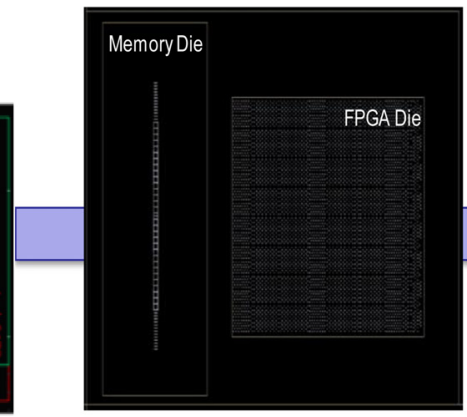

(b) FPGA \& Memory placement

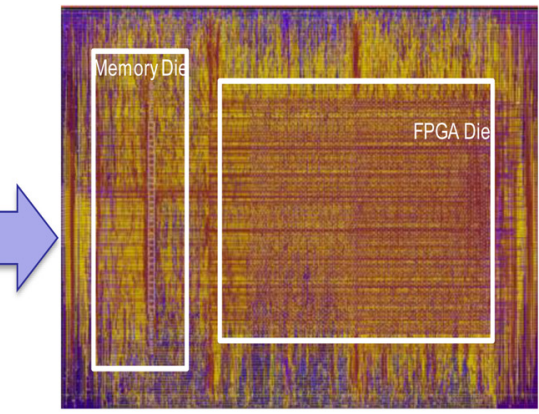

(c) Auto-routed 2.5D TSI design with TSVs

FIG. 98. From Netlist entry to auto routed design with TSVs. (a) Design import, (b) FPGA and memory placement, and (c) auto-routed 2.5D TSI design with TSVs.

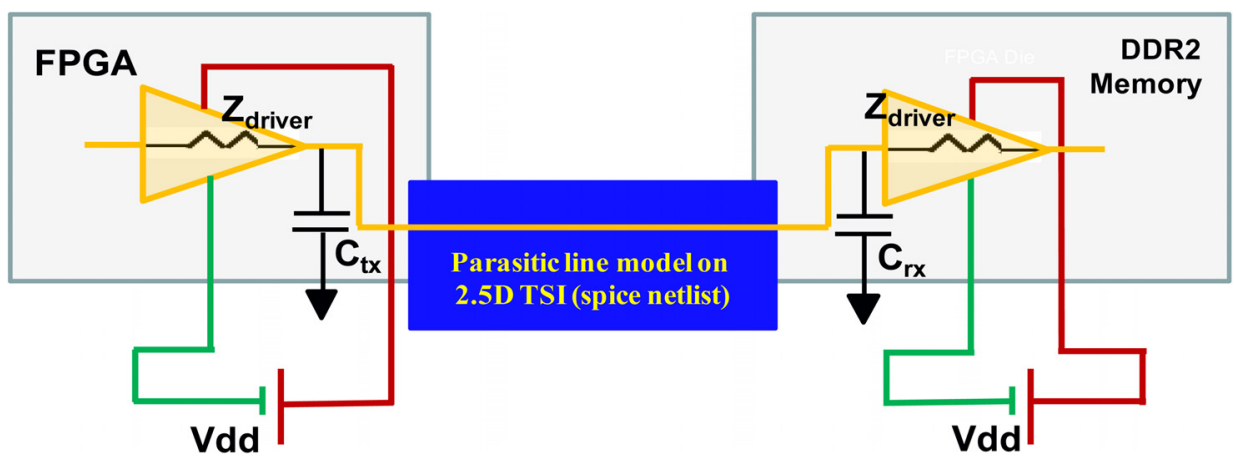

FIG. 99. Schematic to simulate the SI of a line in $2.5 \mathrm{D}$ TSI.

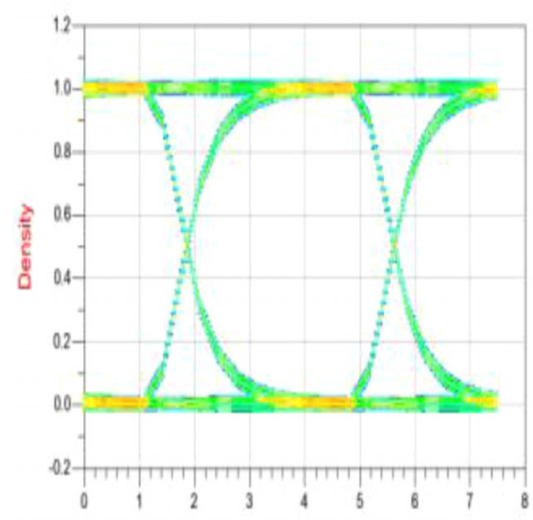

(a) $\mathrm{C}_{\mathrm{tx}}=0.5$

Data rate $=0.26$

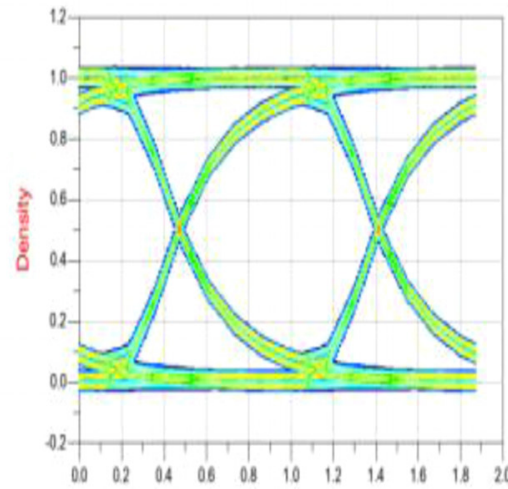

(b) $\mathrm{C}_{\mathrm{tx}}=0.5$ Data rate $=1.06$

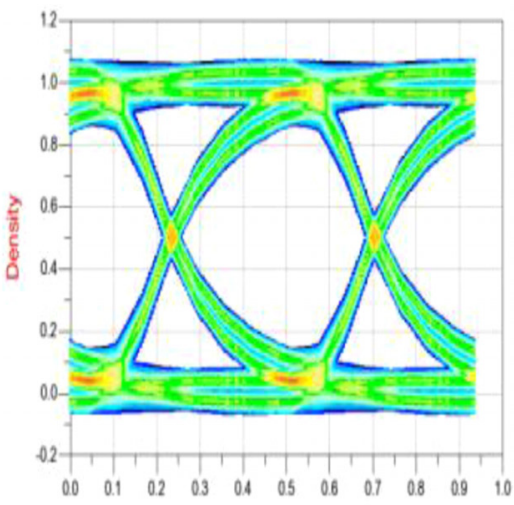

(c) $\mathrm{C}_{\mathrm{tx}}=0.5$

Data rate $=2.1$

FIG. 100. Eye diagram simulations for various data rates and driver impedances $(0.26 \mathrm{GT} / \mathrm{s}-2.1 \mathrm{GT} / \mathrm{s})$. (a) $\mathrm{C}_{\mathrm{tx}}=0.5$ data rate $=0.26$, (b) $\mathrm{C}_{\mathrm{tx}}=0.5$ data rate $=$ 1.06 , and (c) $\mathrm{C}_{\mathrm{tx}}=0.5$ data rate $=2.1$.

TABLE X. Eye diagram simulation results analysis for FPGA-DRAM interconnect lines at various data rates/frequencies.

\begin{tabular}{|c|c|c|c|c|}
\hline $\mathrm{Z}_{\text {driver }}$; bit rate $@ \mathrm{~V}_{\mathrm{dd}}=1 \mathrm{~V}$ & $\mathrm{C}_{\mathrm{tx}} / \mathrm{C}_{\mathrm{rx}}(\mathrm{pF})$ & Eye height $\left(\% \mathrm{~V}_{\mathrm{dd}}\right)$ & Eye width $(\%)$ & Jitter (\%) \\
\hline (a) $50 \Omega ; @ 0.26 \mathrm{GT} / \mathrm{s}$ & $0.5 / 2$ & 91 & 99 & 1 \\
\hline (b) $20 \Omega ; @ 1.06 \mathrm{GT} / \mathrm{s}$ & $0.5 / 2$ & 68 & 96 & 4 \\
\hline (c) $10 \Omega ; @ 2.12 \mathrm{GT} / \mathrm{s}$ & $0.5 / 0.5$ & 59 & 91 & 9 \\
\hline
\end{tabular}




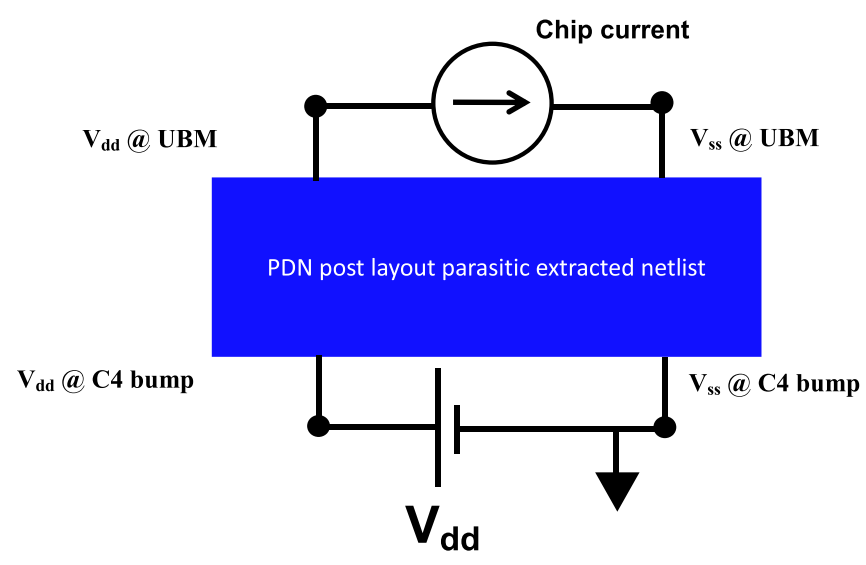

FIG. 101. Schematic for static IR Drop analysis.

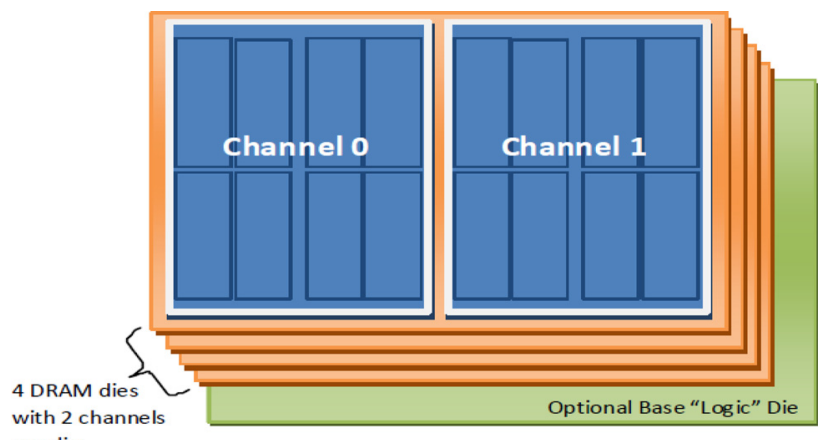

per die

FIG. 102. Schematic diagram of an 8-channel high bandwidth memory. ${ }^{14}$

lower power compared to its earlier generations, and (c) high performance supercomputers. As with a technology, that is in its early adoption phase, 2.5D TSI has challenges which the industry and R\&D community needs to address. These challenges stem mainly from the cost of transitioning from a well established semiconductor IC manufacturing flow to allow for designing, fabricating, and assembling 2.5D ICs on the TSI platform. With rising amounts of internet data traffic that needs to be handled, over the next decade, 2.5D TSI technology will gradually gain market adoption.

\section{B. Application roadmap}

Over the next 5 years, state of the art GPU, FPGA, and data centers applications will drive the density of electrical interconnects on TSI. In 5-10 years, high-end data centers and high performance computing applications will drive the adoption of optical interconnects on 2.5D TSI platform. This was depicted in Figures 6 and 7. Availability of $\mathrm{HBM}^{14}$ (see Fig. 102) DRAM devices will drive the adoption of high density electrical interconnects on TSI. Such high density electrical interconnects will drive the use of multiple HBM devices that can be stacked around a GPU device ${ }^{141}$ as shown in Figure 103. The figure indicates the increasing processing capability that can be supported by a GPU that uses 2.5D TSI technology.

With increasing volume of data traffic in servers and data centers, optical interconnects become a critical element in a Data center. A typical data center consists of servers that receive data, switches that route the data, and storage systems that keep data in storage (for example, emails, video, photographs, etc.). In such cases, multiple channels each with data-rates of $100 \mathrm{Gbps}$ will be needed between racks across the data center (over interconnects that are between $10 \mathrm{~m}$ and $300 \mathrm{~m}$ ). Continuing to use $\mathrm{Cu}$ interconnects will drive up power consumption in data centers. In such cases, optical fibers will need to pipe data to and from logic ICs. Around 5-10 years out, this will drive extensive adoption of electronic photonic integrated circuits which bring the ability to convert high-speed SERDES electrical signals into optical signals inside the rack-unit lowering power consumption significantly.

\section{FUTURE OUTLOOK/CONCLUSIONS}

\section{A. 2.5D TSI technology roadmap}

The next generation VLSI applications project the number of transistors double every 18 months (Ref. 2) and I/Os also increase in tandem as per Rent's rule. ${ }^{4}$ As these next generation chips are mounted on the interposer, the wiring density and infrastructure demands increase as well. The key

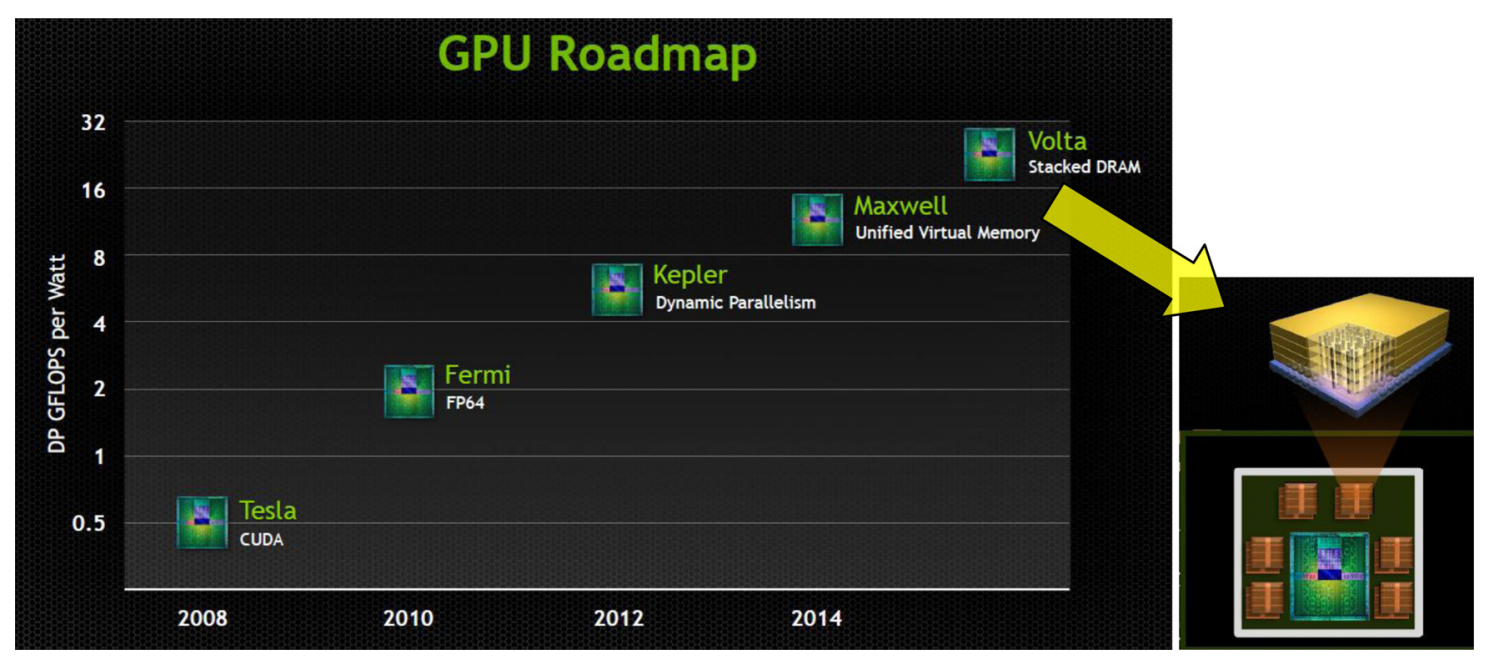

FIG. 103. Graphics processing unit (GPU) roadmap from Nvidia. ${ }^{141}$ Reproduced with permission from J.-H. Huang, "Connecting people with ideas," paper presented at GPU Technology Conference, Nvidia, 2013. Copyright 2013 Nvidia Corporation. 


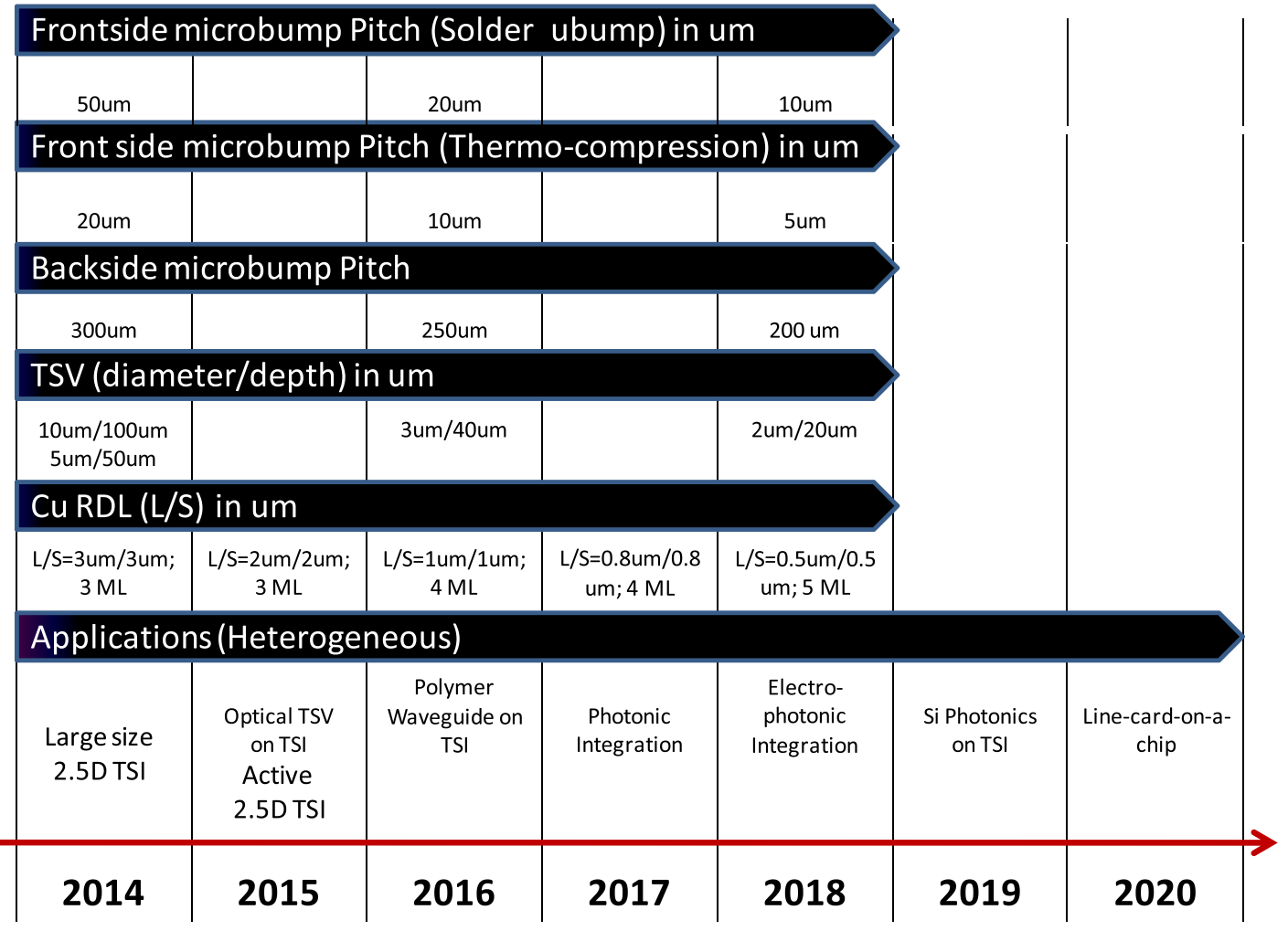

FIG. 104. Roadmap for different components of 2.5D TSI technology.

technology components, such as micro-bumps, $\mathrm{Cu}-\mathrm{RDL}$ and dual damascene BEOL line width and pitches, via and enclosure sizes, TSV, back side micro-bump, and size of the interposer, need to be scaled concurrently. In sync with the ITRS roadmap, ${ }^{3}$ the proposed TSI component technology road map detailing the time lines for component scaling is illustrated in Figure 104.

Contemporary TSVs with $10 \mu \mathrm{m}$ diameter and $100 \mu \mathrm{m}$ height in $2.5 \mathrm{D}$ TSI are projected to be scaled to $2 \mu \mathrm{m}$ diameter and $20 \mu \mathrm{m}$ height in the future. The technology scaling imposes challenges to etch and fill small diameter high aspect ratio TSVs as well as to handle the thin wafer processing. ${ }^{142,143}$ While the sub-micrometer Cu-damascene BEOL interconnects already exist, the emerging polymer based $\mathrm{Cu}-\mathrm{RDL}^{9}$ approach provides an alternate low cost outsourced semiconductor assembly and test (OSAT) based manufacturing approach. The existing dimensions of $3 \mu \mathrm{m}$ width and $6 \mu \mathrm{m}$ pitch with 3 metal layers stack are projected to be scaled to $0.5 \mu \mathrm{m}$ width, $1 \mu \mathrm{m}$ pitch, and $4-5$ metal layers stacks on interposers by 2018. As via could form a bottle neck for potential routing, existing $7 \mu \mathrm{m}$ via sizes and $12 \times 12 \mu \mathrm{m}$ enclosures in $\mathrm{Cu}-\mathrm{RDL}$ technology is recommended to be scaled to $3 \mu \mathrm{m}$ via size and $5 \times 5 \mu \mathrm{m}$ enclosure or finer. Front side micro-bumps are usually staggered with $25 \mu \mathrm{m}$ size and $50 \mu \mathrm{m}$ pitch should progressively scale to $15 \mu \mathrm{m}$ size and $30 \mu \mathrm{m}$ pitch while the back side microbumps forming a $150 \mu \mathrm{m}$ diameter and $300 \mu \mathrm{m}$ pitch arranged in as a full array are likely to scale to $100 \mu \mathrm{m}$ diameter with $200 \mu \mathrm{m}$ pitch. The area of the interposers is expected to be as large as $40 \times 40 \mu \mathrm{m}$ with $50 \mu \mathrm{m}$ thickness Si wafer to accommodate large size $2.5 \mathrm{D}$ system designs.
The warpage as well as reliability challenges ${ }^{144}$ associated with building up the large size thin $2.5 \mathrm{D}$ TSI should be confronted as well.

Collectively, there are numerous challenges in manufacturing next generation 2.5D TSI. Few of them include correct bonding technology and materials, method of reducing TSV related stress, under-fill materials and method of application, as well as process reliability and yield. Increase of interconnect density requires a concomitant decrease of TSV diameter, increase of aspect ratio, and several layers of BEOL/ RDLs. Thinning Si wafer in order to reduce TSV aspect ratio has reached to its limits at around $20 \mu \mathrm{m}$ upon consideration of wafer or chip handling viewpoints. Also, the effect of mechanical stress and crystal damage is found to be enhanced when the $\mathrm{Si}$ thickness is reduced to below $20 \mu \mathrm{m} .{ }^{145}$ Possible causes include wafer thinning that introduces crystal damages, various films deposited on Si that introduces stress, and CTE mismatch between bonding agents and $\mathrm{Si}$ as the bonding is performed at higher temperatures. This imposes new requirements in finding improved process flows in making TSV, particularly with aspect ratio exceeding 10. Development of highly conformal etching and stress-free thin film deposition requirements in making high density TSV interconnects is mandated as well.

\section{Solder technology}

Conventionally, stacking one chip on the other is carried out using solder based bonding. With the trend of scaling down of interconnect pitches, the solder based bonding evolved from reflow BGA to thermo-compression $\mathrm{Cu}$ pillars with solder. Use of $\mathrm{Cu}$ pillar with solder with thermo- 


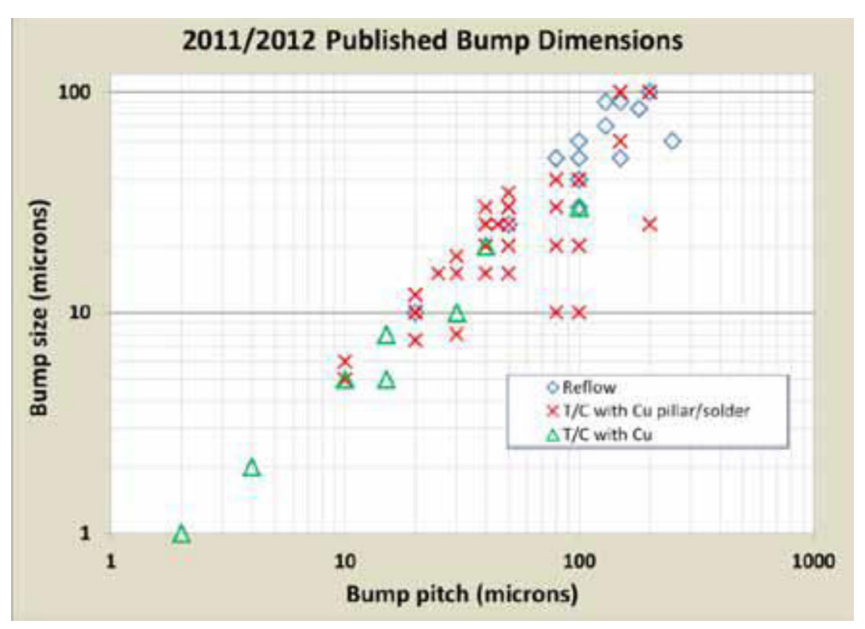

FIG. 105. Chip stacking bump sizes and pitches. ${ }^{146}$ Reproduced with permission from Bersch et al., "Cu-Cu direct bonding for ultra-high density chip-to-chip interconnects," in Chip Scale Review Magazine, Jul/Aug 2013, p. 20. Copyright 2013 Chip Scale Review.

compression bonding is found to have a limit with the scaling down of interconnect pitch below $20 \mu \mathrm{m}$. Melting solder under force tends to squeeze out and bridge with neighboring interconnects, causing reliability issues. This shows the limit of solder-assisted chip stacking and paves the way for metalmetal diffusion bonding. The trend of chip stacking methods is summarized in Figure 105. ${ }^{146}$

There are numerous publications targeting to extend the solder-assisted chip stacking such as the development of bump-less technology and low-temperature eutectic bonding. ${ }^{147-149}$ The work carried out by Lin et al., wherein solder paste reflow or electroplated $\mathrm{Cu}$ is applied with fan-out configuration for Comic-Con International (CCI). However, this approach is more focused on low-cost manufacturing process than scaling down of the interconnect pitch. ${ }^{148}$

Generally, $\mathrm{Cu}$ pillars come with few microns solder layer for thermo compression bonding. The advantage of thick solder layer is that it absorbs any height variation of $\mathrm{Cu}$ pillars during thermo compression bonding. Minimizing solder thickness can prevent inter-bridging of interconnect. With the thinning of solder layer, the height uniformity of $\mathrm{Cu}$ pillar has to be improved as thin solder layer cannot absorb any height variation. Surface planarization before solder deposition can meet this requirement. From technology point of view, this method extends the applicability of $\mathrm{Cu}$ pillar for further scaling down to $10 \mu \mathrm{m}$ or below. However, from fabrication point of view, it adds additional processing steps leading to higher manufacturing cost.

The industry has already completed the transition from solder reflow to thermal compression bonding (TCB) with solder, which is usually referred as TC with $\mathrm{Cu}$ pillars. Transition from $\mathrm{Cu}$ pillar to direct $\mathrm{Cu}-\mathrm{Cu}$ bonding without solder is evident but there are many technical hurdles before realizing a commercially viable solution. This is expected to take place with $5 \mu \mathrm{m}$ $\mathrm{Cu}$ pillar at about $10 \mu \mathrm{m}$ pitch. The timeline and pitch limit for the actual transition in high volume manufacturing is hard to predict as it depends on many factors such as infrastructure, cost, reliability, and most importantly application drivers.

\section{Metal-metal bonding}

The transition from solder based thermos-compression bonding to solder-less metal-metal diffusion bonding is essential in realizing high density micro-bumps. To date, $\mathrm{Au}-\mathrm{Au}$ and $\mathrm{Cu}-\mathrm{Cu}$ are the most studied metals for chip stacking with metal diffusion bonding. ${ }^{150,151}$ Gold has no surface oxide thus eliminate surface cleaning issues before bonding. Conversely, $\mathrm{Cu}$ is more susceptible for surface oxidation and requires additional processing steps for surface cleaning.

There are many work reported on $\mathrm{Cu}-\mathrm{Cu}$ diffusion boding for $\mathrm{CCI}$ at various conditions. ${ }^{151-153} \mathrm{Cu}-\mathrm{Cu}$ bonding essentially comes with $\mathrm{Cu}$ pillars with diameter smaller than $10 \mu \mathrm{m}$ and aspect ratio below 1 . Cu can get deformed during bonding and absorbs minute changes in height variations. Nevertheless, as the overall height is below $10 \mu \mathrm{m}$, there is no room to absorb higher height variation particularly of that generated during $\mathrm{Cu}$ electroplating. This can result in cold or un-bonded joints. Thus, surface planarization before bonding becomes an essential step. There are two methods for surface planarization of $\mathrm{Cu}$ pillars, namely, CMP and bit grinding. CMP yields smoother surface with surface roughness below $1 \mathrm{~nm}$ although the operation cost is relatively higher. Bit grinding technology provides low cost approach for surface planarization but needs to trade off in terms of greater surface roughness. Under the optimized conditions, surface roughness can be as low as $15 \mathrm{~nm}$. This surface roughness gets easily deformed under the bonding force, thus it does not pose a challenge for $\mathrm{Cu}-\mathrm{Cu}$ bonding.

Normally, there is a thin layer of $\mathrm{Cu}$ oxide on the $\mathrm{Cu}$ surface. The brittle oxide layer breaks down during diffusion bonding under applied bonding force and creates diffusion centers wherein $\mathrm{Cu}$ atoms mutually diffuse into the other side of the $\mathrm{Cu}$ pillar. The oxide layer is a barrier for the diffusion and retards the diffusion rate. To overcome this drawback, $\mathrm{Cu}-\mathrm{Cu}$ bonding is usually carried out at higher temperature, usually over $350{ }^{\circ} \mathrm{C}$ providing adequate thermal energy for diffusing $\mathrm{Cu}$ atoms.

Removal of surface oxide before $\mathrm{Cu}-\mathrm{Cu}$ bonding can lower the bonding temperature even below $200{ }^{\circ} \mathrm{C}$. There are several methods in removing surface oxides such as (i) use of $\mathrm{H}_{2}$ at $350{ }^{\circ} \mathrm{C}$ or over, (ii) use of $\mathrm{H}_{2}$ plasma at low temperature $(<100 \mathrm{C})$, (iii) Ar sputter cleaning, (iv) use of formic acid gas, and (v) wet etching. Hydrogen plasma and the formic acid gas treatment are by far the most used techniques to date in removing $\mathrm{Cu}$ oxide.

Surface planarization before $\mathrm{Cu}-\mathrm{Cu}$ bonding is found to have two advantages. ${ }^{152}$ First, owing to the uniform $\mathrm{Cu}$ pillar height bonding, reliability is improved. Second, during the planarization process using bit grinding, the $\mathrm{Cu}$ surface approaches more amorphous-like structure. ${ }^{152}$ Thickness of amorphous-like layer is observed to be over $100 \mathrm{~nm}$. Electroplated $\mathrm{Cu}$ usually comprises $\mathrm{Cu}$ grains and this further recrystallizes after thermal annealing at or over $200{ }^{\circ} \mathrm{C}$, which is a normal practice to harden the $\mathrm{Cu}$ to prevent electro-migration. $\mathrm{Cu}$ diffusion during $\mathrm{Cu}-\mathrm{Cu}$ diffusion bonding occurs through grain bounders or through damaged grain surfaces. Use of electroplated and thermally annealed $\mathrm{Cu}$ yields smaller effective surface area for diffusion 
bonding. Generation of amorphous-like layer after bitgrinding is therefore a beneficial feature to increase the effective diffusion surface area. Moreover, this feature also assists in reducing the bonding temperature even below $200^{\circ} \mathrm{C}$. It has been observed that the amorphous-like $\mathrm{Cu}$ layer generated during surface planarization is only created by bit grinding process. The CMP process does not yield such an amorphous like structure.

As-deposited $\mathrm{Cu}$ begins to recrystallize at $200{ }^{\circ} \mathrm{C}$ or over. ${ }^{143}$ Some work has observed that the recrystallization process can initiate even at $180{ }^{\circ} \mathrm{C} .{ }^{142}$ Accordingly, the amorphous-like $\mathrm{Cu}$ layer can again recrystallize during surface de-oxidation step if the de-oxidation is performed at a temperature above $180^{\circ} \mathrm{C}$. Recrystallization negates the advantage of generating diffusion-friendly amorphous-like layer. The surface de-oxidation with $\mathrm{H}_{2}$ plasma is usually carried out at low temperature, for example, at room temperature. The wafer gets heated slightly only by the plasma, which does not exceed over $50^{\circ} \mathrm{C}$. Therefore, there will be no effect on amorphous-like layer with this de-oxidation method. The surface de-oxidation with formic acid gas begins at around $150{ }^{\circ} \mathrm{C}$ but is most effective at $200^{\circ} \mathrm{C}$. However, in order to prevent $\mathrm{Cu}$ recrystallization, formic acid treatment has to be carried out below $180^{\circ} \mathrm{C}$.

The decision to port an application to a particular technology depends on the routing ability requirements and SI/PI demands of the design. The designs with large number of I/ Os exhibiting larger signal and power routing requirements would prefer fine $\mathrm{Cu}$-Damascene BEOL routing to meet the routing requirements. Polymer based $\mathrm{Cu}-\mathrm{RDL}$ technology, offering coarse pitch although provides better SI performance and enhanced data rate per wire compared to $\mathrm{Cu}$ damascene technology, is better suited for designs where their routing requirements are satisfied.

As contemporary 2.5D TSI does not have any active elements on $\mathrm{Si}$, it is prudent and certainly economical to utilize the $\mathrm{Si}$ real estate for $2.5 \mathrm{D}$ system design. A potential $2.5 \mathrm{D}$ system schematic illustrating the memory and App processor integration on TSI is shown in Figure 106. Peripheral blocks, such as I/O, electrostatic discharge (ESD) protection, power management, RF and analog components, and IPD, can be housed in the $\mathrm{Si}$ substrate of active 2.5D TSI. ${ }^{154-158}$ Integrated Voltage Regulators (IVRs) can be used for improved power integrity while active transistors in TSI's $\mathrm{Si}$ substrate can be used for repeater insertion to build interblock optimized interconnects. TSVs built using Via-last technology are preferred for active interposers as the active devices can be manufactured in the foundry end and the wafers can be passed to OSATs or other lower cost packaging houses for TSV manufacturing and assembly. The impact of TSV-last ${ }^{146}$ technology on the transistor performance $^{147,148}$ should be evaluated as well.

Moreover, as 2.5D TSI technology would be catering to sensor as well as optical technologies co-existing with $2.5 \mathrm{D}$ electrical systems, the process integration schemes to accomplish such integration will be developed. Process flows integrating optical TSVs as well as polymer based waveguides to 2.5D TSV and $\mathrm{Cu}$-damascene and RDL integration flow will be developed. The infrastructure to analyze and design the opto-electrical as well as the electro-mechanical systems would be developed in parallel too.

While development and integration of new technologies on 2.5D TSI technology platform is an enabler to port new applications on 2.5D TSI, identifying the ownership of individual component makers is crucial for the success of steering 2.5D TSI technology to manufacturing mainstream. The testing and debugging methods to evaluate the components and process at every stage are likely to play a very important role. Ultimately, the $2.5 \mathrm{D}$ TSI is envisioned to be treated as a well-tested package before the expensive and off-the-shelf guest dies and components are mounted on it though an assembly and package flow.

\section{B. 2.5D/3D EDA outlook}

Although we have been able to design the $2.5 \mathrm{D}$ IC designs using the existing industry standard 2D IC design tools, it would be extremely useful to have dedicated 3D design tools for the technology path finding as well as design planning. There are industry standard 3D IC tools made available from Cadence ${ }^{135,159}$ and Synopsys. ${ }^{134}$ However, designers face the following shortcomings while employing them for 2.5D/3D IC sign-offs.

\section{Inability of existing 3D EDA tools to analyze unified $3 D$ netlist}

Analysis of the $2.5 \mathrm{D} / 3 \mathrm{D}$ power and clock distribution schemes is an important design component for 2.5D and 3D ICs. The 3D EDA tools unfortunately, do not allow the analysis of unified 3D netlist as it deals with the netlist on each stack of 3D IC individually. It would be beneficial to have a 3D design environment that is able to analyze unified 3D netlist. Moreover, it would enable automatic Clock Tree Synthesis (CTS), repeater insertion, etc., towards building a reliable 3D IC tape out. The infrastructure can then be leveraged to build the methodologies to formulate the $2.5 \mathrm{D} / 3 \mathrm{D}$ clock and power distribution networks benefitting the $2.5 \mathrm{D} / 3 \mathrm{D}$ system performance.

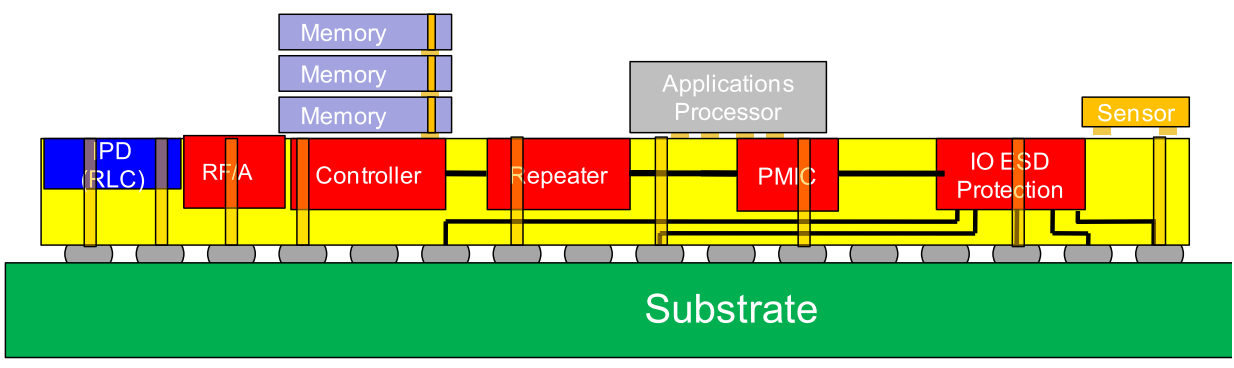

FIG. 106. Different design components on 2.5D active interposer-A schematic. 


\section{Employing calibrated TSV lumped " $R C$ " model}

The EDA tools support the BEOL via resistance to be specified in technology files detailing the interconnect layer descriptions. However, specifying via capacitance is not permitted in the technology description format. In case of TSV, as a part of 2.5D and 3D circuit, the TSV capacitance is the most dominant electrical parameter ${ }^{95}$ and hence it cannot be ignored. The present $2.5 \mathrm{D}$ and $3 \mathrm{D}$ tools do not allow an easier way to consider the TSV capacitance. This inhibits comprehensive $2.5 \mathrm{D} / 3 \mathrm{D}$ circuit and system analysis. The EDA tools enabling the incorporation of TSV/via capacitance in the technology description format would aid in the analysis of complex $2.5 \mathrm{D} / 3 \mathrm{D}$ systems and is desirable. An alternate way to consider TSV capacitance is to treat TSV as a threeterminal device instead of via. While TSV implemented as a device can be used to analyze small $2.5 \mathrm{D}$ and $3 \mathrm{D}$ circuit paths, the same methodology becomes slower for analyzing and back-annotating larger circuit blocks (>1000 gates).

\section{SUMMARY}

In this paper, we outline the advantages of implementing 2.5D ICs on TSI along with the key focus areas in implementing TSI technology. In addition, we highlight the solutions to surmountable challenges faced by this technology on its path to high volume adoption in mobile devices and servers/data centers. Logic and memory VLSI systems are the first and foremost applications that are ideally suited for steadfast 2.5D TSI implementations. Fabrication flow illustrating the TSV module development and its integration with the BEOL and/or RDL technology are presented in addition to various plausible assembly schemes and challenges. Electrical test characterization structures for technology characterization as well as PDK model development are described along with the EDA flow leveraging existing industry standard 2D IC tools to design, verify, and manufacture 2.5D ICs. Thermal modeling and simulations to alleviate the heat dissipation providing adequate cooling are analyzed for 2.5D TSI systems. FEM based thermo-mechanical analysis of the 2.5D TSI technology to address the manufacturability, warpage, and solder reliability challenges are performed as well. A suggested roadmap of individual 2.5D TSI components is presented so that the 2.5D TSI technology can potentially cater to numerous VLSI applications. For volume adoption of 2.5D TSI technology, the industry needs to focus on providing an end-to-end manufacturing platform for seamless integration of PDK, design, fabrication, test, and assembly of $2.5 \mathrm{D}$ system on TSI. This will enable high performance, low power designs at reduced design and manufacturing costs, as well as drive start-ups, fab-less design houses, integrated device manufacturers (IDM), OSATs, and foundries to design and manufacture 2.5D TSI products that meet/ exceed market expectations.

\section{ACKNOWLEDGMENTS}

The authors would like to gratefully acknowledge the support from IME's industry collaborators: Applied
Materials (AMAT), EVG, KMG chemicals, Qualcomm, STATS ChipPAC, Synopsys, Tezzaron Semiconductor, United Microelectronics Corporation (UMC), and United Test and Assembly Center (UTAC).

${ }^{1}$ R. Wilson, Big Data: Where It Comes From, Where It Will Go, see http://www.altera.com/technology/system-design/articles/2014/big-dataevolution.html

${ }^{2}$ G. E. Moore, "Cramming more components onto integrated circuits," Proc. IEEE 86(1), 82 (1998).

${ }^{3}$ Semiconductor Industry Association, International Technology Roadmap for Semiconductors, 2010.

${ }^{4}$ B. S. Landman and R. L. Russo, "On a pin versus block relationship for partitions of logic graphs," IEEE Trans. Comput. C-20(12), 1469 (1971).

${ }^{5}$ J. A. Davis, V. K. De, and J. D. Meindl, "A stochastic wire-length distribution for gigascale integration (GSI)—Part I: Derivation and validation," IEEE Trans. Electron Devices 45(3), 580 (1998).

${ }^{6}$ J. A. Davis, V. K. De, and J. D. Meindl, "A stochastic wire-length distribution for gigascale integration (GSI) - Part II: Applications to clock frequency, power dissipation, and chip size estimation," IEEE Trans. Electron Devices 45(3), 590 (1998).

${ }^{7}$ S. S. Iyer, "Orthogonal scaling to extend computing into the cognitive era," paper presented at Semicon Singapore, 2014.

${ }^{8}$ J. Van Olmen, A. Mercha, G. Katti, C. Huyghebaert, J. Van Aelst, E. Seppala, Z. Chao, S. Armini, J. Vaes, R. C. Teixeira, M. Van Cauwenberghe, P. Verdonck, K. Verhemeldonck, A. Jourdain, W. Ruythooren, M. de P. de ten Broeck, A. Opdebeeck, T. Chiarella, B. Parvais, I. Debusschere, T. Y. Hoffmann, B. De Wachter, W. Dehaene, M. Stucchi, M. Rakowski, P. Soussan, R. Cartuyvels, E. Beyne, S. Biesemans, and B. Swinnen, "3D stacked IC demonstration using a through silicon via first approach," IEEE Int. Electron Devices Meet., Tech. Dig. 2008, 603.

${ }^{9}$ G. Katti, S. W. Ho, L. Ding, K. F. Chang, and S. Bhattacharya, "A costeffective platform for heterogeneous 2.5D ICs," in Chip Scale Review Magazine, May-June 2014, p. 16-20.

${ }^{10}$ J. R. Cubillo, R. Weerasekera, and G. Katti, "Through-silicon interposer (TSI) co-design optimization for high performance systems," in IEEE Electronics Packaging Technology Conference (EPTC) (2012), p. 93.

${ }^{11}$ N. Sturcken, E. J. O’Sullivan, N. Wang, P. Herget, B. C. Webb, L. T. Romankiw, M. Petracca, R. Davies, R. E. Fontana, G. M. Decad, I. Kymissis, A. V. Peterchev, L. P. Carloni, W. J. Gallagher, and K. L. Shepard, "A 2.5D integrated voltage regulator using coupled-magneticcore inductors on silicon interposer," IEEE J. Solid-State Circuits 48(1), 244 (2013).

${ }^{12}$ L. Madden, E. Wu, N. Kim, B. Banijamali, K. Abugharbieh, S. Ramalingam, and X. Wu, "Advancing high performance heterogeneous integration through die stacking," in Proceedings of European Solid-State Device Research Conference (ESSDERC), 2012.

${ }^{13}$ M. M. Corbalan, A. Keval, T. Toms, D. Lisk, R. Radojcic, and M. Nowak, "Power and signal integrity challenges in 3D systems," in Proceedings of Design Automation Conference (2013), p. 161.

${ }^{14}$ See http://www.jedec.org/standards-documents/docs/jesd235 for HBM.

${ }^{15}$ See http://www.hybridmemorycube.org/ for HMC.

${ }^{16} \mathrm{D}$. A. Miller, "Device requirements for optical interconnects to silicon chips," Proc. IEEE 97(7), 1166 (2009).

${ }^{17}$ W. Topol, D. C. La Tulipe, Jr., L. Shi, D. J. Frank, K. Bernstein, S. E. Steen, A. Kumar, G. U. Singco, A. M. Young, K. W. Guarini, and M. Ieong, "Three-dimensional integrated circuits," IBM J. Res. Dev. 50(4/5), 491 (2006)

${ }^{18}$ B. Swinnen, W. Ruythooren, P. De Moor, L. Bogaerts, L. Carbonell, K. De Munck, B. Eyckens, S. Stoukatch, D. Sabuncuoglu Tezcan, Z. Tokei, J. Vaes, J. Van Aelst, and E. Beyne, "3D integration by $\mathrm{Cu}-\mathrm{Cu}$ thermo compression bonding of extremely thinned bulk-Si die containing $10 \mu \mathrm{m}$ pitch through-Si vias," Technical Digest of the International Electron Device Meeting 2006, 371.

${ }^{19}$ J. U. Knickerbocker et al., "2.5 D and 3D technology challenges and test vehicle demonstrations," in Proceedings of Electronic Components and Technology Conference (ECTC) (2012), p. 1068.

${ }^{20}$ E. J. Marinissen, "Challenges and emerging solutions in testing TSVbased 2 1/2D-and 3D-stacked ICs," in Proceedings of the Conference on 
Design, Automation and Test in Europe. EDA Consortium (2012), p. 1277.

${ }^{21}$ S. W. Yoon, D. W. Yang, J. H. Koo, M. Padmanathan, and F. Carson, "3D TSV processes and its assembly/packaging technology," in Proceedings of 3D System Integration, San Fransico, USA (2009), p. 1.

${ }^{22}$ J. H. Lau, "Evolution, challenge, and outlook of TSV, 3D IC integration and 3d silicon integration," in Processing of International Symposium on Advanced Packaging Materials, Xiamen, China (2011), p. 462.

${ }^{23}$ J. H. Lau, "Evolution and outlook of TSV and 3D IC/Si integration," in Proceedings of the 12th Electronics Packaging Technology Conference, Singapore, 8-10 December 2010, p. 560.

${ }^{24}$ M. Puech, J. M. Thevenoud, J. M. Gruffat, N. Launay, N. Arnal, and P. Godinat, "Fabrication of 3D Packaging TSV using DRIE," in Proceeding of Design, Test, Integration and Packaging of MEMS/MOEMS Symposium, Nice, USA (2008), p. 109.

${ }^{25}$ J. Bhardwaj, H. Ashraf, and A. McQuarrie, "Dry silicon etching for MEMS," in Proceeding of Symposium on Microstructure Micro Fabrication System Annual Meeting Electrochemical Society, Montreal, Canada (1997), p. 1.

${ }^{26}$ N. Ranganathan, K. Prasad, N. Balasubramanian, and K. L. Pey, “A study of thermo-mechanical stress and its impact on through-silicon vias," J. Micromech. Microeng. 18, 075018 (2008).

${ }^{27}$ B. Wu, A. Kumar, and S. Pamarthy, "High aspect ratio silicon etch: A review," J. Appl. Phys. 108, 051101 (2010).

${ }^{28}$ C. K. Kang, S. M. Lee, I. D. Jung, P. G. Jung, S. J. Hwang, and J. S. Ko, "The fabrication of patternable silicon nanotips using deep reactive ion etching," J. Micromech. Microeng. 18, 075007 (2008)

${ }^{29}$ L. Zhang, D. F. Lim, H. Y. Li, S. Gao, and C. S. Tan, "Through silicon via fabrication with low-k dielectric liner and its implications on parasitic capacitance and leakage current,” Jpn. J. Appl. Phys., Part 1 51, 04 DB03 (2012)

${ }^{30}$ C. Laviron, B. Dunne, V. Lapras, P. Galbiati, D. Henry, F. Toia, S. Moreau, R. Anciant, C. Brunet-Manquat, and N. Sillon, "Via first approach optimisation for through silicon via applications," in Proceedings of the 59th Electronic Components and Technology Conference, Dan Diego, CA, USA, 26-29 May 2009, p. 14.

${ }^{31}$ A. Summanwar, F. Neuilly, and T. Bourouina, "Elimination of notching phenomenon which occurs while performing deep silicon etching and stopping on an insulating layer," in Ph.D. Research in Microelectronics and Electronics (IEEE, Istanbul, Turkey, 2008), p. 129

${ }^{32}$ M. J. Walker, "Comparison of Bosch and cryogenic process for patterning high-aspect-ratio features in silicon," Proc. SPIE 4407, 89 (2001).

${ }^{33}$ M. Wasilik and A. P. Pisano, "Low-frequency process for silicon-on-insulator deep reactive ion etching," Proc. SPIE 4592, 462 (2001).

${ }^{34}$ K. Yonekura, M. Kiritani, S. Sakamori, T. Yokoi, N. Fujiwara, and H. Miyatake, "Effects of charge build-up of underlying layer by high aspect ratio etching," Jpn. J. Appl. Phys., Part 1 37, 2314 (1998).

${ }^{35}$ S. K. Praveen, H. W. Tsan, and R. Nagarajan, "Conformal low temperature dielectric deposition process below $200{ }^{\circ} \mathrm{C}$ for TSV application," in Proceedings of 12th Electronics Packaging Technology Conference, Singapore (2010), p. 27.

${ }^{36}$ D. Archard, K. Giles, A. Price, S. Burgess, and K. Buchanan, "Low temperature PECVD of dielectric films for TSV application," in Proceedings of 60th Electronics Components and Technology Conference, Las Vegas, NV, USA (2010), p. 764.

${ }^{37}$ K.-W. Lee, H. Wang, J.-C. Bea, M. Murugesan, Y. Sutou, T. Fukushima, T. Tanaka, J. Koike, and M. Koyanagi, "Barrier properies of CVD Mn oxide layer to Cu diffusion for 3-D TSV," IEEE Electron Device Lett. 35, 114 (2014)

${ }^{38}$ D. Jung, K.-J. Moon, B.-L. Park, G. Choi, H.-K. Kang, and C. Chung, "Properties of isolation liner and electrical characteristics of high aspect ratio TSV in 3D stacking technology," in Proceedings of Advanced Semiconductor Manufacturing Conference, Saratoga Springs, NY, USA (2012), p. 198.

${ }^{39}$ Y. Li, Y. Civale, Y. Oba, A. Cockburn, J. H. Park, E. Beyne, I. De Wolf, and K. Croes, "Impact of barrier integrity on liner reliability in 3D through silicon vias," in Proceedings of IEEE International Reliability Physics Symposium, Anaheim, CA, USA (2013), p. 5C5.1.

${ }^{40}$ R. Beica, P. Siblerud, C. Sharbono, and M. Bernt, "Advanced metallization for 3D integration," in Proceedings of 10th Electronics Packaging Technology Conference, Singapore (2008), p. 212.

${ }^{41}$ B. Kim, C. Sharbono, T. Ritzdorf, and D. Schmauch, "Factors affecting copper filling process within high aspect ratio deep vias for $3 \mathrm{D}$ chip stacking," in Proceedings of 56th Electronic Components and Technology Conference, San Diego, CA, USA (2006), p. 838.

${ }^{42}$ J. W. Choi, L. G. Ong, Y. Mao, H. B. Mohamad, J. Xie, C. L. Chow, W. L. Loh, B. L. Lau, L. H. H. Liew, G. K. Lau, R. Murthy, and E. S. K. Tan, "TSV $\mathrm{Cu}$ filling failure modes and mechanisms causing the failures," IEEE Trans. Compon., Packag., Manuf. 4, 581 (2014).

${ }^{43}$ D. Malta, C. Gregory, D. Temple, T. Knutson, C. Wang, T. Richardson, and Y. Zhang, "Integrated process for defect-free copper plating and chemical-mechanical polishing of through-silicon vias for 3D interconnects," in Proceedings of 60th Electronic Components and Technology Conference, Las Vegas, NV, USA (2010), p. 1769.

${ }^{44}$ H. Y. Li, E. Liao, X. F. Pang, H. Yu, X. X. Yu, and J. Y. Sun, "Fast electroplating TSV process development for the via-last approach," in Proceedings of 60th Electronic Components and Technology Conference, Las Vegas, NV, USA (2010), p. 777.

${ }^{45}$ J. C. Chen, P. J. Tzeng, S. C. Chen, C. Y. Wu, C. C. Chen, Y. C. Hsin, J. H. Lau, Y. F. Hsu, S. H. Shen, S. C. Liao, C. H. Ho, C. H. Lin, T. K. Ku, and M. J. Kao, "Impact of slurry in Cu CMP (chemical mechanical polishing) on $\mathrm{Cu}$ topography of through silicon vias (TSVs), re-distribution layers, and $\mathrm{Cu}$ exposure," in Proceedings of 61 st Electronic Components and Technology Conference, Orlando, Florida, USA (2011), p. 1389.

${ }^{46}$ M. R. Oliver, Chemical-Mechanical Planarization of Semiconductor Material (Springer, 2004), p. 26.

${ }^{47}$ S. Tominaga, T. Enomoto, D. Abe, D. Takeda, K. Tanaka, H. Kitada, and T. Ohba, "Hybrid e-CMP/CMP process for $\mathrm{Cu}$ dual damascene TSV interconnects by using non-contact electrode e-CMP pad," in Proceeding of International Conference on Planarization/CMP Technology, Fukuoka, Japan, 19-21 November 2009, pp. 467-472.

${ }^{48}$ K. F. Yang, T. J. Wu, W. C. Chiou, M. F. Chen, Y. C. Liu, F. W. Tsai, C. C. Hsieh, C. H. Chang, W. J. Wu, Y. H. Chen, T. Y. Chen, H. R. Wang, I. C. Lin, S. B. Jan, R. D. Wang, Y. J. Lu, Y. C. Shih, H. A. Teng, C. S. Tsai, M. N. Chang, K. Chen, S. P. Jeng, and C. H. Yu, "Yield and reliability of 3DIC technology for advanced $28 \mathrm{~nm}$ node and beyond," in Proceedings of VLSI Technology Symposium, Honolulu, HI, USA (2011), p. 140.

${ }^{49}$ S. W. Ho, L. Ding, S. H. Lim, S. A. Sek, M. Yu, and G. Q. Lo, "Polymerbased fine pitch $\mathrm{Cu}$ RDL to enable cost-effective re-routing for $2.5 \mathrm{D}$ interposer and 3D-IC," in Proceedings of 15th Electronics Packaging Technology Conference (2013), p. 435.

${ }^{50}$ K. Kumagai, Y. Yoneda, H. Izumino, H. Shimojo, M. Sunohara, T. Kurihara, M. Higashi, and Y. Mabuchi, "A silicon interposer BGA package with $\mathrm{Cu}$-filled TSV and multi-layer $\mathrm{Cu}$-plating interconnect," in Proceedings of 61 st Electronic Components and Technology Conference, Lake Buena Vista, FL, USA (2008), p. 571.

${ }^{51}$ B. Williams, D. Florence, H. Dalai, and K. Gunturu, "RDL manufacturing for flip chip packaging," in Proceedings of IEEE Workshop on Microelectronics and Electron Devices, Boise, ID, USA (2005), p. 28.

${ }^{52}$ H. Y. Li, M. Tang, L. H. Guo, and G. Q. Lo, "BCB (Benzocylcobutene) process integration for the RF passive device," in Proceedings of 8th Electronics Packaging Technology Conference, Singapore (2008), p. 40.

${ }^{53}$ H. Y. Li, X. F. Pang, L. G. Ong, H. B. Li, W. S. Lee, N. Khan, K. H. Teo, and S. Gao, "Fine pitch low temperature RDL damascene process development for TSV integration," in Proceedings of 13th Electronics Packaging Technology Conference, Singapore (2011), p. 108.

${ }^{54}$ W.-S. Liao, C. C. Chiang, W. M. Wu, and C. H. Fan, "A highperformance low-cost chip-on-wafer package with sub- $\mu$ m pitch $\mathrm{Cu}$ RDL," in IEEE Symposium on VLSI, 2014.

${ }^{55}$ L. A. Polka, H. Kalyanam, G. Hu, and S. Krishnamoorthy, "Package technology to address the memory bandwidth challenge for tera-scale computing," Intel Technol. J. 11, 197 (2007).

${ }^{56}$ J. U. Knickerbocker, P. S. Andry, L. P. Buchwalter, A. Deutsch, R. R. Horton, K. A. Jenkins, Y. H. Kwark, G. McVicker, C. S. Patel, R. J. Polastre, C. Schuster, A. Sharma, S. M. Sri-Jayantha, C. W. Surovic, C. K. Tsang, B. C. Webb, S. L. Wright, S. R. McKnight, E. J. Sprogis, and B. Dang, "Development of next-generation system-on-package (SOP) technology based on silicon carriers with fine-pitch interconnection," IBM J. Res. Dev. 49, 725 (2005).

${ }^{57}$ K. Sakuma, P. S. Andry, B. Dang, J. Maria, C. K. Tsang, C. Patel, S. L. Wright, B. Webb, E. Sprogis, S. K. Kang, R. Polastre, R. Horton, and J. U. Knickerbocker, "3D chip stacking technology with low-volume leadfree interconnections," in Proceedings of 57th Electronic Components and Technology Conference, Reno, NV, USA (2007), p. 627.

${ }^{58}$ H. Hübner, S. Penka, M. Eigner, W. Gruber, M. Nobis, G. Kristen, M. Schneegans, B. Barchmann, and S. Janka, "Micro contacts with sub-30 
$\mu \mathrm{m}$ pitch for 3D chip-on-chip integration," in Proceedings of MAM, Grenoble, March 2006.

${ }^{59}$ Y. Tomita, T. Morifuji, T. Ando, M. Tago, R. Kajiwara, Y. Nemoto, T. Fujii, Y. Kitayama, and K. Takahashi, "Advanced packaging technologies on 3D stacked LSI utilizing the micro interconnections and the layered microthin encapsulation," in Proceedings of 51 st Electronic Components and Technology Conference, Orlando, FL, USA (2001), p. 353.

${ }^{60}$ K. Takahashi, M. Umemoto, N. Tanaka, K. Tanida, Y. Nemoto, Y. Tomita, M. Tage, and M. Bonkohara, "Ultra-high-density interconnection technology of three-dimensional packaging," Microelectron. Reliab. 43, 1267 (2003)

${ }^{61}$ S. L. Wright, R. Polastre, H. Gan, L. P. Buchwalter, R. Horton, P. S. Andry, E. Sprogis, C. Patel, C. Tsang, J. Knickerbocker, J. R. Lloyd, A. Sharma, and M. S. Sti-Jayantha, "Characterization of micro-bump C4 interconnects for Si-carrier SOP applications," in Proceedings of 55th Electronic Components and Technology Conference, San Diego, CA, USA (2006), p. 633.

${ }^{62}$ D. S. Patterson, "Towards 2.5/3D packaging enablement through copper pillar technology," in Chip Scale Review Magazine, May/June 2012.

${ }^{63} \mathrm{~K}$. Tanida, M. Umemoto, N. Tanaka, Y. Tomita, and K. Takahashi, "Micro Cu bump interconnection on 3D chip stacking technology," Jpn. J. Appl. Phys., Part 1 43, 2264 (2004).

${ }^{64}$ H. Gan, S. L. Wright, P. Polastre, L. P. Buchwalter et al., "Pb-free microjoints $(50 \mu \mathrm{m}$ pitch) for the next generation micro-systems: The fabrication, assembly and characterization," in Proceedings of the 55th Electronic Components and Technology Conference, San Diego, CA, USA (2005), p. 1210.

${ }^{65}$ A. Huffman, J. Lannon, M. Lueck, C. Gregory, and D. Temple, "Fabrication and characterization of metal-to-metal interconnect structures for 3-D integration," J. Instrum. 4, P03006 (2009).

${ }^{66}$ A. Yu, J. H. Lau, S. W. Ho, A. Kumar1, W. Y. Hnin, D.-Q. Yu1, and M. C. Jong, "Study of $15 \mu \mathrm{m}$ pitch solder microbumps for 3D IC integration," in Proceedings of the 55th Electronic Components and Technology Conference, San Diego, CA, USA (2009), p. 6.

${ }^{67}$ Y. Ohara, A. Noriki, K. Sakuma, K.-W. Lee, M. Murugesan, J. Bea, F. Yamada, T. Fukushima, T. Tanaka, and M. Koyanagi, "10 $\mu \mathrm{m}$ fine pitch $\mathrm{Cu} / \mathrm{Sn}$ micro-bumps for 3-D super-chip stack," in Proceedings of $3 D$ System Integration, San Francisco, CA (2009), p. 1.

${ }^{68}$ P. Soussan, W. Zhang, M. Pantouvaki, T. Delande, and S. Armini, "Assembly of $20 \mu \mathrm{m}$ pitch $\mathrm{Cu} / \mathrm{Sn}$ micro-bumps using high speed capillary self-alignment," in Proceedings of 3rd IEEE International Workshop on Low Temperature Bonding for 3D Integration, Tokyo Japan (2012), p. 153.

${ }^{69}$ J. De Vos, A. Jourdain, M. A. Erismis, W. Zhang, K. De Munck, A. La Manna, D. S. Tezcan, and P. Soussan, "High density $20 \mu \mathrm{m}$ pitch CuSn microbump process for high-end 3D applications," in Proceedings of the 61 st Electronic Components and Technology Conference, Lake Buena Vista, FL, USA (2011), p. 27.

${ }^{70}$ S.-Y. Huang, T.-C. Chang, R.-S. Cheng, J.-Y. Chang, C.-W. Fan, C.-J. Zhan, J. H. Lau, T.-H. Chen, W.-C. Lo, and M.-J. Kao, "Failure mechanism of $20 \mu \mathrm{m}$ pitch microjoint within a chip stacking architecture," in Proceedings of the 61st Electronic Components and Technology Conference, Lake Buena Vista, FL, USA (2011), p. 886.

${ }^{71}$ H.-Y. You, Y.-S. Lee, S.-K. Lee, and J.-S. Kang, "Reliability of $20 \mu \mathrm{m}$ micro bump interconnects," in Proceedings of the 61st Electronic Components and Technology Conference, Lake Buena Vista, FL, USA (2011), p. 608.

${ }^{72}$ R. Labie, F. Dosseul1, T. Webers, C. Winters, V. Cherman, E. Beyne, and B. Vandevelde, "Outperformance of Cu pillar flip chip bumps in electromigration testing," in Proceedings of the 61st Electronic Components and Technology Conference (ECTC), Lake Buena Vista, FL, USA (2011), p. 312.

${ }^{73}$ S. Olson, K. Hummler, and B. Sapp, "Challenges in thin wafer handling and processing," in Proceedings of 24th Advanced Semiconductor Manufacturing Conference, Saratoga Springs, NY, USA (2013), p. 62.

${ }^{74}$ T. Uhrmann, T. Matthias, M. Wimplinger, and J. Burggraf, "Recent progress in wafer processing," in Proceedings of 2013 International 3D Systems Integration Conference, San Francisco, CA, 2-4 October 2013, pp. $1-8$.

${ }^{75} \mathrm{~S}$. W. Yoon, D. J. Na, and W. K. Chooi, “2.5D/3D TSV process development and assembly/packaging technology," in Proceedings of the 13th IEEE-Electronic Packaging Technology Conference (2011), p. 337.

${ }^{76}$ L. C. Wai, X. Zhang, T. C. Chai, V. R. Srinivas, D. Ho, D. Pinjala, E. P. P. Myo, M. C. Jong, S. Lim, J. Ong, S. Thew, K. Chen, and V. N. Shekar, "Assembly of large dies fine pitch Cu/low-k FCBGA package with through silicon via (TSV) interposer," in Proceedings of the 11th IEEEElectronic Packaging Technology Conference (2009), p. 44.

${ }^{77}$ L. Lin, T.-C. Yeh, J.-L. Wu, G. Lu, T.-F. Tsai, L. Chen, and A.-T. Xu, "Reliability characterization of chip-on-wafer-on-substrate (CoWoS) 3D IC integration technology," in Proceedings of the IEEE-Electronic Components and Technology Conference (2013), p. 366.

${ }^{78}$ D. Y. Chen, W. C. Chiou, M. F. Chen, T. D. Wang, K. M. Ching, H. J. Tu, W. J. Wu, C. L. Yu, K. F. Yang, H. B. Chang, M. H. Tseng, C. W. Hsiao, Y. J. Lu, H. P. Hu, Y. C. Lin, C. S. Hsu, W. S. Shue, and C. H. Yu, "Enabling 3D-IC foundry technologies for $28 \mathrm{~nm}$ node and beyond: Through-silicon-via integration with high throughput die-to-wafer stacking," IEEE Int. Electron Devices Meet. 2009, 1-4.

${ }^{79}$ W. C. Chiou, K. F. Yang, J. L. Yeh, S. H. Wang, Y. H. Liou, T. J. Wu, J. C. Lin, C. L. Huang, S. W. Lu, C. C. Hsieh, H. A. Teng, C. C. Chiu, H. B. Chang, T. S. Wei, Y. C. Lin, Y. H. Chen, H. J. Tu, H. D. Ko, T. H. Yu, J. P. Hung, P. H. Tsai, D. C. Yeh, W. C. Wu, A. J. Su, S. L. Chiu, S. Y. Hou, D. Y. Shih, K. H. Chen, S. P. Jeng, and C. H. Yu, "An ultra-thin interposer utilizing 3D TSV technology," Symp. VLSI Technol., Dig. Tech. Pap. 2012, 107.

${ }^{80}$ M. H. Chan, H. N. Huang, C. F. Chan, C. T. Lin, M. H. Yang, and J. Y. Lai, "A study on the 3DIC interconnection using thermal compression bond with non-conductive paste process," in Proceedings of the International Microsystems, Packaging, Assembly and Circuits Technology Conference (2012), p. 274.

${ }^{81}$ M. Chew, E. Wai, C. T. Heang, D. Ho, S. Lim, S. C. Chong, T. C. Chai, and V. S. Rao, "Underfill characterization for multi-layer 3D-SiP stacked chip package," in Proceedings of the 13th IEEE-Electronic Packaging Technology Conference (2011), p. 246.

${ }^{82}$ J. U. Knickerbocker, P. S. Andry, E. Colgan, B. Dang, T. Dickson, X. Gu, C. Haymes, C. Jahnes, Y. Liu, J. Maria, R. J. Polastre, C. K. Tsang, L. Turlapati, B. C. Webb, L. Wiggins, and S. L. Wright, "2.5D and 3D technology challenges and test vehicle demonstrations," in Proceedings of the IEEE-Electronic Components \& Technology Conference (2012), p. 1068.

${ }^{83}$ C.-L. Su, K. Y. Yeh, and C. C. Lin, "Development of a non-conductive, no-flow wafer level underfill," in Proceedings of the IEEE-Electronic Components \& Technology Conference (2011), p. 1900.

${ }^{84}$ K. Murayama, M. Aizawa, K. Hara, M. Sunohara, K. Miyairi, K. Mori, J. Charbonnier, M. Assous, J.-P. Bally, G. Simon, and M. Higashi, "Warpage control of silicon interposer for 2.5D package application," in Proceedings of the IEEE-Electronic Components \& Technology Conference (ECTC) (2013), p. 879.

${ }^{85}$ G. Hariharan, R. Chaware, L. Yip, I. Singh, K. Ng, S. Y. Pai, M. Kim, H. Liu, and S. Ramalingam, "Assembly process qualification and reliability evaluations for heterogeneous 2.5D FPGA with HiCTE ceramic," in Proceedings of the IEEE-Electronic Components \& Technology Conference (2013), p. 904.

${ }^{86}$ R. Chaware, K. Nagarajan, and S. Ramalingam, "Assembly and reliability challenges in 3D integration of $28 \mathrm{~nm}$ FPGA die on a large high density $65 \mathrm{~nm}$ passive interposer," in Proceedings of the IEEE-Electronic Components \& Technology Conference (2012), p. 279.

${ }^{87}$ Y. Lu, W. Yin, B. Zhang, D. Yu, L. Wan, D. Shangguan, G. Xia, F. Qin, M. Ru, and F. Xiao, "A new 2.5D TSV package assembly approach," in Proceedings of the IEEE-Electronic Components \& Technology Conference (2013), p. 1965.

${ }^{88}$ A. Horibe, S. Kohara, K. Sueoka, K. Matsumoto, Y. Orii, and F. Yamada, "Effect of underfill properties on thermomechanical stress in fine pitch 3DIC package," in Proceedings of ICEP-IAAC, Tokyo, Japan (2012), p. 11.

${ }^{89}$ T. Hisada, Y. Yamada, J. Asai, and T. Aoki, "Study of warpage and mechanical stress of 2.5D package interposers during chip and interposer mount process," in Proceedings of IMAPS2012, San Diego, CA, USA (2012), p. 969.

${ }^{90}$ M. W. Le, J. Y. Kim, J. D. Kim, and C. H. Lee, "Below 45 nm low-k layer stress minimization guide for high-performance flip-chip packages with copper pillar bumping," in Proceedings of the IEEE-Electronic Components \& Technology Conference (2010), p. 1923.

${ }^{91}$ G. Cibrario, D. Henry, C. Chantre et al., "A 3D process kit generator based on customizable 3D layout design environment," in IEEE $3 D$ Systems Integration Conference (2013), p. 1.

${ }^{92}$ M. Stucchi, D. Perry, G. Katti, W. Dehaene, and D. Velenis, "Test structures for characterization of through silicon vias," IEEE Trans. Semicond. Manuf. 25(3), 355 (2012).

${ }^{93} \mathrm{~A}$. Bansal, "An analytical fringe capacitance model for interconnects using conformal mapping," IEEE Trans. Comput.-Aided Des. Integr. Circuits Syst. 25(12), 2765 (2006). 
${ }^{94}$ D. Sylvester, J. C. Chen, and C. Hu, "Investigation of interconnect capacitance characterization using charge-based-capacitance measurement (CBCM) technique and three-dimensional simulation," IEEE J. SolidState Circuits 33(3), 449 (1998).

${ }^{95}$ G. Katti, M. Stucchi, K. De Meyer, and W. Dehaene, "Electrical modeling and characterization of through silicon via for three-dimensional ICs," IEEE Trans. Electron Devices 57(1), 256 (2010).

${ }^{96}$ J. M. Rabaey, A. Chandrakasna, and B. Nikolic, Digital Integrated Circuit: A Design Perspective, 2nd ed. (Prentice-Hall, New Jersey, 2003).

${ }^{97}$ H. Kim, J. Cho, M. Kim et al., "Measurement and analysis of a highspeed TSV channel," IEEE Trans. Compon., Packag., Manuf. Technol. 2(10), 1672 (2012)

${ }^{98}$ L. Madden, "Heterogeneous 3-D stacking, can we have the best of both (technology) worlds," in 3D Architectures for Semiconductor Integration and Packaging (2012).

${ }^{99}$ G. Huang, M. S. Bakir, A. Naeemi, and J. D. Meindl, "Power delivery for 3-D chip stacks: Physical modeling and design implication," IEEE Trans. Compon., Packag., Manuf. Technol. 2(5), 852 (2012).

${ }^{100}$ V. Sridharn, M. Swaminatha, and T. Bandyopadhyay, "Enhancing signal and power integrity using double sided silicon interposer," IEEE Microwave Wireless Compon. Lett. 21(11), 598-600 (2011).

${ }^{101}$ L. Zhe, S. Hong, J. Xie, and A. Rahman, "Development of an optimized power delivery system for 3D IC integration with TSV silicon interposer," in Proceedings of IEEE Electronic Components and Technology Conference (ECTC) (2012), pp. 678-682.

${ }^{102}$ Yole Development, 3D Silicon \& Glass Interposers, August 2012.

${ }^{103}$ J. Lau, TSV Interposer: The Most Cost-Effective Integrator for 3D IC Integration, ASME Interpack 2011-52189, 2011.

${ }^{104}$ See http://www.inemi.org/2013-roadmap for iNEMI Technology Roadmap 2013.

${ }^{105}$ N. Khan, S. W. Yoon, A. G. K. Viswanath, V. P. Ganesh, R. Nagarajan, D. Witarsa, S. Lim, and K. Vaidyanathan, "Development of 3D stack package using silicon interposer for high power application," IEEE Trans. Adv. Packag. 31(1), 44 (2008).

${ }^{106}$ X. Y. Chen, K. C. Toh, T. N. Wong, J. C. Chai, D. Pinjala, and O. K. Navas, "Direct liquid cooling of a stacked MCM," in 8th IEEE ITHERM (2004)

${ }^{107}$ S. P. Tan, K. C. Toh, N. Khan, D. Pinjala, and V. Kripesh, "Development of single phase liquid cooling solution for 3-D silicon modules," IEEE Trans. Compon., Packag., Manuf. Technol. 1(4), 536 (2011).

${ }^{108}$ N. Khan, H. Y. Li, S. P. Tan, S. W. Ho, V. Kripesh, and D. Pinjala, "3-D packaging with through-silicon via (TSV) for electrical and fluidic interconnections," IEEE Trans. Compon., Packag., Manuf. Technol. 3(2), 221 (2013)

${ }^{109}$ G. Hoe, G. Y. Tang, D. Pinjala, C. T. Chong, J. H. Lau, X. W. Zhang et al., "Effect of TSV interposer on the thermal performance of FCBGA package," in 11th IEEE Electronics Packaging Technology Conference (EPTC) (2009).

${ }^{110}$ J. H. Lau and G. Y. Tang, "Effects of TSVs (through silicon vias) on thermal performances of3D IC integration system in package (SiP)," Microelectron. Reliab. 52(11), 2660 (2012).

${ }^{111}$ X. Q. Xing, Y. J. Lee, T. Y. Tee, X. W. Zhang, S. Gao, and W. S. Kwon, "Thermal modeling and characterization of package with through-siliconvia (TSV) interposer," in 13th IEEE Electronics Packaging Technology Conference (EPTC) (2011).

${ }^{112}$ H. Y. Zhang, X. W. Zhang, B. L. Lau, S. Lim, L. Ding, M. B. Yu, and Y. J. Lee, "Thermal characterization and simulation study of $2.5 \mathrm{D}$ packages with multi-chip module on through silicon interposer," in 15th IEEE Electronics Packaging Technology Conference (EPTC) (2013).

${ }^{113}$ K. Sikka, J. Wakil, H. Toy, and H. Liu, "An efficient lid design for cooling stacked flip-chip 3D packages," in 13th IEEE ITHERM (2012).

${ }^{114}$ H. Y. Zhang, D. Pinjala, T. N. Wong, and Y. K. Joshi, "Development of liquid cooling techniques for flip chip ball grid array packages with high flux heat dissipations," IEEE Trans. Compon. Packag. Technol. 28(1), 127 (2005).

${ }^{115}$ H. Y. Zhang, D. Pinjala, Y. K. Joshi, T. N. Wong, K. C. Toh, and M. K. Iyer, "Fluid Flow and Heat Transfer in Liquid Cooled Foam Heat Sinks for Electronic Packages," IEEE Trans. Compon. Packag. Technol. 28(2), $272(2005)$

${ }^{116}$ Y. Ye, J. Xu, X. Wu, W. Zhang, X. Wang, M. Nikdast, Z. Wang, and W. Liu, "System level modeling and analysis of thermal effects on optical networks-on-chip," IEEE Trans. Very Large Scale Integr. 21(2), 292 (2013).
${ }^{117}$ G. Zhang, Q. X. Zhang, C. T. Bui, G. Q. Lo, and B. Li, "Thermoelectric performance of silicon nanowires," Appl. Phys. Lett. 94(21), 213108 (2009).

${ }^{118}$ G. Zhang, Q. X. Zhang, K. Devi, and G. Q. Lo, “Time dependent thermoelectric performance of a bundle of $\mathrm{Si}$ nanowires for on chip cooler applications," Appl. Phys. Lett. 95(24), 243104 (2009).

${ }^{119}$ Y. Li, K. Buddharaju, N. Singh, and S. J. Lee, "Effect of electrical contact resistance in a silicon nanowire thermoelectric cooler and a design guideline for on-chip cooling applications," J. Electron. Mater. 42(7), 1476 (2013).

${ }^{120} \mathrm{~S}$. Hu, G. Hoe, H. Li, J. Shi, Y. Han, K. H. Teo, Y. Z. Xiong, J. He, and X. W. Zhang, "A thermal isolation technique using through-silicon vias for three-dimensional ICs," IEEE Trans. Electron Devices 60(3), 1282 (2013).

${ }^{121}$ JEDEC Standard JESD51-2A, Integrated Circuits Thermal Test Method Environmental Conditions-Natural Convection (Still Air), see http:// www.jedec.org/

${ }^{122}$ JEDEC Standard JESD 51-8, Integrated Circuits Thermal Test Method Environmental Conditions-Junction-to-Board, see http://www.jedec.org/

${ }^{123}$ C. S. Selvanayagam, J. H. Lau, X. W. Zhang, S. K. W. Seah, V. Kripesh, and T. C. Chai, "Nonlinear thermal stress/strain analyses of copper filled TSV (through silicon via) and their flip-chip microbumps," IEEE Trans. Adv. Packag. 32(4), 720 (2009).

${ }^{124}$ Y. S. Chan, H. Y. Li, and X. W. Zhang, "Thermo-mechanical design rules for the fabrication of TSV interposers," IEEE Trans. Compon., Packag., Manuf. Technol. 3(4), 633 (2013).

${ }^{125}$ F. X. Che, W. N. Putra, A. Heryanto, A. Trigg, X. W. Zhang, and C. L. Gan, "Study on $\mathrm{Cu}$ protrusion of through-silicon via," IEEE Trans. Compon., Packag., Manuf. Technol. 3(5), 732 (2013).

${ }^{126}$ C. H. Khong, T. C. Chai, S. W. Ho, and V. Kripesh, "Stress analysis of through silicon via (TSV) interposer using partial copper plated via with polymer filling," in Proceedings of 12th International Conference on Electronics Materials and Packaging, Singapore (2010), p. 80.

${ }^{127}$ C. S. Selvanayagam, X. W. Zhang, R. Rajoo, and D. Pinjala, "Modeling stress in silicon with TSVs and its effect on mobility," IEEE Trans. Compon., Packag., Manuf. Technol. 1(9), 1328 (2011).

${ }^{128}$ F. Che, H. Y. Li, X. W. Zhang, S. Gao, and K. H. Teo, "Development of wafer-level warpage and stress modeling methodology and its application in process optimization for TSV wafers," IEEE Trans. Compon., Packag., Manuf. Technol. 2(6), 944 (2012).

${ }^{129}$ F. X. Che, S. P. S. Lim, T. C. Chai, and X. W. Zhang, "Structure design optimization and reliability analysis on a pyramidal-shape three-diestacked package with through-silicon via," IEEE Trans. Device Mater. Reliab. 12(2), 201 (2012).

${ }^{130}$ X. W. Zhang, T. C. Chai, J. H. Lau, C. S. Selvanayagam, K. Biswas, and S. Liu, "Development of through silicon via (TSV) interposer technology for large die $(21 \times 21 \mathrm{~mm})$ fine-pitch $\mathrm{Cu} /$ low-k FCBGA package," in Proceedings of 59th Electronic Components and Technology Conference (EPTC), San Diego, CA, USA, May 2009, p. 305.

${ }^{131}$ F. X. Che, X. W. Zhang, N. Khan, K. H. Teo, S. Gao, and D. Pinjala, "The study of thermo-mechanical reliability for multi-layer stacked chip module with through-silicon-via (TSV)," in Proceedings of 12th Electronics Packaging Technology Conference, Singapore, December 2010, p. 743.

${ }^{132}$ X. W. Zhang, R. Rajoo, C. S. Selvanayagam, C. S. Premachandran, W. K. Choi, S. W. Ho, S. C. Ong, L. Xie, D. Pinjala, D.-L. Kwong, Y. M. Khoo, and S. Gao, "A low stress bond pad design for low temperature solder interconnections on through silicon vias (TSVs)," IEEE Trans. Compon., Packag., Manuf. Technol. 1(4), 510 (2011).

${ }^{133}$ X. W. Zhang, R. Rajoo, F. X. Che, C. S. Selvanayagam, W. K. Choi, S. Gao, G.-Q. Lo, and D.-L. Kwong, "A low stress bond pad design optimization of low temperature solder interconnections on TSVs for MEMS applications," in Proceedings of IEEE International 3D System Integration Conference 2011 (3DIC2011), Osaka, Japan, January 2012, pp. 1-5.

${ }^{134}$ D IC Compiler User Guide, Synopsys, Inc.

${ }^{135}$ D SOC Encounter User Guide, Cadence, Inc.

${ }^{136}$ G. Katti, M. Stucchi, D. Velenis, B. Soree, K. De Meyer, and W. Dehaene, "Temperature dependent modeling and characterization of through silicon via (TSV) capacitance," IEEE Electron Device Lett. 32(4), 563 (2011)

${ }^{137}$ Virtuoso Spectre Circuit Simulator Reference, Cadence, Inc.

${ }^{138}$ R. Weerasekera, J. R. Cubillo, and G. Katti, "Analysis of signal integrity (SI) robustness in through silicon interposer (TSI) interconnects," in Electronic Packaging Technology Conference (2012). 
${ }^{139}$ E. Schmidt, in Techonomy Conference in Lake Tahoe, CA, 2010.

${ }^{140}$ J. F. Gantz, D. Reinsel, C. Chute, W. Schlichting, J. McArthur, S. Minton, I. Xheneti, A. Toncheva, and A. Manfrediz, The Diverse and Exploding Digital Universe, IDC White Paper, 2007.

${ }^{141}$ J.-H. Huang, "Connecting people with ideas," paper presented at GPU Technology Conference, Nvidia, 2013.

${ }^{142}$ J. Furse, "Process methodologies for temporary thin wafer handling solutions," in Chip Scale Review Magazine, 2013.

${ }^{143}$ X. Zhang, T. C. Chai, J. H. Lau, C. S. Selvanayagam, K. Biswas, S. Liu, D. Pinjala, G. Y. Tang, Y. Y. Ong, S. R. Vempati, E. Wai, H. Y. Li, E. B. Liao, N. Ranganathan, V. Kripesh, J. Sun, J. Doricko, and C. J. Vath, "Development of Through Silicon Via (TSV) Interposer Technology for Large Die $(21 \times 21 \mathrm{~mm})$ Fine-pitch Cu/low-k FCBGA Package," 59th Electronic Components and Technology Conference (ECTC), 2009, 305.

${ }^{144}$ B. Banijamali, S. Ramalingam, K. Nagarajan, and R. Chaware, "Advanced reliability study of TSV interposers and interconnects for the $28 \mathrm{~nm}$ technology FPGA," in Electronic Components and Technology Conference (2011), p. 285.

${ }^{145}$ M. Murugasen, H. Kino, H. Nohira, J. C. Bea, A. Horibe, F. Yamada, C. Miyazaki, H. Kobayashi, T. Fukushima, T. Tanaka, and M. Koyanagi, "Wafer thinning, bonding, and interconnects induced local strain/stress in 3D LSI with fin-pitch high-density micro-bumps and through Si vias," IEEE Int. Electron Devices Meet. 2010, 30.

${ }^{146}$ E. Bersch, C. Kim, K. Hummler, and B. Sapp, "Cu-Cu direct bonding for ultra-high density chip-to-chip interconnects," in Chip Scale Review Magazine, Jul/Aug 2013, p. 20.

${ }^{147}$ J. D. Reed, M. Lueck, C. Gregory, C. A. Huffman, J. M. Lannon, and D. Temple, "High density interconnect at $10 \mu \mathrm{m}$ pitch with mechanically keyed $\mathrm{Cu} / \mathrm{Sn}-\mathrm{Cu}$ and $\mathrm{Cu}-\mathrm{Cu}$ bonding for 3D integration," in Proceedings of 60th Electronic Components and Technology Conference (ECTC) (IEEE, 2011), p. 846.

${ }^{148}$ C. W. C. Lin, S. C. L. Chiang, and T. K. A. Yang, "Bumpless flip chip packages for cost performance driven device," in Proceedings of Electronic Component and Technology Conference (ECTC) (2003), p. 231.

${ }^{149}$ A. Shigetou, T. Itoh, K. Sawada, and T. Suga, "Bumpless interconnect of $6 \mu \mathrm{m}$ pitch $\mathrm{Cu}$ electrodes at room temperature," in Proceedings of
Electronic Component and Technology Conference (ECTC) (2008), p. 1405 .

${ }^{150}$ K. Tanida, M. Umemoto, Y. Tomita, M. Tago, Y. Nemoto, T. Ando, and K. Takahashi, "Ultra-high density 3D chip stacking technology," in Proceedings of Electronic Component and Technology Conference (2003), p. 1084.

${ }^{151}$ A. Shigetou, T. Itoh, K. Sawada, and T. Suga, "Bumpless interconnect of $6 \mu \mathrm{m}$ pitch $\mathrm{Cu}$ electrodes at room temperature," IEEE Trans. Adv. Packag. 31(3), 473 (2008).

${ }^{152}$ T. Sakai, T. Akamatsu, N. Imaizumi, T. Miyajima, and M. Mizukoshi, "Cu-Cu thermo-compression bonding using ultra precision cutting of $\mathrm{Cu}$ bumps for 3D-IC," in Proceedings of the IMAPS 2011 Device Packaging Conference (2011), p. 146.

${ }^{153}$ N. Kouda, Fundamentals of Metallurgy (AGNE Gijistu Center, 2003), p. 117.

${ }^{154}$ S. Takaya, M. Nagata, A. Sakai, T. Kariya, S. Uchiyama, H. Kobayashi, and H. Ikeda, "A 100GB/s wide I/O with 4096b TSVs through an active silicon interposer with in-place waveform capturing," in IEEE International Solid-State Circuits Conference Digest of Technical Papers (2013).

${ }^{155}$ N. Kim, D. Wu, D. Kim, A. Rahman, and P. Wu, "Interposer design optimization for high frequency signal transmission in passive and active interposer using through silicon via (TSV)," in Electronic Components and Technology Conference (ECTC) (2011).

${ }^{156}$ T. Tanaka, H. Kino, K. Kiyoyama, H. Ohno, and M. Koyanagi, "Development of 3D-stacked reconfigurable spin logic chip using via-last backside-via 3D integration technology," in IEEE International Interconnect Technology Conference (IITC) (2013).

${ }^{157}$ R. Weerasekera, H. Y. Li, L. W. Yi, H. Sanming, J. Shi, J. Minkyu, and K. H. Teo, "On the impact of through-silicon-via-induced stress on $65 \mathrm{~nm}$ CMOS devices," IEEE Electron Device Lett. 34(1), 18-20 (2013).

${ }^{158}$ Y. Yang, G. Katti, R. Labie, Y. Travaly, B. Verlinden, and I. De Wolf, "Electrical evaluation of 130-nm MOSFETs with TSV proximity in 3DSIC structure," in IEEE International Interconnect Technology Conference (IITC) (2013).

${ }^{159}$ See http://www.cadence.com/community/blogs/ii/archive/2011/01/31/siliconrealization-design-methodology-boosts-3d-ics-with-tsvs.aspx for 3D IC tools. 